\title{
Nucleophilic Substitution at the Guanidine Carbon Center via Guanidine Cyclic Diimide Activation
}

\author{
Taeyang An and Yan Lee* \\ Department of Chemistry, College of Natural Sciences, Seoul National University, Seoul 08826, \\ Republic of Korea
}

Supporting Information 
Table of Contents
A. General Considerations
B. Preparation of Substrates
C. Optimization of Reaction Conditions with Amine Nucleophiles Table S1 and Table S2

D. General Procedures for the Nucleophilic Substitution at the Guanidine Carbon Center of GCDIs with Amines

E. Distortion of the Guanidine Structure in GCDI 1a Figure S1

F. ${ }^{15} \mathrm{~N}$ Incorporation Studies Figure S2

G. Hammett Plot Studies Table S3

H. Natural Bond Orbital (NBO) Analysis Figure S3 and Table S4-S7

I. NMR Studies of Protonation of GCDI 1a Figure S4

J. Investigation of Reaction Intermediates Figure S5 and Figure S6

K. LC-MS Analysis of the Reaction with Alkylamine Figure $\mathbf{S} 7$ and Figure S8

L. A Proposed Mechanism for Formation of $4 a$ Figure 59

M. Optimization of Reaction Conditions with Alcohol Nucleophiles Table S8

N. General Procedures for the Nucleophilic Substitution at the Guanidine Carbon Center of GCDIs with Alcohols

O. Single Crystal X-Ray Diffraction (SC-XRD) Analysis of $4 \mathrm{~m}$ Figure S10 and Table S9

P. Synthesis of 2-Aminobenzimidazole 5

Q. Synthesis of 2-Aminobenzoxazole 6

R. Synthesis of Sigma1 Inhibitor 7 (CT-189)

S. Synthesis of $\alpha$-Glucosidase Inhibitor 8

T. NMR Spectra

U. References 


\section{A. General Considerations}

${ }^{15} \mathrm{~N}$-aniline was obtained from Cambridge Isotope Laboratories. Other commercially available reagents were obtained from Sigma-Aldrich, Alfa Aesar or $\mathrm{TCl}$ and used without further purification.

${ }^{1} \mathrm{H}$ and ${ }^{13} \mathrm{C}$ NMR spectra were recorded on a Varian NMR System $500 \mathrm{MHz}$ spectrometer $(500 \mathrm{MHz}$ for ${ }^{1} \mathrm{H}$ and $125 \mathrm{MHz}$ for ${ }^{13} \mathrm{C}$ ), an Agilent NMR system $400 \mathrm{MHz}$ DD2MR400 spectrometer $\left(400 \mathrm{MHz}\right.$ for ${ }^{1} \mathrm{H}$ and $100 \mathrm{MHz}$ for ${ }^{13} \mathrm{C}$ ) and a Bruker Avance DPX-300 spectrometer (300 MHz for ${ }^{1} \mathrm{H}$ and $75 \mathrm{MHz}$ for $\left.{ }^{13} \mathrm{C}\right) .{ }^{1} \mathrm{H}$ chemical shifts were referenced from the chemical shifts of residual solvent peaks $(7.26 \mathrm{ppm}$ for $\mathrm{CDCl}_{3}, 4.79 \mathrm{ppm}$ for $\mathrm{D}_{2} \mathrm{O}, 3.31 \mathrm{ppm}$ for $\mathrm{CD}_{3} \mathrm{OD}, 1.94 \mathrm{ppm}$ for $\mathrm{CD}_{3} \mathrm{CN}$ and $2.50 \mathrm{ppm}$ for $\mathrm{DMSO}-\mathrm{d}_{6}$ ). ${ }^{13} \mathrm{C}$ NMR spectra were recorded with complete proton decoupling. ${ }^{13} \mathrm{C}$ chemical shifts were referenced from the chemical shifts of $\mathrm{CDCl}_{3}(77.16 \mathrm{ppm}), \mathrm{CD}_{3} \mathrm{OD}$ (49.00 ppm), $\mathrm{CD}_{3} \mathrm{CN}$ (1.32 ppm) or DMSO-d (39.52 ppm). Recorded spectral data were processed with the MestReNova (Version 6.0.2). Chemical shifts were reported in parts per million and the following abbreviations (or combinations thereof) were used to explain multiplicities: $\mathrm{s}=$ singlet, $\mathrm{d}=$ doublet, $\mathrm{t}=$ triplet, $\mathrm{q}=$ quartet, $\mathrm{m}=$ multiplet, $\mathrm{brs}=$ broad singlet, app $s=$ apparent singlet. Coupling constants, $J$, were reported in Hertz unit $(\mathrm{Hz}) \cdot{ }^{1} \mathrm{H}-{ }^{15} \mathrm{~N}$ heteronuclear multiple bond correlation (HMBC) experiments were performed for compound $\mathbf{3}$ on a Bruker Avance III-500 spectrometer (500 MHz for ${ }^{1} \mathrm{H}$ and $50.7 \mathrm{MHz}$ for $\left.{ }^{15} \mathrm{~N}\right) .{ }^{15} \mathrm{~N}$ chemical shifts were referenced from the chemical shifts of neat, external nitromethane (380.5 ppm, coaxial capillary). Mass spectra (MS) were recorded on an Agilent 6120 Quadrupole LCMS System using ESI-TOF (electrospray ionization-time of flight) mass spectrometer. High resolution mass spectroscopy (HRMS) analyses were performed at the Mass Spectrometry Laboratory of National Instrumentation Center for Environmental Management (NICEM) in Seoul National University. The MALDI-MS spectrum of $\mathbf{4 k}$ was recorded on a Bruker Daltonics Microflex LT using dithranol as a matrix and sodium trifluoroacetate as a cation source.

Analytical thin layer chromatography (TLC) was carried out on pre-coated glass silica gel plates with F254 indicator, and the spots were visualized under $254 \mathrm{~nm}$ UV irradiation and/or staining by $\mathrm{KMnO}_{4}$ solution or ninhydrin solution. Flash column chromatography was performed on silica gel (230-400 mesh) using an appropriate eluent system. 


\section{B. Preparation of Substrates}

\section{Preparation of guanidines}

Guanidines S1-S7 were prepared following the synthetic procedures reported in the previous literatures ${ }^{1,2}$.

General Procedure for the preparation of alkyl guanidines $(P 1)^{1}$

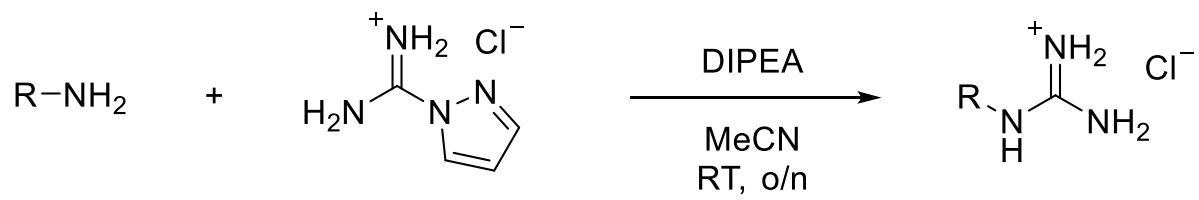

To a stirred solution of 1-amidinopyrazole hydrochloride and $N, N$-diisopropylethylamine (DIPEA) in acetonitrile $(\mathrm{MeCN})$, amine was added. The reaction mixture was stirred at ambient temperature for overnight, then concentrated under reduced pressure. The crude mixture was diluted with minimal amount of methanol $(\mathrm{MeOH})$, then excess amount of diethyl ether was added. The resulting precipitate was collected, further washed with diethyl ether, then dried in vacuo to afford guanidines as hydrochloride salts.

General Procedure for the preparation of aromatic guanidines $(P 2)^{2}$

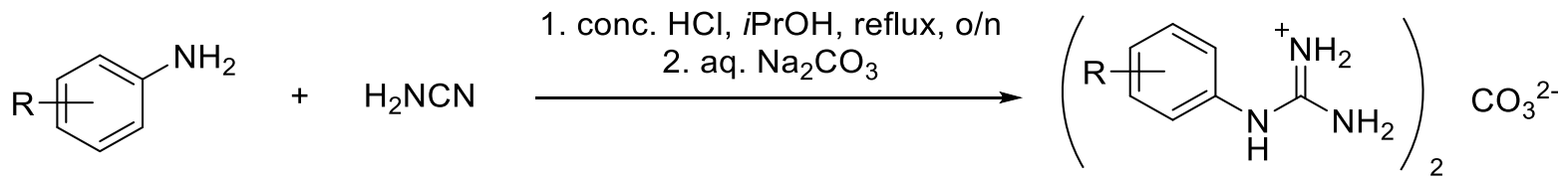

To a stirred solution of cyanamide and amine in isopropanol (iPrOH), concentrated hydrochloric acid was added. The reaction mixture was placed in an oil bath and stirred under reflux for overnight, then cooled to RT. Subsequently, saturated $\mathrm{Na}_{2} \mathrm{CO}_{3}$ solution was added, then the resulting precipitate was collected. The precipitate was further washed with water and acetone, then dried in vacuo to afford guanidines as carbonate salts.

Butylguanidine hydrochloride (S1)<smiles>CCCCNC(N)=[NH2+]</smiles>

S1

Following General Procedure P1, 1-amidinopyrazole hydrochloride (5.86 g, $40.0 \mathrm{mmol}, 1.00$ equiv.), DIPEA ( $7.14 \mathrm{ml}, 42.0 \mathrm{mmol}, 1.05$ equiv.), MeCN (30.0 $\mathrm{ml})$, and $n$-butylamine $(4.15 \mathrm{ml}, 42.0 \mathrm{mmol}, 1.05$ equiv.) yielded $\mathbf{S 1}(6.19 \mathrm{~g}$, $>99 \%)$ as a colorless gel. ${ }^{1} \mathrm{H}$ NMR $\left(500 \mathrm{MHz}, \mathrm{D}_{2} \mathrm{O}\right): \delta 3.17(\mathrm{t}, J=7.0 \mathrm{~Hz}, 2 \mathrm{H})$, $1.60-1.51(\mathrm{~m}, 2 \mathrm{H}), 1.41-1.30(\mathrm{~m}, 2 \mathrm{H}), 0.90(\mathrm{t}, J=7.4 \mathrm{~Hz}, 3 \mathrm{H})$. Spectral data match those previously reported ${ }^{1}$. 
Benzylguanidine hydrochloride (S2)<smiles>NC(=[NH2+])NCc1ccccc1</smiles>

S2

Following General Procedure P1, 1-amidinopyrazole hydrochloride (733 mg, $5.00 \mathrm{mmol}, 1.00$ equiv.), DIPEA (0.893 ml, $5.25 \mathrm{mmol}, 1.05$ equiv.), MeCN $(10.0 \mathrm{ml})$, and benzylamine $(0.573 \mathrm{ml}, 5.25 \mathrm{mmol}, 1.05$ equiv.) yielded $\mathbf{S 2}(760$ $\mathrm{mg}, 82 \%)$ as a white solid. ${ }^{1} \mathrm{H}$ NMR $\left(500 \mathrm{MHz}, \mathrm{D}_{2} \mathrm{O}\right): \delta 7.50-7.33(\mathrm{~m}, 5 \mathrm{H})$, $4.44(\mathrm{~s}, 2 \mathrm{H})$. Spectral data match those previously reported ${ }^{1}$.

Cyclohexylguanidine hydrochloride (S3)<smiles>NC(=[NH2+])NC1CCCCC1</smiles>

S3
Following General Procedure P1, 1-amidinopyrazole hydrochloride $(733 \mathrm{mg}$, $5.00 \mathrm{mmol}, 1.00$ equiv.), DIPEA (0.893 ml, $5.25 \mathrm{mmol}, 1.05$ equiv.), MeCN (10.0 $\mathrm{ml})$, and cyclohexylamine $(0.602 \mathrm{ml}, 5.25 \mathrm{mmol}, 1.05$ equiv.) yielded $\mathbf{S 3}(801 \mathrm{mg}$, $90 \%)$ as a white solid. ${ }^{1} \mathrm{H}$ NMR $\left(500 \mathrm{MHz}, \mathrm{D}_{2} \mathrm{O}\right): \delta 3.34-3.24(\mathrm{~m}, 1 \mathrm{H}), 1.92-1.83$ $(\mathrm{m}, 2 \mathrm{H}), 1.75-1.64(\mathrm{~m}, 2 \mathrm{H}), 1.60-1.52(\mathrm{~m}, 1 \mathrm{H}), 1.35-1.10(\mathrm{~m}, 5 \mathrm{H})$. Spectral data match those previously reported ${ }^{1}$.

Phenylguanidine carbonate (S4)<smiles>NC(=[NH2+])Nc1ccccc1</smiles>

S4

Following General Procedure P2, cyanamide $(841 \mathrm{mg}, 20.0 \mathrm{mmol}$, 2.00 equiv.), aniline ( $0.913 \mathrm{ml}, 10.0 \mathrm{mmol}, 1.00$ equiv.), iPrOH (10.0 $\mathrm{ml})$, concentrated hydrochloric acid $(1.00 \mathrm{ml})$ and saturated $\mathrm{Na}_{2} \mathrm{CO}_{3}$ solution $(20.0 \mathrm{ml})$ yielded $\mathbf{S 4}(628 \mathrm{mg}, 38 \%)$ as a white solid. ${ }^{1} \mathrm{H}$ NMR (500 MHz, DMSO- $\left.d_{6}\right): \delta 7.32-7.19(\mathrm{~m}, 2 \mathrm{H}), 7.05-6.83(\mathrm{~m}, 3 \mathrm{H}), 7.69-$ 3.61 (brs, $5 \mathrm{H}) .{ }^{13} \mathrm{C} \mathrm{NMR}\left(125 \mathrm{MHz}\right.$, DMSO- $\left.d_{6}\right): \delta 161.0,153.8,145.3$, 129.1, 123.2, 122.2. MS $(E S I)^{+}$calculated for $\mathrm{C}_{7} \mathrm{H}_{9} \mathrm{~N}_{3}[\mathrm{M}+\mathrm{H}]^{+}: \mathrm{m} / \mathrm{z}$

136.1, found 136.1 .

1-( $p$-Tolyl)guanidine carbonate (S5)<smiles>Cc1ccc(NC(N)=[NH2+])cc1</smiles>

S5
Following General Procedure P2, cyanamide (841 mg, 20.0 mmol, 2.00 equiv.), $p$-toluidine ( $1.07 \mathrm{~g}, 10.0 \mathrm{mmol}, 1.00$ equiv.), iPrOH $(10.0 \mathrm{ml})$, concentrated hydrochloric acid $(1.00 \mathrm{ml})$ and saturated $\mathrm{Na}_{2} \mathrm{CO}_{3}$ solution $(20.0 \mathrm{ml})$ yielded $\mathbf{S 5}(660 \mathrm{mg}, 37 \%)$ as a white solid. ${ }^{1} \mathrm{H}$ NMR $\left(500 \mathrm{MHz}\right.$, DMSO- $\left.d_{6}\right): \delta 7.02(\mathrm{~d}, J=$ $7.8 \mathrm{~Hz}, 2 \mathrm{H}), 6.75$ (d, J = 7.7 Hz, 2H), 6.86-4.45 (brs, 5H), 2.22 $(\mathrm{s}, 3 \mathrm{H})$. Spectral data match those previously reported ${ }^{2}$.

1-(4-Methoxyphenyl)guanidine carbonate (S6)

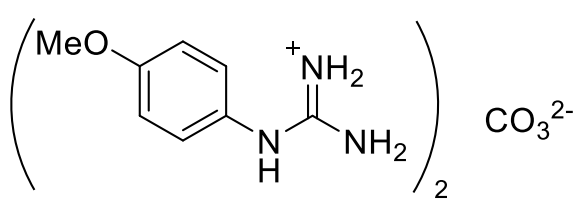

S6
Following General Procedure P2, cyanamide (841 mg, 20.0 mmol, 2.00 equiv.), $p$-anisidine ( $1.23 \mathrm{~g}, 10.0 \mathrm{mmol}, 1.00$ equiv.), $i \mathrm{PrOH}(10.0 \mathrm{ml})$, concentrated hydrochloric acid $(1.00 \mathrm{ml})$ and saturated $\mathrm{Na}_{2} \mathrm{CO}_{3}$ solution $(20.0 \mathrm{ml}$ ) yielded $\mathbf{S 6}(968 \mathrm{mg}, 49 \%)$ as a white solid. ${ }^{1} \mathrm{H}$ NMR $\left(400 \mathrm{MHz}\right.$, DMSO- $\left.d_{6}\right): \delta 6.90-6.73(\mathrm{~m}$, $4 \mathrm{H}), 7.00-4.52$ (brs, $5 \mathrm{H}), 3.70(\mathrm{~s}, 3 \mathrm{H}) .{ }^{13} \mathrm{C}$ NMR $(100 \mathrm{MHz}$, 
DMSO- $\left.d_{6}\right): \delta 154.9,154.0,138.2,124.6,123.9,114.4,55.2$. MS $(E S I)^{+}$calculated for $\mathrm{C}_{8} \mathrm{H}_{11} \mathrm{~N}_{3} \mathrm{O}[\mathrm{M}+\mathrm{H}]^{+}$: $\mathrm{m} / \mathrm{z} 166.1$, found 166.1 .

1-(4-Chlorophenyl)guanidine carbonate (S7)

$\left(\mathrm{H}_{\mathrm{H}}^{+\mathrm{NH}_{2}}\right)_{2}{ }_{\mathrm{CO}_{3}{ }^{2-}}$

S7

Following General Procedure P2, cyanamide $(841 \mathrm{mg}, 20.0$ mmol, 2.00 equiv.), 4-chloroaniline $(1.28 \mathrm{~g}, 10.0 \mathrm{mmol}, 1.00$ equiv.), $\mathrm{PrOH}(10.0 \mathrm{ml})$, concentrated hydrochloric acid $(1.00 \mathrm{ml})$ and saturated $\mathrm{Na}_{2} \mathrm{CO}_{3}$ solution $(20.0 \mathrm{ml})$ yielded $\mathbf{S} 7(1.19 \mathrm{~g}, 59 \%)$ as a white solid. ${ }^{1} \mathrm{H}$ NMR $\left(500 \mathrm{MHz}\right.$, DMSO- $\left.d_{6}\right): \delta 7.16(\mathrm{~d}, J=8.6$ $\mathrm{Hz}, 2 \mathrm{H}), 6.76$ (d, $J=8.4 \mathrm{~Hz}, 2 \mathrm{H}), 6.18-4.30$ (brs, $5 \mathrm{H}$ ). ${ }^{13} \mathrm{C}$ NMR (125 MHz, DMSO- $\left.d_{6}\right): \delta 152.7,150.0,128.6,124.6,123.5 . \mathrm{MS}$ $(E S I)^{+}$calculated for $\mathrm{C}_{7} \mathrm{H}_{8} \mathrm{CIN}_{3}[\mathrm{M}+\mathrm{H}]^{+}: \mathrm{m} / \mathrm{z}$ 170.1, found 170.1 .

The arginine derivative $\mathbf{S 8}$ was prepared following the synthetic procedures reported in the previous literatures ${ }^{1,3}$.

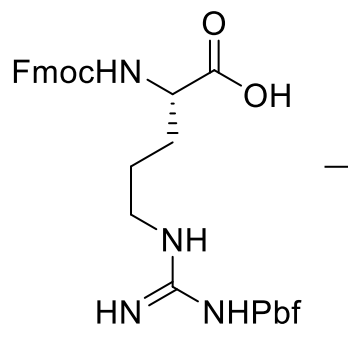

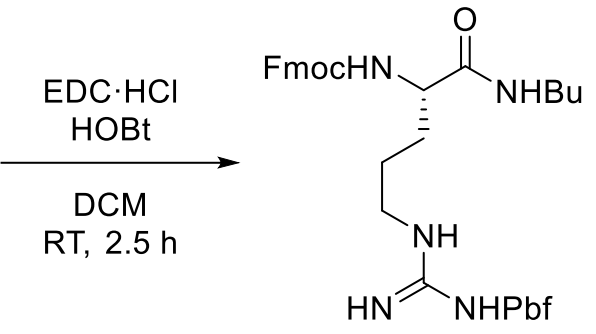

S9, $86 \%$

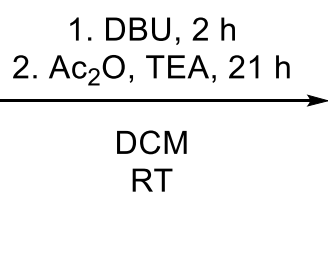<smiles>C=C(C)N[C@@H](CCCNC(=N)NCCCC)C(=O)NCCCC</smiles>

$\mathrm{S} 10,88 \%$
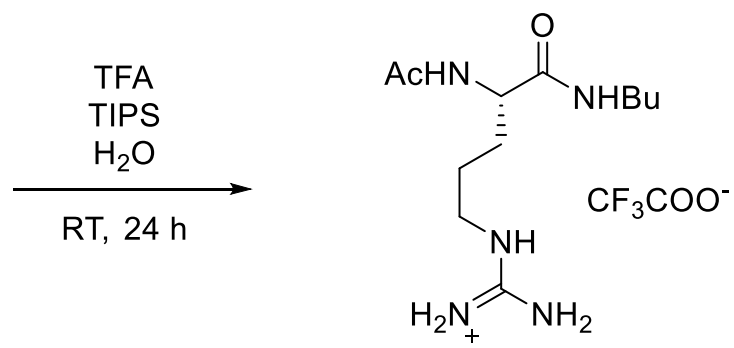

S8, $95 \%$ 
Fmoc-Arg(Pbf)-NH-( $n$-Bu) (S9)<smiles>CCCNC(=O)[C@H](CCCNC(=N)Nc1ccccc1)NC(F)F</smiles>

S9

To a stirred solution of Fmoc-Arg(Pbf)-OH (6.49 g, $10.0 \mathrm{mmol}, 1.00 \mathrm{eq}$.$) , 1-$ ethyl-3-(3-dimethylaminopropyl)carbodiimide hydrochloride (EDC $\cdot \mathrm{HCl})(2.11 \mathrm{~g}$, $11.0 \mathrm{mmol}, 1.10$ eq.) and hydroxybenzotriazole (HOBt) $(1.49 \mathrm{~g}, 11.0 \mathrm{mmol}$, 1.10 eq. $)$ in dichloromethane (DCM) $(300 \mathrm{ml})$ and $n$-butylamine $(1.09 \mathrm{ml}, 11.0$ mmol, 1.10 eq.) was added. The reaction mixture was stirred at ambient temperature for $2.5 \mathrm{~h}$, then concentrated under reduced pressure. The crude mixture was diluted with ethyl acetate and washed with a $0.01 \mathrm{M}$ aqueous $\mathrm{HCl}$ solution. The organic layer was further washed with brine. The combined organic layers were dried over anhydrous $\mathrm{MgSO}_{4}$, filtered, then concentrated under reduced pressure. $\mathbf{S 9}$ was afforded $(6.031 \mathrm{~g}, 86 \%)$ as a pale yellow solid after purification by flash column chromatography $\left(\mathrm{SiO}_{2}\right.$, hexane/ethyl acetate $10: 1 \rightarrow$ hexane/ethyl acetate 1:2). ${ }^{1} \mathrm{H}$ NMR $\left(300 \mathrm{MHz}, \mathrm{CDCl}_{3}\right): \delta 7.73(\mathrm{~d}, J=7.5 \mathrm{~Hz}, 2 \mathrm{H}), 7.55(\mathrm{~d}, J=7.5 \mathrm{~Hz}, 2 \mathrm{H}), 7.41-7.33$ (m, 2H), 7.30-7.21 (m, 2H), 7.02 (brs, 1H), 6.22 (brs, 2H), 6.12-5.87 (brs, 1H), $4.33(\mathrm{~d}, J=7.2 \mathrm{~Hz}, 2 \mathrm{H})$, 4.30-4.18 (m, 1H), 4.18-4.10 (m, 1H), 3.41-3.14 (m, 4H), $2.91(\mathrm{~s}, 2 \mathrm{H}), 2.58(\mathrm{~s}, 3 \mathrm{H}), 2.50(\mathrm{~s}, 3 \mathrm{H}), 2.07$ (s, 3H), 1.95-1.79 (m, 1H), 1.77-1.52 (m, 3H), 1.52-1.37 (m, 8H), 1.35-1.19 (m, 2H), $0.86(\mathrm{t}, J=7.3$ $\mathrm{Hz}, 3 \mathrm{H})$. Spectral data match those previously reported ${ }^{1}$.

$\operatorname{Ac}-\operatorname{Arg}(\mathrm{Pbf})-\mathrm{NH}-(n-\mathrm{Bu})(\mathbf{S 1 0})$<smiles>CCCCNC(=O)[C@H](CCCNC(=N)Nc1ccccc1)NC(C)=O</smiles>

S10

To a stirred solution of $\mathbf{S 9}(2.11 \mathrm{~g}, 3.00 \mathrm{mmol}, 1.00 \mathrm{eq}$.) in DCM $(30.0 \mathrm{ml}), 1,8-$ diazabicyclo[5.4.0]undec-7-ene (DBU) $(0.470 \mathrm{ml}, 3.15 \mathrm{mmol}, 1.05 \mathrm{eq}$.$) was added.$ The reaction mixture was stirred at ambient temperature for $2 \mathrm{~h}$. Acetic anhydride $(0.851 \mathrm{ml}, 9.00 \mathrm{mmol}, 3.00$ eq. $)$ and triethylamine (TEA) $(1.25 \mathrm{ml}, 9.00 \mathrm{mmol}, 3.00$ eq.) were added, then the reaction mixture was further stirred for $21 \mathrm{~h}$. Afterwards, the mixture was concentrated under reduced pressure, then diluted with ethyl acetate and washed with a saturated aqueous $\mathrm{NH}_{4} \mathrm{Cl}$ solution. The organic layer was further washed with a saturated $\mathrm{NaHCO}_{3}$ solution and brine. The combined organic layers were dried over anhydrous $\mathrm{MgSO}_{4}$, filtered, then concentrated under reduced pressure. $\mathbf{S 1 0}$ was afforded $(1.38 \mathrm{~g}, 88 \%)$ as a white solid after

purification by flash column chromatography $\left(\mathrm{SiO}_{2}, \mathrm{DCM} \rightarrow \mathrm{DCM} / \mathrm{MeOH}\right.$ 9:1). ${ }^{1} \mathrm{H} \mathrm{NMR}(500 \mathrm{MHz}$, $\left.\mathrm{CDCl}_{3}\right): \delta$ 7.40-7.32 (m, 2H), 6.39 (brs, 3H), 4.47-4.37 (m, 1H), 3.30-3.08 (m, 4H), $2.92(\mathrm{~s}, 2 \mathrm{H}), 2.53$ $(\mathrm{s}, 3 \mathrm{H}), 2.46(\mathrm{~s}, 3 \mathrm{H}), 2.05(\mathrm{~s}, 3 \mathrm{H}), 1.95(\mathrm{~s}, 3 \mathrm{H}), 1.84-1.72(\mathrm{~m}, 1 \mathrm{H}), 1.70-1.59(\mathrm{~m}, 1 \mathrm{H}), 1.59-1.45(\mathrm{~m}$, $2 \mathrm{H}), 1.45-1.34(\mathrm{~m}, 8 \mathrm{H}), 1.30-1.19(\mathrm{~m}, 2 \mathrm{H}), 0.82(\mathrm{t}, J=7.3 \mathrm{~Hz}, 3 \mathrm{H})$. Spectral data match those previously reported ${ }^{1}$.

Ac-Arg-NH-(n-Bu)·TFA (S8)<smiles>CCCCNC(=O)[C@H](CCCNC(N)=[NH2+])NC(C)=O</smiles>

S8
S10 (496 mg, $0.947 \mathrm{mmol}$ ) was dissolved in the mixed solution of trifluoroacetic acid (TFA), triisopropylsilane (TIPS) and $\mathrm{H}_{2} \mathrm{O}(2.85 \mathrm{ml} / 0.075$ $\mathrm{ml} / 0.075 \mathrm{ml}$ ). The reaction mixture was stirred at ambient temperature for $24 \mathrm{~h}$, then concentrated under reduced pressure. The crude mixture was diluted with $\mathrm{H}_{2} \mathrm{O}$ and washed with DCM. The aqueous layer was further washed with DCM and concentrated under reduced pressure. The arginine derivative S8 (346 mg, 95\%) was afforded as a pale yellow solid after drying in vacuo. ${ }^{1} \mathrm{H}$ NMR $\left(500 \mathrm{MHz}, \mathrm{D}_{2} \mathrm{O}\right): \delta 4.22-4.15(\mathrm{~m}, 1 \mathrm{H}), 3.27-3.11(\mathrm{~m}, 4 \mathrm{H})$, $2.01(\mathrm{~s}, 3 \mathrm{H}), 1.86-1.53(\mathrm{~m}, 4 \mathrm{H}), 1.51-1.40(\mathrm{~m}, 2 \mathrm{H}), 1.34-1.22(\mathrm{~m}, 2 \mathrm{H}), 0.86$ $(\mathrm{t}, J=7.3 \mathrm{~Hz}, 3 \mathrm{H})$. Spectral data match those previously reported ${ }^{1}$. 


\section{Preparation of GCDIs}

GCDIs 1a-1h were prepared following the synthetic procedures reported in our previous report ${ }^{1}$. The detailed procedures were modified as follows.

General Procedure P $3^{1}$

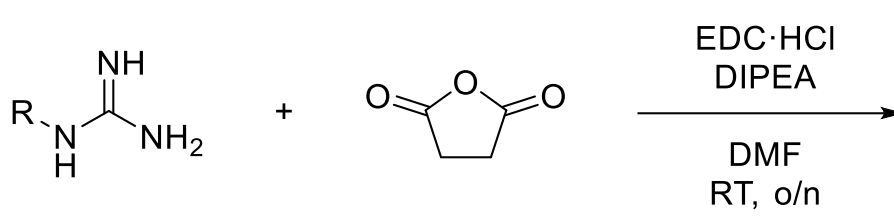<smiles>[R]N=C(N1C(=O)CCC1=O)N1C(=O)CCC1=O</smiles>

To a stirred solution of a guanidine salt, anhydride and EDC.HCl in dimethylformamide (DMF), DIPEA was added. The reaction mixture was stirred at ambient temperature for overnight, then concentrated under reduced pressure. The crude mixture was diluted with ethyl acetate and washed with $0.01 \mathrm{M}$ aqueous $\mathrm{HCl}$ solution. The organic layer was further washed with brine. The combined organic layers were dried over anhydrous $\mathrm{MgSO}_{4}$, filtered, then concentrated under reduced pressure. The product was purified by flash column chromatography.

1,1'-((Butylimino)methylene)bis(pyrrolidine-2,5-dione) (1a)<smiles>CCCCN=C(N1C(=O)CCC1=O)N1C(=O)CCC1=O</smiles>

1a

Following General Procedure P3, S1 (1.82 g, $12.0 \mathrm{mmol})$, succinic anhydride $(3.60 \mathrm{~g}, 36.0 \mathrm{mmol}), \mathrm{EDC} \cdot \mathrm{HCl}(4.60 \mathrm{~g}, 24.0 \mathrm{mmol}), \mathrm{DMF}(12.0 \mathrm{ml})$ and DIPEA $(10.2 \mathrm{ml}, 60.0 \mathrm{mmol})$ yielded GCDI 1a $(2.32 \mathrm{~g}, 69 \%)$ as a white solid after purification by flash column chromatography $\left(\mathrm{SiO}_{2}\right.$, hexane/ethyl acetate $5: 1$ $\rightarrow$ hexane/ethyl acetate 1:2). ${ }^{1} \mathrm{H} \mathrm{NMR}\left(500 \mathrm{MHz}, \mathrm{CDCl}_{3}\right): \delta 3.35(\mathrm{t}, J=7.0 \mathrm{~Hz}$, $2 \mathrm{H}), 2.80(\mathrm{~s}, 4 \mathrm{H}), 2.73(\mathrm{~s}, 4 \mathrm{H}), 1.66-1.56(\mathrm{~m}, 2 \mathrm{H}), 1.38-1.27(\mathrm{~m}, 2 \mathrm{H}), 0.84(\mathrm{t}$, $J=7.4 \mathrm{~Hz}, 3 \mathrm{H})$. Spectral data match those previously reported ${ }^{1}$.

1,1'-((Benzylimino)methylene)bis(pyrrolidine-2,5-dione) (1b)<smiles>O=C1CCC(=O)N1C(=NCc1ccccc1)N1C(=O)CCC1=O</smiles>

$1 b$

Following General Procedure P3, S2 $(1.11 \mathrm{~g}, 6.00 \mathrm{mmol})$, succinic anhydride $(1.80 \mathrm{~g}, 18.0 \mathrm{mmol}), \mathrm{EDC} \cdot \mathrm{HCl}(2.30 \mathrm{~g}, 12.0 \mathrm{mmol}), \mathrm{DMF}(6.00 \mathrm{ml})$ and DIPEA $(5.10 \mathrm{ml}, 30.0 \mathrm{mmol})$ yielded GCDI $1 \mathrm{~b}(1.39 \mathrm{~g}, 74 \%)$ as a white solid after purification by flash column chromatography $\left(\mathrm{SiO}_{2}\right.$, hexane/ethyl acetate $5: 1$ $\rightarrow$ hexane/ethyl acetate 1:2). ${ }^{1} \mathrm{H} \mathrm{NMR}\left(500 \mathrm{MHz}, \mathrm{CDCl}_{3}\right): \delta 7.39(\mathrm{~d}, J=7.6 \mathrm{~Hz}$, $2 \mathrm{H}), 7.36-7.31(\mathrm{~m}, 2 \mathrm{H}), 7.27-7.22(\mathrm{~m}, 1 \mathrm{H}), 4.73(\mathrm{~s}, 2 \mathrm{H}), 2.81(\mathrm{~s}, 4 \mathrm{H}), 2.79(\mathrm{~s}$, $4 \mathrm{H})$. Spectral data match those previously reported ${ }^{1}$. 
1,1'-((Cyclohexylimino)methylene)bis(pyrrolidine-2,5-dione) (1c)<smiles>O=C1CCC(=O)N1C(=NC1CCCCC1)N1C(=O)CCC1=O</smiles>

Following General Procedure P3, S3 $(712 \mathrm{mg}, 4.00 \mathrm{mmol})$, succinic anhydride $(1.20 \mathrm{~g}, 12.0 \mathrm{mmol}), \mathrm{EDC} \cdot \mathrm{HCl}(1.53 \mathrm{~g}, 8.00 \mathrm{mmol})$, DMF $(4.00 \mathrm{ml})$ and DIPEA (3.40 ml, $20.0 \mathrm{mmol})$ yielded GCDI 1c $(835 \mathrm{mg}, 68 \%)$ as a white solid after purification by flash column chromatography $\left(\mathrm{SiO}_{2}\right.$, hexane/ethyl acetate $5: 1 \rightarrow$ hexane/ethyl acetate 1:2). ${ }^{1} \mathrm{H} \mathrm{NMR}\left(500 \mathrm{MHz}, \mathrm{CDCl}_{3}\right): \delta 3.33-3.23(\mathrm{~m}, 1 \mathrm{H}), 2.83$ (s, 4H), $2.74(\mathrm{~s}, 4 \mathrm{H}), 1.83-1.72(\mathrm{~m}, 4 \mathrm{H}), 1.65-1.48(\mathrm{~m}, 3 \mathrm{H}), 1.32-1.19(\mathrm{~m}, 3 \mathrm{H})$. $1 \mathrm{c}$ Spectral data match those previously reported ${ }^{1}$.

1,1'-((Phenylimino)methylene)bis(pyrrolidine-2,5-dione) (1d)<smiles>O=C1CCC(=O)N1C(=Nc1ccccc1)N1C(=O)CCC1=O</smiles>

$1 d$

Following General Procedure P3, S4 (582 $\mathrm{mg}, 3.50 \mathrm{mmol})$, succinic anhydride $(1.06 \mathrm{~g}, 10.6 \mathrm{mmol}), \mathrm{EDC} \cdot \mathrm{HCl}(1.34 \mathrm{~g}, 7.00 \mathrm{mmol}), \mathrm{DMF}(3.50 \mathrm{ml})$ and DIPEA $(2.98 \mathrm{ml}, 17.5 \mathrm{mmol})$ yielded GCDI $1 \mathrm{~d}(739 \mathrm{mg}, 71 \%)$ as a white solid after purification by flash column chromatography $\left(\mathrm{SiO}_{2}\right.$, hexane/ethyl acetate $5: 1 \rightarrow$ hexane/ethyl acetate 1:3). ${ }^{1} \mathrm{H}$ NMR $\left(500 \mathrm{MHz}, \mathrm{CDCl}_{3}\right): \delta 7.33-7.27(\mathrm{~m}, 2 \mathrm{H})$, 7.17-7.10 (m, 1H), $6.92(\mathrm{~d}, J=7.5 \mathrm{~Hz}, 2 \mathrm{H}), 2.84(\mathrm{~s}, 4 \mathrm{H}), 2.64(\mathrm{~s}, 4 \mathrm{H}) .{ }^{13} \mathrm{C}$ NMR $\left(125 \mathrm{MHz}, \mathrm{CDCl}_{3}\right): \delta 173.5,173.0,144.7,129.6,129.0,125.9,119.9,28.4,28.3$. MS (ESI) ${ }^{+}$calculated for $\mathrm{C}_{15} \mathrm{H}_{13} \mathrm{~N}_{3} \mathrm{O}_{4}[\mathrm{M}+\mathrm{H}]^{+}: \mathrm{m} / \mathrm{z} 300.1$, found 300.0 .

1,1'-((p-Tolylimino)methylene)bis(pyrrolidine-2,5-dione) (1e)<smiles></smiles>

$1 e$

Following General Procedure P3, S5 $(631 \mathrm{mg}, 3.50 \mathrm{mmol})$, succinic anhydride $(1.06 \mathrm{~g}, 10.6 \mathrm{mmol}), \mathrm{EDC} \cdot \mathrm{HCl}(1.34 \mathrm{~g}, 7.00 \mathrm{mmol})$, DMF (3.50 $\mathrm{ml}$ ) and DIPEA (2.98 ml, $17.5 \mathrm{mmol})$ yielded GCDI 1e (932 mg, 85\%) as a white solid after purification by flash column chromatography $\left(\mathrm{SiO}_{2}\right.$, hexane/ethyl acetate $5: 1 \rightarrow$ hexane/ethyl acetate 1:3). ${ }^{1} \mathrm{H} \mathrm{NMR}(500 \mathrm{MHz}$, $\mathrm{CDCl}_{3}$ ): $\delta 7.09$ (d, J = 8.2 Hz, 2H), $6.82(\mathrm{~d}, J=8.2 \mathrm{~Hz}, 2 \mathrm{H}), 2.83(\mathrm{~s}, 4 \mathrm{H})$, $2.65(\mathrm{~s}, 4 \mathrm{H}), 2.29$ (s, 3H). ${ }^{13} \mathrm{C} \mathrm{NMR}\left(125 \mathrm{MHz}, \mathrm{CDCl}_{3}\right): \delta$ 173.5, 173.0, 142.2, 135.7, 129.7, 129.1, 119.9, 28.4, 28.3, 21.1. MS (ESI)+ calculated for $\mathrm{C}_{16} \mathrm{H}_{15} \mathrm{~N}_{3} \mathrm{O}_{4}[\mathrm{M}+\mathrm{H}]^{+}: \mathrm{m} / \mathrm{z} 314.1$, found 314.1.

1,1'-(((4-Methoxyphenyl)imino)methylene)bis(pyrrolidine-2,5-dione) (1f)<smiles>COc1ccc(N=C(N2C(=O)CCC2=O)N2C(=O)CCC2=O)cc1</smiles>

$1 f$
Following General Procedure P3, S6 (883 mg, $4.50 \mathrm{mmol})$, succinic anhydride $(1.35 \mathrm{~g}, 13.5 \mathrm{mmol}), \mathrm{EDC} \cdot \mathrm{HCl}(1.73 \mathrm{~g}, 9.00 \mathrm{mmol}), \mathrm{DMF}(4.50$ $\mathrm{ml})$ and DIPEA (3.83 $\mathrm{ml}, 22.5 \mathrm{mmol})$ yielded GCDI $1 \mathrm{f}(1.26 \mathrm{~g}, 85 \%)$ as a white solid after purification by flash column chromatography $\left(\mathrm{SiO}_{2}\right.$, hexane/ethyl acetate $5: 1 \rightarrow$ hexane/ethyl acetate $1: 3) .{ }^{1} \mathrm{H} \mathrm{NMR}(500 \mathrm{MHz}$, $\mathrm{CDCl}_{3}$ ): $\delta 6.89$ (d, $\left.J=8.9 \mathrm{~Hz}, 2 \mathrm{H}\right), 6.82(\mathrm{~d}, J=8.9 \mathrm{~Hz}, 2 \mathrm{H}), 3.77(\mathrm{~s}, 3 \mathrm{H})$, $2.83(\mathrm{~s}, 4 \mathrm{H}), 2.66(\mathrm{~s}, 4 \mathrm{H}) .{ }^{13} \mathrm{C}$ NMR $\left(125 \mathrm{MHz}, \mathrm{CDCl}_{3}\right): \delta$ 173.6, 173.0, 158.0, 137.9, 128.8, 121.6, 114.4, 55.5, 28.4, 28.3. MS (ESI)+ calculated for $\mathrm{C}_{16} \mathrm{H}_{17} \mathrm{~N}_{3} \mathrm{O}_{5}[\mathrm{M}+\mathrm{H}]^{+}: \mathrm{m} / \mathrm{z} 330.1$, found 330.0 . 
1,1'-(((4-Chlorophenyl)imino)methylene)bis(pyrrolidine-2,5-dione) (19)<smiles>O=C1CCC(=O)N1C(=Nc1ccc(Cl)cc1)N1C(=O)CCC1=O</smiles>

19

Following General Procedure P3, S7 (1.00 g, $5.00 \mathrm{mmol})$, succinic anhydride $(1.50 \mathrm{~g}, 15.0 \mathrm{mmol}), \mathrm{EDC} \cdot \mathrm{HCl}(1.92 \mathrm{~g}, 10.0 \mathrm{mmol}), \mathrm{DMF}(5.00$ $\mathrm{ml}$ ) and DIPEA (4.25 ml, $25.0 \mathrm{mmol})$ yielded GCDI $1 \mathrm{~g}(1.33 \mathrm{~g}, 80 \%)$ as a white solid after purification by flash column chromatography $\left(\mathrm{SiO}_{2}\right.$, hexane/ethyl acetate $5: 1 \rightarrow$ hexane/ethyl acetate 1:2). ${ }^{1} \mathrm{H} \mathrm{NMR}(500 \mathrm{MHz}$, $\left.\mathrm{CDCl}_{3}\right): \delta 7.25(\mathrm{~d}, J=8.6 \mathrm{~Hz}, 2 \mathrm{H}), 6.85(\mathrm{~d}, J=8.6 \mathrm{~Hz}, 2 \mathrm{H}), 2.83(\mathrm{~s}, 4 \mathrm{H})$, 2.66 (s, 4H). ${ }^{13} \mathrm{C} \mathrm{NMR}\left(125 \mathrm{MHz}, \mathrm{CDCl}_{3}\right): \delta 173.4,172.9,143.3,131.4$, 130.2, 129.2, 121.3, 28.4, 28.3. MS (ESI)+ calculated for $\mathrm{C}_{15} \mathrm{H}_{14} \mathrm{ClN}_{3} \mathrm{O}_{4}$

$[\mathrm{M}+\mathrm{H}]^{+}: \mathrm{m} / \mathrm{z} 334.1$, found 334.0 .

GCDI (1h)<smiles>CCCCNC(=O)[C@H](CCCN=C(N1C(=O)CCC1=O)N1C(=O)CCC1=O)NC(C)=O</smiles>

Following General Procedure P3, S8 (346 mg, $0.898 \mathrm{mmol}$ ), succinic anhydride (269 mg, $2.69 \mathrm{mmol}), \mathrm{EDC} \cdot \mathrm{HCl}(345 \mathrm{mg}, 1.80 \mathrm{mmol}), \mathrm{DMF}(0.900 \mathrm{ml})$ and DIPEA $(0.764 \mathrm{ml}, 4.49 \mathrm{mmol})$ yielded GCDI $1 \mathrm{~h}(121 \mathrm{mg}, 31 \%)$ as a white solid after purification by flash column chromatography $\left(\mathrm{SiO}_{2}\right.$, ethyl acetate $\rightarrow$ ethyl acetate/acetone 2:1). ${ }^{1} \mathrm{H} \mathrm{NMR}\left(500 \mathrm{MHz}, \mathrm{CDCl}_{3}\right): \delta 6.52-6.39(\mathrm{~m}, 2 \mathrm{H}), 4.13-4.02$ $(\mathrm{m}, 1 \mathrm{H}), 3.60-3.48(\mathrm{~m}, 2 \mathrm{H}), 3.30-3.11(\mathrm{~m}, 2 \mathrm{H}), 3.08-2.63(\mathrm{~m}, 8 \mathrm{H}), 2.06-1.96(\mathrm{~m}$, $2 \mathrm{H}), 1.92(\mathrm{~s}, 3 \mathrm{H}), 1.67-1.55(\mathrm{~m}, 2 \mathrm{H}), 1.52-1.43(\mathrm{~m}, 2 \mathrm{H}), 1.38-1.28(\mathrm{~m}, 2 \mathrm{H}), 0.89$ $(\mathrm{t}, J=7.3 \mathrm{~Hz}, 3 \mathrm{H})$. Spectral data match those previously reported ${ }^{1}$.

$1 \mathrm{~h}$

2,2'-((Butylimino)methylene)bis(isoindoline-1,3-dione) (1i)<smiles>CCCCN=C1C(=O)c2ccccc2C(=O)N1C(=O)c1ccccc1C</smiles>

$1 \mathbf{i}$
Following General Procedure P3, S1 (2.00 g, $13.2 \mathrm{mmol})$, phthalic anhydride (3.91 g, $26.4 \mathrm{mmol}), \mathrm{EDC} \cdot \mathrm{HCl}(5.06 \mathrm{~g}, 26.4 \mathrm{mmol}), \mathrm{DMF}(30.0$ $\mathrm{ml}$ ) and DIPEA (7.12 $\mathrm{ml}, 40.9 \mathrm{mmol})$ yielded GCDI $1 \mathrm{i}(2.18 \mathrm{~g}, 88 \%)$ as a white solid after purification by flash column chromatography $\left(\mathrm{SiO}_{2}\right.$, hexane/ethyl acetate $5: 1 \rightarrow$ hexane/ethyl acetate $2: 1) .{ }^{1} \mathrm{H} \mathrm{NMR}(300 \mathrm{MHz}$, $\mathrm{CDCl}_{3}$ ): $\delta 7.92(\mathrm{dd}, J=5.6,3.0 \mathrm{~Hz}, 2 \mathrm{H}), 7.87(\mathrm{dd}, J=5.6,3.0 \mathrm{~Hz}, 2 \mathrm{H}$ ), . $7.81(\mathrm{dd}, J=5.6,3.0 \mathrm{~Hz}, 2 \mathrm{H}), 7.76(\mathrm{dd}, J=5.5,3.2 \mathrm{~Hz}, 2 \mathrm{H}), 3.62(\mathrm{t}, J=$ $6.9 \mathrm{~Hz}, 2 \mathrm{H}), 1.85-1.71(\mathrm{~m}, 2 \mathrm{H}), 1.56-1.40(\mathrm{~m}, 2 \mathrm{H}), 0.94(\mathrm{t}, J=7.3 \mathrm{~Hz}$, $3 \mathrm{H})$. Spectral data match those previously reported ${ }^{1}$. 
(3aR,3a'R,7aS,7a'S)-2,2'-((Butylimino)methylene)bis(hexahydro-1H-isoindole-1,3(2H)-dione) (1j)

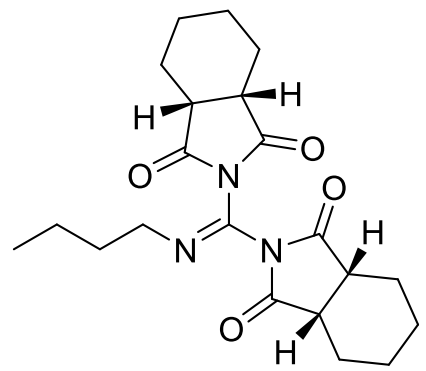

$1 \mathbf{j}$

Following General Procedure P3, S1 (303 mg, $2.00 \mathrm{mmol}$ ), ciscyclohexane-1,2-dicarboxylic anhydride $(925 \mathrm{mg}, 6.00 \mathrm{mmol}), \mathrm{EDC} \cdot \mathrm{HCl}$ (767 mg, $4.00 \mathrm{mmol})$, DMF $(4.00 \mathrm{ml})$ and DIPEA $(1.70 \mathrm{ml}, 10.0 \mathrm{mmol})$ yielded GCDI 1j (586 mg, 76\%) as a white solid after purification by flash column chromatography $\left(\mathrm{SiO}_{2}\right.$, hexane/ethyl acetate $5: 1 \rightarrow$ hexane/ethyl acetate 1:1). ${ }^{1} \mathrm{H}$ NMR $\left(400 \mathrm{MHz}, \mathrm{CDCl}_{3}\right): \delta 3.38(\mathrm{t}, J=6.8 \mathrm{~Hz}, 2 \mathrm{H}), 3.00-$ $2.92(\mathrm{~m}, 2 \mathrm{H}), 2.92-2.82(\mathrm{~m}, 2 \mathrm{H}), 1.91-1.72(\mathrm{~m}, 8 \mathrm{H}), 1.72-1.60(\mathrm{~m}, 2 \mathrm{H})$, $1.54-1.31(\mathrm{~m}, 10 \mathrm{H}), 0.88(\mathrm{t}, J=7.4 \mathrm{~Hz}, 3 \mathrm{H})$. Spectral data match those previously reported ${ }^{1}$. 


\section{Optimization of Reaction Conditions with Amine Nucleophiles}<smiles>CCCCN=C(N1C(=O)CCC1=O)N1C(=O)CCC1=O</smiles>

1a
1. Acid, $24 \mathrm{~h}$

2. aq. $\mathrm{NaOH}, 30 \mathrm{~min}$

Solvent (0.25 M)<smiles>CCCCN/C(N)=N/c1ccccc1</smiles>

$2 a$

To a flame-dried round bottom flask (RBF) equipped with a magnetic stirring bar, GCDI $1 \mathrm{a}(0.500 \mathrm{mmol}$, 1.00 eq.) was added, then the RBF was flushed with Ar. Solvent $(2.00 \mathrm{ml})$, acid, and aniline were added successively, then the reaction mixture was stirred at ambient temperature under $\mathrm{Ar}$ atmosphere. After $24 \mathrm{~h}$ reaction, the mixture was quenched by the addition of a $0.5 \mathrm{M}$ aqueous $\mathrm{NaOH}$ solution $(10.0 \mathrm{ml})$ and further stirred for $30 \mathrm{~min}$. The reaction mixture was concentrated under reduced pressure, diluted with ethyl acetate, then filtered. The yields were determined by ${ }^{1} \mathrm{H}$ NMR using 1,3,5-trimethoxybenzene as an internal standard.

Table S1. Representative Optimization Results of the Nucleophilic Substitution with Aniline

\begin{tabular}{|c|c|c|c|c|c|}
\hline Entry & Acid (eq.) & Solvent & Temperature & eq. of aniline & ${ }^{1} \mathrm{H}$ NMR Yields \\
\hline 1 & TFA (1.0) & $\mathrm{MeCN}$ & RT & 2.0 & $78 \%$ \\
\hline 2 & $\mathrm{MsOH}(1.0)$ & $\mathrm{MeCN}$ & RT & 2.0 & $32 \%$ \\
\hline 3 & $\mathrm{AlCl}_{3}(1.0)$ & $\mathrm{MeCN}$ & RT & 2.0 & $2 \%$ \\
\hline 4 & $\mathrm{AcOH}(1.0)$ & $\mathrm{MeCN}$ & RT & 2.0 & $28 \%$ \\
\hline 5 & TMSOTf (1.0) & $\mathrm{MeCN}$ & RT & 2.0 & $3 \%$ \\
\hline 6 & $\mathrm{MgCl}_{2}(1.0)$ & $\mathrm{MeCN}$ & RT & 2.0 & N.D. \\
\hline 7 & - & $\mathrm{MeCN}$ & RT & 2.0 & N.D. \\
\hline 8 & TFA (1.0) & DCM & RT & 2.0 & $76 \%$ \\
\hline 9 & TFA (1.0) & THF & RT & 2.0 & $70 \%$ \\
\hline 10 & TFA (1.0) & DMF & RT & 2.0 & $44 \%$ \\
\hline 11 & TFA (1.0) & Toluene & RT & 2.0 & $59 \%$ \\
\hline 12 & TFA (0.5) & $\mathrm{MeCN}$ & RT & 2.0 & $66 \%$ \\
\hline 13 & TFA (2.0) & $\mathrm{MeCN}$ & RT & 2.0 & $76 \%$ \\
\hline 14 & TFA (2.0) & $\mathrm{MeCN}$ & RT & 3.0 & $87 \%$ \\
\hline 15 & TFA (2.0) & MeCN & RT & 1.0 & $57 \%(49 \%)^{c}$ \\
\hline 16 & TFA (1.0) & $\mathrm{MeCN}$ & $70^{\circ} \mathrm{C}$ & 2.0 & $85 \%(82 \%)^{c}$ \\
\hline 17 & TFA (2.0) & MeCN & $70^{\circ} \mathrm{C}$ & 2.0 & $79 \%$ \\
\hline 18 & TFA (1.0) & $\mathrm{MeCN}$ & RT & 3.0 & $90 \%(84 \%)^{c}$ \\
\hline $19^{a}$ & TFA (1.0) & MeCN & RT & 3.0 & $80 \%$ \\
\hline $20^{b}$ & TFA (1.0) & $\mathrm{MeCN}$ & RT & 3.0 & $89 \%(88 \%)^{c}$ \\
\hline
\end{tabular}

a $3 \mathrm{~h}$ reaction. ${ }^{\mathrm{b}}$ Open-flask reaction. ${ }^{\mathrm{c}}$ Isolated yields.

Abbreviation: $\mathrm{MsOH}$ (methanesulfonic acid), $\mathrm{AcOH}$ (acetic acid) and TMSOTf (trimethylsilyl trifluoromethanesulfonate). 
Table S2. Reaction Yields for the Aniline Substitution on the GCDIs with Various Imide Groups ${ }^{a}$

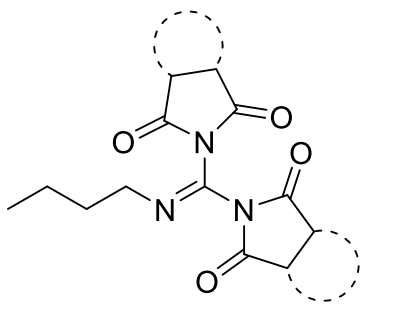

1<smiles>Nc1ccccc1</smiles>

3.0 eq.

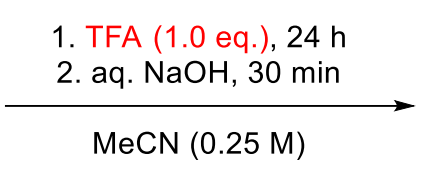

$\operatorname{MeCN}(0.25 \mathrm{M})$

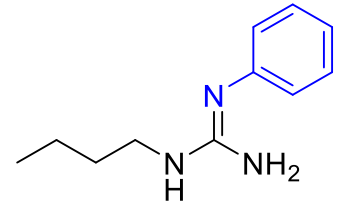

2a<smiles>CCCCN=C(N1C(=O)CCC1=O)N1C(=O)CCC1=O</smiles>

$1 \mathrm{a}, 89 \%$

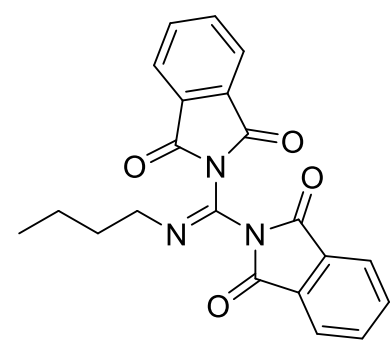

$1 i, 26 \%$

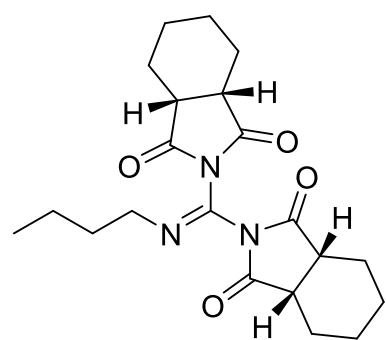

1j, $70 \%$

a ${ }^{1} \mathrm{H}$ NMR yields are given. 


\section{General Procedures for the Nucleophilic Substitution at the Guanidine Carbon Center of GCDls with Amines}

General Procedure A<smiles>[R1]N=C(N1C(=O)CCC1=O)N1C(=O)CCC1=O</smiles>

1<smiles>[R]c1ccc(N)cc1</smiles>

$3.0 \mathrm{eq}$.

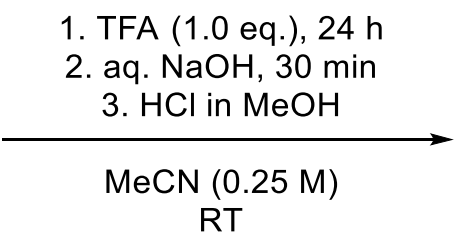

$\mathrm{RT}$<smiles>[R][X][NH+](c1ccccc1)C(N)N[R]</smiles>

2

To a stirred solution of GCDI 1 (0.500 mmol, 1.00 eq.) and TFA (0.0392 ml, $0.500 \mathrm{mmol}, 1.00$ eq.) in $\operatorname{MeCN}(2.00 \mathrm{ml})$, amine $(1.50 \mathrm{mmol}, 3.00$ eq.) was added, then the reaction mixture was stirred at ambient temperature. After $24 \mathrm{~h}$ reaction, the mixture was quenched by the addition of a $0.5 \mathrm{M}$ aqueous $\mathrm{NaOH}$ solution $(10.0 \mathrm{ml})$ and further stirred for $30 \mathrm{~min}$. The reaction mixture was concentrated under reduced pressure, diluted with ethyl acetate, then filtered. Subsequently, a $0.5 \mathrm{M} \mathrm{HCl}$ solution in $\mathrm{MeOH}$ $(15.0 \mathrm{ml})$ was added to the filtrate and the mixture was concentrated under reduced pressure. The residue was dissolved in DCM, then filtered. The product was purified by flash column chromatography.

\section{General Procedure B}<smiles>[R1]N=C(N1C(=O)CCC1=O)N1C(=O)CCC1=O</smiles>

1<smiles>[R]c1ccc(N)cc1</smiles>

3.0 eq.

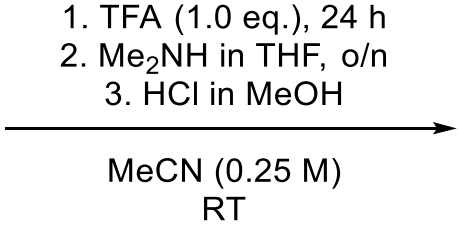<smiles>[R][X][NH+]=C(N)N[R]</smiles>

2

To a stirred solution of GCDI 1 (0.500 mmol, 1.00 eq.) and TFA (0.0392 ml, $0.500 \mathrm{mmol}, 1.00$ eq.) in MeCN $(2.00 \mathrm{ml})$, amine $(1.50 \mathrm{mmol}, 3.00$ eq.) was added, then the reaction mixture was stirred at ambient temperature. After $24 \mathrm{~h}$ reaction, the mixture was quenched by the addition of a $2.0 \mathrm{M}$ dimethylamine in tetrahydrofuran (THF) $(2.50 \mathrm{ml})$ and further stirred for overnight. The reaction mixture was concentrated under reduced pressure. Subsequently, a $0.5 \mathrm{M} \mathrm{HCl}$ solution in $\mathrm{MeOH}(15.0 \mathrm{ml})$ was added and the mixture was concentrated under reduced pressure. The residue was dissolved in DCM, then filtered. The product was purified by flash column chromatography.

1-Butyl-2-phenylguanidine hydrochloride (2a)<smiles>CCCCNC(N)=[NH+]c1ccccc1</smiles>

$2 a$
Guanidine 2a was afforded (100 mg, 88\%) following General Procedure A as a yellow gel after purification by flash column chromatography $\left(\mathrm{SiO}_{2}\right.$, $\mathrm{DCM} \rightarrow \mathrm{DCM} / \mathrm{MeOH}$ 9:1). ${ }^{1} \mathrm{H}$ NMR (500 MHz, $\left.\mathrm{CD}_{3} \mathrm{OD}\right): \delta 7.51-7.44(\mathrm{~m}$, $2 \mathrm{H}), 7.38-7.32(\mathrm{~m}, 1 \mathrm{H}), 7.29(\mathrm{~d}, J=7.7 \mathrm{~Hz}, 2 \mathrm{H}), 3.32(\mathrm{t}, J=6.8 \mathrm{~Hz}, 2 \mathrm{H})$, 1.69-1.59 (m, 2H), 1.50-1.40 (m, 2H), 0.99 (t, $J=7.4 \mathrm{~Hz}, 3 \mathrm{H}) .{ }^{13} \mathrm{C}$ NMR (125 MHz, CD $\left.{ }_{3} \mathrm{OD}\right): \delta 156.7,136.3,131.0,128.4,126.3,42.7,31.9,20.9$, 14.1. HRMS $(E S I)^{+}$calculated for $\mathrm{C}_{11} \mathrm{H}_{17} \mathrm{~N}_{3}[\mathrm{M}+\mathrm{H}]^{+}: \mathrm{m} / \mathrm{z}$ 192.1501, found 192.1490. 
1-Butyl-2-(4-methoxyphenyl)guanidine hydrochloride (2b)<smiles>CCCCNC(N)=[NH+]c1ccc(OC)cc1</smiles>

2b

Guanidine 2b was afforded (118 mg, 92\%) following General Procedure $A$ as a pale brown solid after purification by flash column chromatography $\left(\mathrm{SiO}_{2}, \mathrm{DCM} \rightarrow \mathrm{DCM} / \mathrm{MeOH}\right.$ 9:1). ${ }^{1} \mathrm{H}$ NMR $(500 \mathrm{MHz}$, $\mathrm{CD}_{3} \mathrm{OD}$ ): $\delta 7.21$ (d, $\left.J=8.9 \mathrm{~Hz}, 2 \mathrm{H}\right), 7.02(\mathrm{~d}, J=8.9 \mathrm{~Hz}, 2 \mathrm{H}), 3.82(\mathrm{~s}$, $3 \mathrm{H}), 3.28(\mathrm{t}, \mathrm{J}=7.1 \mathrm{~Hz}, 2 \mathrm{H}), 1.68-1.57(\mathrm{~m}, 2 \mathrm{H}), 1.49-1.38(\mathrm{~m}, 2 \mathrm{H})$, 0.98 (t, $J=7.4 \mathrm{~Hz}, 3 \mathrm{H}) .{ }^{13} \mathrm{C} \mathrm{NMR}\left(125 \mathrm{MHz}, \mathrm{CD}_{3} \mathrm{OD}\right): \delta 160.7,157.3$, 128.9, 128.3, 116.2, 56.1, 42.5, 32.0, 20.9, 14.1. MS (ESI) ${ }^{+}$calculated for $\mathrm{C}_{12} \mathrm{H}_{19} \mathrm{~N}_{3} \mathrm{O}[\mathrm{M}+\mathrm{H}]^{+}: \mathrm{m} / \mathrm{z} 222.2$, found 222.1.

1-Butyl-2-( $p$-tolyl)guanidine hydrochloride (2c)<smiles>CCCCNC(N)=[NH+]c1ccc(C)cc1</smiles>

2c

Guanidine 2c was afforded (113 mg, 93\%) following General Procedure $A$ as a pale yellow solid after purification by flash column chromatography $\left(\mathrm{SiO}_{2}, \mathrm{DCM} \rightarrow \mathrm{DCM} / \mathrm{MeOH} 9: 1\right) .{ }^{1} \mathrm{H} \mathrm{NMR}(500 \mathrm{MHz}$, $\mathrm{CD}_{3} \mathrm{OD}$ ): $\delta 7.28(\mathrm{~d}, J=7.5 \mathrm{~Hz}, 2 \mathrm{H}), 7.16(\mathrm{~d}, J=7.5 \mathrm{~Hz}, 2 \mathrm{H}), 3.30(\mathrm{t}, J=$ $6.2 \mathrm{~Hz}, 2 \mathrm{H}), 1.68-1.58(\mathrm{~m}, 2 \mathrm{H}), 1.50-1.39(\mathrm{~m}, 2 \mathrm{H}), 0.98(\mathrm{t}, J=7.2 \mathrm{~Hz}$, $3 \mathrm{H}) .{ }^{13} \mathrm{C}$ NMR $\left(125 \mathrm{MHz}, \mathrm{CD}_{3} \mathrm{OD}\right): \delta 156.9,138.7,133.5,131.5,126.7$, 42.6, 31.9, 21.1, 20.9, 14.1. MS $(\mathrm{ESI})^{+}$calculated for $\mathrm{C}_{12} \mathrm{H}_{19} \mathrm{~N}_{3}[\mathrm{M}+\mathrm{H}]^{+}$: $\mathrm{m} / \mathrm{z} 206.2$, found 206.1 .

1-Butyl-2-(4-(trifluoromethyl)phenyl)guanidine hydrochloride (2d)<smiles>CCCCNC(N)=[NH+]c1ccc(C(F)(F)F)cc1</smiles>

2d

Guanidine 2d was afforded (100 mg, 68\%) following General Procedure $A$ as a pale yellow gel after purification by flash column chromatography $\left(\mathrm{SiO}_{2}, \mathrm{DCM} \rightarrow \mathrm{DCM} / \mathrm{MeOH}\right.$ 9:1). ${ }^{1} \mathrm{H}$ NMR $\left(500 \mathrm{MHz}, \mathrm{CD}_{3} \mathrm{OD}\right): \delta 7.75$ (d, $J=8.4 \mathrm{~Hz}, 2 \mathrm{H}), 7.47(\mathrm{~d}, J=7.9 \mathrm{~Hz}, 2 \mathrm{H}), 3.34(\mathrm{t}, J=7.0 \mathrm{~Hz}, 2 \mathrm{H})$, $1.71-1.61(\mathrm{~m}, 2 \mathrm{H}), 1.50-1.40(\mathrm{~m}, 2 \mathrm{H}), 0.99(\mathrm{t}, J=7.4 \mathrm{~Hz}, 3 \mathrm{H}) .{ }^{13} \mathrm{C}$ NMR (125 MHz, $\left.\mathrm{CD}_{3} \mathrm{OD}\right): \delta 156.5,140.8,128.1,126.5,125.3,124.3,42.9$, $31.8,20.9,14.0 . \mathrm{MS}(\mathrm{ESI})^{+}$calculated for $\mathrm{C}_{12} \mathrm{H}_{16} \mathrm{~F}_{3} \mathrm{~N}_{3}[\mathrm{M}+\mathrm{H}]^{+}: \mathrm{m} / \mathrm{z} 260.1$, found 260.1 .

1-Butyl-2-(4-cyanophenyl)guanidine hydrochloride (2e)<smiles>CCCCNC(N)=[NH+]c1ccc(C#N)cc1</smiles>

$2 e$

Guanidine 2e was afforded (61 mg, 48\%) following General Procedure $A$ as a pale yellow solid after purification by flash column chromatography $\left(\mathrm{SiO}_{2}, \mathrm{DCM} \rightarrow \mathrm{DCM} / \mathrm{MeOH} 9: 1\right) .{ }^{1} \mathrm{H} \mathrm{NMR}(500 \mathrm{MHz}$, $\mathrm{CD}_{3} \mathrm{OD}$ ): $\delta 7.79(\mathrm{~d}, J=7.5 \mathrm{~Hz}, 2 \mathrm{H}), 7.43(\mathrm{~d}, J=7.8 \mathrm{~Hz}, 2 \mathrm{H}), 3.34(\mathrm{t}, J=$ $7.1 \mathrm{~Hz}, 2 \mathrm{H}), 1.71-1.61(\mathrm{~m}, 2 \mathrm{H}), 1.49-1.39(\mathrm{~m}, 2 \mathrm{H}), 0.99(\mathrm{t}, \mathrm{J}=7.2 \mathrm{~Hz}$, $3 \mathrm{H}) .{ }^{13} \mathrm{C}$ NMR (125 MHz, $\left.\mathrm{CD}_{3} \mathrm{OD}\right): \delta 156.2,142.2,135.0,124.7,119.3$, 110.0, 43.0, 31.7, 20.9, 14.0. MS (ESI)+ calculated for $\mathrm{C}_{12} \mathrm{H}_{16} \mathrm{~N}_{4}[\mathrm{M}+\mathrm{H}]^{+}$: $\mathrm{m} / \mathrm{z} 217.1$, found 217.1 . 
1-Butyl-2-(4-nitrophenyl)guanidine hydrochloride (2f)<smiles>CCCCNC(N)=[NH+]c1ccc([N+](=O)[O-])cc1</smiles>

$2 f$

Guanidine $\mathbf{2 f}$ was afforded (22 $\mathrm{mg}, 16 \%$ ) following General Procedure $A$ as a yellow gel after purification by flash column chromatography $\left(\mathrm{SiO}_{2}, \mathrm{DCM} \rightarrow \mathrm{DCM} / \mathrm{MeOH}\right.$ 9:1). ${ }^{1} \mathrm{H}$ NMR $\left(500 \mathrm{MHz}, \mathrm{CD}_{3} \mathrm{OD}\right): \delta 8.29$ (d, $J=9.0 \mathrm{~Hz}, 2 \mathrm{H}), 7.45(\mathrm{~d}, J=9.0 \mathrm{~Hz}, 2 \mathrm{H}), 3.34(\mathrm{t}, J=7.2 \mathrm{~Hz}, 2 \mathrm{H})$, 1.71-1.61 (m, 2H), 1.51-1.41 (m, 2H), $1.00(\mathrm{t}, J=7.4 \mathrm{~Hz}, 3 \mathrm{H}) .{ }^{13} \mathrm{C}$ NMR (125 MHz, $\mathrm{CD}_{3} \mathrm{OD}$ ): $\delta$ 156.3, 146.2, 144.2, 126.4, 124.1, 43.1, 31.7, 20.9, 14.0. MS (ESI)+ calculated for $\mathrm{C}_{11} \mathrm{H}_{16} \mathrm{~N}_{3} \mathrm{O}_{2}[\mathrm{M}+\mathrm{H}]^{+}: \mathrm{m} / \mathrm{z} 237.1$, found 237.0 .

1-Butyl-2-( $m$-tolyl)guanidine hydrochloride $(\mathbf{2 g})$<smiles>CCCCNC(N)=[NH+]c1cccc(C)c1</smiles>

$2 \mathrm{~g}$

Guanidine $\mathbf{2 g}$ was afforded (107 mg, 88\%) following General Procedure $A$ as a yellow gel after purification by flash column chromatography $\left(\mathrm{SiO}_{2}\right.$, $\mathrm{DCM} \rightarrow \mathrm{DCM} / \mathrm{MeOH}$ 9:1). ${ }^{1} \mathrm{H}$ NMR $\left(500 \mathrm{MHz}, \mathrm{CD}_{3} \mathrm{OD}\right): \delta$ 7.38-7.31 (m, $1 \mathrm{H}), 7.17(\mathrm{~d}, J=7.6 \mathrm{~Hz}, 1 \mathrm{H}), 7.10(\mathrm{~s}, 1 \mathrm{H}), 7.07(\mathrm{~d}, J=7.9 \mathrm{~Hz}, 1 \mathrm{H}), 3.30$ (t, $J=7.1 \mathrm{~Hz}, 2 \mathrm{H}), 2.38(\mathrm{~s}, 3 \mathrm{H}), 1.68-1.58(\mathrm{~m}, 2 \mathrm{H}), 1.50-1.39(\mathrm{~m}, 2 \mathrm{H})$, 0.99 (t, $J=7.4 \mathrm{~Hz}, 3 \mathrm{H}) .{ }^{13} \mathrm{C}$ NMR $\left(125 \mathrm{MHz}, \mathrm{CD}_{3} \mathrm{OD}\right): \delta 156.8,141.3$, $136.2,130.8,129.2,126.9,123.4,42.6,31.9,21.4,20.9,14.0 . \mathrm{MS}(\mathrm{ESI})^{+}$ calculated for $\mathrm{C}_{12} \mathrm{H}_{19} \mathrm{~N}_{3}[\mathrm{M}+\mathrm{H}]^{+}: \mathrm{m} / \mathrm{z}$ 206.2, found 206.2.

1-Butyl-2-(o-tolyl)guanidine hydrochloride (2h)<smiles>CCCCNC(N)=[NH+]c1ccccc1C</smiles>

$2 h$

Guanidine $\mathbf{2 h}$ was afforded (66 $\mathrm{mg}, 55 \%$ ) following General Procedure A as a purple gel after purification by flash column chromatography $\left(\mathrm{SiO}_{2}\right.$, $\mathrm{DCM} \rightarrow \mathrm{DCM} / \mathrm{MeOH}$ 9:1). ${ }^{1} \mathrm{H}$ NMR $\left(500 \mathrm{MHz}, \mathrm{CD}_{3} \mathrm{OD}\right): \delta$ 7.41-7.28 (m, $3 \mathrm{H}), 7.26-7.20(\mathrm{~m}, 1 \mathrm{H}), 3.30(\mathrm{t}, J=6.8 \mathrm{~Hz}, 2 \mathrm{H}), 2.30(\mathrm{~s}, 3 \mathrm{H}), 1.69-1.58(\mathrm{~m}$, $2 \mathrm{H}), 1.49-1.39(\mathrm{~m}, 2 \mathrm{H}), 0.99(\mathrm{t}, J=7.4 \mathrm{~Hz}, 3 \mathrm{H}) .{ }^{13} \mathrm{C}$ NMR $(125 \mathrm{MHz}$, $\left.\mathrm{CD}_{3} \mathrm{OD}\right): \delta 157.1,137.3,134.2,132.6,130.0,129.1,128.6,42.6,32.1,20.9$, 17.7, 14.0. MS $(\mathrm{ESI})^{+}$calculated for $\mathrm{C}_{12} \mathrm{H}_{19} \mathrm{~N}_{3}[\mathrm{M}+\mathrm{H}]^{+}: \mathrm{m} / \mathrm{z} 206.2$, found 206.2 .

1-Butyl-2-(4-fluorophenyl)guanidine hydrochloride (2i)<smiles>CCCCNC(N)=[NH+]c1ccc(F)cc1</smiles>

$\mathbf{2 i}$

Guanidine 2i was afforded (119 mg, 97\%) following General Procedure A as a pale brown gel after purification by flash column chromatography $\left(\mathrm{SiO}_{2}, \mathrm{DCM} \rightarrow \mathrm{DCM} / \mathrm{MeOH} 9: 1\right) .{ }^{1} \mathrm{H}$ NMR $\left(500 \mathrm{MHz}, \mathrm{CD}_{3} \mathrm{OD}\right): \delta 7.35-7.27$ $(\mathrm{m}, 2 \mathrm{H}), 7.24-7.18(\mathrm{~m}, 2 \mathrm{H}), 3.30(\mathrm{t}, J=6.9 \mathrm{~Hz}, 2 \mathrm{H}), 1.68-1.58(\mathrm{~m}, 2 \mathrm{H})$, 1.49-1.38 (m, 2H), $0.99(\mathrm{t}, J=7.4 \mathrm{~Hz}, 3 \mathrm{H}) .{ }^{13} \mathrm{C}$ NMR (125 MHz, CD $\left.{ }_{3} \mathrm{OD}\right)$ : $\delta$ 164.0, 162.1, 157.1, 132.4, 129.2, 117.8, 117.6, 42.7, 31.9, 20.9, 14.0. MS (ESI) ${ }^{+}$calculated for $\mathrm{C}_{11} \mathrm{H}_{16} \mathrm{FN} 3[\mathrm{M}+\mathrm{H}]^{+}: \mathrm{m} / \mathrm{z} 210.1$, found 210.1 . 
1-Butyl-2-(4-chlorophenyl)guanidine hydrochloride (2j)<smiles>CCCCNC(N)=[NH+]c1ccc(Cl)cc1</smiles>

2j

Guanidine 2j was afforded (113 mg, 86\%) following General Procedure A as a pale yellow gel after purification by flash column chromatography $\left(\mathrm{SiO}_{2}, \mathrm{DCM} \rightarrow \mathrm{DCM} / \mathrm{MeOH}\right.$ 9:1). ${ }^{1} \mathrm{H}$ NMR $\left(500 \mathrm{MHz}, \mathrm{CD}_{3} \mathrm{OD}\right): \delta 7.46$ (d, $J=8.1 \mathrm{~Hz}, 2 \mathrm{H}), 7.28(\mathrm{~d}, \mathrm{~J}=7.9 \mathrm{~Hz}, 2 \mathrm{H}), 3.31(\mathrm{t}, J=6.4 \mathrm{~Hz}, 2 \mathrm{H}), 1.68-$ $1.59(\mathrm{~m}, 2 \mathrm{H}), 1.50-1.39(\mathrm{~m}, 2 \mathrm{H}), 0.98(\mathrm{t}, J=7.3 \mathrm{~Hz}, 3 \mathrm{H}) .{ }^{13} \mathrm{C}$ NMR $(125$ $\left.\mathrm{MHz}, \mathrm{CD}_{3} \mathrm{OD}\right): \delta 156.7,135.3,133.7,131.0,127.9,42.7,31.8,20.9,14.0$. MS $(E S I)+$ calculated for $\mathrm{C}_{11} \mathrm{H}_{16} \mathrm{CIN}_{3}[\mathrm{M}+\mathrm{H}]^{+}$: $\mathrm{m} / \mathrm{z} 226.1$, found 226.1 .

1-Butyl-2-(4-bromophenyl)guanidine hydrochloride (2k)<smiles>CCCCNC(N)=[NH+]c1ccc(Br)cc1</smiles>

2k

Guanidine 2k was afforded (131 mg, 85\%) following General Procedure $A$ as a pale yellow solid after purification by flash column chromatography $\left(\mathrm{SiO}_{2}, \mathrm{DCM} \rightarrow \mathrm{DCM} / \mathrm{MeOH}\right.$ 9:1). ${ }^{1} \mathrm{H}$ NMR $\left(500 \mathrm{MHz}, \mathrm{CD}_{3} \mathrm{OD}\right): \delta 7.61$ (d, $J=7.4 \mathrm{~Hz}, 2 \mathrm{H}), 7.22(\mathrm{~d}, J=7.6 \mathrm{~Hz}, 2 \mathrm{H}), 3.30(\mathrm{t}, J=6.7 \mathrm{~Hz}, 2 \mathrm{H}), 1.69-$ $1.58(\mathrm{~m}, 2 \mathrm{H}), 1.51-1.39(\mathrm{~m}, 2 \mathrm{H}), 0.99(\mathrm{t}, \mathrm{J}=7.2 \mathrm{~Hz}, 3 \mathrm{H}) .{ }^{13} \mathrm{C}$ NMR $(125$ $\left.\mathrm{MHz}, \mathrm{CD}_{3} \mathrm{OD}\right): \delta$ 156.6, 135.8, 134.0, 128.1, 121.4, 42.8, 31.8, 20.9, 14.0. MS $(E S I)^{+}$calculated for $\mathrm{C}_{11} \mathrm{H}_{16} \mathrm{BrN}_{3}[\mathrm{M}+\mathrm{H}]^{+}: \mathrm{m} / \mathrm{z} 270.1272 .1$, found 270.0, 272.0 .

1-Butyl-2-(4-iodophenyl)guanidine hydrochloride (2I)<smiles>CCCCNC(N)=[NH+]c1ccc(I)cc1</smiles>

21

Guanidine 2l was afforded (109 mg, 62\%) following General Procedure A as a white solid after purification by flash column chromatography $\left(\mathrm{SiO}_{2}\right.$, $\mathrm{DCM} \rightarrow \mathrm{DCM} / \mathrm{MeOH}$ 9:1). ${ }^{1} \mathrm{H}$ NMR (500 MHz, $\left.\mathrm{CD}_{3} \mathrm{OD}\right): \delta 7.79$ (d, $J=8.6$ $\mathrm{Hz}, 2 \mathrm{H}), 7.07(\mathrm{~d}, J=8.5 \mathrm{~Hz}, 2 \mathrm{H}), 3.29(\mathrm{t}, J=7.1 \mathrm{~Hz}, 2 \mathrm{H}), 1.67-1.57(\mathrm{~m}$, $2 \mathrm{H}), 1.48-1.38(\mathrm{~m}, 2 \mathrm{H}), 0.98(\mathrm{t}, J=7.4 \mathrm{~Hz}, 3 \mathrm{H}) .{ }^{13} \mathrm{C} \mathrm{NMR}(125 \mathrm{MHz}$, $\left.\mathrm{CD}_{3} \mathrm{OD}\right): \delta 156.6,140.2,136.5,128.0,42.7,31.8,20.9,14.0 . \mathrm{MS}(\mathrm{ESI})^{+}$ calculated for $\mathrm{C}_{11} \mathrm{H}_{16} \mathrm{IN} \mathrm{N}_{3}[\mathrm{M}+\mathrm{H}]^{+}: \mathrm{m} / \mathrm{z} 318.0$, found 318.0 .

2-(4-Acetylphenyl)-1-butylguanidine hydrochloride (2m)<smiles>CCCCNC(N)=[NH+]c1ccc(C(C)=O)cc1</smiles>

$2 m$

Guanidine $2 \mathrm{~m}$ was afforded (107 mg, 79\%) following General Procedure $A$ as a yellow gel after purification by flash column chromatography $\left(\mathrm{SiO}_{2}, \mathrm{DCM} \rightarrow \mathrm{DCM} / \mathrm{MeOH} 9: 1\right) .{ }^{1} \mathrm{H}$ NMR $\left(500 \mathrm{MHz}, \mathrm{CD}{ }_{3} \mathrm{OD}\right): \delta 8.06$ (d, $J=8.5 \mathrm{~Hz}, 2 \mathrm{H}), 7.39(\mathrm{~d}, J=8.5 \mathrm{~Hz}, 2 \mathrm{H}), 3.35(\mathrm{t}, J=7.1 \mathrm{~Hz}, 2 \mathrm{H}), 2.60$ (s, 3H), 1.72-1.62 (m, 2H), 1.52-1.41 (m, 2H), $0.99(\mathrm{t}, J=7.4 \mathrm{~Hz}, 3 \mathrm{H})$. ${ }^{13} \mathrm{C}$ NMR $\left(125 \mathrm{MHz}, \mathrm{CD}_{3} \mathrm{OD}\right): \delta 199.2,156.3,141.8,135.8,131.3,124.2$, 43.0, 31.8, 26.7, 20.9, 14.0. MS (ESI)+ calculated for $\mathrm{C}_{13} \mathrm{H}_{19} \mathrm{~N}_{3} \mathrm{O}[\mathrm{M}+\mathrm{H}]^{+}$: $\mathrm{m} / \mathrm{z} 234.2$, found 234.1 . 
Ethyl 4-((amino(butylamino)methylene)amino)benzoate hydrochloride (2n)<smiles>CCCCNC(N)=[NH+]c1ccc(C(=O)OCC)cc1</smiles>

2n

Guanidine 2n was afforded (103 mg, 69\%) following General Procedure $B$ as a yellow solid after purification by flash column chromatography $\left(\mathrm{SiO}_{2}, \mathrm{DCM} \rightarrow \mathrm{DCM} / \mathrm{MeOH}\right.$ 9:1). ${ }^{1} \mathrm{H}$ NMR $(500$ $\left.\mathrm{MHz}, \mathrm{CD}_{3} \mathrm{OD}\right): \delta 8.07(\mathrm{~d}, J=8.5 \mathrm{~Hz}, 2 \mathrm{H}), 7.37(\mathrm{~d}, J=8.3 \mathrm{~Hz}, 2 \mathrm{H})$, 4.36 (q, $J=7.1 \mathrm{~Hz}, 2 \mathrm{H}), 3.33(\mathrm{t}, J=7.2 \mathrm{~Hz}, 2 \mathrm{H}), 1.70-1.60(\mathrm{~m}, 2 \mathrm{H})$, $1.51-1.41(\mathrm{~m}, 2 \mathrm{H}), 1.39(\mathrm{t}, J=7.1 \mathrm{~Hz}, 3 \mathrm{H}), 0.99(\mathrm{t}, J=7.4 \mathrm{~Hz}, 3 \mathrm{H})$. ${ }^{13} \mathrm{C}$ NMR (125 MHz, $\left.\mathrm{CD}_{3} \mathrm{OD}\right): \delta 167.2,156.3,141.7,132.2,129.2$, $124.3,105.6,62.3,42.9,31.8,20.9,14.6,14.0 . \mathrm{MS}(\mathrm{ESI})^{+}$calculated for $\mathrm{C}_{14} \mathrm{H}_{21} \mathrm{~N}_{3} \mathrm{O}_{2}[\mathrm{M}+\mathrm{H}]^{+}: \mathrm{m} / \mathrm{z} 264.2$, found 264.1.

1-Butyl-2-(4-vinylphenyl)guanidine hydrochloride (20)<smiles>C=Cc1ccc([NH2+]C(N)NCCCC)cc1</smiles>

20

Guanidine 20 was afforded ( $73 \mathrm{mg}, 57 \%$ ) following General Procedure $A$ as a white solid after purification by flash column chromatography $\left(\mathrm{SiO}_{2}, \mathrm{DCM} \rightarrow \mathrm{DCM} / \mathrm{MeOH}\right.$ 9:1). ${ }^{1} \mathrm{H} \mathrm{NMR}\left(500 \mathrm{MHz}, \mathrm{CD}_{3} \mathrm{OD}\right): \delta 7.53(\mathrm{~d}$, $J=7.5 \mathrm{~Hz}, 2 \mathrm{H}), 7.24(\mathrm{~d}, J=7.6 \mathrm{~Hz}, 2 \mathrm{H}), 6.76(\mathrm{dd}, J=17.5,11.0 \mathrm{~Hz}$, $1 \mathrm{H}), 5.82(\mathrm{~d}, J=17.6 \mathrm{~Hz}, 1 \mathrm{H}), 5.29(\mathrm{~d}, J=10.8 \mathrm{~Hz}, 1 \mathrm{H}), 3.30(\mathrm{t}, J=6.7$ $\mathrm{Hz}, 2 \mathrm{H}), 1.68-1.58(\mathrm{~m}, 2 \mathrm{H}), 1.49-1.38(\mathrm{~m}, 2 \mathrm{H}), 0.99(\mathrm{t}, J=7.2 \mathrm{~Hz}, 3 \mathrm{H})$. ${ }^{13} \mathrm{C}$ NMR $\left(125 \mathrm{MHz}, \mathrm{CD}_{3} \mathrm{OD}\right): \delta 156.8,138.1,137.0,135.8,128.7,126.3$, 115.1, 42.7, 31.9, 20.9, 14.0. MS (ESI)+ calculated for $\mathrm{C}_{13} \mathrm{H}_{19} \mathrm{~N}_{3}[\mathrm{M}+\mathrm{H}]^{+}$:

$\mathrm{m} / \mathrm{z} 218.2$, found 264.1

1-Butyl-2-(4-hydroxyphenyl)guanidine hydrochloride (2p)<smiles>CCCCNC(N)=[NH+]c1ccc(O)cc1</smiles>

$2 p$

Guanidine $2 p$ was afforded (118 mg, 96\%) following General Procedure $B$ as a dark brown gel after purification by flash column chromatography $\left(\mathrm{SiO}_{2}, \mathrm{DCM} \rightarrow \mathrm{DCM} / \mathrm{MeOH} 9: 1\right) .{ }^{1} \mathrm{H}$ NMR $\left(500 \mathrm{MHz}, \mathrm{CD}_{3} \mathrm{OD}\right): \delta 7.10(\mathrm{~d}$, $J=8.7 \mathrm{~Hz}, 2 \mathrm{H}), 6.88(\mathrm{~d}, J=8.7 \mathrm{~Hz}, 2 \mathrm{H}), 3.27$ (t, $J=7.0 \mathrm{~Hz}, 2 \mathrm{H}), 1.67-$ $1.56(\mathrm{~m}, 2 \mathrm{H}), 1.48-1.37(\mathrm{~m}, 2 \mathrm{H}), 0.98(\mathrm{t}, J=7.4 \mathrm{~Hz}, 3 \mathrm{H}) .{ }^{13} \mathrm{C}$ NMR $(125$ $\left.\mathrm{MHz}, \mathrm{CD}_{3} \mathrm{OD}\right): \delta 158.6,157.3,129.1,127.0,117.5,42.5,32.0,20.8,14.0$. MS $(E S I)^{+}$calculated for $\mathrm{C}_{11} \mathrm{H}_{17} \mathrm{~N}_{3} \mathrm{O}[\mathrm{M}+\mathrm{H}]^{+}: \mathrm{m} / \mathrm{z} 208.1$, found 208.1 .

2-(Benzo[d][1,3]dioxol-5-yl)-1-butylguanidine hydrochloride (2q)<smiles>CCCCNC(N)=[NH+]c1ccc2c(c1)OCO2</smiles>

$2 q$
Guanidine 2q was afforded (108 mg, 80\%) following General Procedure $A$ as a brown gel after purification by flash column chromatography $\left(\mathrm{SiO}_{2}\right.$, $\mathrm{DCM} \rightarrow \mathrm{DCM} / \mathrm{MeOH}$ 9:1). ${ }^{1} \mathrm{H}$ NMR $\left(500 \mathrm{MHz}, \mathrm{CD}_{3} \mathrm{OD}\right): \delta 6.88(\mathrm{~d}, J=$ $8.1 \mathrm{~Hz}, 1 \mathrm{H}), 6.83-6.73(\mathrm{~m}, 2 \mathrm{H}), 6.03(\mathrm{~s}, 2 \mathrm{H}), 3.28(\mathrm{t}, J=7.1 \mathrm{~Hz}, 2 \mathrm{H})$, $1.68-1.57(\mathrm{~m}, 2 \mathrm{H}), 1.49-1.37(\mathrm{~m}, 2 \mathrm{H}), 0.98(\mathrm{t}, J=7.4 \mathrm{~Hz}, 3 \mathrm{H}) .{ }^{13} \mathrm{C} N M R$ (125 MHz, $\left.\mathrm{CD}_{3} \mathrm{OD}\right): \delta 157.3,150.1,148.8,129.5,121.2,109.7,108.5$, 103.3, 42.6, 31.9, 20.9, 14.0. MS (ESI) calculated for $\mathrm{C}_{12} \mathrm{H}_{17} \mathrm{~N}_{3} \mathrm{O}_{2}$ $[\mathrm{M}+\mathrm{H}]^{+}: \mathrm{m} / \mathrm{z}$ 236.1, found 236.1 . 
1-Butyl-2-(naphthalen-1-yl)guanidine hydrochloride (2r)<smiles>CCCCNC(N)=[NH+]c1cccc2ccccc12</smiles>

$2 r$

Guanidine $2 \mathrm{r}$ was afforded (57 $\mathrm{mg}, 41 \%$ ) following General Procedure A as a pale brown gel after purification by flash column chromatography $\left(\mathrm{SiO}_{2}\right.$, $\mathrm{DCM} \rightarrow \mathrm{DCM} / \mathrm{MeOH}$ 9:1). ${ }^{1} \mathrm{H} \mathrm{NMR}\left(500 \mathrm{MHz}, \mathrm{CD}_{3} \mathrm{OD}\right): \delta$ 8.05-7.92 (m, $3 \mathrm{H}$ ), 7.68-7.54 (m, 3H), 7.50 (d, $J=7.2 \mathrm{~Hz}, 1 \mathrm{H}), 3.33$ (app s, 2H), 1.71$1.54(\mathrm{~m}, 2 \mathrm{H}), 1.49-1.35(\mathrm{~m}, 2 \mathrm{H}), 0.98(\mathrm{t}, J=7.3 \mathrm{~Hz}, 3 \mathrm{H}) .{ }^{13} \mathrm{C}$ NMR $(125$ $\left.\mathrm{MHz}, \mathrm{CD}_{3} \mathrm{OD}\right): \delta 157.7,136.2,131.6,130.3,129.7,128.4,127.9,127.0$, 127.0, 122.9, 42.7, 32.0, 20.9, 14.0. MS (ESI)+ calculated for $\mathrm{C}_{15} \mathrm{H}_{19} \mathrm{~N}_{3}$ $[\mathrm{M}+\mathrm{H}]^{+}: \mathrm{m} / \mathrm{z} 242.2$, found 242.2 .

1-Benzyl-2-phenylguanidine hydrochloride (2s)<smiles></smiles>

2s

Guanidine 2s was afforded (128 mg, 98\%) following General Procedure A as a pale yellow solid after purification by flash column chromatography $\left(\mathrm{SiO}_{2}, \mathrm{DCM} \rightarrow \mathrm{DCM} / \mathrm{MeOH}\right.$ 9:1). ${ }^{1} \mathrm{H}$ NMR (500 MHz, CD $\left.{ }_{3} \mathrm{OD}\right): \delta 7.50-7.44$ (m, 2H), 7.43-7.38 (m, 4H), 7.37-7.32 (m, 2H), 7.31-7.27 (m, 2H), 4.56 (s, $2 \mathrm{H}) .{ }^{13} \mathrm{C}$ NMR (125 MHz, CD $\left.{ }_{3} \mathrm{OD}\right): \delta 156.9,137.5,136.2,131.0,129.9$, 129.0, 128.6, 128.4, 126.5. MS (ESI) ${ }^{+}$calculated for $\mathrm{C}_{14} \mathrm{H}_{15} \mathrm{~N}_{3}[\mathrm{M}+\mathrm{H}]^{+}: \mathrm{m} / \mathrm{z}$ 226.1 , found 226.1 .

1-Cyclohexyl-2-phenylguanidine hydrochloride (2t)<smiles>NC(NC1CCCCC1)=[NH+]c1ccccc1</smiles>

$2 t$

Guanidine 2t was afforded (51 mg, 40\%) following General Procedure $A$ as a white solid after purification by flash column chromatography $\left(\mathrm{SiO}_{2}, \mathrm{DCM} \rightarrow\right.$ DCM/MeOH 9:1). ${ }^{1} \mathrm{H}$ NMR (500 MHz, CD $\left.{ }_{3} \mathrm{OD}\right): \delta$ 7.57-7.40 (m, 2H), 7.40$7.31(\mathrm{~m}, 1 \mathrm{H}), 7.28(\mathrm{~d}, J=7.1 \mathrm{~Hz}, 2 \mathrm{H}), 3.65-3.49(\mathrm{~m}, 1 \mathrm{H}), 2.08-1.92(\mathrm{~m}, 2 \mathrm{H})$, $1.87-1.73(\mathrm{~m}, 2 \mathrm{H}), 1.71-1.60(\mathrm{~m}, 1 \mathrm{H}), 1.51-1.32(\mathrm{~m}, 4 \mathrm{H}), 1.32-1.18(\mathrm{~m}, 1 \mathrm{H})$. ${ }^{13} \mathrm{C}$ NMR (125 MHz, CD $\left.{ }_{3} \mathrm{OD}\right): \delta 155.8,136.5,131.0,128.4,126.3,52.3,33.6$, 26.2, 25.7. MS (ESI) calculated for $\mathrm{C}_{13} \mathrm{H}_{19} \mathrm{~N}_{3}[\mathrm{M}+\mathrm{H}]^{+}: \mathrm{m} / \mathrm{z}$ 218.2, found 218.2.

1,2-Diphenylguanidine hydrochloride $(\mathbf{2 u})$<smiles>NC(Nc1ccccc1)=[NH+]c1ccccc1</smiles>

$2 u$
Guanidine $\mathbf{2 u}$ was afforded (108 $\mathrm{mg}, 87 \%$ ) following General Procedure $A$ as a pale yellow solid after purification by flash column chromatography $\left(\mathrm{SiO}_{2}\right.$, $\mathrm{DCM} \rightarrow \mathrm{DCM} / \mathrm{MeOH}$ 9:1). ${ }^{1} \mathrm{H}$ NMR $\left(500 \mathrm{MHz}, \mathrm{CD}_{3} \mathrm{OD}\right): 87.53-7.45(\mathrm{~m}, 4 \mathrm{H})$, 7.42-7.33 (m, 6H). ${ }^{13} \mathrm{C}$ NMR (125 MHz, $\left.\mathrm{CD}_{3} \mathrm{OD}\right): \delta 156.2,136.5,131.0,128.4$, 126.1. MS (ESI)+ calculated for $\mathrm{C}_{13} \mathrm{H}_{13} \mathrm{~N}_{3}[\mathrm{M}+\mathrm{H}]^{+}: \mathrm{m} / \mathrm{z} 212.1$, found 212.1. 
1-(4-Methoxyphenyl)-2-phenylguanidine hydrochloride (2v)<smiles>COc1ccc(NC(N)=[NH+]c2ccccc2)cc1</smiles>

2v

Guanidine $2 v$ was afforded (78 $\mathrm{mg}, 56 \%$ ) following General Procedure $A$ as a pale yellow gel after purification by flash column chromatography $\left(\mathrm{SiO}_{2}, \mathrm{DCM} \rightarrow \mathrm{DCM} / \mathrm{MeOH}\right.$ 9:1). ${ }^{1} \mathrm{H} \mathrm{NMR}(500 \mathrm{MHz}$, $\left.\mathrm{CD}_{3} \mathrm{OD}\right): \delta$ 7.55-7.44 (m, 2H), 7.42-7.33 (m, 3H), $7.30(\mathrm{~d}, J=8.8 \mathrm{~Hz}$, $2 \mathrm{H}), 7.04(\mathrm{~d}, J=8.8 \mathrm{~Hz}, 2 \mathrm{H}), 3.83(\mathrm{~s}, 3 \mathrm{H}) .{ }^{13} \mathrm{C}$ NMR $\left(125 \mathrm{MHz}, \mathrm{CD}_{3} \mathrm{OD}\right)$ : $\delta 160.7,156.8,136.3,131.0,128.6,128.3,126.4,116.2,56.1 . \mathrm{MS}$ $(\mathrm{ESI})^{+}$calculated for $\mathrm{C}_{14} \mathrm{H}_{15} \mathrm{~N}_{3} \mathrm{O}[\mathrm{M}+\mathrm{H}]^{+}: \mathrm{m} / \mathrm{z} 242.1$, found 242.1 .

2-Phenyl-1-(p-tolyl)guanidine hydrochloride (2w)<smiles>Cc1ccc(NC(N)=[NH+]c2ccccc2)cc1</smiles>

2w

Guanidine 2w was afforded (103 mg, 79\%) following General Procedure $A$ as a pale yellow gel after purification by flash column chromatography $\left(\mathrm{SiO}_{2}, \mathrm{DCM} \rightarrow \mathrm{DCM} / \mathrm{MeOH}\right.$ 9:1). ${ }^{1} \mathrm{H}$ NMR $\left(500 \mathrm{MHz}, \mathrm{CD}_{3} \mathrm{OD}\right): \delta 7.53-$ $7.45(\mathrm{~m}, 2 \mathrm{H}), 7.40-7.32(\mathrm{~m}, 3 \mathrm{H}), 7.30(\mathrm{~d}, J=7.9 \mathrm{~Hz}, 2 \mathrm{H}), 7.26(\mathrm{~d}, J=$ $8.0 \mathrm{~Hz}, 2 \mathrm{H}), 2.38(\mathrm{~s}, 3 \mathrm{H}) .{ }^{13} \mathrm{C}$ NMR $\left(125 \mathrm{MHz}, \mathrm{CD}_{3} \mathrm{OD}\right): \delta 156.4,138.8$, $136.4,133.5,131.5,131.0,128.5,126.4,126.2,21.1 . \mathrm{MS}(\mathrm{ESI})^{+}$ calculated for $\mathrm{C}_{14} \mathrm{H}_{15} \mathrm{~N}_{3}[\mathrm{M}+\mathrm{H}]^{+}: \mathrm{m} / \mathrm{z} 226.1$, found 226.1 .

1-(4-Chlorophenyl)-2-phenylguanidine hydrochloride (2x)<smiles>NC(Nc1ccc(Cl)cc1)=[NH+]c1ccccc1</smiles>

$2 x$
Guanidine 2x was afforded (125 mg, 89\%) following General Procedure $A$ as a pale yellow gel after purification by flash column chromatography $\left(\mathrm{SiO}_{2}, \mathrm{DCM} \rightarrow \mathrm{DCM} / \mathrm{MeOH} 9: 1\right)$. ${ }^{1} \mathrm{H} \mathrm{NMR}\left(500 \mathrm{MHz}, \mathrm{CD}_{3} \mathrm{OD}\right): \delta 7.52-$ $7.44(\mathrm{~m}, 4 \mathrm{H}), 7.41-7.31(\mathrm{~m}, 5 \mathrm{H}) .{ }^{13} \mathrm{C}$ NMR (125 MHz, CD $\left.{ }_{3} \mathrm{OD}\right): \delta 213.3$, $156.0,136.7,135.8,133.5,130.9,128.3,127.6,126.0 . \mathrm{MS}(\mathrm{ESI})^{+}$ calculated for $\mathrm{C}_{13} \mathrm{H}_{12} \mathrm{CIN} 3[\mathrm{M}+\mathrm{H}]^{+}: \mathrm{m} / \mathrm{z} 246.1$, found 246.1 .

Guanidine 2y<smiles>CCCCNC(=O)[C@H](CCCNC(N)=[NH+]c1ccccc1)NCCCC</smiles>

Following General Procedure A, GCDI $1 \mathrm{~h}$ (121 mg, $0.277 \mathrm{mmol})$, aniline (0.0775 $\mathrm{ml}, 0.832 \mathrm{mmol}), \operatorname{TFA}(0.0215 \mathrm{ml}, 0.277 \mathrm{mmol}), \mathrm{MeCN}(1.11 \mathrm{ml}), 0.5 \mathrm{M}$ aqueous $\mathrm{NaOH}$ solution $(5.54 \mathrm{ml})$ and $0.5 \mathrm{M} \mathrm{HCl}$ solution in $\mathrm{MeOH}(10.0 \mathrm{ml})$ yielded guanidine $2 \mathbf{y}(43.8 \mathrm{mg}, 41 \%)$ as a colorless gel after purification by flash column chromatography $\left(\mathrm{SiO}_{2}, \mathrm{DCM} \rightarrow \mathrm{DCM} / \mathrm{MeOH} 8: 2\right) .{ }^{1} \mathrm{H} \mathrm{NMR}\left(500 \mathrm{MHz}, \mathrm{CD}_{3} \mathrm{OD}\right): \delta$ 7.51-7.44 (m, 2H), 7.38-7.32 (m, 1H), $7.29(\mathrm{~d}, J=7.6 \mathrm{~Hz}, 2 \mathrm{H}), 4.35-4.29(\mathrm{~m}$, $1 \mathrm{H}), 3.33(\mathrm{t}, J=6.8 \mathrm{~Hz}, 2 \mathrm{H}), 3.19(\mathrm{t}, J=7.1 \mathrm{~Hz}, 2 \mathrm{H}), 2.02(\mathrm{~s}, 3 \mathrm{H}), 1.92-1.82(\mathrm{~m}$, $1 \mathrm{H}), 1.78-1.59(\mathrm{~m}, 3 \mathrm{H}), 1.54-1.45(\mathrm{~m}, 2 \mathrm{H}), 1.40-1.30(\mathrm{~m}, 2 \mathrm{H}), 0.93(\mathrm{t}, J=7.4 \mathrm{~Hz}$, $3 \mathrm{H}) .{ }^{13} \mathrm{C}$ NMR $\left(125 \mathrm{MHz}, \mathrm{CD}_{3} \mathrm{OD}\right): \delta$ 173.9, 173.4, 156.8, 136.3, 131.1, 128.5, $126.5,64.2,54.6,42.4,40.1,32.5,30.3,26.4,22.6,21.0,14.1 . \mathrm{MS}(\mathrm{ESI})^{+}$ calculated for $\mathrm{C}_{18} \mathrm{H}_{29} \mathrm{~N}_{5} \mathrm{O}_{2}[\mathrm{M}+\mathrm{H}]^{+}: \mathrm{m} / \mathrm{z} 348.2$, found 348.2. 


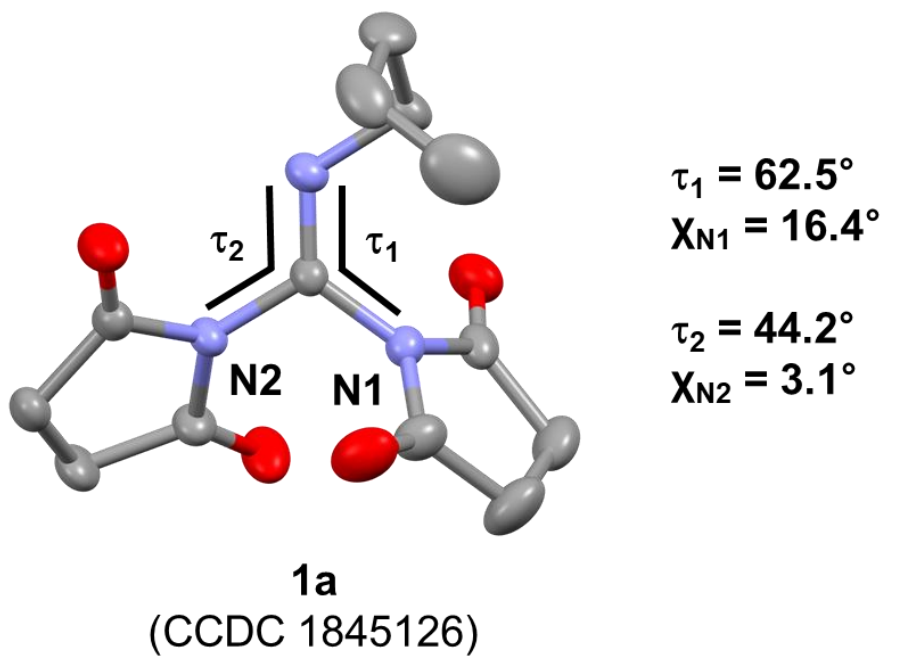

Figure S1. The Winkler-Dunitz distortion parameters from the X-ray crystal structure of GCDI 1a (CCDC $1845126)^{1}$. Thermal ellipsoids represent $50 \%$ probability. 


\section{F. ${ }^{15} \mathrm{~N}$ Incorporation Studies}<smiles>CCCCN=C(N1C(=O)CCC1=O)N1C(=O)CCC1=O</smiles>

$1 \mathrm{a}$<smiles>[SnH3]c1ccccc1</smiles>

3.0 eq.

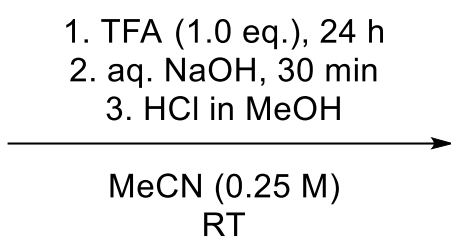<smiles></smiles>

$3,92 \%$

Following General Procedure A, ${ }^{15} \mathrm{~N}$-incorporated guanidine 3 was synthesized as follows:

To a stirred solution of GCDI 1a (140 mg, $0.500 \mathrm{mmol}, 1.00$ eq.) and TFA ( $0.0392 \mathrm{ml}, 0.500 \mathrm{mmol}, 1.00$ eq.) in $\mathrm{MeCN}(2.00 \mathrm{ml}),{ }^{15} \mathrm{~N}$-aniline $(0.139 \mathrm{ml}, 1.50 \mathrm{mmol}, 3.00$ eq.) was added, then the reaction mixture was stirred at ambient temperature. After $24 \mathrm{~h}$ reaction, the mixture was quenched by the addition of $0.5 \mathrm{M}$ aqueous $\mathrm{NaOH}$ solution $(10.0 \mathrm{ml})$ and further stirred for $30 \mathrm{~min}$. The reaction mixture was concentrated under reduced pressure, diluted with ethyl acetate, then filtered. Subsequently, $0.5 \mathrm{M} \mathrm{HCl}$ solution in $\mathrm{MeOH}(15.0 \mathrm{ml})$ was added to the filtrate and the mixture was concentrated under reduced pressure. The residue was dissolved in DCM, then filtered. ${ }^{15} \mathrm{~N}$-incorporated guanidine was afforded $(105.7 \mathrm{mg}, 92 \%)$ as a yellow gel after purification by flash column chromatography $\left(\mathrm{SiO}_{2}, \mathrm{DCM} \rightarrow\right.$ $\mathrm{DCM} / \mathrm{MeOH}$ 9:1). Incorporation of ${ }^{15} \mathrm{~N}$ isotope was confirmed by ${ }^{1} \mathrm{H}-{ }^{15} \mathrm{~N} \mathrm{HMBC} \mathrm{NMR}$ and HRMS. ${ }^{1} \mathrm{H}-$ ${ }^{15} \mathrm{~N}$ HMBC NMR analysis was performed at National Center for Inter-University Research Facilities at Seoul National University. ${ }^{1} \mathrm{H}$ NMR $\left(500 \mathrm{MHz}, \mathrm{CD}_{3} \mathrm{OD}\right): \delta$ 7.50-7.42 $(\mathrm{m}, 2 \mathrm{H}), 7.37-7.30(\mathrm{~m}, 1 \mathrm{H}), 7.27$ (d, $J=7.6 \mathrm{~Hz}, 2 \mathrm{H}), 3.30(\mathrm{t}, J=6.9 \mathrm{~Hz}, 2 \mathrm{H}), 1.67-1.57(\mathrm{~m}, 2 \mathrm{H}), 1.49-1.38(\mathrm{~m}, 2 \mathrm{H}), 0.97(\mathrm{t}, J=7.4 \mathrm{~Hz}$, 3H). ${ }^{13} \mathrm{C}$ NMR (125 MHz, CD $\left.{ }_{3} \mathrm{OD}\right): \delta$ 156.9, 156.7, 136.4, 136.3, 131.0, 131.0, 128.4, 126.4, 42.7, 31.9, 20.9, 14.0. HRMS $(E S I)+$ calculated for $\mathrm{C}_{11} \mathrm{H}_{18} \mathrm{~N}_{2}{ }^{15} \mathrm{~N}^{+}[\mathrm{M}+\mathrm{H}]^{+}$: $\mathrm{m} / \mathrm{z}$ 193.1471, found 193.1460 . 

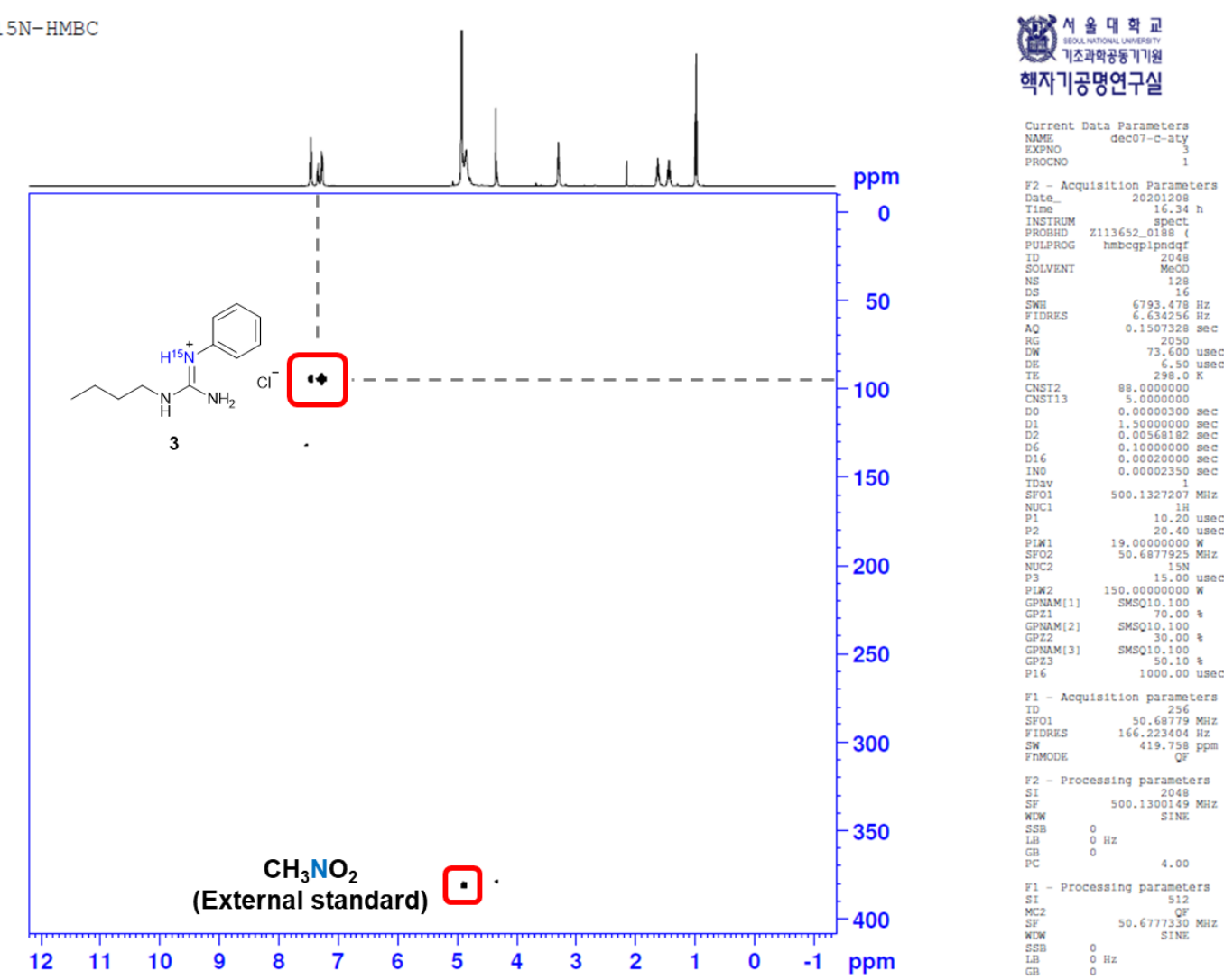

Figure S2. ${ }^{1} \mathrm{H}-{ }^{15} \mathrm{~N}$ HMBC NMR spectrum of ${ }^{15} \mathrm{~N}$-incorporated guanidine 3 with $\mathrm{CH}_{3} \mathrm{NO}_{2}$ external standard. 


\section{G. Hammett Plot Studies}<smiles></smiles><smiles>[R]c1ccc(N)cc1</smiles>

1. TFA (1.0 eq.), $24 \mathrm{~h}$

2. aq. $\mathrm{NaOH}, 30 \mathrm{~min}$<smiles>[R]c1ccc(/N=C(\N)NCCCC)cc1</smiles>

$\mathrm{MeCN}(0.25 \mathrm{M})$<smiles>CCCCN/C(N)=N/c1ccccc1</smiles>

1a

To a stirred solution of GCDI 1a (140 mg, $0.500 \mathrm{mmol}, 1.00$ eq.) and TFA ( $0.0392 \mathrm{ml}, 0.500 \mathrm{mmol}, 1.00$ eq.) in $\mathrm{MeCN}(2.00 \mathrm{ml}$ ), two amine substrates (each $0.750 \mathrm{mmol}, 1.50$ eq.) were added, then the reaction mixture was stirred at ambient temperature. After $24 \mathrm{~h}$ reaction, the mixture was quenched by the addition of a $0.5 \mathrm{M}$ aqueous $\mathrm{NaOH}$ solution $(10.0 \mathrm{ml})$ and further stirred for $30 \mathrm{~min}$. The reaction mixture was concentrated under reduced pressure, diluted with ethyl acetate, then filtered. The molar ratio of two guanidine products was determined by ${ }^{1} \mathrm{H}$ NMR.

Table S3. Competition Experiments with Two Different Amine Nucleophiles

\begin{tabular}{ccccc}
\hline Entry & $\mathbf{R}$ & $\boldsymbol{\sigma}$ & $\sigma^{+}$ & $\begin{array}{c}\text { Product ratio } \\
(\mathrm{R}: \mathrm{H})^{\mathbf{a}}\end{array}$ \\
\hline $\mathbf{1}$ & $\mathrm{OMe}$ & -0.268 & -0.78 & 2.35 \\
\hline $\mathbf{2}$ & $\mathrm{Me}$ & -0.17 & -0.31 & 1.68 \\
\hline $\mathbf{3}$ & $\mathrm{Cl}$ & 0.227 & 0.11 & 0.548 \\
\hline $\mathbf{4}$ & $\mathrm{CF}_{3}$ & 0.54 & 0.61 & 0.0818 \\
\hline
\end{tabular}

a Determined by ${ }^{1} \mathrm{H}$ NMR. 


\section{H. Natural Bond Orbital (NBO) Analysis}

All of the calculations were performed using the Gaussian 09W package ${ }^{4}$. Geometry optimizations were performed at the B3LYP/6-311+G(d,p) level of theory in the gas phase. Vibrational analysis was performed at each stationary point found, and the absence of imaginary frequencies was used to characterize the structures as minima on the potential energy surface. All of the optimized geometries were verified as minima (no imaginary frequencies). Electronic and thermal energies were calculated for all structures. Energetic parameters were calculated under standard conditions $(298.15 \mathrm{~K}$ and 1 atm). The population analysis has also been performed by the natural bond orbital method at B3LYP/6$311+G(d, p)$ level of theory using natural bond orbital (NBO) program under the Gaussian 09W program package. Natural bond orbital (NBO) calculation of the protonated and neutral form of the model GCDI $1 \mathrm{~m}$ and the intermediate $\mathbf{1 7}$ was performed at the level of B3LYP/6-311+G(d,p). Graphical structures were visualized with Mercury.<smiles>CN=C(N1C(=O)CCC1=O)N1C(=O)CCC1=O</smiles>

$1 \mathrm{~m}$<smiles></smiles>

$\left[1 \mathrm{~m}+\mathrm{H}^{+}\right]\left[\mathrm{CF}_{3} \mathrm{COO}^{-}\right]$<smiles>CN/C(=N\c1ccccc1)N1C(=O)CCC1=O</smiles>

17<smiles>CNC(=N[IH+])N1C(=O)CCC1=O</smiles>

$\mathrm{CF}_{3} \mathrm{COO}$

$\left[17+\mathrm{H}^{+}\right]\left[\mathrm{CF}_{3} \mathrm{COO}^{-}\right]$

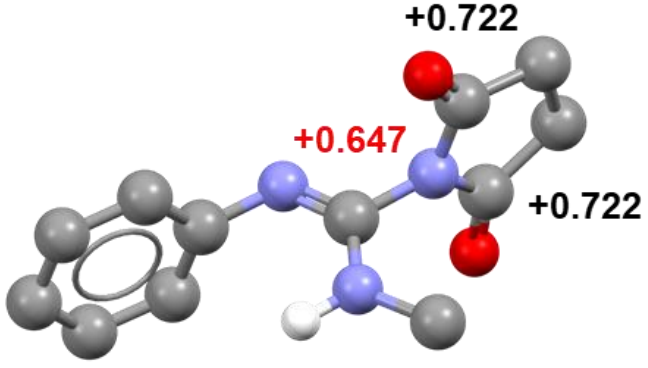

17

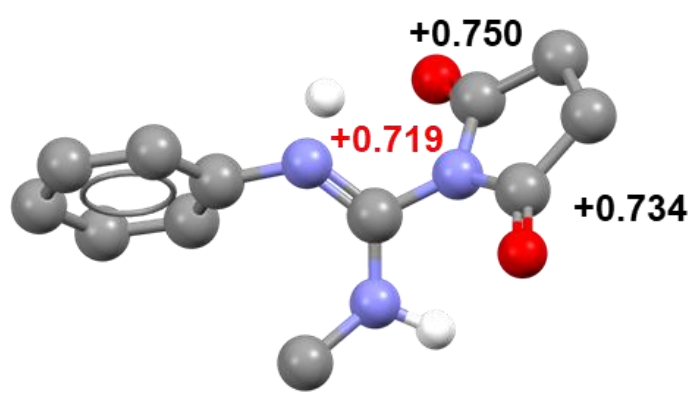

$\left[17+\mathrm{H}^{+}\right]\left[\mathrm{CF}_{3} \mathrm{COO}^{-}\right]$

Figure S3. The optimized structures with the NBO charges of the neutral and protonated form of $\mathbf{I 7 .}$ The NBO charges of the guanidine center (red number) and the imide carbonyl carbons (black number) are indicated in both structures. The hydrogen atoms bonded to carbon and $\mathrm{CF}_{3} \mathrm{COO}^{-}$were omitted for clarity. 
Table S4. The optimized atomic coordinates of methyl GCDI (1m)

\begin{tabular}{|l|r|r|r|}
\hline Atom & $\mathbf{X}$ & $\mathbf{Y}$ & $\mathbf{Z}$ \\
\hline $\mathbf{N}$ & 0.004776 & 2.172099 & -0.23383 \\
\hline $\mathbf{C}$ & 0.003551 & 0.927184 & -0.09475 \\
\hline $\mathbf{C}$ & -1.15495 & 2.989675 & 0.047858 \\
\hline $\mathbf{N}$ & -1.12092 & 0.096163 & 0.217764 \\
\hline $\mathbf{N}$ & 1.219459 & 0.202719 & -0.24937 \\
\hline $\mathbf{C}$ & 1.334054 & -0.99022 & -0.9785 \\
\hline $\mathbf{C}$ & 2.769825 & -1.4736 & -0.86678 \\
\hline $\mathbf{C}$ & 3.484014 & -0.44687 & 0.017002 \\
\hline $\mathbf{C}$ & 2.422473 & 0.566676 & 0.419506 \\
\hline $\mathbf{C}$ & -1.09953 & -0.9136 & 1.201534 \\
\hline $\mathbf{C}$ & -2.40732 & -1.68206 & 1.109494 \\
\hline $\mathbf{C}$ & -3.15685 & -1.08057 & -0.08373 \\
\hline $\mathbf{C}$ & -2.27224 & 0.039952 & -0.60524 \\
\hline $\mathbf{O}$ & 2.549078 & 1.492108 & 1.168275 \\
\hline $\mathbf{O}$ & 0.432411 & -1.5234 & -1.57957 \\
\hline $\mathbf{O}$ & -2.47927 & 0.761122 & -1.54293 \\
\hline $\mathbf{O}$ & -0.19024 & -1.10483 & 1.967896 \\
\hline $\mathbf{H}$ & -0.81014 & 3.904184 & 0.53425 \\
\hline $\mathbf{H}$ & -1.62684 & 3.265957 & -0.89817 \\
\hline $\mathbf{H}$ & -1.90551 & 2.50323 & 0.680227 \\
\hline $\mathbf{H}$ & 2.757212 & -2.47839 & -0.43968 \\
\hline $\mathbf{H}$ & 3.185242 & -1.55525 & -1.87303 \\
\hline $\mathbf{H}$ & 4.284951 & 0.087088 & -0.49895 \\
\hline $\mathbf{H}$ & 3.910769 & -0.87245 & 0.926417 \\
\hline $\mathbf{H}$ & -2.17215 & -2.74086 & 0.988526 \\
\hline $\mathbf{H}$ & -2.93777 & -1.57464 & 2.058061 \\
\hline $\mathbf{H}$ & -4.13369 & -0.66587 & 0.172456 \\
\hline $\mathbf{H}$ & -3.30922 & -1.78935 & -0.89977 \\
\hline & & & \\
\hline
\end{tabular}

Table S5. The optimized atomic coordinates of $\left[1 \mathrm{~m}+\mathrm{H}^{+}\right]\left[\mathrm{CF}_{3} \mathrm{COO}^{-}\right]$

\begin{tabular}{|l|r|r|r|}
\hline Atom & $\mathbf{X}$ & $\mathbf{Y}$ & $\mathbf{Z}$ \\
\hline $\mathbf{N}$ & -0.96943 & -0.22877 & 2.086321 \\
\hline $\mathbf{C}$ & -1.24784 & -0.22466 & 0.810039 \\
\hline $\mathbf{C}$ & 0.025149 & -1.07422 & 2.758618 \\
\hline $\mathbf{N}$ & -0.97347 & -1.28543 & -0.01655 \\
\hline
\end{tabular}




\begin{tabular}{|l|r|r|r|}
\hline $\mathbf{N}$ & -1.86135 & 0.885174 & 0.268621 \\
\hline $\mathbf{C}$ & -2.68121 & 0.901369 & -0.9159 \\
\hline $\mathbf{C}$ & -2.71992 & 2.333952 & -1.40498 \\
\hline $\mathbf{C}$ & -1.92982 & 3.160008 & -0.37914 \\
\hline $\mathbf{C}$ & -1.63444 & 2.213737 & 0.756585 \\
\hline $\mathbf{C}$ & -0.42719 & -1.14643 & -1.36008 \\
\hline $\mathbf{C}$ & -0.01747 & -2.52367 & -1.82097 \\
\hline $\mathbf{C}$ & -0.33206 & -3.47666 & -0.66716 \\
\hline $\mathbf{C}$ & -1.03825 & -2.65519 & 0.385653 \\
\hline $\mathbf{O}$ & -1.32048 & 2.465214 & 1.888825 \\
\hline $\mathbf{O}$ & -3.25964 & -0.05258 & -1.34499 \\
\hline $\mathbf{O}$ & -1.58653 & -3.03492 & 1.383753 \\
\hline $\mathbf{O}$ & -0.39716 & -0.12052 & -1.96916 \\
\hline $\mathbf{H}$ & -1.21319 & 0.645163 & 2.551727 \\
\hline $\mathbf{H}$ & 0.496417 & -0.4602 & 3.524445 \\
\hline $\mathbf{H}$ & -0.46228 & -1.93716 & 3.211302 \\
\hline $\mathbf{H}$ & 0.780122 & -1.38831 & 2.036298 \\
\hline $\mathbf{H}$ & -2.27748 & 2.350273 & -2.40253 \\
\hline $\mathbf{H}$ & -3.76276 & 2.642253 & -1.49967 \\
\hline $\mathbf{H}$ & -2.45407 & 4.037906 & -0.00251 \\
\hline $\mathbf{H}$ & -0.96052 & 3.484744 & -0.76845 \\
\hline $\mathbf{H}$ & -0.57564 & -2.75157 & -2.73218 \\
\hline $\mathbf{H}$ & 1.043509 & -2.48028 & -2.06505 \\
\hline $\mathbf{H}$ & 0.576516 & -3.86812 & -0.20274 \\
\hline $\mathbf{H}$ & -0.96176 & -4.32448 & -0.93931 \\
\hline $\mathbf{F}$ & 3.928792 & 0.215109 & -1.34802 \\
\hline $\mathbf{C}$ & 3.399739 & 0.751746 & -0.22516 \\
\hline $\mathbf{F}$ & 4.275327 & 0.506541 & 0.78372 \\
\hline $\mathbf{C}$ & 3.370717 & 2.086465 & -0.40419 \\
\hline $\mathbf{O}$ & 1.998014 & 0.139093 & 0.109151 \\
\hline & 1.94517 & -1.10777 & 0.046628 \\
\hline & & & \\
\hline
\end{tabular}

Table S6. The optimized atomic coordinates of $\mathbf{1 7}$

\begin{tabular}{|l|r|r|r|}
\hline Atom & $\mathbf{X}$ & $\mathbf{Y}$ & $\mathbf{Z}$ \\
\hline $\mathbf{N}$ & -0.0353 & 0.029335 & -1.56583 \\
\hline $\mathbf{C}$ & 0.252752 & 0.00249 & -0.2376 \\
\hline
\end{tabular}




\begin{tabular}{|l|r|r|r|}
\hline $\mathbf{C}$ & 0.933777 & 0.041978 & -2.6523 \\
\hline $\mathbf{N}$ & 1.660805 & -0.001 & 0.071243 \\
\hline $\mathbf{C}$ & 2.382795 & 1.171432 & 0.341468 \\
\hline $\mathbf{C}$ & 3.765526 & 0.755067 & 0.825888 \\
\hline $\mathbf{C}$ & 3.769296 & -0.779 & 0.791948 \\
\hline $\mathbf{O}$ & 2.389605 & -1.18037 & 0.286658 \\
\hline $\mathbf{O}$ & 1.975919 & -2.2928 & 0.083132 \\
\hline $\mathbf{N}$ & 1.962334 & 2.289627 & 0.190561 \\
\hline $\mathbf{C}$ & -0.55216 & -0.0194 & 0.747849 \\
\hline $\mathbf{C}$ & -1.94361 & -0.01214 & 0.507762 \\
\hline $\mathbf{C}$ & -2.64111 & 1.200399 & 0.408254 \\
\hline $\mathbf{C}$ & -4.02387 & 1.201454 & 0.237512 \\
\hline $\mathbf{C}$ & -4.72978 & 0.000847 & 0.166809 \\
\hline $\mathbf{C}$ & -2.03888 & -1.20611 & 0.270779 \\
\hline $\mathbf{H}$ & -1.01693 & -1.21782 & 0.442451 \\
\hline $\mathbf{H}$ & 1.573395 & 0.028345 & -1.79768 \\
\hline $\mathbf{H}$ & 1.55599 & -0.85719 & -2.64995 \\
\hline $\mathbf{H}$ & 0.382967 & 0.06801 & -3.59242 \\
\hline $\mathbf{H}$ & 4.515279 & 1.210135 & 0.175871 \\
\hline $\mathbf{H}$ & 3.913168 & 1.168101 & 1.825733 \\
\hline $\mathbf{H}$ & 3.916359 & -1.23462 & 1.773261 \\
\hline $\mathbf{H}$ & 4.522976 & -1.20165 & 0.12485 \\
\hline $\mathbf{H}$ & -2.09051 & 2.131481 & 0.480635 \\
\hline $\mathbf{H}$ & -4.55147 & 2.14645 & 0.166366 \\
\hline $\mathbf{H}$ & -5.80617 & 0.005816 & 0.039831 \\
\hline $\mathbf{H}$ & -4.57826 & -2.14601 & 0.225125 \\
\hline $\mathbf{H}$ & -2.11702 & -2.15308 & 0.539891 \\
\hline & & & \\
\hline & & & \\
\hline
\end{tabular}

Table S7. The optimized atomic coordinates of $\left[\mathrm{II}^{+} \mathrm{H}^{+}\right]\left[\mathrm{CF}_{3} \mathrm{COO}^{-}\right]$

\begin{tabular}{|l|r|r|r|}
\hline Atom & $\mathbf{X}$ & $\mathbf{Y}$ & $\mathbf{Z}$ \\
\hline $\mathbf{N}$ & -1.81033 & -2.19654 & 0.940662 \\
\hline $\mathbf{C}$ & -0.99188 & -1.4187 & 0.225287 \\
\hline $\mathbf{C}$ & -2.867 & -1.73254 & 1.84294 \\
\hline $\mathbf{N}$ & 0.238165 & -1.99387 & -0.14987 \\
\hline $\mathbf{N}$ & -1.16664 & -0.17308 & -0.12972 \\
\hline $\mathbf{C}$ & 0.747847 & -1.93456 & -1.48998 \\
\hline
\end{tabular}




\begin{tabular}{|l|r|r|r|}
\hline C & 2.22251 & -2.26708 & -1.42183 \\
\hline $\mathbf{C}$ & 2.521718 & -2.57998 & 0.050129 \\
\hline $\mathbf{C}$ & 1.20603 & -2.44045 & 0.78847 \\
\hline $\mathbf{O}$ & 0.06873 & -1.70937 & -2.449 \\
\hline $\mathbf{O}$ & 0.970951 & -2.65865 & 1.948194 \\
\hline $\mathbf{C}$ & -2.38485 & 0.564572 & -0.18485 \\
\hline $\mathbf{C}$ & -3.52551 & 0.021748 & -0.7828 \\
\hline $\mathbf{C}$ & -4.68058 & 0.79388 & -0.87936 \\
\hline $\mathbf{C}$ & -4.69425 & 2.101273 & -0.39552 \\
\hline $\mathbf{C}$ & -3.54162 & 2.641793 & 0.174425 \\
\hline $\mathbf{C}$ & -2.37963 & 1.882099 & 0.280813 \\
\hline $\mathbf{H}$ & -1.46225 & -3.13016 & 1.096692 \\
\hline $\mathbf{H}$ & -2.5823 & -0.78799 & 2.306124 \\
\hline $\mathbf{H}$ & -3.81328 & -1.59792 & 1.317356 \\
\hline $\mathbf{H}$ & -2.98753 & -2.48424 & 2.621684 \\
\hline $\mathbf{H}$ & -0.20759 & 0.35084 & -0.38271 \\
\hline $\mathbf{H}$ & 2.433223 & -3.09427 & -2.10179 \\
\hline $\mathbf{H}$ & 2.760053 & -1.38829 & -1.78239 \\
\hline $\mathbf{H}$ & 3.21877 & -1.86756 & 0.496957 \\
\hline $\mathbf{H}$ & 2.919014 & -3.58229 & 0.220699 \\
\hline $\mathbf{H}$ & -3.49626 & -0.97882 & -1.19881 \\
\hline $\mathbf{H}$ & -5.56429 & 0.37698 & -1.34873 \\
\hline $\mathbf{H}$ & -5.5939 & 2.700167 & -0.47658 \\
\hline $\mathbf{H}$ & -3.54119 & 3.663617 & 0.535711 \\
\hline $\mathbf{H}$ & -1.46759 & 2.293835 & 0.701773 \\
\hline $\mathbf{C}$ & 3.079965 & 1.932494 & 0.149615 \\
\hline $\mathbf{F}$ & 3.701339 & 0.805417 & 0.60812 \\
\hline $\mathbf{F}$ & 3.498822 & 2.948353 & 0.91204 \\
\hline $\mathbf{F}$ & 3.539915 & 2.1457 & -1.10344 \\
\hline $\mathbf{O}$ & 1.528334 & 1.742222 & 0.172124 \\
\hline & 0.858394 & 2.491318 & 0.868419 \\
\hline & 1.158106 & 0.768517 & -0.56272 \\
\hline & & & \\
\hline
\end{tabular}




\section{NMR Studies of Protonation of GCDI 1a}

$70 \mathrm{mg}$ of $1 \mathrm{a}(0.25 \mathrm{mmol})$ was dissolved in $1.0 \mathrm{ml}$ of $\mathrm{CD}_{3} \mathrm{CN}$, then indicated equivalent of TFA or acetic acid was added. ${ }^{13} \mathrm{C}$ NMR spectra were recorded on Varian NMR System $500 \mathrm{MHz}$ spectrometer.

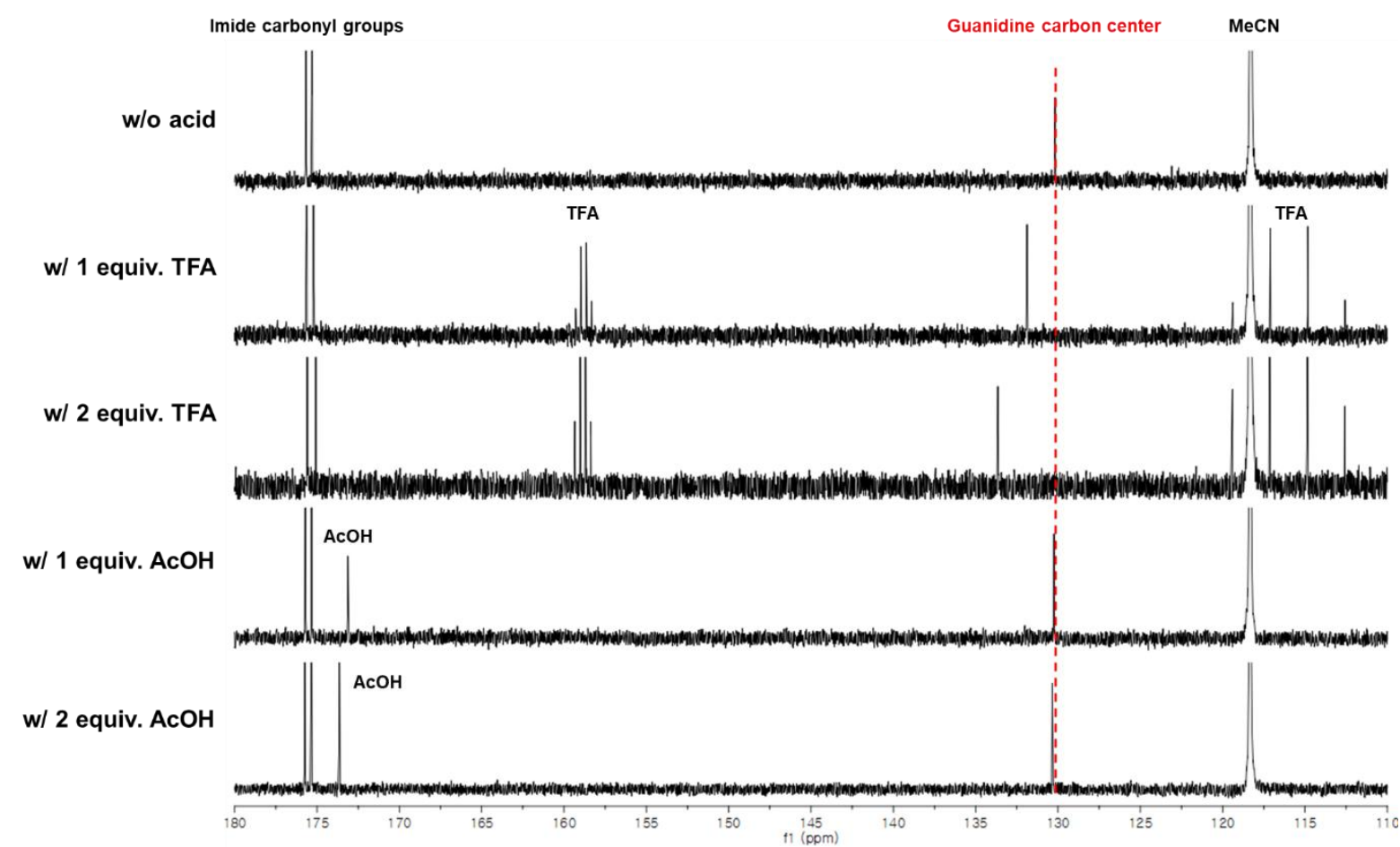

Figure S4. ${ }^{13} \mathrm{C}$ NMR spectra of GCDI 1a with and without acid additives. The guanidine carbon center peak was clearly downshifted by addition of TFA, while no shift was observed when acetic acid was added. The imide carbonyl peaks were nearly unaffected by the acid addition in both cases. 


\section{J. Investigation of Reaction Intermediates}

\section{LC-MS analysis of the reaction mixture}<smiles>CCCCN=C(N1C(=O)CCC1=O)N1C(=O)CCC1=O</smiles>

$1 \mathrm{a}$<smiles>Nc1ccccc1</smiles>

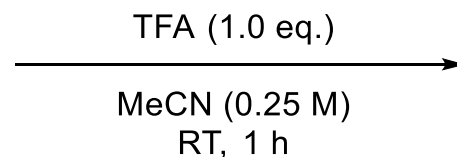

3.0 eq.

\section{LC-MS analysis}

To a stirred solution of GCDI 1a (69.8 mg, $0.250 \mathrm{mmol}, 1.00$ eq.) and TFA $(0.0196 \mathrm{ml}, 0.250 \mathrm{mmol}, 1.00$ eq.) in $\mathrm{MeCN}(1.00 \mathrm{ml})$, aniline $(0.0700 \mathrm{ml}, 0.750 \mathrm{mmol}, 3.00$ eq.) was added, then the reaction mixture was stirred at ambient temperature. After $1 \mathrm{~h}$ reaction, $10 \mu \mathrm{l}$ of the reaction mixture was diluted with 990 $\mu \mathrm{l}$ of $\mathrm{H}_{2} \mathrm{O} / \mathrm{MeCN}(\mathrm{v} / \mathrm{v}$ 1:1) solution, then analyzed by ESI/MS-(+) to detect possible reaction intermediates.

\section{Methods for LC-MS Analysis}

LC-MS chromatograms and associated mass spectra were recorded on an Agilent 6120 Quadrupole LCMS System using an ESI-TOF mass spectrometer equipped with a Shiseido UG $1205 \mu \mathrm{m}$ column. Mobile phases are: $0.1 \%$ TFA in water (solvent A) and MeCN (solvent B). Following LC-MS method was used: gradient of $0-10 \mathrm{~min}$ with $5-100 \% \mathrm{~B}$, flow rate: $1.0 \mathrm{ml} / \mathrm{min}$. 

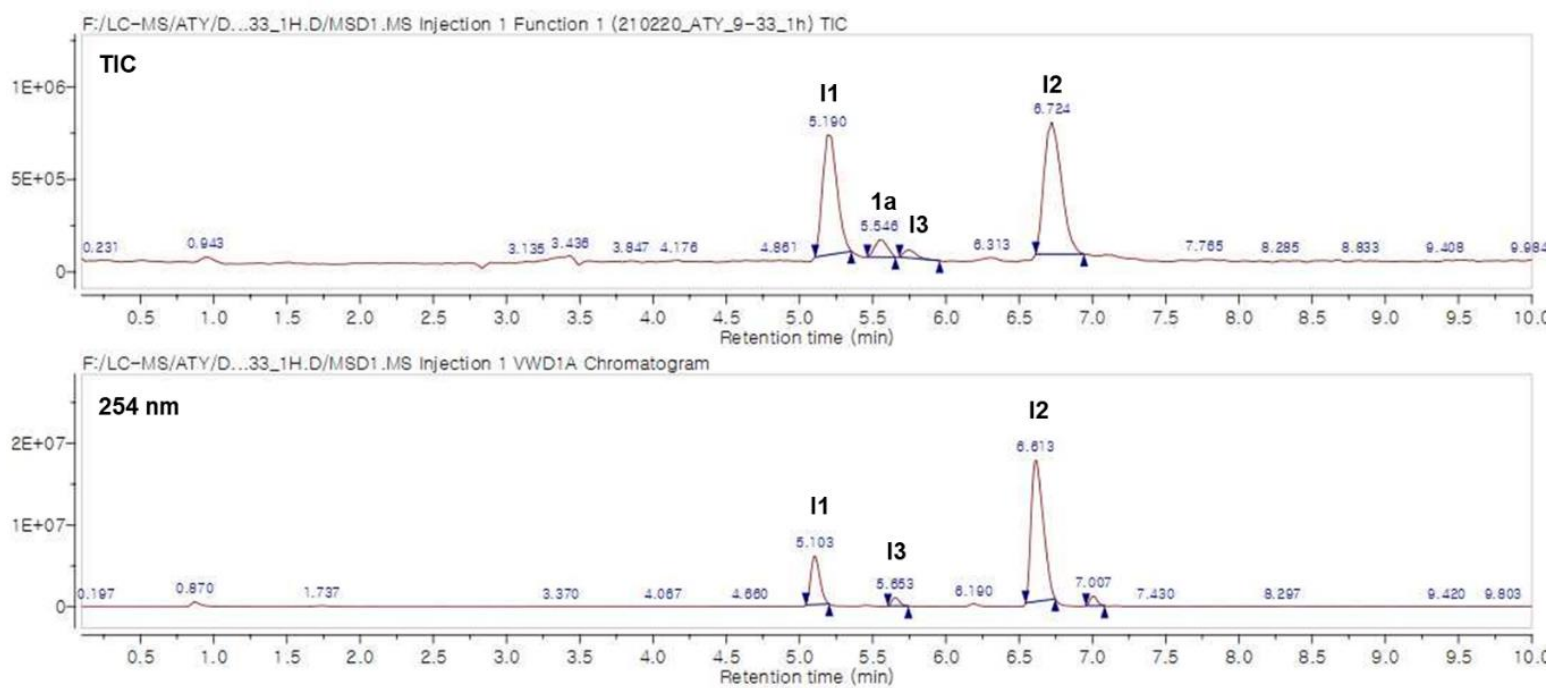

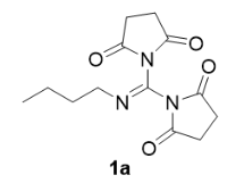

Calcd. $\mathrm{m} / \mathrm{z}=280.1[\mathrm{M}+\mathrm{H}]$ Detected $=280.1[\mathrm{M}+\mathrm{H}]^{+}$

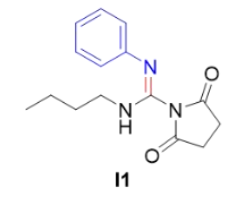

Calcd. $\mathrm{m} / \mathrm{z}=274.2[\mathrm{M}+\mathrm{H}]^{+}$ Detected $=274.1[\mathrm{M}+\mathrm{H}]^{+}$

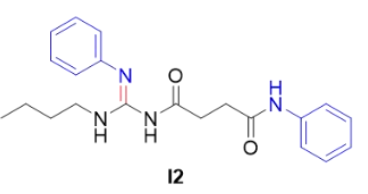

Calcd. $\mathrm{m} / \mathrm{z}=367.2[\mathrm{M}+\mathrm{H}]^{+}$ Detected $=367.1[\mathrm{M}+\mathrm{H}]^{+}$

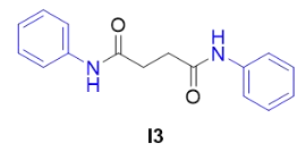

Calcd. $\mathrm{m} / \mathrm{z}=291.1[\mathrm{M}+\mathrm{Na}]$ Detected $=291.2[\mathrm{M}+\mathrm{Na}]^{+}$

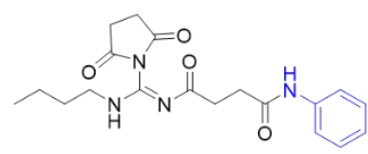

Not detected

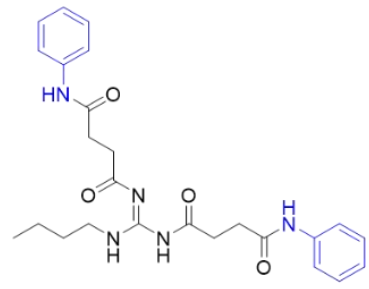

Not detected

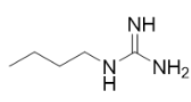

Not detected

Figure S5. LC-MS chromatograms of the crude reaction mixture and detected $\mathrm{m} / \mathrm{z}$ values of possible intermediates during the reaction. 
<smiles>CCCCN=C(N1C(=O)CCC1=O)N1C(=O)CCC1=O</smiles>

$1 \mathrm{a}$

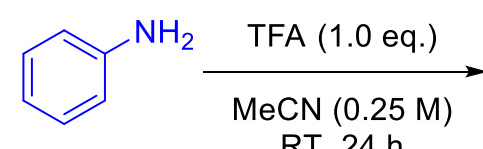

1.0 eq.<smiles>CCCCNC(=Nc1ccccc1)N1C(=O)CCC1=O</smiles>

11<smiles>O=C1CCC(=O)N1c1ccccc1</smiles><smiles>O=C1CCC(=O)N1</smiles>

To a stirred solution of GCDI 1a (139.7 mg, $0.500 \mathrm{mmol}, 1.00$ eq. $)$ and TFA $(0.0392 \mathrm{ml}, 0.500 \mathrm{mmol}$, 1.00 eq.) in $\mathrm{MeCN}(2.00 \mathrm{ml})$, aniline $(0.0457 \mathrm{ml}, 0.500 \mathrm{mmol}, 1.00$ eq.) was added, then the reaction mixture was stirred at ambient temperature. After $24 \mathrm{~h}$ reaction, the reaction mixture was concentrated under reduced pressure. Compound $\mathbf{I 1}$ was isolated as a mixture with $\mathrm{N}$-phenylsuccinimide and succinimide after purification by flash column chromatography $\left(\mathrm{SiO}_{2}\right.$, hexane/ethyl acetate $5: 1 \rightarrow$ ethyl acetate). ${ }^{1} \mathrm{H}$ NMR $\left(500 \mathrm{MHz}, \mathrm{CDCl}_{3}\right): \delta 7.23-7.12(\mathrm{~m}, 2 \mathrm{H}), 7.03-6.91(\mathrm{~m}, 1 \mathrm{H}), 6.76(\mathrm{~d}, J=7.6 \mathrm{~Hz}, 2 \mathrm{H})$, 4.40 (brs, 1H), 3.52-3.38 (m, 2H), 2.50 (brs, 4H), 1.72-1.61 (m, 2H), 1.51-1.37 (m, 2H), $0.96(\mathrm{t}, J=7.2$ $\mathrm{Hz}, 3 \mathrm{H}$ ). Spectral data of $\mathrm{N}$-phenylsuccinimide and succinimide match those previously reported $\mathrm{d}^{5,6}$.

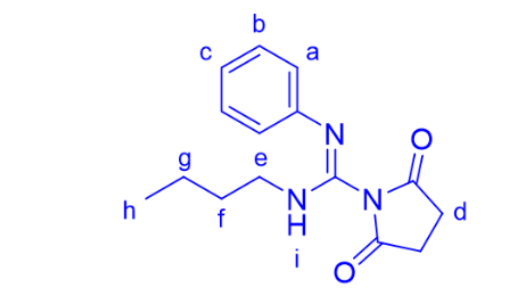<smiles>O=C1CC(Cl)C(=O)N1c1ccccc1</smiles>

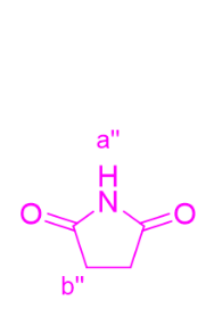
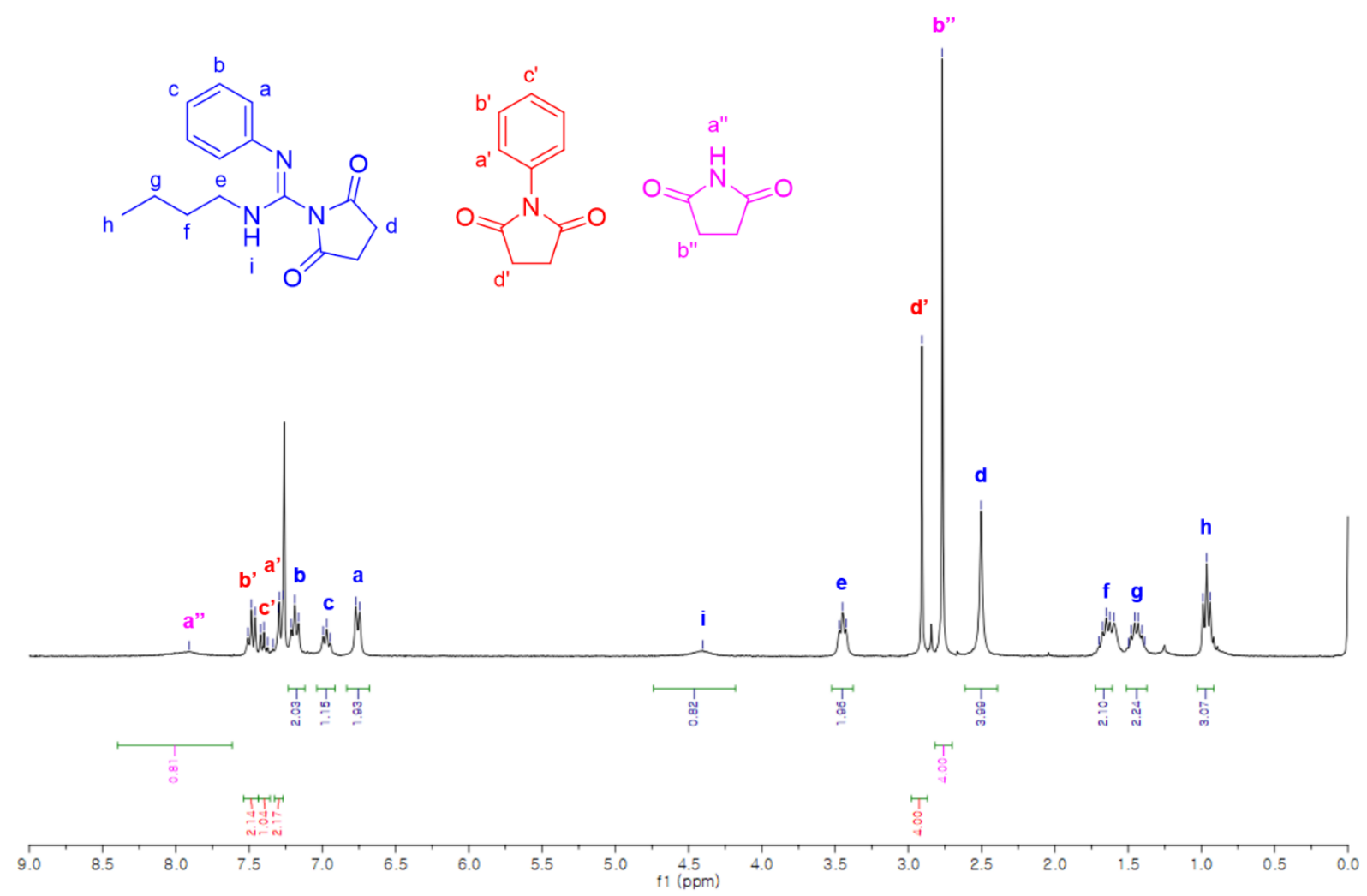

Figure S6. ${ }^{1} \mathrm{H}$ NMR spectra of the mixture of compound I1 with $N$-phenylsuccinimide and succinimide. 
K. LC-MS Analysis of the Reaction with Alkylamine<smiles>CCCCN=C(N1C(=O)CCC1=O)N1C(=O)CCC1=O</smiles>

$1 \mathrm{a}$<smiles>CCCN</smiles>

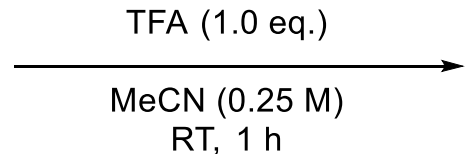

$3.0 \mathrm{eq}$.

To a stirred solution of GCDI 1a (69.8 mg, $0.250 \mathrm{mmol}, 1.00$ eq.) and TFA (0.0196 ml, $0.250 \mathrm{mmol}, 1.00$ eq.) in $\mathrm{MeCN}(1.00 \mathrm{ml})$, propylamine $(0.0629 \mathrm{ml}, 0.750 \mathrm{mmol}, 3.00$ eq.) was added, then the reaction mixture was stirred at ambient temperature. After $1 \mathrm{~h}$ reaction, the reaction mixture was completely solubilized by adding $1.00 \mathrm{ml}$ of DMF. $20 \mu \mathrm{l}$ of the mixture was diluted with $980 \mu \mathrm{l}$ of $\mathrm{H}_{2} \mathrm{O} / \mathrm{MeCN}(\mathrm{v} / \mathrm{v}$ $1: 1)$ solution, then analyzed by ESI/MS-(+) to detect possible reaction intermediates.

\section{Methods for LC-MS Analysis}

LC-MS chromatograms and associated mass spectra were recorded on an Agilent 6120 Quadrupole LCMS System using an ESI-TOF mass spectrometer equipped with a Shiseido UG $1205 \mu \mathrm{m}$ column. Mobile phases are: $0.1 \%$ TFA in water (solvent A) and MeCN (solvent B). Following LC-MS method was used: gradient of $0-10 \mathrm{~min}$ with $5-100 \% \mathrm{~B}$, flow rate: $1.0 \mathrm{ml} / \mathrm{min}$.

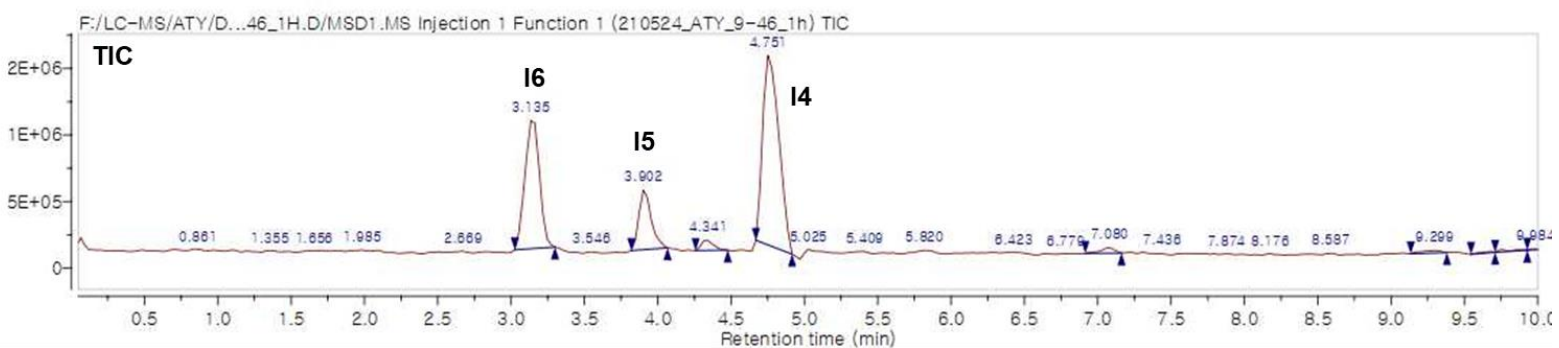

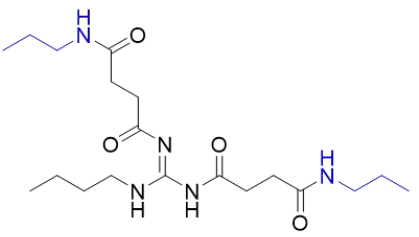

14

Calcd. $\mathrm{m} / \mathrm{z}=398.3[\mathrm{M}+\mathrm{H}]^{+}$ Detected $=398.2[\mathrm{M}+\mathrm{H}]^{+}$

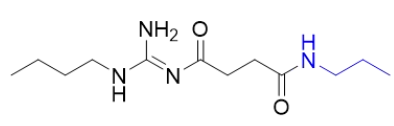

15

Calcd. $\mathrm{m} / \mathrm{z}=257.2[\mathrm{M}+\mathrm{H}]^{+}$ Detected $=257.2[\mathrm{M}+\mathrm{H}]^{+}$

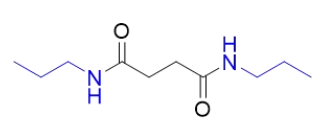

16

Calcd. $\mathrm{m} / \mathrm{z}=201.2[\mathrm{M}+\mathrm{H}]^{+}$ Detected $=201.2[\mathrm{M}+\mathrm{H}]^{+}$

Figure S7. LC-MS chromatograms of the crude reaction mixture and detected $\mathrm{m} / \mathrm{z}$ values of possible intermediates during the reaction. 


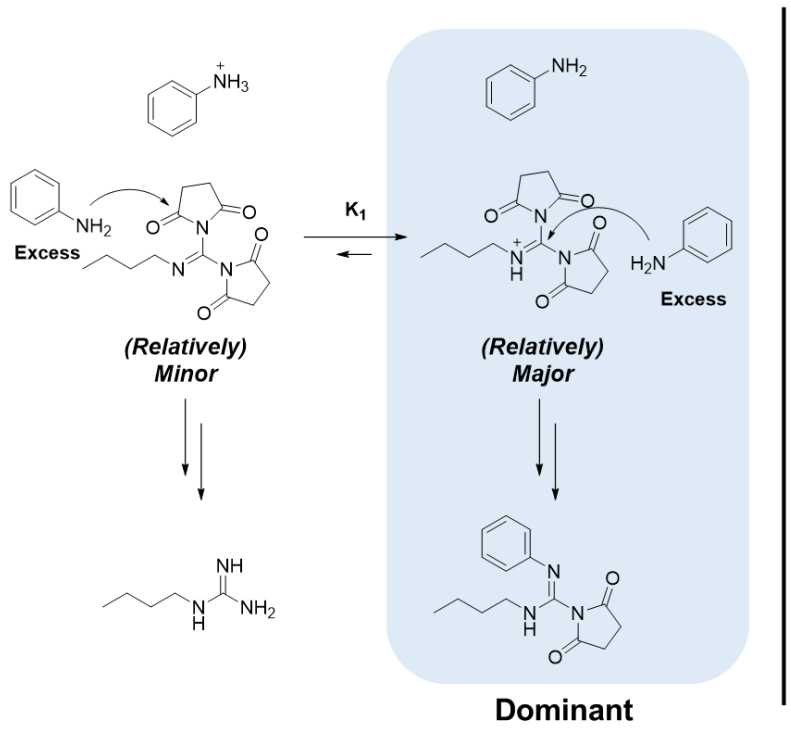

Reactions with aromatic amines $K_{1}=\frac{\left[G C D I H^{+}\right]\left[\mathrm{ArNH}_{2}\right]}{[G C D I]\left[\mathrm{ArNH}_{3}{ }^{*}\right]}=\frac{K_{a}\left(\mathrm{ArNH}_{2}\right)}{K_{a}(G C D I)}$

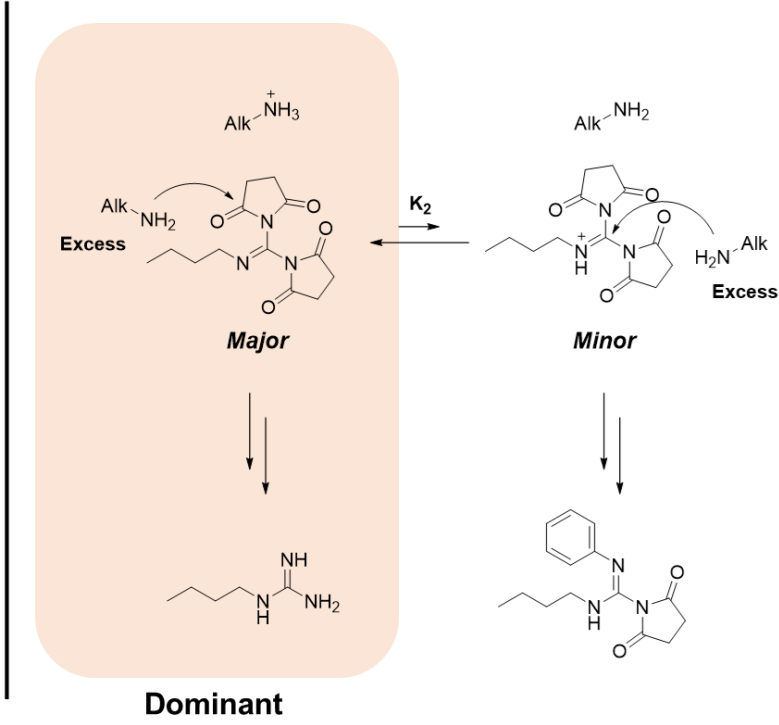

Reactions with aliphatic amines $K_{2}=\frac{\left[G C D I H^{+}\right]\left[\text {AlkNH }_{2}\right]}{[G C D I]\left[\mathrm{AlkNH}_{3}^{+}\right]}=\frac{K_{a}\left(\mathrm{AlkNH}_{2}\right)}{K_{a}(\mathrm{GCDI})}$

$$
\frac{K_{2}}{K_{1}}=\frac{K_{a}\left(A l k N H_{2}\right)}{K_{a}\left(A r N H_{2}\right)}=\sim 10^{-6}
$$

Figure S8. Possible reaction pathways of GCDls with aromatic and aliphatic amines under amineexcess conditions. 
L. A Proposed Mechanism for Formation of $4 a$
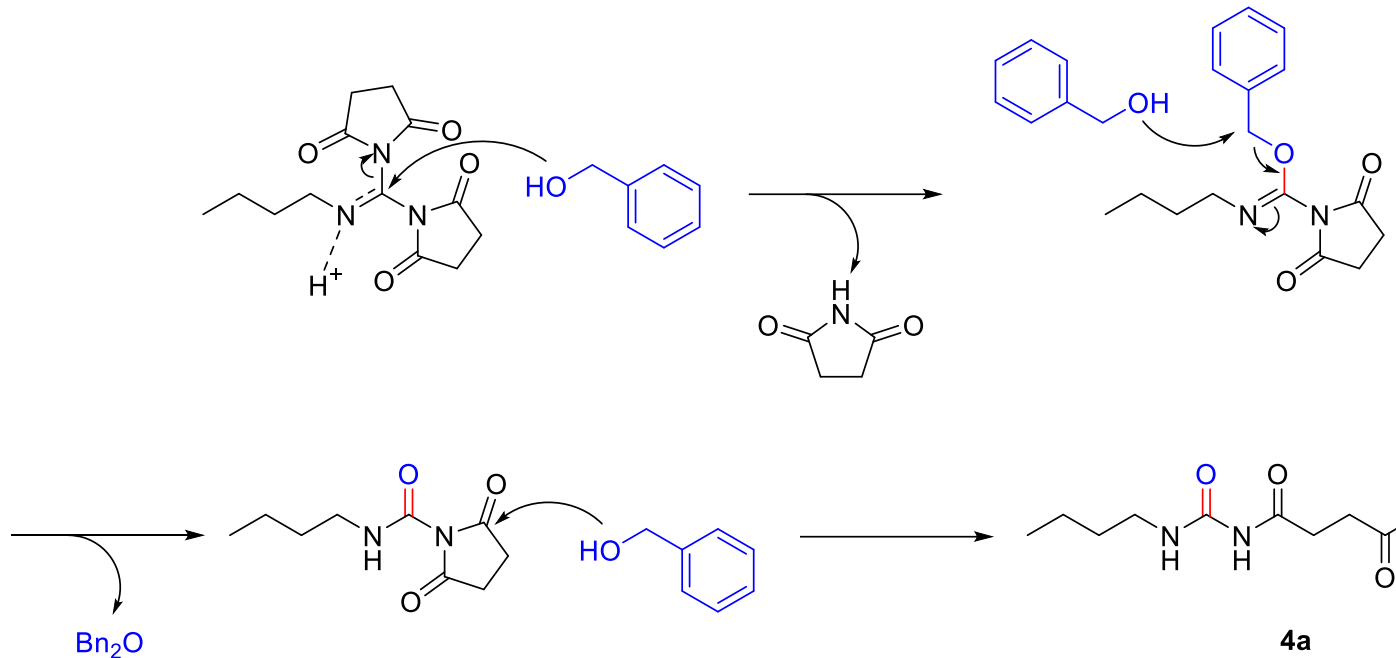<smiles>CCCNC(=O)NC(=O)CCC(=O)OCc1ccccc1</smiles>

$4 a$

Figure S9. A possible mechanism for the reaction of GCDIs with alcohol nucleophiles. 
<smiles></smiles>

1a<smiles>CC(C)CC(C)(C)C(C)(C)C</smiles>

3.0 eq.<smiles>CCCCNC(=O)NC(=O)CCC(=O)OCc1ccccc1</smiles>

$4 a$

To a stirred solution of GCDI 1a (140 mg, $0.500 \mathrm{mmol}, 1.00$ eq.) and TFA in solvent, benzyl alcohol $(0.158 \mathrm{ml}, 1.50 \mathrm{mmol}, 3.00$ eq.) was added, then the reaction mixture was stirred at indicated temperature. After $24 \mathrm{~h}$ reaction, the reaction mixture was concentrated under reduced pressure. The product was purified by flash column chromatography $\left(\mathrm{SiO}_{2}\right.$, hexane $\rightarrow$ hexane/ethyl acetate 1:1).

Table S8. Representative Optimization Results of the Nucleophilic Substitution with Benzyl Alcohol

\begin{tabular}{cccccc}
\hline Entry & Solvent & $\begin{array}{c}\text { eq. of } \\
\text { TFA }\end{array}$ & Temperature & Concentration & Isolated yields \\
\hline $\mathbf{1}$ & MeCN & 1.0 & RT & $0.25 \mathrm{M}$ & $35 \%$ \\
\hline $\mathbf{2}$ & THF & 1.0 & RT & $0.25 \mathrm{M}$ & $31 \%$ \\
\hline $\mathbf{3}$ & DMF & 1.0 & RT & $0.25 \mathrm{M}$ & $12 \%$ \\
\hline $\mathbf{4}$ & Toluene & 1.0 & RT & $0.25 \mathrm{M}$ & $43 \%$ \\
\hline $\mathbf{5}$ & DCM & 1.0 & RT & $0.25 \mathrm{M}$ & $45 \%$ \\
\hline $\mathbf{6}$ & DCM & 2.0 & RT & $0.25 \mathrm{M}$ & $45 \%$ \\
\hline $\mathbf{8}$ & DCM & 1.0 & RT & $0.50 \mathrm{M}$ & $46 \%$ \\
\hline $\mathbf{9}$ & DCM & 1.0 & RT & $0.25 \mathrm{M}$ & $29 \%$ \\
\hline $\mathbf{1 0}$ & Toluene & 1.0 & $70{ }^{\circ} \mathrm{C}$ & $0.25 \mathrm{M}$ & $74 \%$ \\
\hline $\mathbf{1 1}$ & Toluene & 1.0 & $100^{\circ} \mathrm{C}$ & $0.25 \mathrm{M}$ & $68 \%$ \\
\hline $\mathbf{1 2}$ & $1,2-$ Dichloroethane & 1.0 & $70{ }^{\circ} \mathrm{C}$ & $0.25 \mathrm{M}$ & $64 \%$ \\
\hline $\mathbf{1 3}$ & Dioxane & 1.0 & $70{ }^{\circ} \mathrm{C}$ & $0.25 \mathrm{M}$ & $36 \%$ \\
\hline
\end{tabular}

a 1.0 eq. of $\mathrm{H}_{2} \mathrm{O}$ was added to the reaction mixture. 


\section{N. General Procedures for the Nucleophilic Substitution at the Guanidine Carbon Center of GCDIs with Alcohols}

General Procedure C<smiles>[R1]N=C(N1C(=O)CCC1=O)N1C(=O)CCC1=O</smiles>

1<smiles>[R]O</smiles>

3.0 eq.

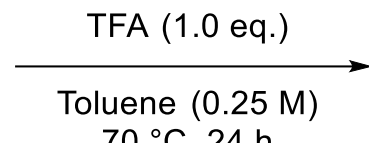

$70{ }^{\circ} \mathrm{C}, 24 \mathrm{~h}$<smiles>[R]NC(=O)NC(=O)CCC(=O)O[R]</smiles>

4

To a stirred solution of GCDI 1 ( $0.500 \mathrm{mmol}, 1.00$ eq.) and TFA $(0.0392 \mathrm{ml}, 0.500 \mathrm{mmol}, 1.00$ eq.) in toluene $(2.00 \mathrm{ml})$, alcohol $(1.50 \mathrm{mmol}, 3.00 \mathrm{eq}$.) was added, then the reaction mixture was placed in an oil bath and stirred at $70{ }^{\circ} \mathrm{C}$. After $24 \mathrm{~h}$ reaction, the reaction mixture was concentrated under reduced pressure. The product was purified by flash column chromatography.

Benzyl 4-(3-butylureido)-4-oxobutanoate (4a)<smiles>CCCCNC(=O)NC(=O)CCC(=O)OCc1ccccc1</smiles>

$4 a$

$\mathrm{N}$-acylurea 4a was afforded (113 $\mathrm{mg}, 74 \%)$ following General Procedure $C$ as a white solid after purification by flash column chromatography $\left(\mathrm{SiO}_{2}\right.$, hexane $\rightarrow$ hexane/ethyl acetate $1: 1) .{ }^{1} \mathrm{H}$ NMR $\left(500 \mathrm{MHz}, \mathrm{CDCl}_{3}\right): \delta$ 10.36 (brs, $1 \mathrm{H}$ ), 8.41 (brs, 1H), 7.41-7.27 (m, 5H), 5.14 (s, 2H), 3.31-3.21 (m, 2H), 2.70 (app s, 4H), 1.56-1.46 $(\mathrm{m}, 2 \mathrm{H}), 1.41-1.30(\mathrm{~m}, 2 \mathrm{H}), 0.92(\mathrm{t}, J=7.3 \mathrm{~Hz}, 3 \mathrm{H}) .{ }^{13} \mathrm{C} \mathrm{NMR}\left(125 \mathrm{MHz}, \mathrm{CDCl}_{3}\right): \delta 173.7,172.2,154.8$, 135.8, 128.6, 128.3, 128.3, 66.7, 39.4, 31.6, 31.2, 28.6, 20.1, 13.8. HRMS (ESI) calculated for $\mathrm{C}_{16} \mathrm{H}_{22} \mathrm{~N}_{2} \mathrm{O}_{4}[\mathrm{M}+\mathrm{H}]^{+}: \mathrm{m} / \mathrm{z} 307.1658$, found 307.1650 .

Octyl 4-(3-butylureido)-4-oxobutanoate (4b)<smiles>CCCCCCCCOC(=O)CCC(=O)NC(=O)NCCCC</smiles>

4b
$\mathrm{N}$-acylurea 4b was afforded (76 $\mathrm{mg}, 47 \%)$ following General Procedure $C$ as a white solid after purification by flash column chromatography $\left(\mathrm{SiO}_{2}\right.$, hexane/ethyl acetate $5: 1 \rightarrow$ hexane/ethyl acetate 2:1). ${ }^{1} \mathrm{H}$ NMR $\left(500 \mathrm{MHz}, \mathrm{CDCl}_{3}\right): \delta 10.33(\mathrm{brs}, 1 \mathrm{H}), 8.40(\mathrm{brs}$,
$56(\mathrm{~m}, 4 \mathrm{H}), 1.65-1.55(\mathrm{~m}, 2 \mathrm{H}), 1.53-1.43(\mathrm{~m}$, $1 \mathrm{H}), 4.06(\mathrm{t}, J=6.8 \mathrm{~Hz}, 2 \mathrm{H}), 3.31-3.18(\mathrm{~m}, 2 \mathrm{H}), 2.72-2.56(\mathrm{~m}, 4 \mathrm{H}), 1.65-1.55(\mathrm{~m}, 2 \mathrm{H}), 1.53-1.43(\mathrm{~m}$,
$2 \mathrm{H}), 1.38-1.18(\mathrm{~m}, 12 \mathrm{H}), 0.89(\mathrm{t}, J=7.3 \mathrm{~Hz}, 3 \mathrm{H}), 0.85(\mathrm{t}, J=6.8 \mathrm{~Hz}, 3 \mathrm{H}) .{ }^{13} \mathrm{C} \mathrm{NMR}\left(125 \mathrm{MHz}, \mathrm{CDCl}_{3}\right)$ : $\delta 173.9,172.5,154.8,65.1,39.4,31.9,31.7,31.2,29.3,29.3,28.6,25.9,22.7,20.1,14.2,13.8$. MS $(E S I)^{+}$calculated for $\mathrm{C}_{17} \mathrm{H}_{32} \mathrm{~N}_{2} \mathrm{O}_{4}[\mathrm{M}+\mathrm{H}]^{+}: \mathrm{m} / \mathrm{z} 329.2$, found 329.2 . 
Pentan-3-yl 4-(3-butylureido)-4-oxobutanoate (4c)<smiles>CCCCNC(=O)NC(=O)CCC(=O)OC(CC)CC</smiles>

4c

$\mathrm{N}$-acylurea 4c was afforded (37 mg, 26\%) following General Procedure $C$ as a colorless solid after purification by flash column chromatography $\left(\mathrm{SiO}_{2}\right.$, hexane/ethyl acetate $5: 1 \rightarrow$ hexane/ethyl acetate 1:1). ${ }^{1} \mathrm{H}$ NMR $\left(500 \mathrm{MHz}, \mathrm{CDCl}_{3}\right): \delta$ 10.13 (brs, $1 \mathrm{H}), 8.40$ (brs, 1H), 4.81-4.72 (m, 1H), 3.30-3.22 $(\mathrm{m}, 2 \mathrm{H}), 2.65$ (app s, 4H), 1.60-1.45 (m, 6H), 1.39-1.29 (m, $2 \mathrm{H}), 0.90(\mathrm{t}, J=7.3 \mathrm{~Hz}, 3 \mathrm{H}), 0.86(\mathrm{t}, J=7.4 \mathrm{~Hz}, 6 \mathrm{H}) .{ }^{13} \mathrm{C} \mathrm{NMR}\left(125 \mathrm{MHz}, \mathrm{CDCl}_{3}\right): \delta 173.9,172.2,154.8$, 77.4, 39.4, 31.7, 31.5, 29.0, 26.5, 20.1, 13.8, 9.7. MS (ESI) calculated for $\mathrm{C}_{14} \mathrm{H}_{26} \mathrm{~N}_{2} \mathrm{O}_{4}[\mathrm{M}+\mathrm{H}]^{+}: \mathrm{m} / \mathrm{z}$ 287.2 , found 287.1 .

tert-Butyl 4-(3-butylureido)-4-oxobutanoate (4d)<smiles>CCCCNC(=O)NC(=O)CCC(=O)OC(C)(C)C</smiles>

$\mathrm{N}$-acylurea $4 \mathrm{~d}$ was afforded $(7 \mathrm{mg}, 5 \%)$ following General Procedure $C$ as a white solid after purification by flash column chromatography $\left(\mathrm{SiO}_{2}\right.$, hexane/ethyl acetate $5: 1 \rightarrow$ hexane/ethyl acetate 1:1). ${ }^{1} \mathrm{H}$ NMR $\left(500 \mathrm{MHz}, \mathrm{CDCl}_{3}\right): \delta 9.48$ (brs, 1H), 8.37 (brs, $1 \mathrm{H}), 3.33-3.23(\mathrm{~m}, 2 \mathrm{H}), 2.63-2.53(\mathrm{~m}, 4 \mathrm{H})$, $1.56-1.48(\mathrm{~m}, 2 \mathrm{H}), 1.44(\mathrm{~s}, 9 \mathrm{H}), 1.40-1.32(\mathrm{~m}, 2 \mathrm{H}), 0.92(\mathrm{t}, J=$ $7.3 \mathrm{~Hz}, 3 \mathrm{H}) .{ }^{13} \mathrm{C} \mathrm{NMR}\left(125 \mathrm{MHz}, \mathrm{CDCl}_{3}\right): \delta 173.8,171.6,154.3,81.1,39.5,31.7,30.0,28.2,20.2,13.9$. MS (ESI)+ calculated for $\mathrm{C}_{13} \mathrm{H}_{24} \mathrm{~N}_{2} \mathrm{O}_{4}[\mathrm{M}+\mathrm{H}]^{+}: \mathrm{m} / \mathrm{z} 273.2$, found 273.1 .

6-Chlorohexyl 4-(3-butylureido)-4-oxobutanoate (4e)<smiles>CCCCNC(=O)NC(=O)CCC(=O)OCCCCCCCl</smiles>

$4 e$

$\mathrm{N}$-acylurea 4e was afforded (96 $\mathrm{mg}, 57 \%$ ) following General Procedure $C$ as a colorless gel after purification by flash column chromatography $\left(\mathrm{SiO}_{2}\right.$, hexane/ethyl acetate $5: 1 \rightarrow$ hexane/ethyl acetate $1: 1) .{ }^{1} \mathrm{H}$ NMR $(500$ $\mathrm{MHz}, \mathrm{CDCl}_{3}$ ): $\delta 10.33$ (brs, $\left.1 \mathrm{H}\right), 8.38$ (brs, $1 \mathrm{H}$ ), $4.06(\mathrm{t}, J=6.6 \mathrm{~Hz}, 2 \mathrm{H}), 3.49(\mathrm{t}, J=6.7 \mathrm{~Hz}, 2 \mathrm{H}), 3.29-3.20(\mathrm{~m}, 2 \mathrm{H}), 2.70-2.56(\mathrm{~m}, 4 \mathrm{H}), 1.78-1.69(\mathrm{~m}$, $2 \mathrm{H}), 1.65-1.56(\mathrm{~m}, 2 \mathrm{H}), 1.52-1.38(\mathrm{~m}, 4 \mathrm{H}), 1.38-1.27(\mathrm{~m}, 4 \mathrm{H}), 0.88(\mathrm{t}, J=7.3 \mathrm{~Hz}, 3 \mathrm{H}) .{ }^{13} \mathrm{C}$ NMR $(125$ $\left.\mathrm{MHz}, \mathrm{CDCl}_{3}\right): \delta 173.8,172.4,154.8,64.7,44.9,39.4,32.5,31.6,31.2,28.6,28.5,26.5,25.2,20.0,13.7$. MS (ESI) ${ }^{+}$calculated for $\mathrm{C}_{15} \mathrm{H}_{27} \mathrm{CIN}_{2} \mathrm{O}_{4}[\mathrm{M}+\mathrm{H}]^{+}: \mathrm{m} / \mathrm{z} 335.2$, found 335.1 .

2-Bromoethyl 4-(3-butylureido)-4-oxobutanoate (4f)<smiles>CCCCNC(=O)NC(=O)CCC(=O)OCCBr</smiles>

4f

$\mathrm{N}$-acylurea $4 \mathbf{f}$ was afforded $(40 \mathrm{mg}, 25 \%)$ following General Procedure $C$ as a colorless solid after purification by flash column chromatography $\left(\mathrm{SiO}_{2}\right.$, hexane/ethyl acetate $5: 1 \rightarrow$ hexane/ethyl acetate $1: 1) .{ }^{1} \mathrm{H}$ NMR (500 $\mathrm{MHz}, \mathrm{CDCl}_{3}$ ): $\delta 10.01$ (brs, $\left.1 \mathrm{H}\right), 8.36$ (brs, $\left.1 \mathrm{H}\right), 4.41$ (t, $J=$ $6.1 \mathrm{~Hz}, 2 \mathrm{H}), 3.50(\mathrm{t}, J=6.1 \mathrm{~Hz}, 2 \mathrm{H}), 3.31-3.23(\mathrm{~m}, 2 \mathrm{H})$, 2.69 (app s, 4H), 1.55-1.47 (m, 2H), 1.40-1.30 (m, 2H), $0.92(\mathrm{t}, J=7.3 \mathrm{~Hz}, 3 \mathrm{H}) .{ }^{13} \mathrm{C}$ NMR $(125 \mathrm{MHz}$, $\left.\mathrm{CDCl}_{3}\right): \delta 173.5,171.9,154.6,64.2,39.5,31.7,31.2,28.7,28.5,20.2,13.9$. MS (ESI)+ calculated for $\mathrm{C}_{11} \mathrm{H}_{19} \mathrm{BrN}_{2} \mathrm{O}_{4}[\mathrm{M}+\mathrm{H}]^{+}: \mathrm{m} / \mathrm{z} 323.1,325.1$. found $323.0,325.1$. 
<smiles>CCCCNC(=O)NC(=O)CCC(=O)OCCI</smiles>

$4 \mathrm{~g}$

$\mathrm{N}$-acylurea $\mathbf{4 g}$ was afforded (56 $\mathrm{mg}, 30 \%$ ) following General Procedure $C$ as a yellow solid after purification by flash column chromatography $\left(\mathrm{SiO}_{2}\right.$, hexane/ethyl acetate $5: 1 \rightarrow$ hexane/ethyl acetate 1:1). ${ }^{1} \mathrm{H}$ NMR $\left(500 \mathrm{MHz}, \mathrm{CDCl}_{3}\right): \delta$ 10.21 (brs, $1 \mathrm{H}), 8.38$ (brs, $1 \mathrm{H}), 4.34(\mathrm{t}, J=6.8 \mathrm{~Hz}, 2 \mathrm{H}), 3.32-$ $3.20(\mathrm{~m}, 4 \mathrm{H}), 2.68($ app s, 4H), 1.55-1.45 (m, 2H), 1.39$1.29(\mathrm{~m}, 2 \mathrm{H}), 0.90(\mathrm{t}, J=7.3 \mathrm{~Hz}, 3 \mathrm{H}) .{ }^{13} \mathrm{C}$ NMR $\left(125 \mathrm{MHz}, \mathrm{CDCl}_{3}\right): \delta 173.6,171.8,154.8,64.9,39.5$, 31.6, 31.1, 28.5, 20.1, 13.8, 0.3. MS (ESI)+ calculated for $\mathrm{C}_{11} \mathrm{H}_{19} \mathrm{IN}_{2} \mathrm{O}_{4}[\mathrm{M}+\mathrm{H}]^{+}: \mathrm{m} / \mathrm{z} 371.0$, found 371.0.

2-Phenoxyethyl 4-(3-butylureido)-4-oxobutanoate (4h)<smiles>CCCCNC(=O)NC(=O)CCC(=O)OCCOc1ccccc1</smiles>

4h

$\mathrm{N}$-acylurea $4 \mathrm{~h}$ was afforded (73 $\mathrm{mg}, 43 \%)$ following General Procedure $C$ as a white solid after purification by flash column chromatography $\left(\mathrm{SiO}_{2}\right.$, hexane/ethyl acetate $5: 1 \rightarrow$ hexane/ethyl acetate 1:1). ${ }^{1} \mathrm{H}$ NMR $\left(500 \mathrm{MHz}, \mathrm{CDCl}_{3}\right): \delta 10.13$ (brs, $\left.1 \mathrm{H}\right)$, 8.36 (brs, $1 \mathrm{H}), 7.33-7.23(\mathrm{~m}, 2 \mathrm{H}), 7.00-6.93(\mathrm{~m}$, $1 \mathrm{H}), 6.90(\mathrm{~d}, J=8.0 \mathrm{~Hz}, 2 \mathrm{H}), 4.50-4.40(\mathrm{~m}, 2 \mathrm{H}), 4.20-4.11(\mathrm{~m}, 2 \mathrm{H}), 3.30-3.20(\mathrm{~m}, 2 \mathrm{H}), 2.69(\operatorname{app~s}$, $4 \mathrm{H}), 1.55-1.44(\mathrm{~m}, 2 \mathrm{H}), 1.40-1.29(\mathrm{~m}, 2 \mathrm{H}), 0.91(\mathrm{t}, J=7.2 \mathrm{~Hz}, 3 \mathrm{H}) .{ }^{13} \mathrm{C}$ NMR $\left(125 \mathrm{MHz}, \mathrm{CDCl}_{3}\right): \delta$ 173.6, 172.4, 158.5, 154.7, 129.6, 121.3, 114.7, 65.8, 63.3, 39.5, 31.6, 31.2, 28.5, 20.1, 13.8. MS (ESI) ${ }^{+}$ calculated for $\mathrm{C}_{17} \mathrm{H}_{24} \mathrm{~N}_{2} \mathrm{O}_{5}[\mathrm{M}+\mathrm{H}]^{+}: \mathrm{m} / \mathrm{z} 337.2$, found 337.1.

But-3-en-1-yl 4-(3-butylureido)-4-oxobutanoate (4i)<smiles>C=CCCOC(=O)CCC(=O)NC(=O)NCCCC</smiles>

$4 \mathbf{i}$

$\mathrm{N}$-acylurea $4 \mathbf{i}$ was afforded (59 $\mathrm{mg}, 44 \%$ ) following General Procedure $C$ as a pale yellow solid after purification by flash column chromatography $\left(\mathrm{SiO}_{2}\right.$, hexane/ethyl acetate $5: 1 \rightarrow$ hexane/ethyl acetate $1: 1) .{ }^{1} \mathrm{H}$ $\operatorname{NMR}\left(500 \mathrm{MHz}, \mathrm{CDCl}_{3}\right): \delta 10.29$ (brs, $\left.1 \mathrm{H}\right), 8.39$ (brs, $\left.1 \mathrm{H}\right)$, $5.84-5.67(\mathrm{~m}, 1 \mathrm{H}), 5.08(\mathrm{~d}, J=17.6 \mathrm{~Hz}, 1 \mathrm{H}), 5.04(\mathrm{~d}, J=$ $10.3 \mathrm{~Hz}, 1 \mathrm{H}), 4.13(\mathrm{t}, J=6.6 \mathrm{~Hz}, 2 \mathrm{H}), 3.33-3.17(\mathrm{~m}, 2 \mathrm{H}), 2.76-2.55(\mathrm{~m}, 4 \mathrm{H}), 2.43-2.29(\mathrm{~m}, 2 \mathrm{H}), 1.57-$ $1.43(\mathrm{~m}, 2 \mathrm{H}), 1.41-1.27(\mathrm{~m}, 2 \mathrm{H}), 0.90(\mathrm{t}, \mathrm{J}=7.3 \mathrm{~Hz}, 3 \mathrm{H}) .{ }^{13} \mathrm{C} \mathrm{NMR}\left(125 \mathrm{MHz}, \mathrm{CDCl}_{3}\right): \delta 173.8,172.3$, $154.8,134.0,117.3,63.9,39.4,33.1,31.6,31.2,28.6,20.1,13.8$. MS (ESI)+ calculated for $\mathrm{C}_{13} \mathrm{H}_{22} \mathrm{~N}_{2} \mathrm{O}_{4}$ $[\mathrm{M}+\mathrm{H}]^{+}: \mathrm{m} / \mathrm{z} 271.2$, found 271.1 .

Prop-2-yn-1-yl 4-(3-butylureido)-4-oxobutanoate (4j)<smiles>C#CCOC(=O)CCC(=O)NC(=O)NCCCC</smiles>

4j

$\mathrm{N}$-acylurea $4 \mathbf{j}$ was afforded (68 $\mathrm{mg}, 54 \%$ ) following General Procedure $C$ as a white solid after purification by flash column chromatography $\left(\mathrm{SiO}_{2}\right.$, hexane/ethyl acetate $5: 1 \rightarrow$ hexane/ethyl acetate 1:1). ${ }^{1} \mathrm{H}$ NMR $\left(500 \mathrm{MHz}, \mathrm{CDCl}_{3}\right): \delta$ 10.42 (brs, $1 \mathrm{H}), 8.37$ (brs, $1 \mathrm{H}), 4.68(\mathrm{~s}, 2 \mathrm{H}), 3.31-3.17(\mathrm{~m}$, $2 \mathrm{H}), 2.67$ (app s, 4H), $2.47(\mathrm{~s}, 1 \mathrm{H}), 1.53-1.43(\mathrm{~m}, 2 \mathrm{H}), 1.39$ $1.27(\mathrm{~m}, 2 \mathrm{H}), 0.89(\mathrm{t}, J=7.3 \mathrm{~Hz}, 3 \mathrm{H}) .{ }^{13} \mathrm{C}$ NMR $\left(125 \mathrm{MHz}, \mathrm{CDCl}_{3}\right): \delta 173.5,171.6,154.8,77.5,75.1$, 52.3, 39.4, 31.6, 31.0, 28.3, 20.1, 13.8. MS (ESI)+ calculated for $\mathrm{C}_{12} \mathrm{H}_{18} \mathrm{~N}_{2} \mathrm{O}_{4}[\mathrm{M}+\mathrm{H}]^{+}: \mathrm{m} / \mathrm{z} 255.1$, found 255.1. 
Phenyl 4-(3-butylureido)-4-oxobutanoate (4k)<smiles>CCCCNC(=O)NC(=O)CCC(=O)Oc1ccccc1</smiles>

4k

$\mathrm{N}$-acylurea $\mathbf{4 k}$ was afforded $(7 \mathrm{mg}, 5 \%)$ following General Procedure $\mathrm{C}$ as a colorless solid after purification by flash column chromatography $\left(\mathrm{SiO}_{2}\right.$, hexane $\rightarrow$ hexane/ethyl acetate 1:1). ${ }^{1} \mathrm{H}$ NMR $\left(500 \mathrm{MHz}, \mathrm{CDCl}_{3}\right): \delta 9.61$ (brs, $\left.1 \mathrm{H}\right)$, 8.37 (brs, 1H), 7.42-7.32 (m, 2H), 7.25-7.19 (m, 1H), 7.09 $(\mathrm{d}, J=7.6 \mathrm{~Hz}, 2 \mathrm{H}), 3.33-3.25(\mathrm{~m}, 2 \mathrm{H}), 2.94-2.86(\mathrm{~m}, 2 \mathrm{H})$, 2.80-2.74 (m, 2H), 1.57-1.49 (m, 2H), 1.40-1.32 (m, 2H), $0.92(\mathrm{t}, J=7.4 \mathrm{~Hz}, 3 \mathrm{H}) .{ }^{13} \mathrm{C}$ NMR $(125 \mathrm{MHz}$, $\left.\mathrm{CDCl}_{3}\right): \delta 173.2,171.0,154.3,150.7,129.6,126.1,121.6,39.6,31.7,31.4,28.9,20.2,13.9$. MS (MALDI) ${ }^{+}$calculated for $\mathrm{C}_{15} \mathrm{H}_{20} \mathrm{~N}_{2} \mathrm{O}_{4}[\mathrm{M}+\mathrm{Na}]^{+}: \mathrm{m} / \mathrm{z} 315.1$, found 315.3.

Benzyl 4-(3-benzylureido)-4-oxobutanoate (4I)<smiles>O=C(CCC(=O)OCc1ccccc1)NC(=O)NCc1ccccc1</smiles>

4I

$\mathrm{N}$-acylurea 4I was afforded (111 $\mathrm{mg}, 65 \%)$ following General Procedure $C$ as a white solid after purification by flash column chromatography $\left(\mathrm{SiO}_{2}\right.$, hexane/ethyl acetate $5: 1 \rightarrow$ hexane/ethyl acetate $2: 1)$. ${ }^{1} \mathrm{H}$ NMR $(500$ $\mathrm{MHz}, \mathrm{CDCl}_{3}$ ): $\delta 10.46$ (brs, $\left.1 \mathrm{H}\right), 8.83$ (brs, $\left.1 \mathrm{H}\right), 7.50-7.12$ $(\mathrm{m}, 10 \mathrm{H}), 5.15(\mathrm{~s}, 2 \mathrm{H}), 4.47(\mathrm{~d}, J=5.5 \mathrm{~Hz}, 2 \mathrm{H}), 2.70(\mathrm{app}$ s, $4 \mathrm{H}) .{ }^{13} \mathrm{C}$ NMR $\left(125 \mathrm{MHz}, \mathrm{CDCl}_{3}\right): \delta 173.8,172.2,154.9$, 138.1, 135.8, 128.7, 128.6, 128.3, 128.3, 127.7, 127.5, 66.7, 43.7, 31.2, 28.6. MS (ESI)+ calculated for $\mathrm{C}_{19} \mathrm{H}_{20} \mathrm{~N}_{2} \mathrm{O}_{4}[\mathrm{M}+\mathrm{H}]^{+}: \mathrm{m} / \mathrm{z} 341.1$, found 341.1 .

Benzyl 4-oxo-4-(3-phenylureido)butanoate (4m)<smiles>O=C(CCC(=O)OCc1ccccc1)NC(=O)Nc1ccccc1</smiles>

$4 \mathrm{~m}$

$\mathrm{N}$-acylurea $4 \mathrm{~m}$ was afforded (77 mg, 47\%) following General Procedure $C$ as a white solid after purification by flash column chromatography $\left(\mathrm{SiO}_{2}\right.$, hexane/ethyl acetate $5: 1 \rightarrow$ hexane/ethyl acetate 2:1). ${ }^{1} \mathrm{H}$ NMR $\left(500 \mathrm{MHz}, \mathrm{CDCl}_{3}\right): \delta$ 10.58 (brs, 1H), 10.27 (brs, $1 \mathrm{H}), 7.52(\mathrm{~d}, J=7.9 \mathrm{~Hz}, 2 \mathrm{H})$, 7.43-7.29 (m, 7H), 7.18-7.09 (m, 1H), $5.18(\mathrm{~s}, 2 \mathrm{H}), 2.80$ (app s, 4H). ${ }^{13} \mathrm{C} \mathrm{NMR}\left(125 \mathrm{MHz}, \mathrm{CDCl}_{3}\right): \delta 174.1,172.1,152.2,137.1,135.7,129.1,128.7,128.4,128.4$, 124.5, 120.4, 66.9, 31.4, 28.6. MS (ESI)+ calculated for $\mathrm{C}_{18} \mathrm{H}_{18} \mathrm{~N}_{2} \mathrm{O}_{4}[\mathrm{M}+\mathrm{H}]^{+}: \mathrm{m} / \mathrm{z} 327.1$, found 327.0.

Benzyl 4-(3-(4-chlorophenyl)ureido)-4-oxobutanoate (4n)<smiles>O=C(CCC(=O)OCc1ccccc1)NC(=O)Nc1ccc(Cl)cc1</smiles>

$4 n$
$\mathrm{N}$-acylurea $4 \mathrm{n}$ was afforded (18 $\mathrm{mg}, 10 \%)$ following General Procedure $C$ as a white solid after purification by flash column chromatography $\left(\mathrm{SiO}_{2}\right.$, hexane/ethyl acetate $5: 1 \rightarrow$ hexane/ethyl acetate $1: 1) .{ }^{1} \mathrm{H}$ NMR $(400$ $\left.\mathrm{MHz}, \mathrm{CDCl}_{3}\right): \delta 10.51$ (brs, $\left.1 \mathrm{H}\right), 9.78$ (brs, $\left.1 \mathrm{H}\right), 7.41(\mathrm{~d}$, $J=8.5 \mathrm{~Hz}, 2 \mathrm{H}), 7.36-7.27(\mathrm{~m}, 5 \mathrm{H}), 7.25(\mathrm{~d}, J=8.6 \mathrm{~Hz}$, $2 \mathrm{H}), 5.13(\mathrm{~s}, 2 \mathrm{H}), 2.73$ (app s, $4 \mathrm{H}) .{ }^{13} \mathrm{C}$ NMR $(100 \mathrm{MHz}$,

$\left.\mathrm{CDCl}_{3}\right): \delta 174.0,172.1,151.8,135.7,135.7,129.2,128.7,128.5,128.4,121.7,67.0,13.5,28.6 . \mathrm{MS}$ $(\mathrm{ESI})^{+}$calculated for $\mathrm{C}_{18} \mathrm{H}_{17} \mathrm{CIN}_{2} \mathrm{O}_{4}[\mathrm{M}+\mathrm{H}]^{+}: \mathrm{m} / \mathrm{z} 361.1$, found 361.1 . 
Benzyl 4-oxo-4-(3-(p-tolyl)ureido)butanoate (4o)<smiles>Cc1ccc(NC(=O)NC(=O)CCC(=O)OCc2ccccc2)cc1</smiles>

$\mathrm{N}$-acylurea 40 was afforded $(66 \mathrm{mg}, 39 \%)$ following General Procedure $C$ as a white solid after purification by flash column chromatography $\left(\mathrm{SiO}_{2}\right.$, hexane/ethyl acetate $5: 1 \rightarrow$ hexane/ethyl acetate $1: 1)$. ${ }^{1} \mathrm{H}$ NMR $(500$ $\left.\mathrm{MHz}, \mathrm{CDCl}_{3}\right): \delta 10.47$ (brs, $\left.1 \mathrm{H}\right), 10.20$ (brs, $\left.1 \mathrm{H}\right), 7.45-$ $7.28(\mathrm{~m}, 7 \mathrm{H}), 7.13(\mathrm{~d}, J=8.1 \mathrm{~Hz}, 2 \mathrm{H}), 5.17(\mathrm{~s}, 2 \mathrm{H}), 2.78$ (app s, 4H), $2.33(\mathrm{~s}, 3 \mathrm{H}) \cdot{ }^{13} \mathrm{C} \mathrm{NMR}\left(125 \mathrm{MHz}, \mathrm{CDCl}_{3}\right)$ : $\delta 174.0,172.1,152.2,135.8,134.5,134.1,129.6,128.7,128.4,128.4,120.5,66.9,31.4,28.7,21.0$. MS (ESI)+ calculated for $\mathrm{C}_{19} \mathrm{H}_{20} \mathrm{~N}_{2} \mathrm{O}_{4}[\mathrm{M}+\mathrm{H}]^{+}: \mathrm{m} / \mathrm{z} 341.1$, found 341.1 .

Benzyl 4-(3-(4-methoxyphenyl)ureido)-4-oxobutanoate (4p)<smiles>COc1ccc(NC(=O)NC(=O)CCC(=O)OCc2ccccc2)cc1</smiles>

$\mathrm{N}$-acylurea $4 \mathrm{p}$ was afforded $(73 \mathrm{mg}, 41 \%)$ following General Procedure $C$ as a white solid after purification by flash column chromatography $\left(\mathrm{SiO}_{2}\right.$, hexane/ethyl acetate $5: 1 \rightarrow$ hexane/ethyl acetate $1: 1)$. ${ }^{1} \mathrm{H} \mathrm{NMR}\left(500 \mathrm{MHz}, \mathrm{CDCl}_{3}\right): \delta 10.40$ (brs, $\left.1 \mathrm{H}\right), 10.18$ (brs, $1 \mathrm{H}), 7.41(\mathrm{~d}, J=8.8 \mathrm{~Hz}, 2 \mathrm{H}), 7.38-7.28(\mathrm{~m}, 5 \mathrm{H})$, $6.87(\mathrm{~d}, J=8.9 \mathrm{~Hz}, 2 \mathrm{H}), 5.17(\mathrm{~s}, 2 \mathrm{H}), 3.79(\mathrm{~s}, 3 \mathrm{H})$, 2.77 (app s, 4H). ${ }^{13} \mathrm{C} \mathrm{NMR}\left(125 \mathrm{MHz}, \mathrm{CDCl}_{3}\right): \delta$ 174.0, 172.1, 156.6, 152.2, 135.8, 130.1, 128.7, 128.4, 128.4, 122.2, 114.3, 66.8, 55.6, 31.4, 28.7. MS (ESI)+ calculated for $\mathrm{C}_{19} \mathrm{H}_{20} \mathrm{~N}_{2} \mathrm{O}_{5}[\mathrm{M}+\mathrm{H}]^{+}: \mathrm{m} / \mathrm{z} 357.1$, found 357.1 . 


\section{O. Single Crystal X-Ray Diffraction (SC-XRD) Analysis of $4 \mathrm{~m}$}

\section{Crystallization of $\mathbf{4 m}$}

$30.0 \mathrm{mg}$ of $4 \mathrm{~m}$ was dissolved in $0.30 \mathrm{ml}$ of ethyl acetate. $1.50 \mathrm{ml}$ of hexane was added, then the mixture was vortexed and filtered. The resulting filtrate was incubated at $4{ }^{\circ} \mathrm{C}$. After overnight incubation, crystal formation was observed.

\section{X-ray Crystallographic Analysis}

The absolute configuration of the compound $4 \mathrm{~m}$ was determined by SC-XRD analysis performed at the Western Seoul Center of Korea Basic Science Institute (KBSI). The data were collected at $223 \mathrm{~K}$ using a Bruker D8 Venture diffractometer equipped with a graphite-monochromated Mo Ka radiation $(\lambda=$ $0.71073 \AA$ ) and a PHOTON 100 CMOS detector. Data collection and integration were carried out with SMART APEX3 and SAINT ${ }^{7}$. Semiempirical absorption corrections based on equivalent reflections were performed using SADABS ${ }^{7}$. The crystal structure was solved by direct methods and refined by full-matrix least-squares refinement using SHELXTL8.

CCDC 2069064 contains the supplementary crystallographic data for this paper. This data can be obtained free of charge via www.ccdc.cam.ac.uk/conts/retrieving.html (or from the Cambridge Crystallographic Data Centre, 12 Union Road, Cambridge CB21EZ, UK; fax: (+44)1223-336-033; or deposit@ccdc.cam.ac.uk).

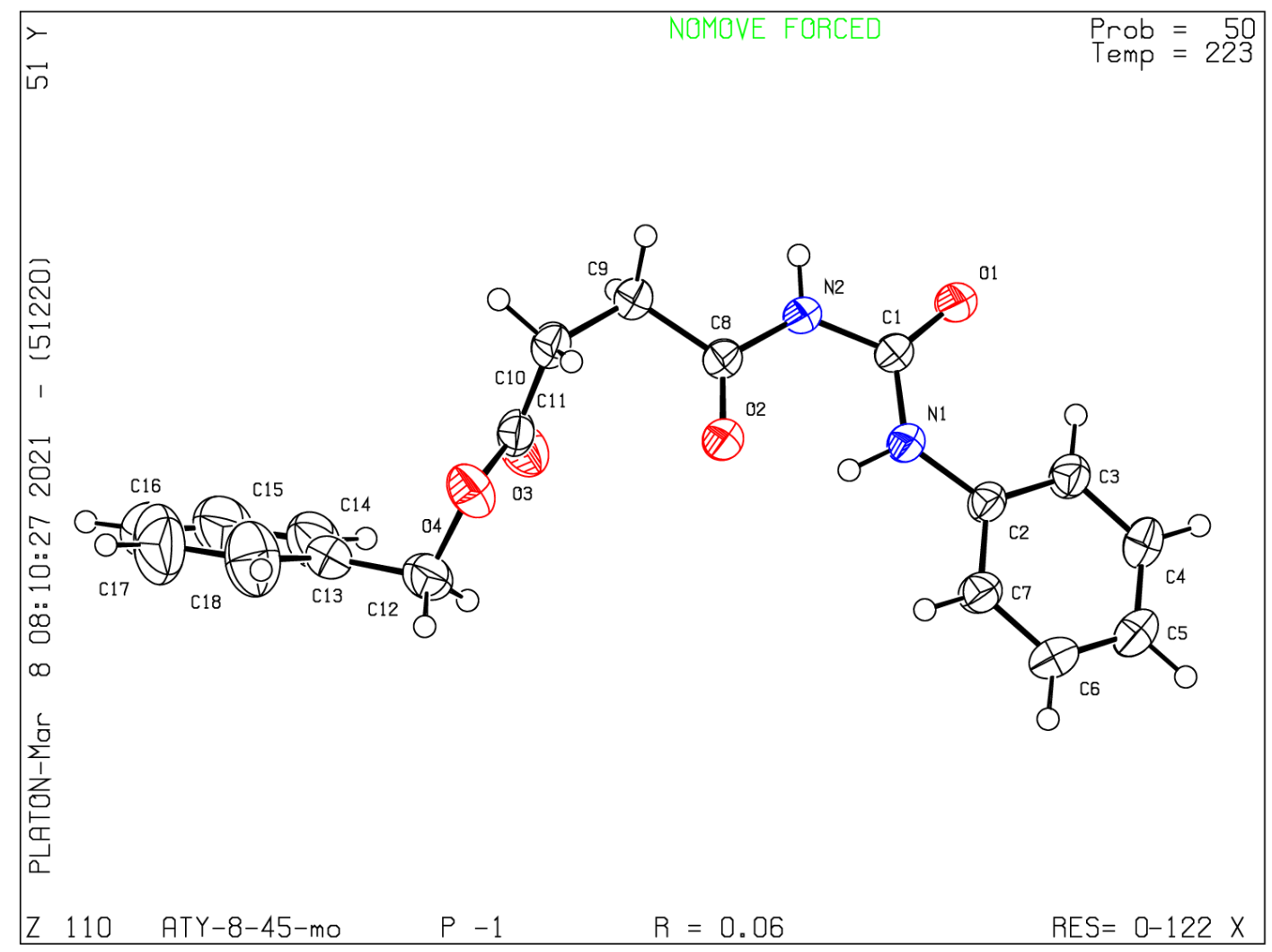

Figure S10. The X-ray crystal structure of $4 \mathrm{~m}$. Thermal ellipsoids represent $50 \%$ probability. 
Table S9. Crystal Structure and Structure Refinement for $4 \mathrm{~m}$

\begin{tabular}{|c|c|c|}
\hline Identification code & \multicolumn{2}{|l|}{ ATY-8-45-mo } \\
\hline Empirical formula & \multicolumn{2}{|l|}{$\mathrm{C} 18 \mathrm{H} 18 \mathrm{~N} 2 \mathrm{O} 4$} \\
\hline Formula weight & \multicolumn{2}{|l|}{326.34} \\
\hline Temperature & \multicolumn{2}{|l|}{ 223(2) K } \\
\hline Wavelength & \multicolumn{2}{|l|}{$0.71073 \AA$} \\
\hline Crystal system & \multicolumn{2}{|l|}{ Triclinic } \\
\hline Space group & \multicolumn{2}{|l|}{$\mathrm{P}-1$} \\
\hline \multirow[t]{3}{*}{ Unit cell dimensions } & $a=5.8013(11) \AA$ & $\alpha=81.253(9)^{\circ}$ \\
\hline & $b=9.2851(18) \AA$ & $\beta=89.499(9)^{\circ}$ \\
\hline & $c=15.809(3) \AA$ & $\chi=80.704(6)^{\circ}$ \\
\hline Volume & \multicolumn{2}{|l|}{$830.5(3) \AA^{3}$} \\
\hline Z & \multicolumn{2}{|l|}{2} \\
\hline Density (calculated) & \multicolumn{2}{|l|}{$1.305 \mathrm{Mg} / \mathrm{m}^{3}$} \\
\hline Absorption coefficient & \multicolumn{2}{|l|}{$0.093 \mathrm{~mm}^{-1}$} \\
\hline$F(000)$ & \multicolumn{2}{|l|}{344} \\
\hline Crystal size & \multicolumn{2}{|c|}{$0.320 \times 0.060 \times 0.033 \mathrm{~mm}^{3}$} \\
\hline Theta range for data collection & \multicolumn{2}{|l|}{2.249 to $28.751^{\circ}$} \\
\hline Index ranges & \multicolumn{2}{|c|}{$-7<=h<=7,-12<=k<=12,-21<=\mid<=21$} \\
\hline Reflections collected & \multicolumn{2}{|l|}{25386} \\
\hline Independent reflections & \multicolumn{2}{|c|}{$4255[R($ int $)=0.0579]$} \\
\hline Completeness to theta $=25.242^{\circ}$ & \multicolumn{2}{|l|}{$99.8 \%$} \\
\hline Absorption correction & \multicolumn{2}{|c|}{ Semi-empirical from equivalents } \\
\hline Max. and min. transmission & \multicolumn{2}{|l|}{0.7457 and 0.6715} \\
\hline Refinement method & \multicolumn{2}{|c|}{ Full-matrix least-squares on $\mathrm{F}^{2}$} \\
\hline Data / restraints / parameters & \multicolumn{2}{|l|}{4255 / 0 / 225} \\
\hline Goodness-of-fit on $\mathrm{F}^{2}$ & \multicolumn{2}{|l|}{1.029} \\
\hline Final R indices [l>2sigma(I)] & \multicolumn{2}{|c|}{$\mathrm{R} 1=0.0560, \mathrm{wR} 2=0.1426$} \\
\hline$R$ indices (all data) & \multicolumn{2}{|c|}{$\mathrm{R} 1=0.0997, \mathrm{wR} 2=0.1713$} \\
\hline Extinction coefficient & \multicolumn{2}{|l|}{$\mathrm{n} / \mathrm{a}$} \\
\hline Largest diff. peak and hole & \multicolumn{2}{|c|}{0.306 and -0.240 e. $\AA^{-3}$} \\
\hline
\end{tabular}




\section{P. Synthesis of 2-Aminobenzimidazole 5}<smiles>CCCCN=C(N1C(=O)CCC1=O)N1C(=O)CCC1=O</smiles>

$1 \mathrm{a}$<smiles>Nc1ccccc1N</smiles>

3.0 eq.

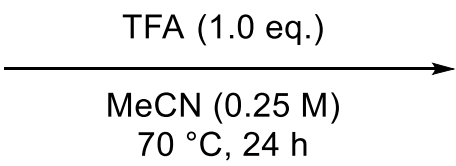

$70{ }^{\circ} \mathrm{C}, 24 \mathrm{~h}$<smiles>CCCCNc1nc2ccccc2[nH]1</smiles>

$5,31 \%$

To a stirred solution of GCDI 1a (140 mg, $0.500 \mathrm{mmol}, 1.00$ eq.) and TFA ( $0.0392 \mathrm{ml}, 0.500 \mathrm{mmol}, 1.00$ eq.) in $\mathrm{MeCN}$ (2.00 ml), o-phenylenediamine (162 mg, $1.50 \mathrm{mmol}, 3.00$ eq.) was added, then the reaction mixture was placed in an oil bath and stirred at $70^{\circ} \mathrm{C}$. After $24 \mathrm{~h}$ reaction, the reaction mixture was concentrated under reduced pressure. 2-Aminobenzimidazole 5 was afforded $(29 \mathrm{mg}, 31 \%)$ as a yellow solid after purification by flash column chromatography $\left(\mathrm{SiO}_{2}\right.$, hexane/ethyl acetate $5: 1 \rightarrow$ hexane/ethyl acetate 1:3). ${ }^{1} \mathrm{H}$ NMR $\left(500 \mathrm{MHz}, \mathrm{CDCl}_{3}\right): \delta 7.28(\mathrm{dd}, J=5.3,3.3 \mathrm{~Hz}, 2 \mathrm{H}), 7.03(\mathrm{dd}, J=$ $5.5,3.1 \mathrm{~Hz}, 2 \mathrm{H}), 5.26(\mathrm{brs}, 1 \mathrm{H}), 3.46-3.37(\mathrm{~m}, 2 \mathrm{H}), 1.63-1.49(\mathrm{~m}, 2 \mathrm{H}), 1.32-1.24(\mathrm{~m}, 2 \mathrm{H}), 0.83(\mathrm{t}, J=$ $7.3 \mathrm{~Hz}, 3 \mathrm{H}) .{ }^{13} \mathrm{C}$ NMR $\left(125 \mathrm{MHz}, \mathrm{CDCl}_{3}\right): \delta 155.4,136.4,121.1,111.9,43.2,32.0,20.0,13.7 . \mathrm{MS}(\mathrm{ESI})^{+}$ calculated for $\mathrm{C}_{11} \mathrm{H}_{15} \mathrm{~N}_{3}[\mathrm{M}+\mathrm{H}]^{+}: \mathrm{m} / \mathrm{z} 190.1$, found 190.1. Spectral data match those previously reported ${ }^{9}$. 


\section{Q. Synthesis of 2-Aminobenzoxazole 6}<smiles>CCCCN=C(N1C(=O)CCC1=O)N1C(=O)CCC1=O</smiles>

$1 \mathrm{a}$<smiles>Nc1ccccc1O</smiles>

$3.0 \mathrm{eq}$

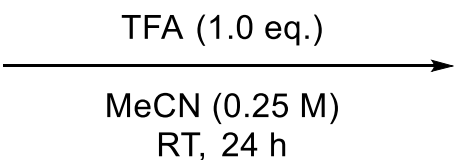

$\mathrm{RT}, 24 \mathrm{~h}$<smiles>CCCCNc1nc2ccccc2o1</smiles>

6, $71 \%$

To a stirred solution of GCDI 1a (140 mg, $0.500 \mathrm{mmol}, 1.00$ eq.) and TFA ( $0.0392 \mathrm{ml}, 0.500 \mathrm{mmol}, 1.00$ eq.) in $\mathrm{MeCN}(2.00 \mathrm{ml}), 2$-aminophenol $(164 \mathrm{mg}, 1.50 \mathrm{mmol}, 3.00$ eq.) was added, then the reaction mixture was stirred at ambient temperature. After $24 \mathrm{~h}$ reaction, the reaction mixture was concentrated under reduced pressure. 2-Aminobenzoxazole 6 was afforded (68 $\mathrm{mg}, 71 \%$ ) as a brown solid after purification by flash column chromatography $\left(\mathrm{SiO}_{2}\right.$, hexane $\rightarrow$ hexane/ethyl acetate $\left.5: 1\right)$. ${ }^{1} \mathrm{H}$ NMR (500 $\mathrm{MHz}, \mathrm{CDCl}_{3}$ ): $\delta 8.05$ (brs, $\left.1 \mathrm{H}\right), 7.33(\mathrm{~d}, J=7.8 \mathrm{~Hz}, 1 \mathrm{H}), 7.28(\mathrm{~d}, J=8.0 \mathrm{~Hz}, 1 \mathrm{H}), 7.23-7.16(\mathrm{~m}, 1 \mathrm{H})$, 7.12-7.04 (m, 1H), $3.49(\mathrm{t}, J=7.2 \mathrm{~Hz}, 2 \mathrm{H}), 1.73-1.62(\mathrm{~m}, 2 \mathrm{H}), 1.48-1.37(\mathrm{~m}, 2 \mathrm{H}), 0.95(\mathrm{t}, J=7.4 \mathrm{~Hz}$, $3 \mathrm{H}) .{ }^{13} \mathrm{C}$ NMR $\left(125 \mathrm{MHz}, \mathrm{CDCl}_{3}\right): \delta 161.2,147.2,137.9,124.9,121.9,114.8,109.5,42.9,31.7,20.0$, 13.7. MS (ESI) calculated for $\mathrm{C}_{11} \mathrm{H}_{14} \mathrm{~N}_{2} \mathrm{O}[\mathrm{M}+\mathrm{H}]^{+}: \mathrm{m} / \mathrm{z}$ 191.1, found 191.1. Spectral data match those previously reported ${ }^{10}$. 


\section{R. Synthesis of Sigma1 Inhibitor 7 (CT-189)}

\section{Preparation of the GCDI precursor $1 \mathrm{k}$}
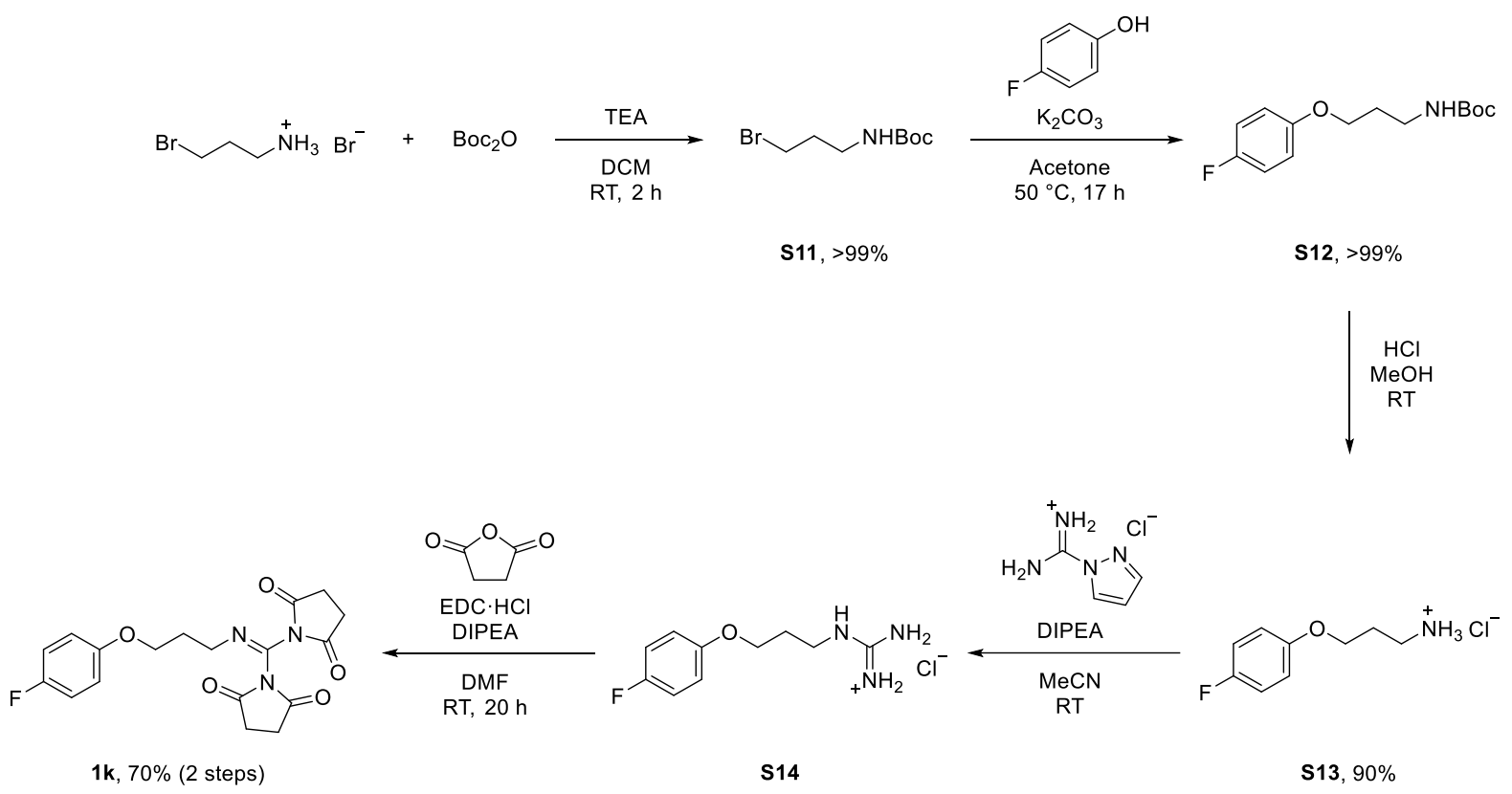

tert-Butyl (3-bromopropyl)carbamate (S11)

$\mathrm{Br} N \mathrm{NHBoc}$ To a stirred solution of 3-bromopropylamine hydrobromide $(4.60 \mathrm{~g}, 21.0 \mathrm{mmol}$, 1.05 eq.) and $\mathrm{Boc}_{2} \mathrm{O}$ (4.37 g, $20.0 \mathrm{mmol}, 1.00$ eq.) in DCM (100 ml), TEA (2.93

$\mathbf{s} 11 \mathrm{ml}, 21.0 \mathrm{mmol}, 1.05$ eq.) was added. The reaction mixture was stirred at ambient temperature for $2 \mathrm{~h}$, then concentrated under reduced pressure. The crude mixture was diluted with ethyl acetate and washed with a saturated aqueous $\mathrm{NH}_{4} \mathrm{Cl}$ solution. The organic layer was further washed with brine. The combined organic layers were dried over anhydrous $\mathrm{MgSO}_{4}$, filtered, then concentrated under reduced pressure to afford $\mathbf{S} 11(4.96 \mathrm{~g},>99 \%)$. The product was used without further purification. ${ }^{1} \mathrm{H}$ NMR $\left(500 \mathrm{MHz}, \mathrm{CDCl}_{3}\right): \delta 4.65$ (brs, $\left.1 \mathrm{H}\right), 3.44$ (t, $J=6.5 \mathrm{~Hz}$, $2 \mathrm{H}), 3.31-3.23(\mathrm{~m}, 2 \mathrm{H}), 2.09-2.00(\mathrm{~m}, 2 \mathrm{H}), 1.44(\mathrm{~s}, 9 \mathrm{H})$. Spectral data match those previously reported ${ }^{11}$.

tert-Butyl (3-(4-fluorophenoxy)propyl)carbamate (S12) (ATY 9-9-2)<smiles>CCOC(=O)NCCCOc1ccc(F)cc1</smiles>

S12

To a stirred solution of $\mathbf{S} 11$ (4.96 g, $20.0 \mathrm{mmol}, 1.00 \mathrm{eq}$.) and 4fluorophenol $\left(6.73 \mathrm{~g}, 60.0 \mathrm{mmol}, 3.00\right.$ eq.) in acetone $(40 \mathrm{ml}), \mathrm{K}_{2} \mathrm{CO}_{3}$ $(5.53 \mathrm{~g}, 40.0 \mathrm{mmol}, 2.00 \mathrm{eq}$.) was added. The reaction mixture was placed in an oil bath and stirred at $50{ }^{\circ} \mathrm{C}$ for $17 \mathrm{~h}$, then concentrated under reduced pressure. The crude mixture was diluted with ethyl acetate and washed with a $2 \mathrm{M}$ aqueous $\mathrm{NaOH}$ solution. The organic layer was further washed with $2 \mathrm{M}$ aqueous $\mathrm{NaOH}$ solution and brine. The combined organic layers were dried over anhydrous $\mathrm{MgSO}_{4}$, filtered, then concentrated under reduced pressure. $\mathbf{S 1 2}$ was afforded $(5.44 \mathrm{~g},>99 \%)$ as a pale yellow solid after purification by flash column chromatography $\left(\mathrm{SiO}_{2}\right.$, hexane $\rightarrow$ hexane/ethyl acetate 4:1). ${ }^{1} \mathrm{H}$ NMR $\left(500 \mathrm{MHz}, \mathrm{CDCl}_{3}\right): \delta 6.98-6.89(\mathrm{~m}, 2 \mathrm{H}), 6.84-6.75(\mathrm{~m}$, 2H), 4.83 (brs, 1H), $3.95(\mathrm{t}, J=5.7 \mathrm{~Hz}, 2 \mathrm{H}), 3.36-3.20(\mathrm{~m}, 2 \mathrm{H}), 1.99-1.87(\mathrm{~m}, 2 \mathrm{H}), 1.42(\mathrm{~s}, 9 \mathrm{H}) .{ }^{13} \mathrm{C}$ 
NMR $\left(125 \mathrm{MHz}, \mathrm{CDCl}_{3}\right): \delta 158.3,156.4,156.1,155.0,155.0,115.9,115.8,115.5,115.5,79.3,66.5$, 38.1, 29.7, 28.5. MS (ESI)+ calculated for $\mathrm{C}_{14} \mathrm{H}_{20} \mathrm{FNO}_{3}[\mathrm{M}+\mathrm{Na}]^{+}: \mathrm{m} / \mathrm{z} 292.1$, found 292.1 .

3-(4-Fluorophenoxy)propan-1-amine hydrochloride (S13)<smiles>[NH3+]CCCOc1ccc(F)cc1</smiles>

S13

S12 $(5.44 \mathrm{~g}, 20.0 \mathrm{mmol})$ was dissolved in a $0.5 \mathrm{M} \mathrm{HCl}$ solution in $\mathrm{MeOH}(120 \mathrm{ml})$, then the reaction mixture was stirred at ambient temperature for overnight. The crude mixture was concentrated under reduced pressure, diluted with minimal amount of $\mathrm{MeOH}$, then excess amount of diethyl ether was added. The resulting precipitate was collected, further washed with diethyl ether, then dried in vacuo to afford S13 (3.71 g, 90\%) as a white solid. ${ }^{1} \mathrm{H}$ NMR (500 MHz, DMSO-d6): $\delta 8.27$ (brs, 3H), 7.15-7.06 $(\mathrm{m}, 2 \mathrm{H}), 6.99-6.90(\mathrm{~m}, 2 \mathrm{H}), 4.03(\mathrm{t}, J=5.6 \mathrm{~Hz}, 2 \mathrm{H}), 2.92(\mathrm{t}, J=7.1 \mathrm{~Hz}, 2 \mathrm{H}), 2.09-1.98(\mathrm{~m}, 2 \mathrm{H}) .{ }^{13} \mathrm{C}$ NMR (125 MHz, DMSO-d6): $\delta$ 157.5, 155.6, 154.7, 154.7, 115.9, 115.8, 115.7, 115.7, 65.2, 36.1, 26.8. MS $(\mathrm{ESI})^{+}$calculated for $\mathrm{C}_{9} \mathrm{H}_{12} \mathrm{FNO}[\mathrm{M}+\mathrm{H}]^{+}: \mathrm{m} / \mathrm{z}$ 170.1, found 170.1 .

1-(3-(4-Fluorophenoxy)propyl)guanidine hydrochloride (S14)<smiles>NC(=[NH2+])NCCCOc1ccc(F)cc1</smiles>

S14

To a stirred solution of $\mathbf{S} 13$ (823 $\mathrm{mg}, 4.00 \mathrm{mmol}, 1.00$ eq.), 1 amidinopyrazole hydrochloride (586 mg, $4.00 \mathrm{mmol}, 1.00 \mathrm{mmol}$ ) in $\operatorname{MeCN}(8.00 \mathrm{ml})$, DIPEA $(1.43 \mathrm{ml}, 8.40 \mathrm{mmol}, 2.10$ eq.) was added. The reaction mixture was stirred at ambient temperature for overnight, then concentrated under reduced pressure. The crude mixture was diluted with minimal amount of $\mathrm{MeOH}$, then excess amount of diethyl ether was added. The resulting precipitate was collected, further washed with diethyl ether, then dried in vacuo to afford S14 as a mixture with $\mathrm{N}, \mathrm{N}$ diisopropylethylamine hydrochloride salt. The product was used without further purification. ${ }^{1} \mathrm{H}$ NMR (500 MHz, CD $\left.{ }_{3} \mathrm{OD}\right): \delta 7.05-6.98(\mathrm{~m}, 2 \mathrm{H}), 6.98-6.91(\mathrm{~m}, 2 \mathrm{H}), 4.05(\mathrm{t}, J=6.0 \mathrm{~Hz}, 2 \mathrm{H}), 3.42(\mathrm{t}, J=6.8$ $\mathrm{Hz}, 2 \mathrm{H}), 2.10-2.00$ (m, 2H). ${ }^{13} \mathrm{C}$ NMR (125 MHz, CD $\left.{ }_{3} \mathrm{OD}\right): \delta$ 159.5, 158.7, 158.6, 157.6, 156.4, 156.3, 116.9, 116.8, 116.8, 116.6, 66.7, 39.5, 29.7. $\mathrm{MS}(\mathrm{ESI})^{+}$calculated for $\mathrm{C}_{10} \mathrm{H}_{15} \mathrm{FN}_{3} \mathrm{O}^{+}[\mathrm{M}+\mathrm{H}]^{+}: \mathrm{m} / \mathrm{z} 212.1$, found 212.1 .

1,1'-(((3-(4-Fluorophenoxy)propyl)imino)methylene)bis(pyrrolidine-2,5-dione) (1k)<smiles>O=C1CCC(=O)N1C(=NCCCOc1ccc(F)cc1)N1C(=O)CCC1=O</smiles>

$1 \mathrm{k}$

To a stirred solution of $\mathbf{S 1 4}$ from the previous step, succinic anhydride $(1.20 \mathrm{~g}, 12.0 \mathrm{mmol}, 3.00$ eq. $)$ and $\mathrm{EDC} \cdot \mathrm{HCl}(1.53 \mathrm{~g}$, $8.00 \mathrm{mmol}, 2.00$ eq.) in DMF (4.00 ml), DIPEA ( $3.40 \mathrm{ml}, 20.0 \mathrm{mmol}$, 5.00 eq.) was added. The reaction mixture was stirred at ambient temperature for $20 \mathrm{~h}$, then concentrated under reduced pressure. The crude mixture was diluted with ethyl acetate and washed with a $0.01 \mathrm{M}$ aqueous $\mathrm{HCl}$. The organic layer was further washed with brine. The combined organic layers were dried over anhydrous $\mathrm{MgSO}_{4}$, filtered, then concentrated under reduced pressure. The product was purified by flash column chromatography. $1 \mathbf{k}$ was afforded ( $1.05 \mathrm{~g}, 70 \%$ over 2 steps) as a pale yellow solid after purification by flash column chromatography $\left(\mathrm{SiO}_{2}\right.$, hexane/ethyl acetate $2: 1 \rightarrow$ hexane/ethyl acetate $\left.1: 3\right) .{ }^{1} \mathrm{H}$ NMR (500 MHz, $\left.\mathrm{CDCl}_{3}\right): \delta 6.92-6.81(\mathrm{~m}, 2 \mathrm{H}), 6.77-6.69(\mathrm{~m}, 2 \mathrm{H}), 3.93(\mathrm{t}, J=5.2 \mathrm{~Hz}, 2 \mathrm{H}), 3.55(\mathrm{t}, J=5.6 \mathrm{~Hz}$, $2 \mathrm{H}$ ), 2.64 (app s, 8H), 2.10-2.00 (m, 2H). $\left.{ }^{13} \mathrm{C} \mathrm{NMR} \mathrm{(125} \mathrm{MHz,} \mathrm{CDCl}_{3}\right): \delta 173.9,173.4,157.9,156.0$, 154.9, 129.1, 115.6, 115.4, 115.4, 65.3, 47.3, 29.3, 28.1, 27.9. MS (ESI)+ calculated for $\mathrm{C}_{18} \mathrm{H}_{18} \mathrm{FN}_{3} \mathrm{O}_{5}$ $[\mathrm{M}+\mathrm{H}]^{+}: \mathrm{m} / \mathrm{z} 376.1$, found 376.1 . 
Synthesis of 7 (CT-189)<smiles>Nc1ccc(Cl)cc1</smiles>

$1 \mathrm{k}$

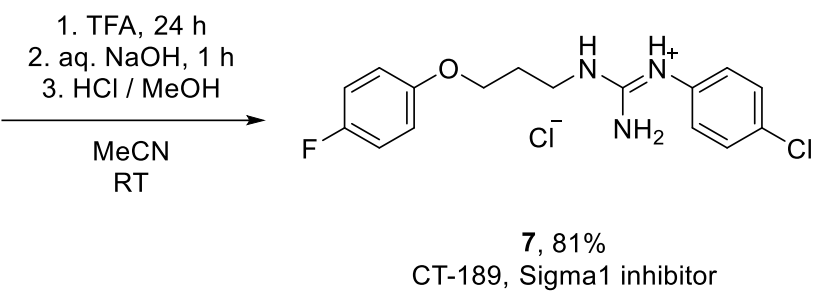

CT-189, Sigma1 inhibito

Following General Procedure A, 7 was synthesized as follows:

To a stirred solution of $\mathbf{1 k}(1.05 \mathrm{~g}, 2.79 \mathrm{mmol}, 1.00$ eq.) and TFA $(0.218 \mathrm{ml}, 2.79 \mathrm{mmol}, 1.00 \mathrm{eq}$.$) in$ MeCN (11.1 ml), 4-chloroaniline $(1.07 \mathrm{~g}, 8.36 \mathrm{mmol}, 3.00 \mathrm{eq}$.) was added, then the reaction mixture was stirred at ambient temperature. After $24 \mathrm{~h}$ reaction, the mixture was quenched by the addition of a $0.5 \mathrm{M}$ aqueous $\mathrm{NaOH}$ solution $(50 \mathrm{ml})$ and further stirred for $30 \mathrm{~min}$. The reaction mixture was concentrated under reduced pressure, diluted with ethyl acetate, then filtered. Subsequently, a $0.5 \mathrm{M}$ $\mathrm{HCl}$ solution in $\mathrm{MeOH}(50 \mathrm{ml})$ was added to the filtrate and the mixture was concentrated under reduced pressure. The residue was dissolved in DCM, then filtered. Sigma 1 inhibitor 7 was afforded $(806 \mathrm{mg}$, $81 \%)$ as a pale yellow solid after purification by flash column chromatography $\left(\mathrm{SiO}_{2}, \mathrm{DCM} \rightarrow\right.$ DCM/MeOH 9:1). ${ }^{1} \mathrm{H}$ NMR (500 MHz, $\left.\mathrm{CD}_{3} \mathrm{OD}\right): \delta 7.43(\mathrm{~d}, J=8.6 \mathrm{~Hz}, 2 \mathrm{H}), 7.25(\mathrm{~d}, J=8.6 \mathrm{~Hz}, 2 \mathrm{H})$, 7.05-6.96 (m, 2H), 6.96-6.88 (m, 2H), $4.08(\mathrm{t}, J=5.8 \mathrm{~Hz}, 2 \mathrm{H}), 3.54(\mathrm{t}, J=6.6 \mathrm{~Hz}, 2 \mathrm{H}), 2.16-2.07(\mathrm{~m}$, 2H). ${ }^{13} \mathrm{C}$ NMR $\left(125 \mathrm{MHz}, \mathrm{CD}_{3} \mathrm{OD}\right): \delta 159.6,157.7,156.8,156.3,156.3,135.1,133.8,131.0,127.9$, 116.8, 116.8, 116.7, 116.6, 66.9, 40.3, 29.5. MS (ESI)+ calculated for $\mathrm{C}_{16} \mathrm{H}_{17} \mathrm{ClFN}_{3} \mathrm{O}[\mathrm{M}+\mathrm{H}]^{+}: \mathrm{m} / \mathrm{z} 322.1$, found 322.1. 


\section{S. Synthesis of $\alpha$-Glucosidase Inhibitor 8}

\section{Preparation of the GCDI precursor 1 I}

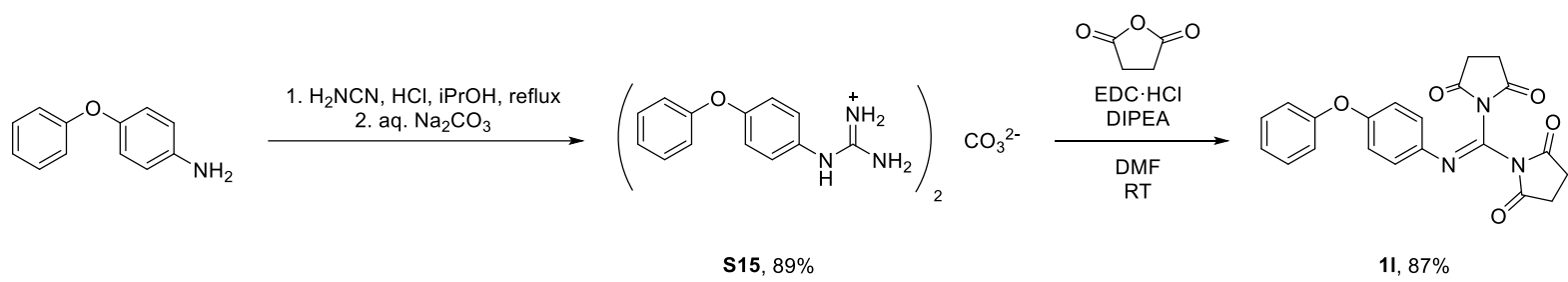

1-(4-Phenoxyphenyl)guanidine carbonate (S15)<smiles>NC([NH3+])=Nc1ccc(Oc2ccccc2)cc1</smiles>

$\mathbf{S 1 5}$

Following General Procedure P2, cyanamide $(1.68 \mathrm{~g}$, $40.0 \mathrm{mmol})$, 4-phenoxyaniline $(3.71 \mathrm{~g}, 20.0 \mathrm{mmol}), \mathrm{PrOH}$ $(20.0 \mathrm{ml})$, concentrated hydrochloric acid $(2.00 \mathrm{ml})$ and saturated $\mathrm{Na}_{2} \mathrm{CO}_{3}$ solution $(40.0 \mathrm{ml})$ yielded $\mathbf{S} 15(4.57 \mathrm{~g}$, $89 \%)$ as a white solid. ${ }^{1} \mathrm{H}$ NMR $\left(500 \mathrm{MHz}\right.$, DMSO- $\left.d_{6}\right): \delta$ 7.41-7.32 (m, 2H), 7.13-7.06 (m, 1H), 7.05-6.91 (m, 6H), 10.02-4.15 (brs, 5H). ${ }^{13} \mathrm{C}$ NMR (125 MHz, DMSO- $\left.d_{6}\right): \delta$ 157.6, 154.4, 151.6, 140.1, 129.9, 125.2, 122.8, 120.0, 117.8. MS (ESI) calculated for $\mathrm{C}_{13} \mathrm{H}_{13} \mathrm{~N}_{3} \mathrm{O}$ $[\mathrm{M}+\mathrm{H}]^{+}: \mathrm{m} / \mathrm{z} 228.1$, found 228.1 .

1,1'-(((4-Phenoxyphenyl)imino)methylene)bis(pyrrolidine-2,5-dione) (1I)<smiles>O=C1CCC(=O)N1C(=Nc1ccc(Oc2ccccc2)cc1)N1C(=O)CCC1=O</smiles>

11

$[M+H]^{+}: \mathrm{m} / \mathrm{z}$ 392.1, found 392.1.
Following General Procedure P3, S15 (2.58 g, $10.0 \mathrm{mmol})$, succinic anhydride $(3.00 \mathrm{~g}, 30.0 \mathrm{mmol}), \mathrm{EDC} \cdot \mathrm{HCl}(3.83 \mathrm{~g}, 20.0 \mathrm{mmol}), \mathrm{DMF}$ $(10.0 \mathrm{ml})$ and DIPEA $(8.59 \mathrm{ml}, 50.0 \mathrm{mmol})$ yielded $\mathbf{S} 16$ (3.41 g, 87\%) as a white solid after purification by flash column chromatography $\left(\mathrm{SiO}_{2}\right.$, hexane/ethyl acetate $5: 1 \rightarrow$ hexane/ethyl acetate $\left.1: 3\right) .{ }^{1} \mathrm{H}$ NMR $\left(500 \mathrm{MHz}, \mathrm{CDCl}_{3}\right): \delta$ 7.37-7.27 (m, 2H), 7.12-7.05 (m, 1H), 7.00-6.88 (m, 6H), $2.81(\mathrm{~s}, 4 \mathrm{H}), 2.66(\mathrm{~s}, 4 \mathrm{H}) .{ }^{13} \mathrm{C} \mathrm{NMR}(125 \mathrm{MHz}$, $\left.\mathrm{CDCl}_{3}\right): \delta 173.7,173.0,157.0,155.3,139.9,129.8,129.3,123.5$, 121.6, 119.3, 118.9, 28.3, 28.2. MS (ESI)+ calculated for $\mathrm{C}_{21} \mathrm{H}_{18} \mathrm{~N}_{3} \mathrm{O}_{5}$ 


\section{Synthesis of 8}

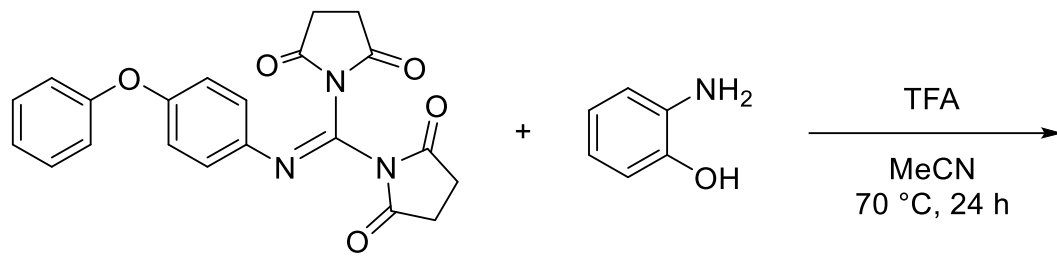

11<smiles>c1ccc(Oc2ccc(Nc3nc4ccccc4o3)cc2)cc1</smiles>

$8,29 \%$

$\alpha$-Glucosidase inhibitor $\left(\mathrm{IC}_{50}=32.49 \mathrm{uM}\right)$

To a stirred solution of 1 ( (783 mg, $2.00 \mathrm{mmol}, 1.00$ eq.) and TFA ( $0.157 \mathrm{ml}, 2.00 \mathrm{mmol}, 1.00$ eq.) in MeCN (8.00 ml), 2-aminophenol ( $661.5 \mathrm{mg}, 1.50 \mathrm{mmol}, 3.00$ eq.) was added, then the reaction mixture was placed in an oil bath and stirred at $70^{\circ} \mathrm{C}$. After $24 \mathrm{~h}$ reaction, the reaction mixture was concentrated under reduced pressure. $\alpha$-Glucosidase inhibitor 8 was afforded (173 mg, 29\%) as a brown solid after purification by flash column chromatography $\left(\mathrm{SiO}_{2}\right.$, hexane/ethyl acetate 10:1 $\rightarrow$ hexane/ethyl acetate 4:1). ${ }^{1} \mathrm{H}$ NMR (500 MHz, $\left.\mathrm{CDCl}_{3}\right): \delta 9.14$ (brs, $\left.1 \mathrm{H}\right), 7.60(\mathrm{~d}, J=8.8 \mathrm{~Hz}, 2 \mathrm{H}), 7.47(\mathrm{~d}, J=7.7 \mathrm{~Hz}, 1 \mathrm{H})$, 7.41-7.33 (m, 3H), 7.29-7.23 (m, 1H), 7.17-7.08 (m, 4H), $7.05(\mathrm{~d}, J=7.9 \mathrm{~Hz}, 2 \mathrm{H}) .{ }^{13} \mathrm{C}$ NMR $(125 \mathrm{MHz}$, $\left.\mathrm{CDCl}_{3}\right): \delta 159.2,157.8,153.0,148.0,142.0,133.7,129.9,124.5,123.2,121.8,120.7,120.2,118.5$, 116.7, 109.3. MS (ESI) calculated for $\mathrm{C}_{19} \mathrm{H}_{15} \mathrm{~N}_{2} \mathrm{O}_{2}[\mathrm{M}+\mathrm{H}]^{+}: \mathrm{m} / \mathrm{z} 303.1$, found 303.0. Spectral data match those previously reported ${ }^{12}$. 

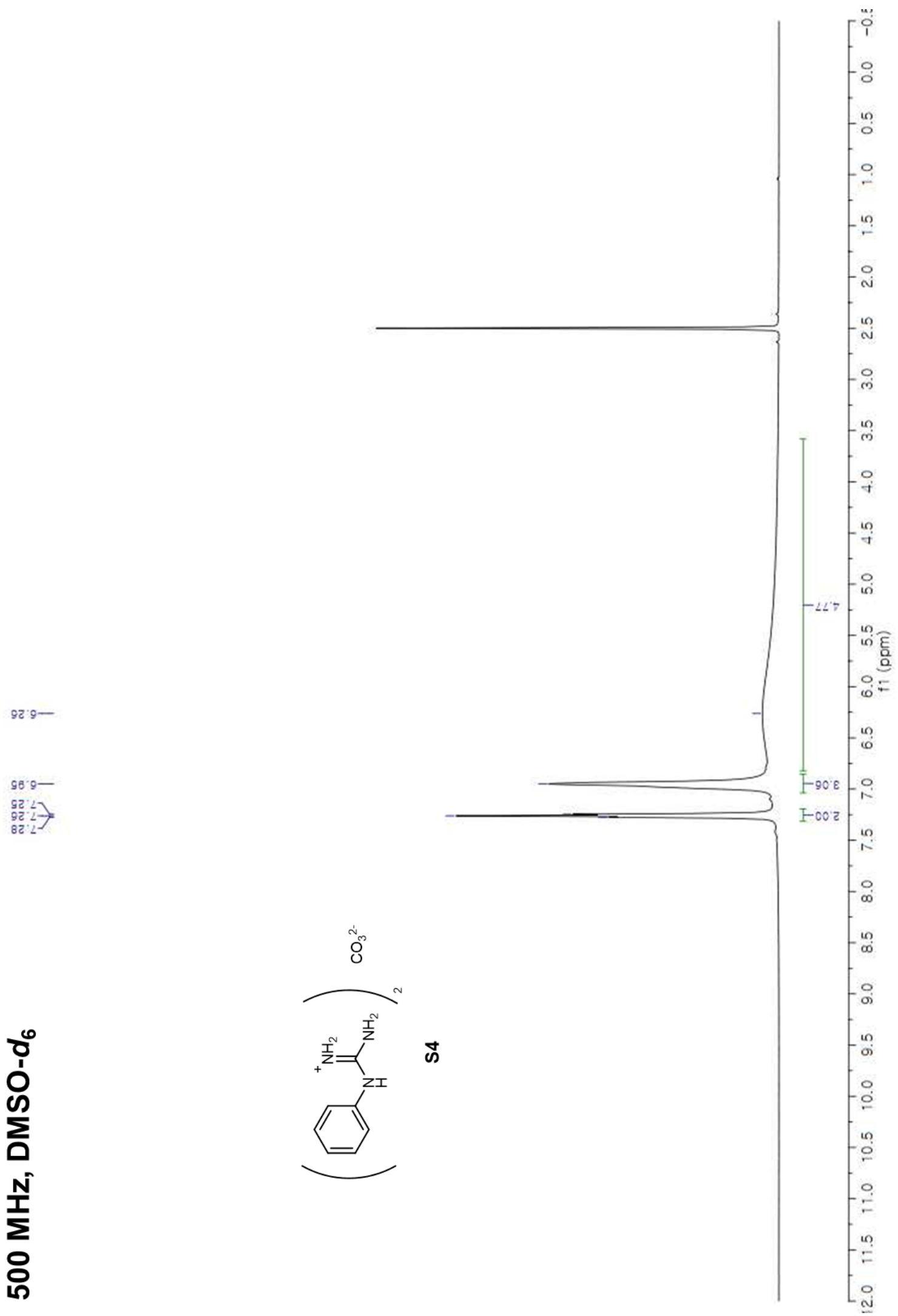


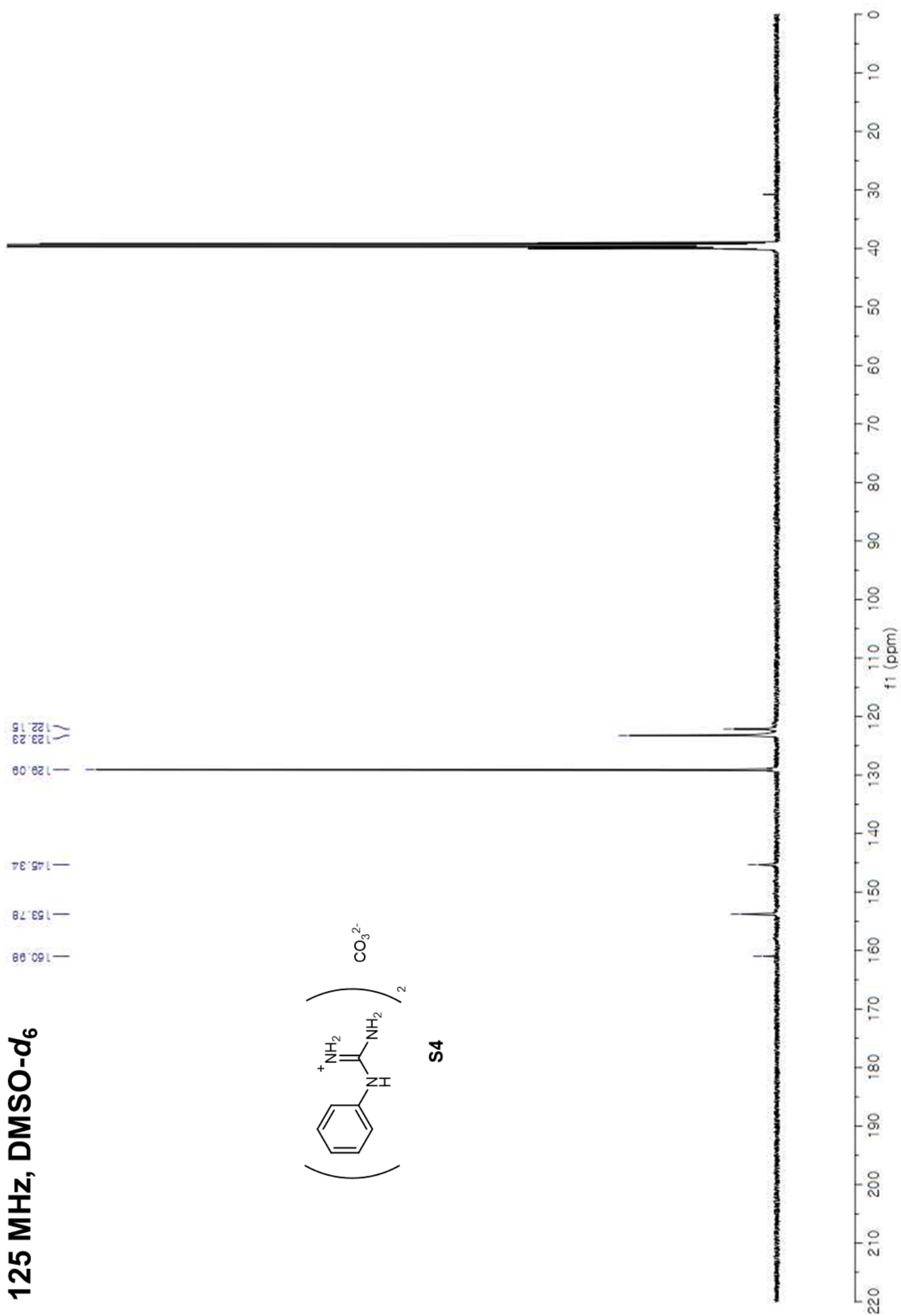




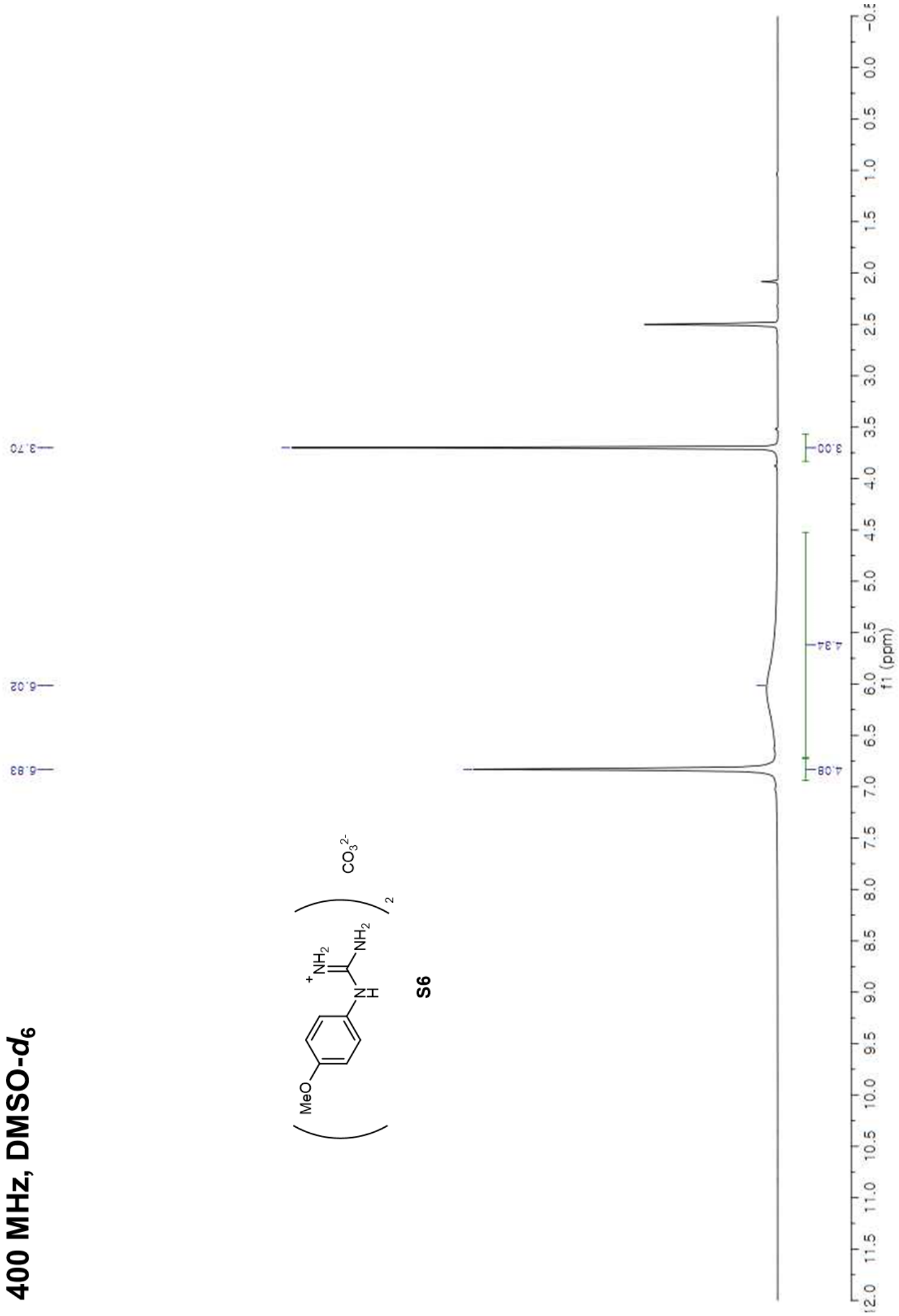




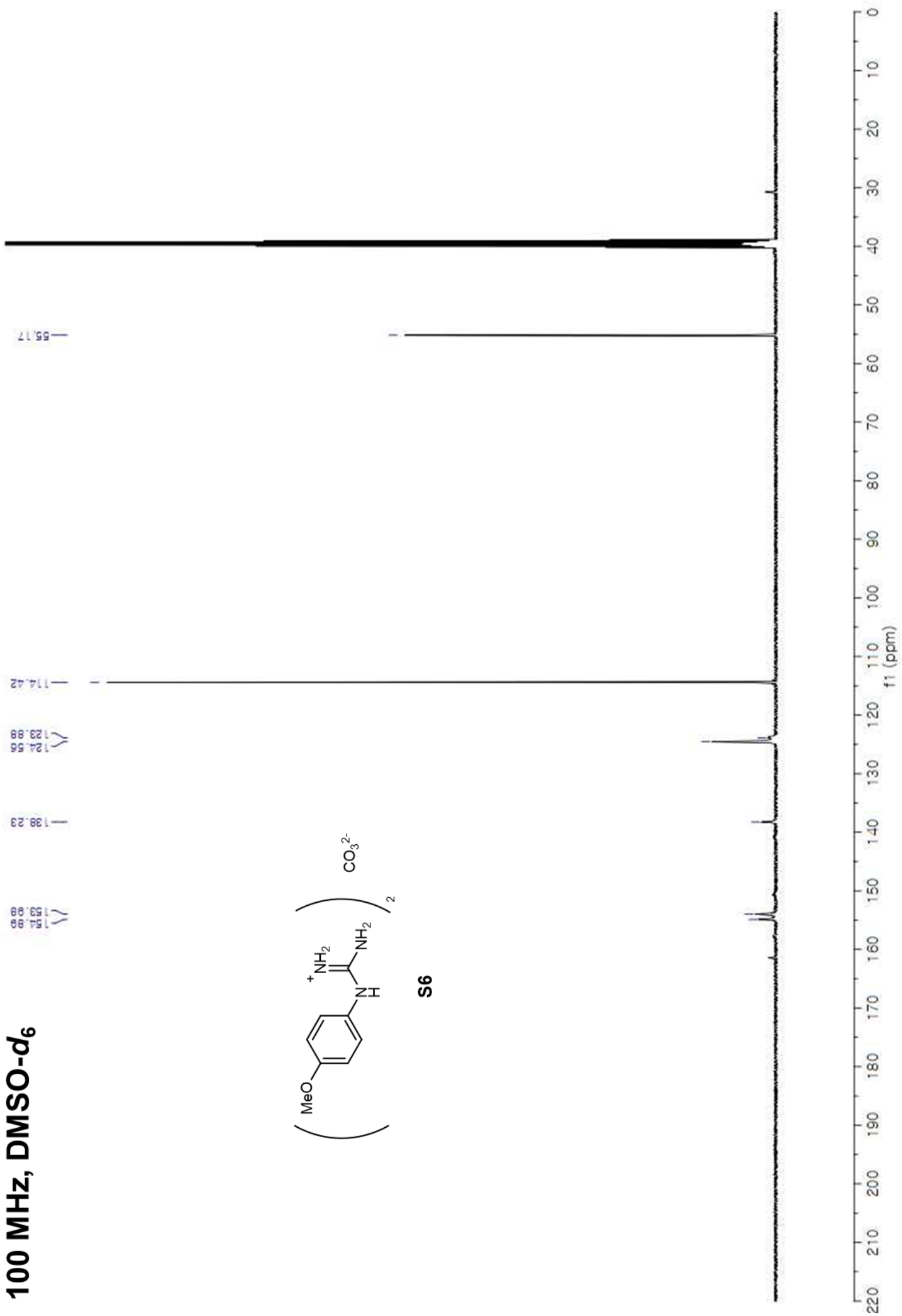




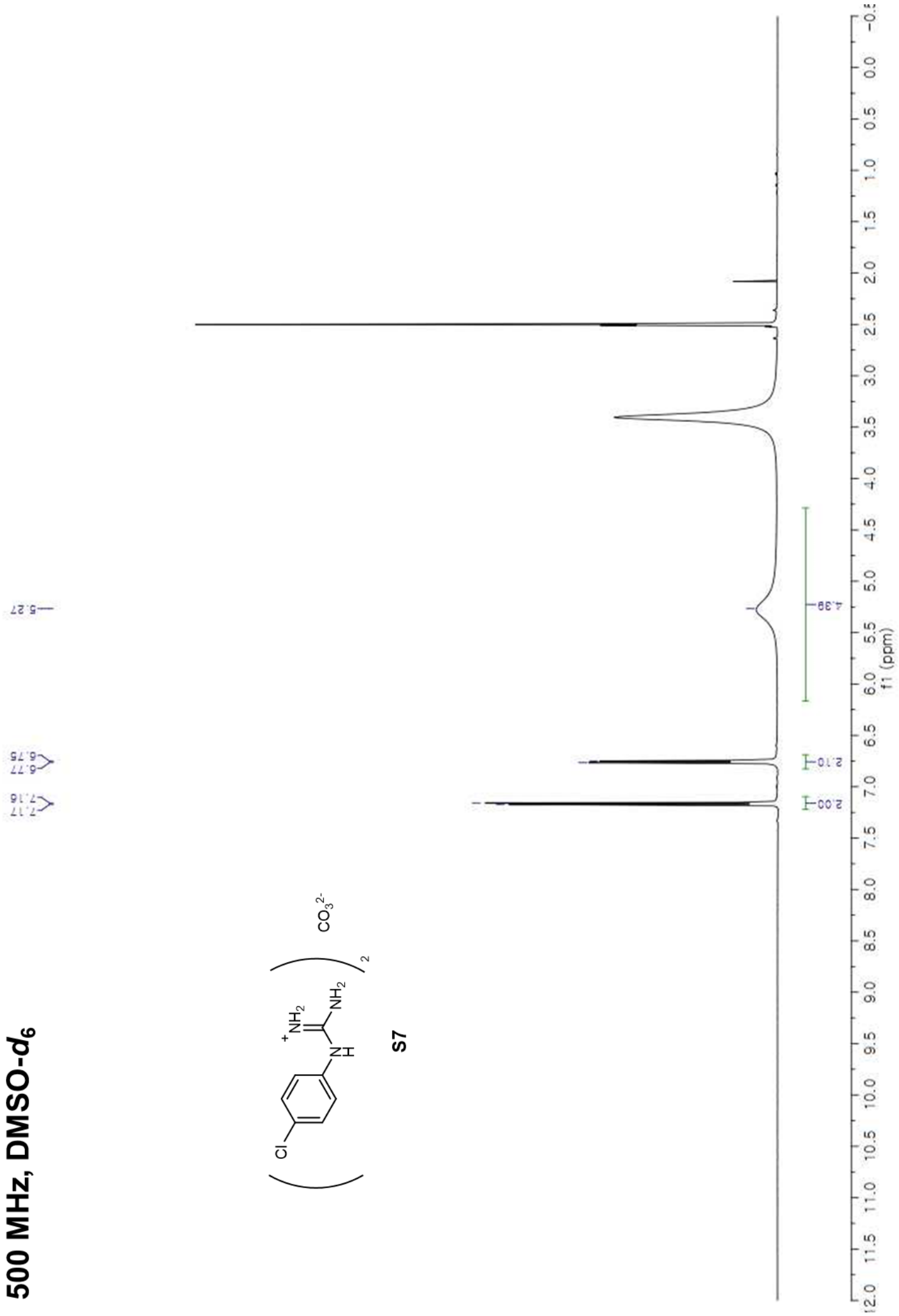




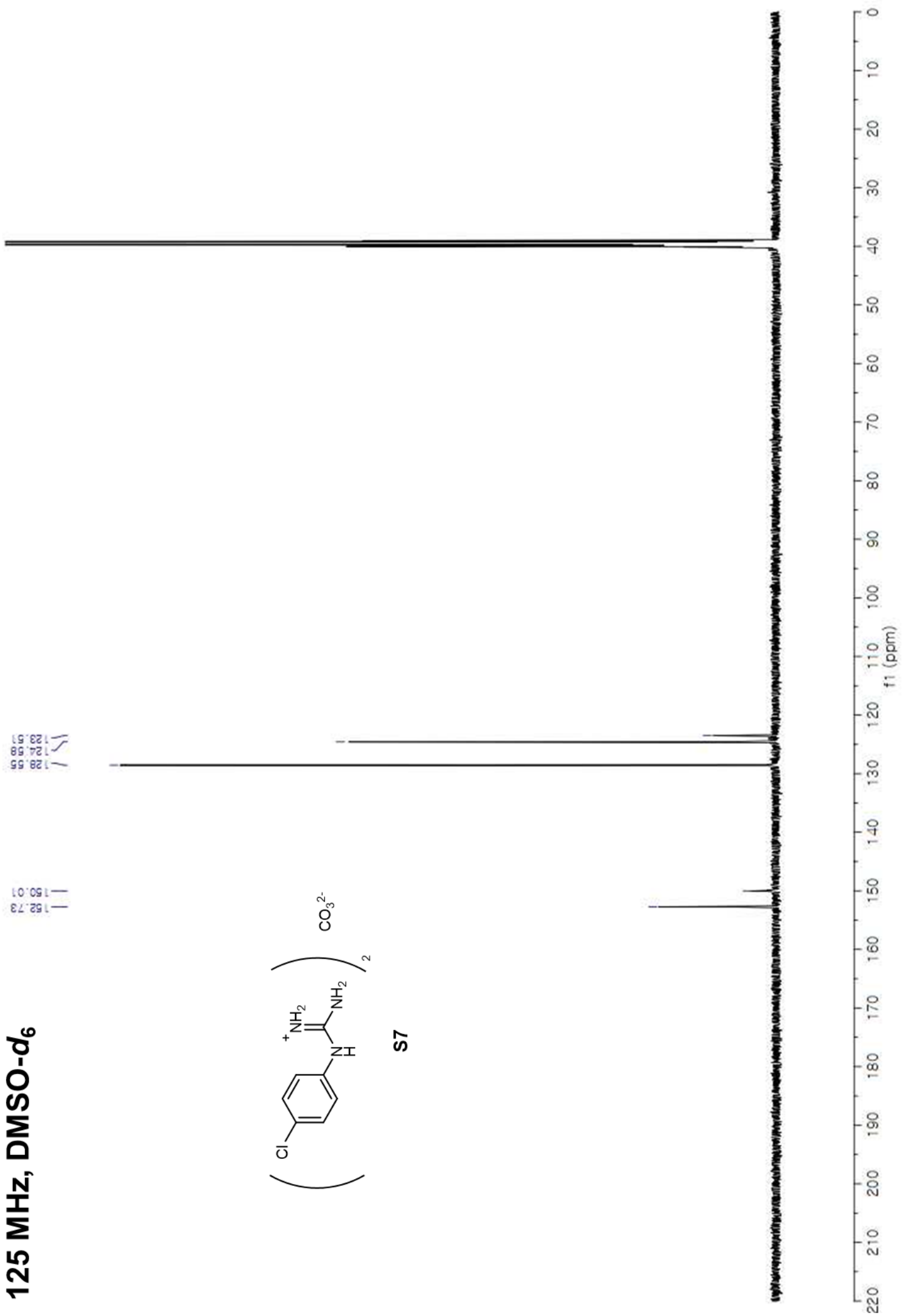




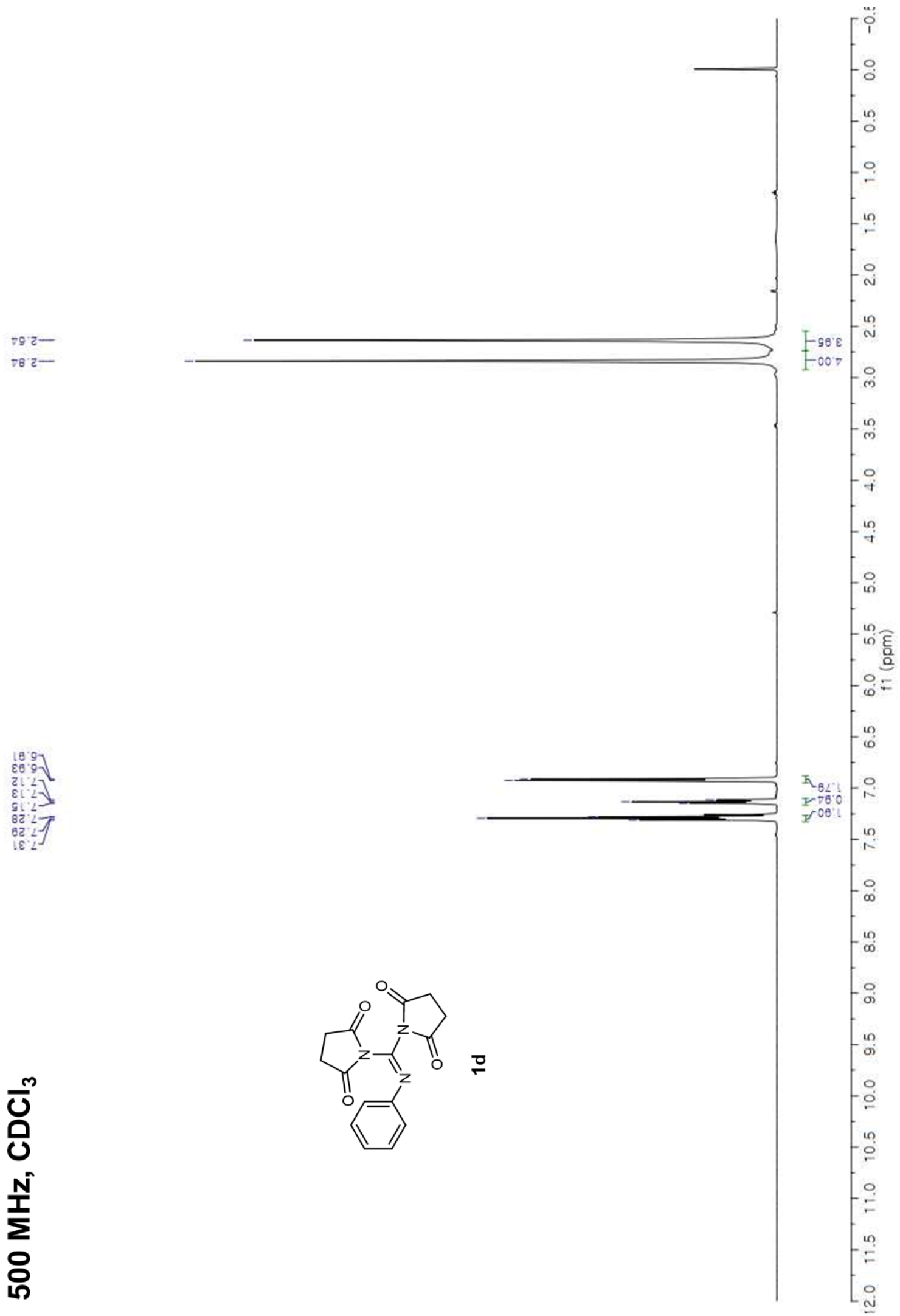




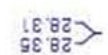
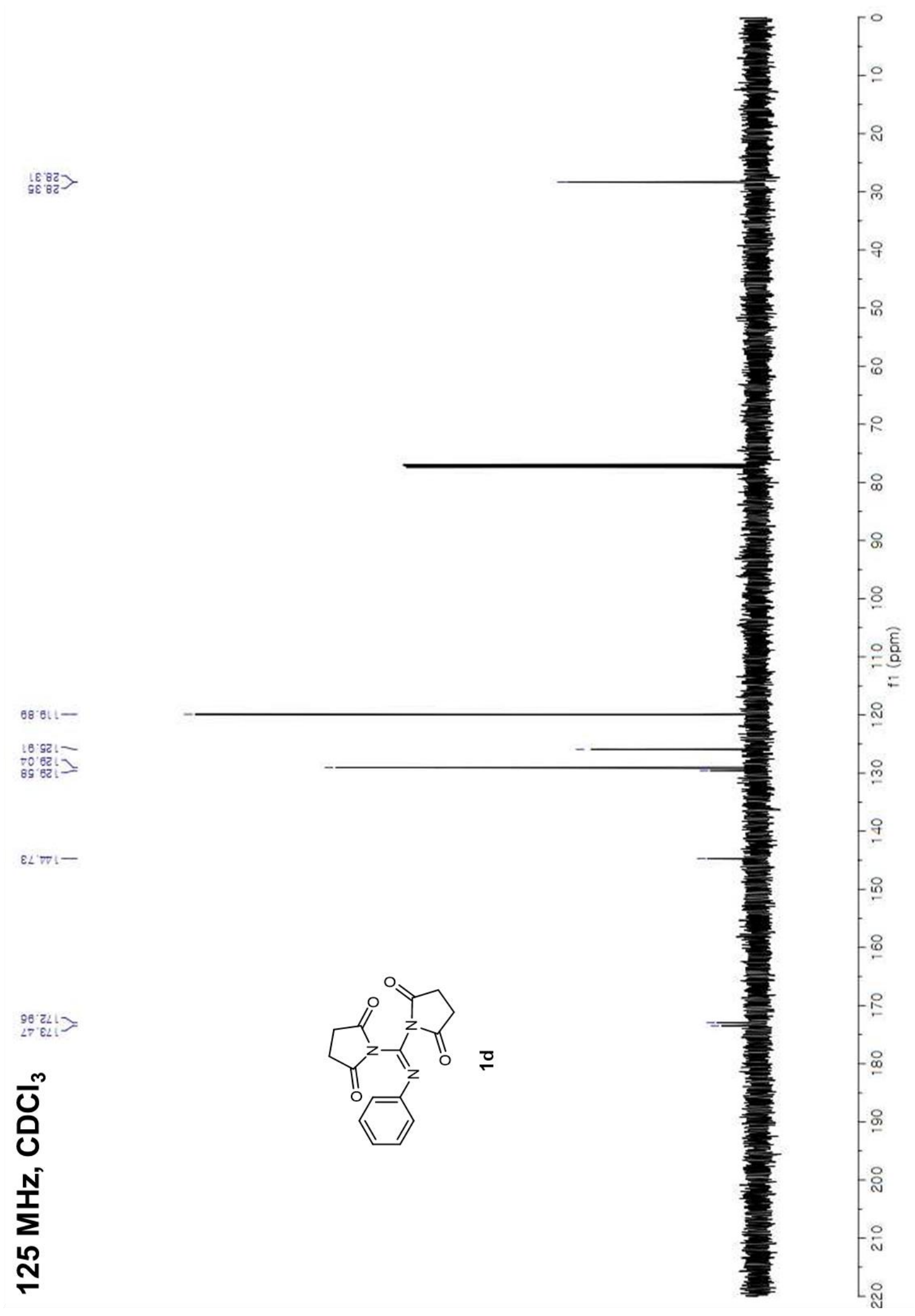


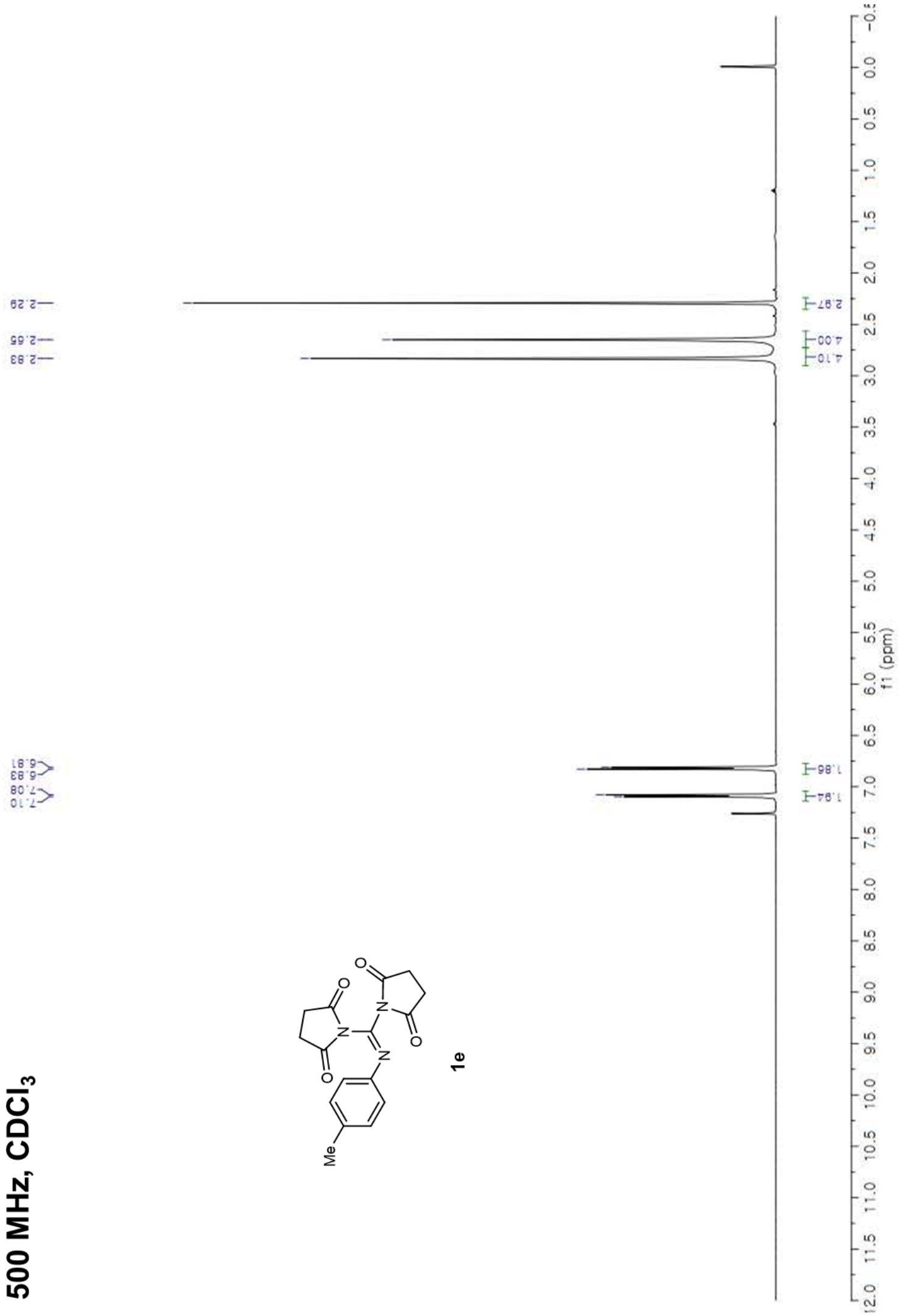



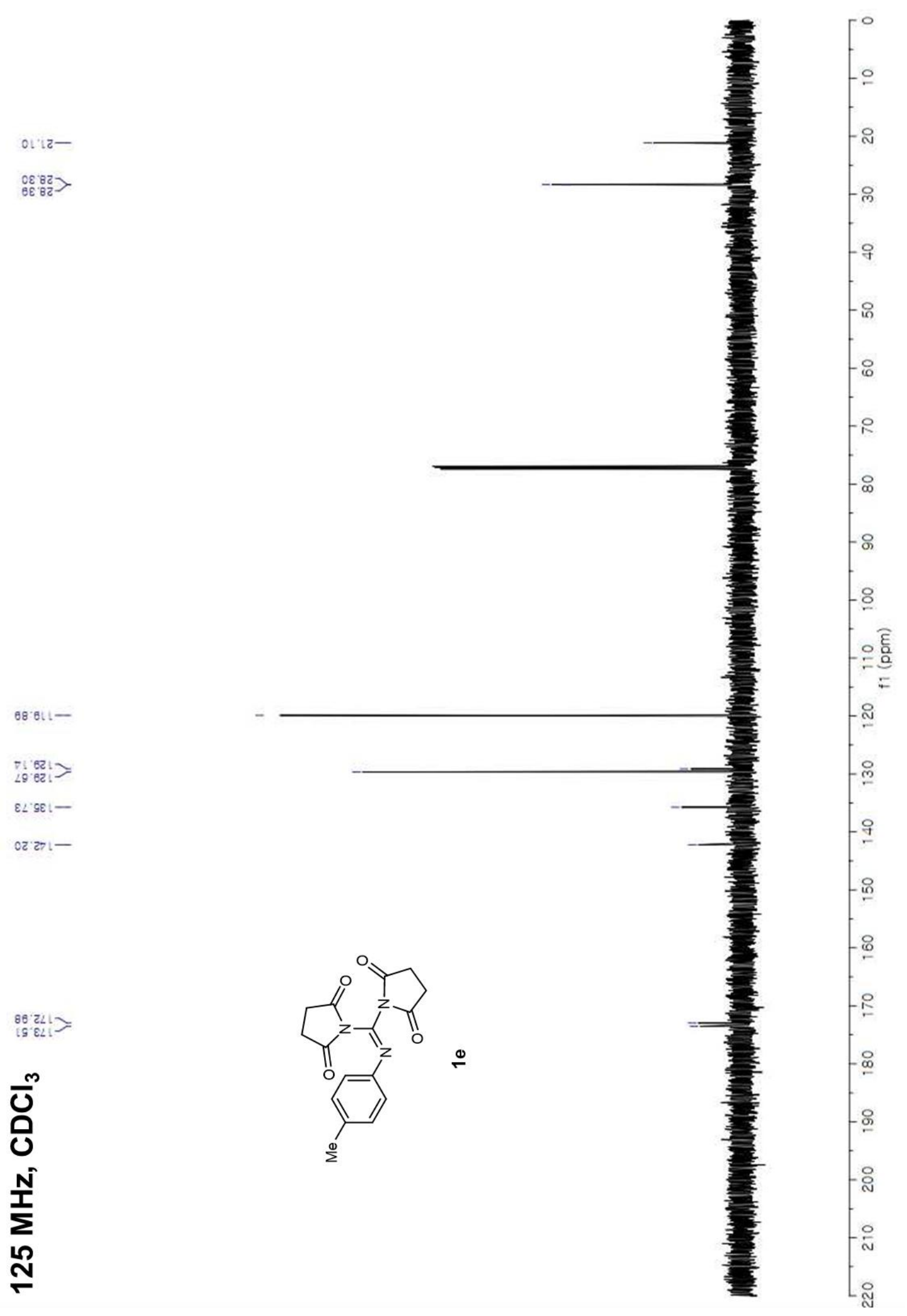


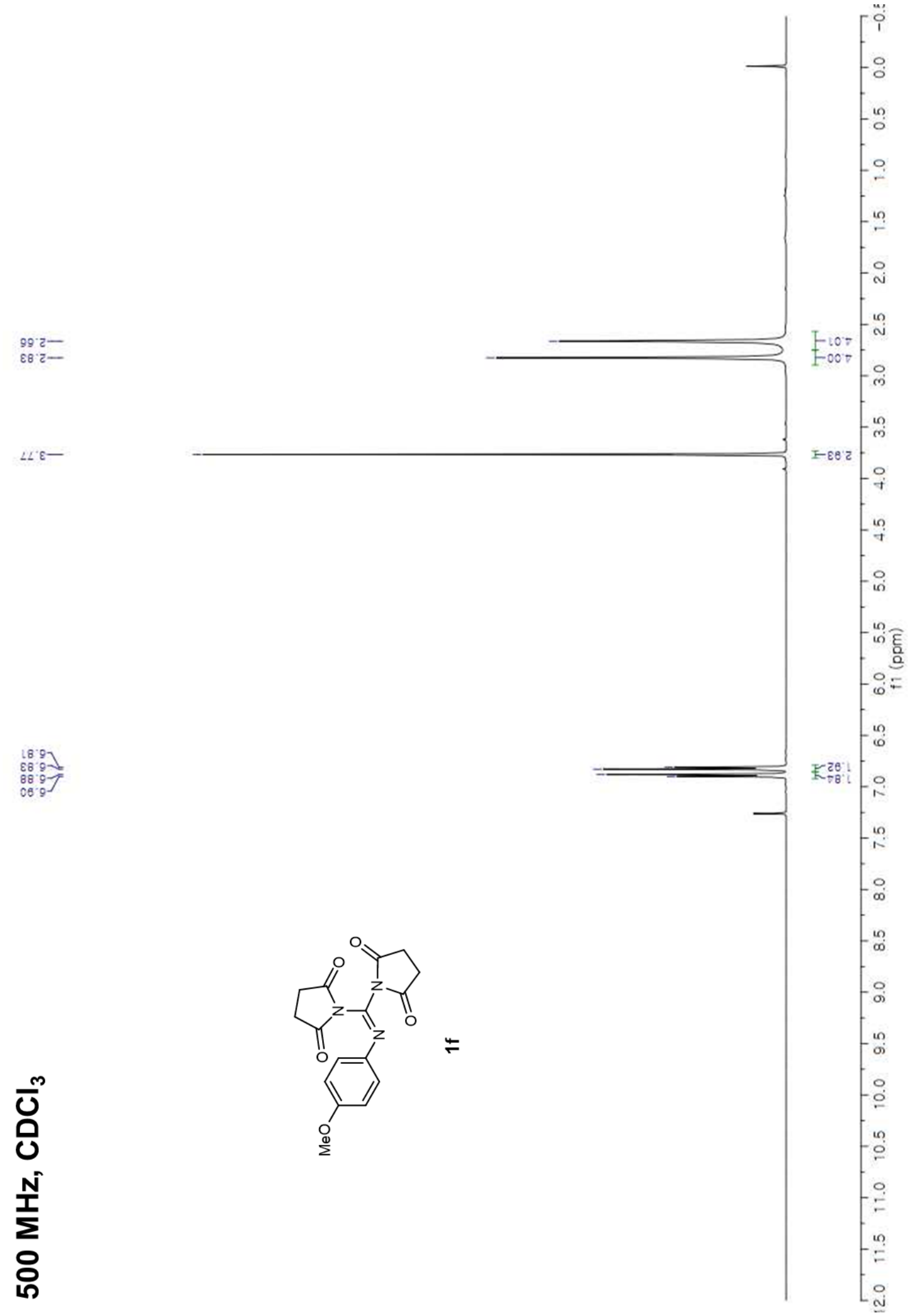




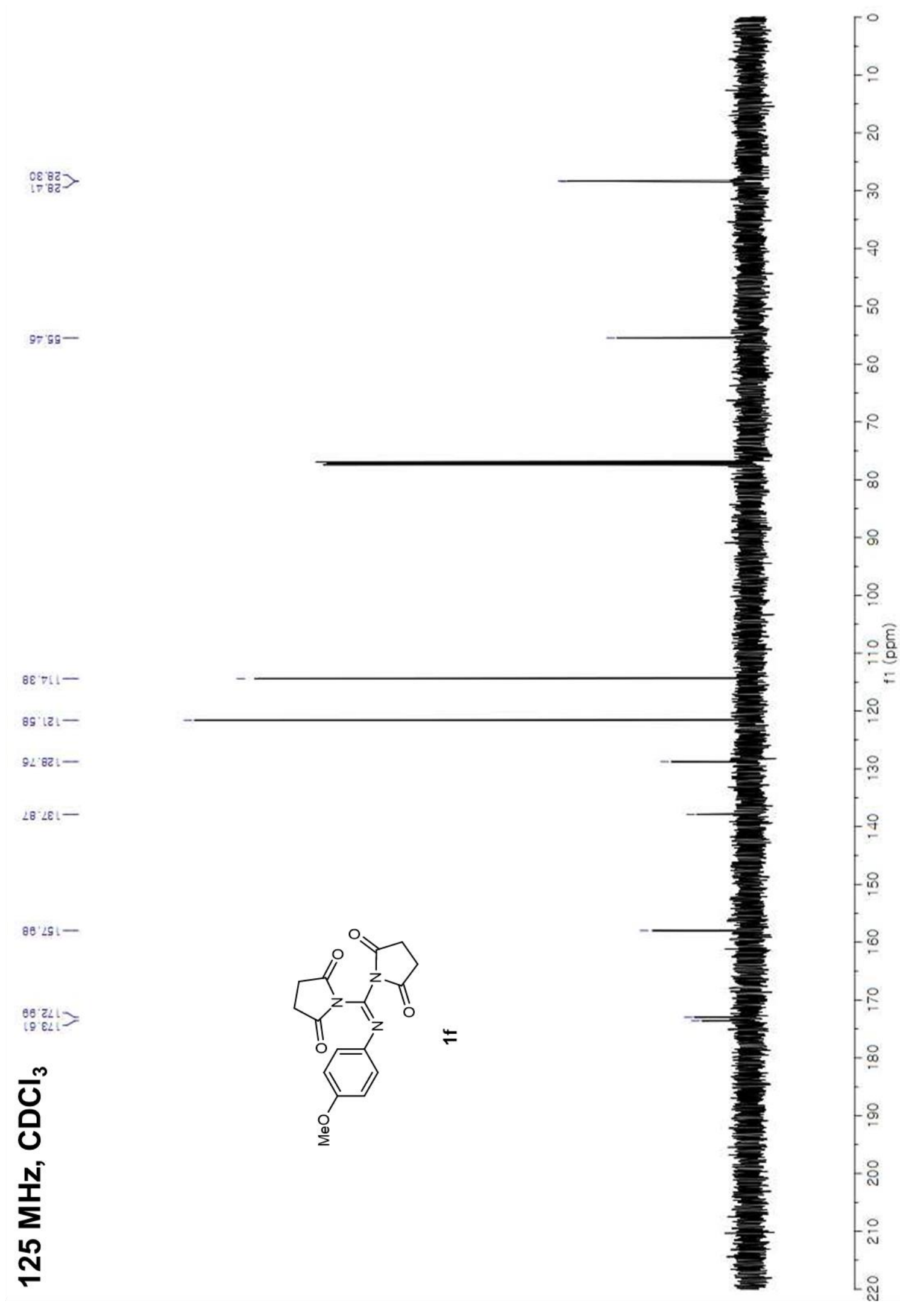


99 2-

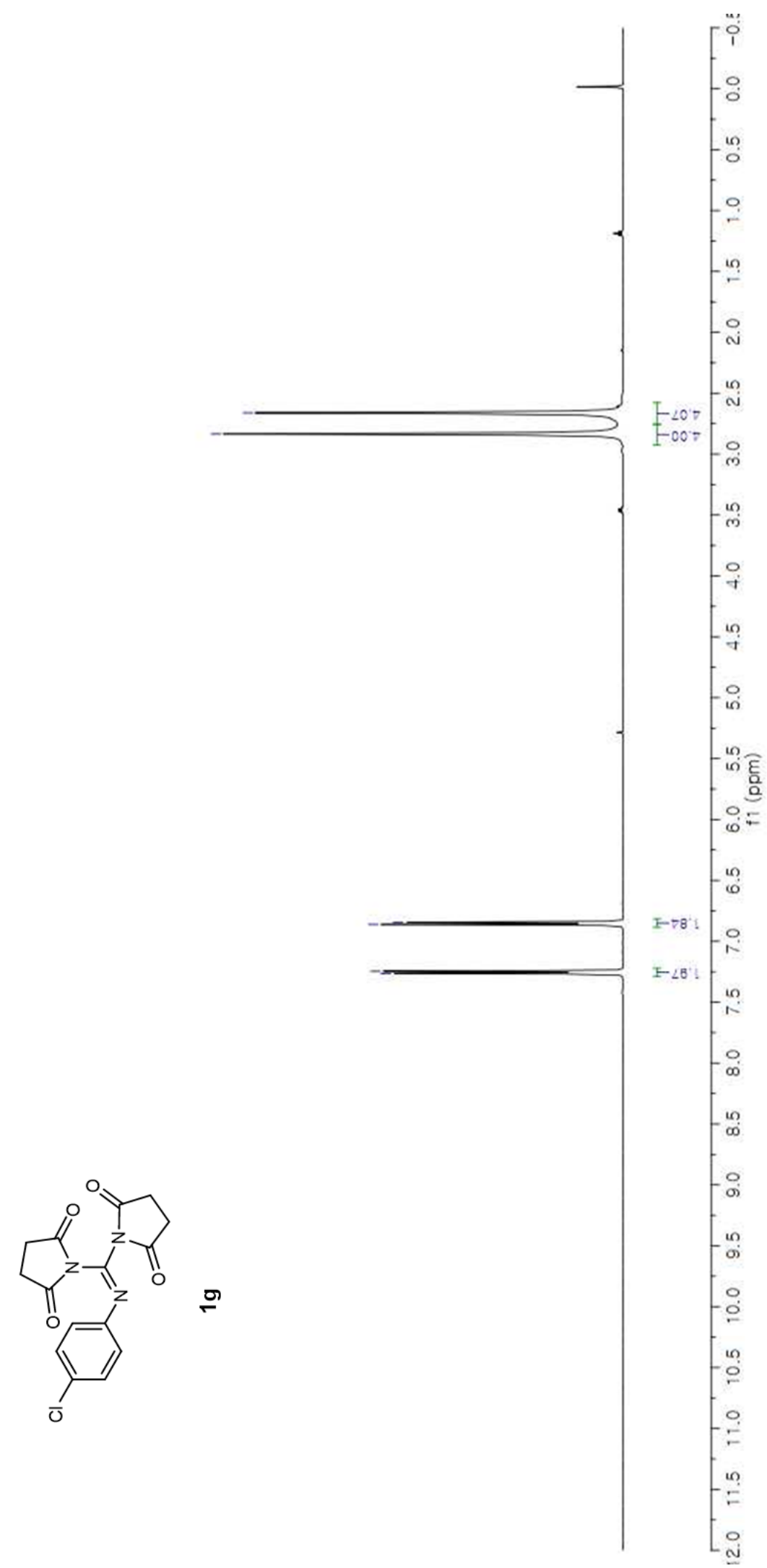

$\frac{m}{0}$
0
ํ.
ํ.
8
0

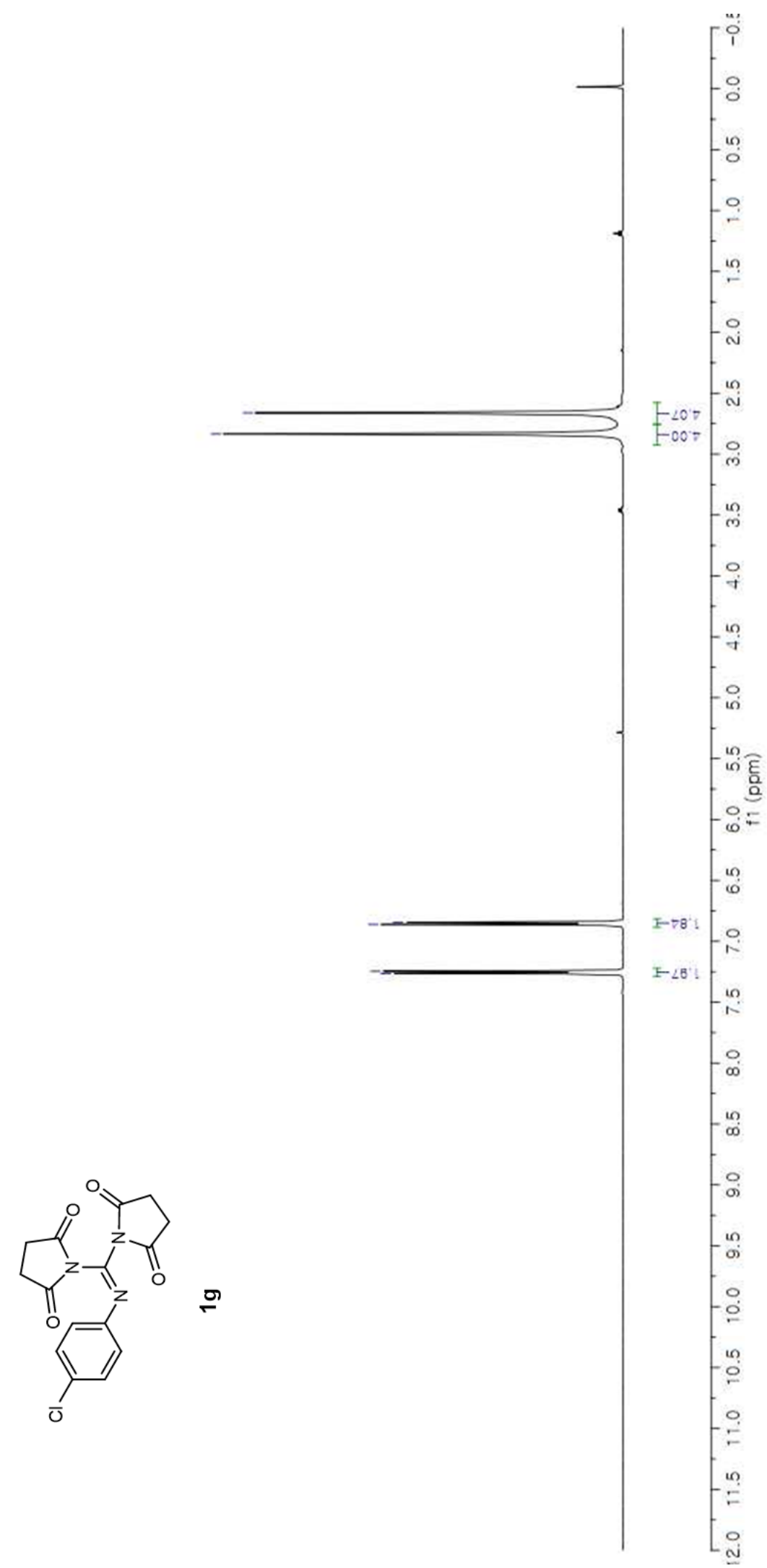

${ }_{98}^{78.9} 7$

${ }_{92}^{92} 2>$ 


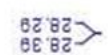

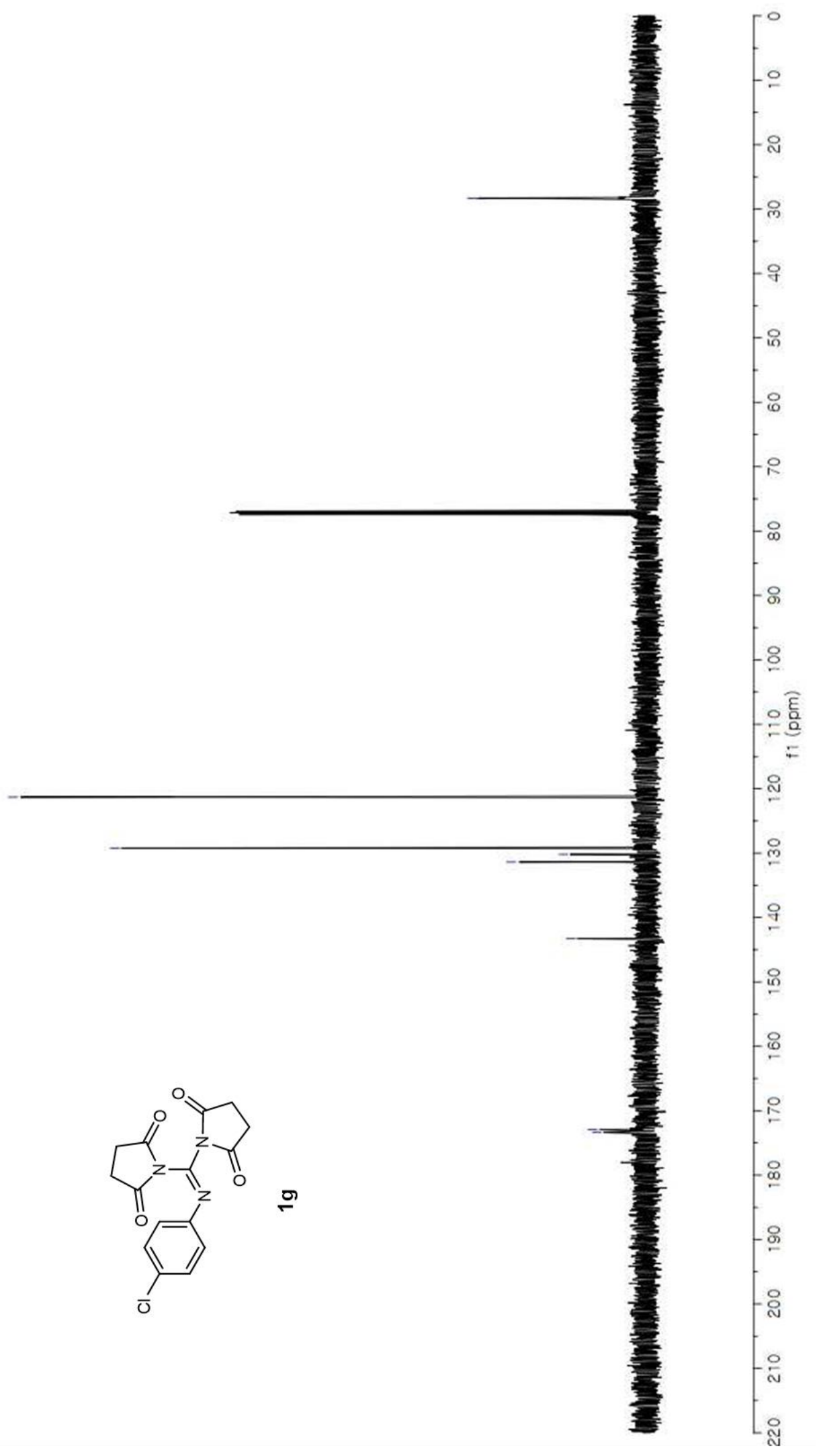

${ }_{28}^{28: 2 L}=$

$\bar{N}$
U
$\stackrel{N}{1}$
$\stackrel{N}{\Sigma}$ 


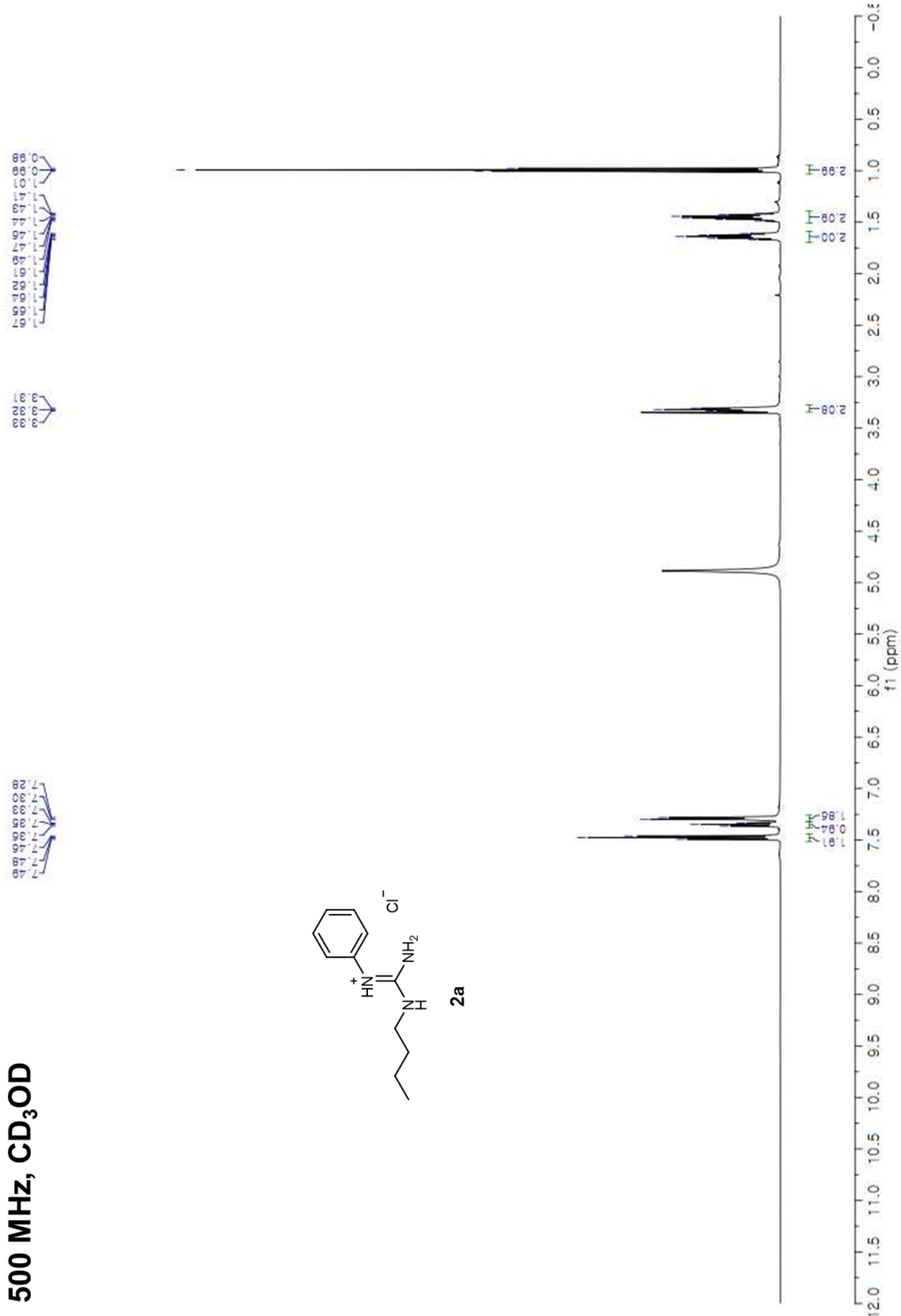



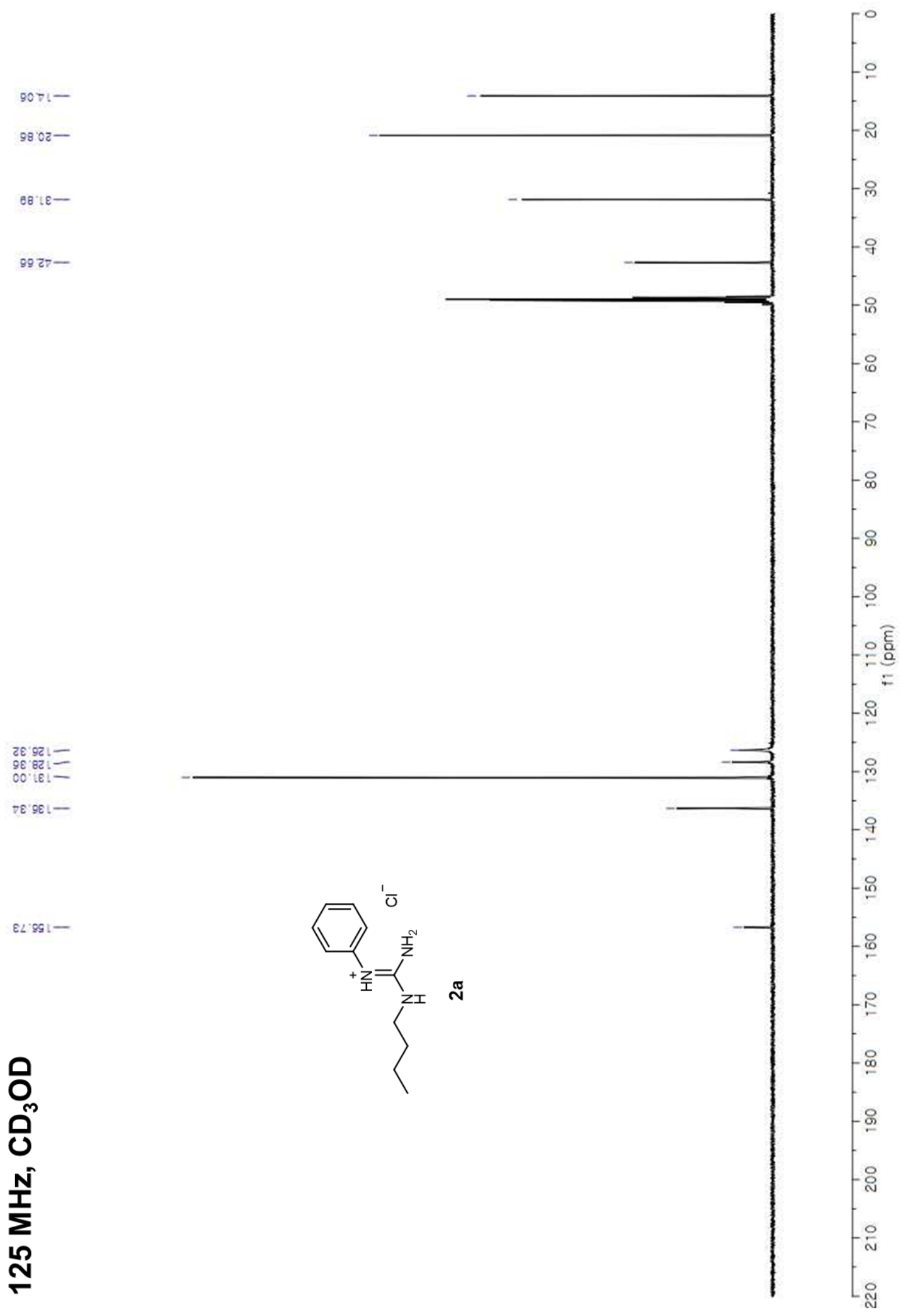


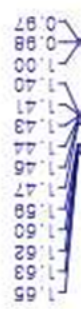

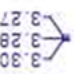

$28 \mathrm{E}-$

2027

$\begin{array}{ll}80 \\ 02 \\ 28 & 2\end{array}$

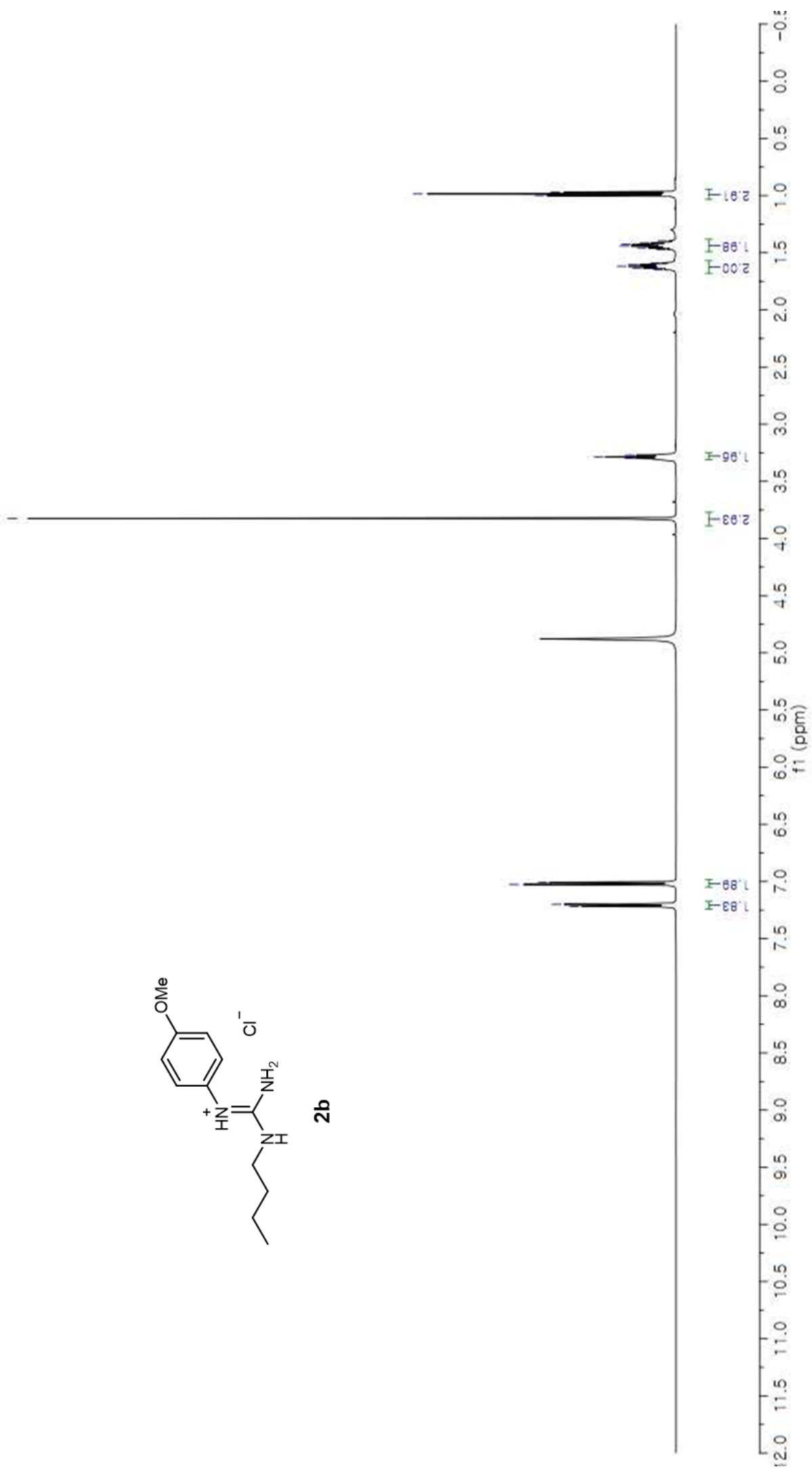




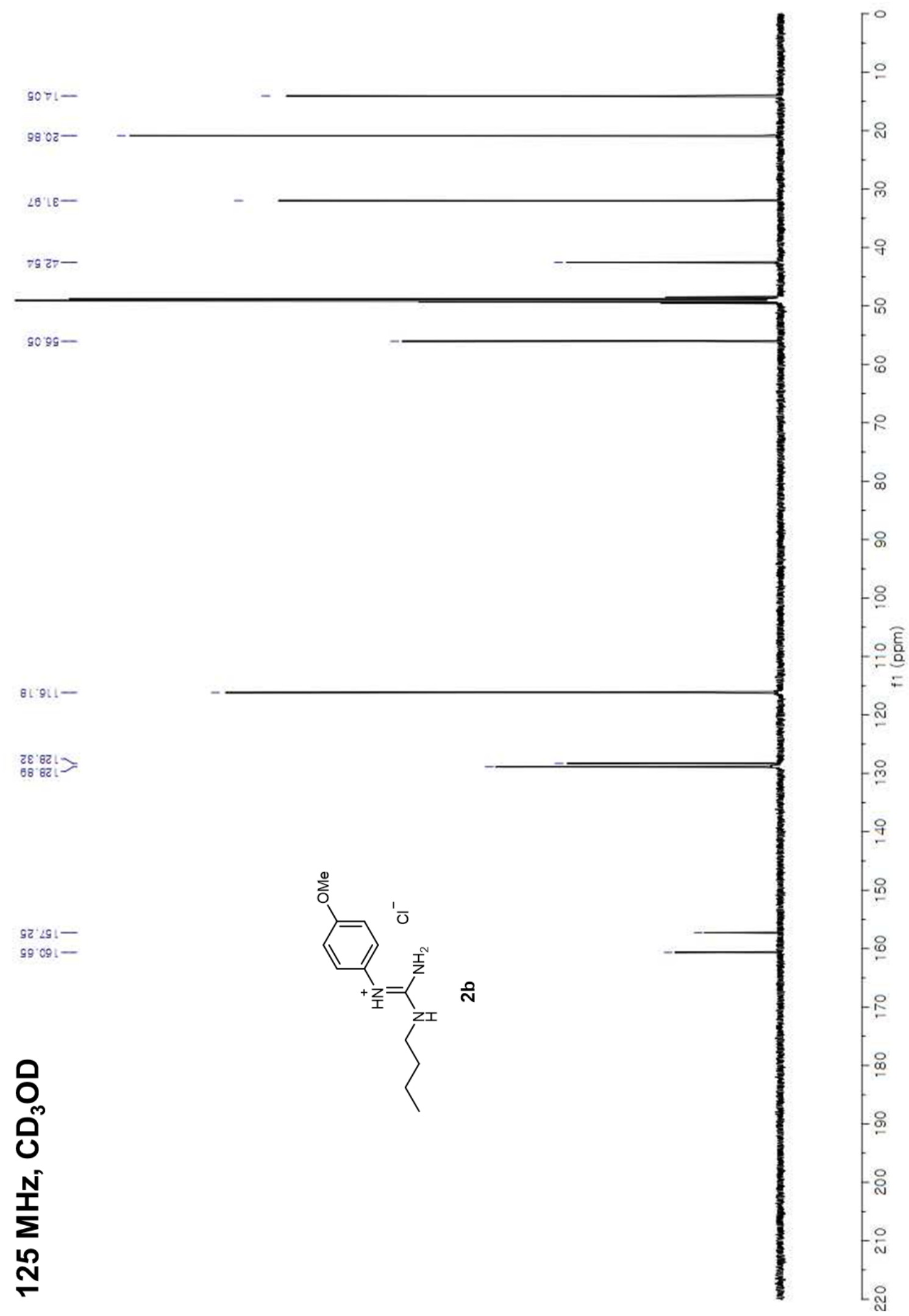




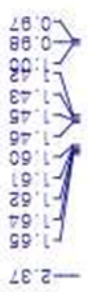

ser

$9122]$
$21: 2]$
$82: 2]$

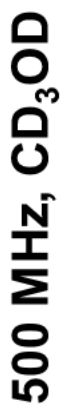

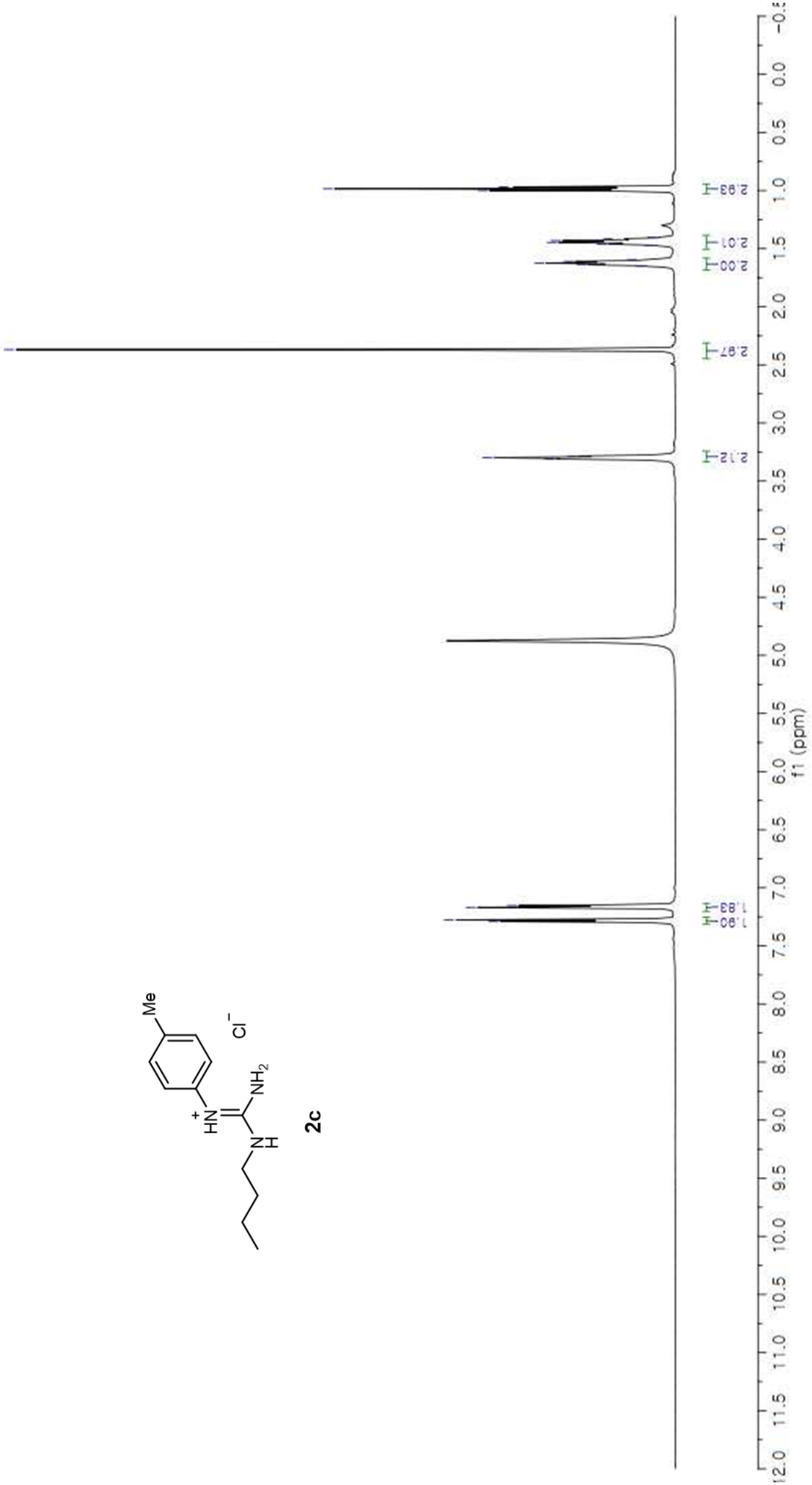



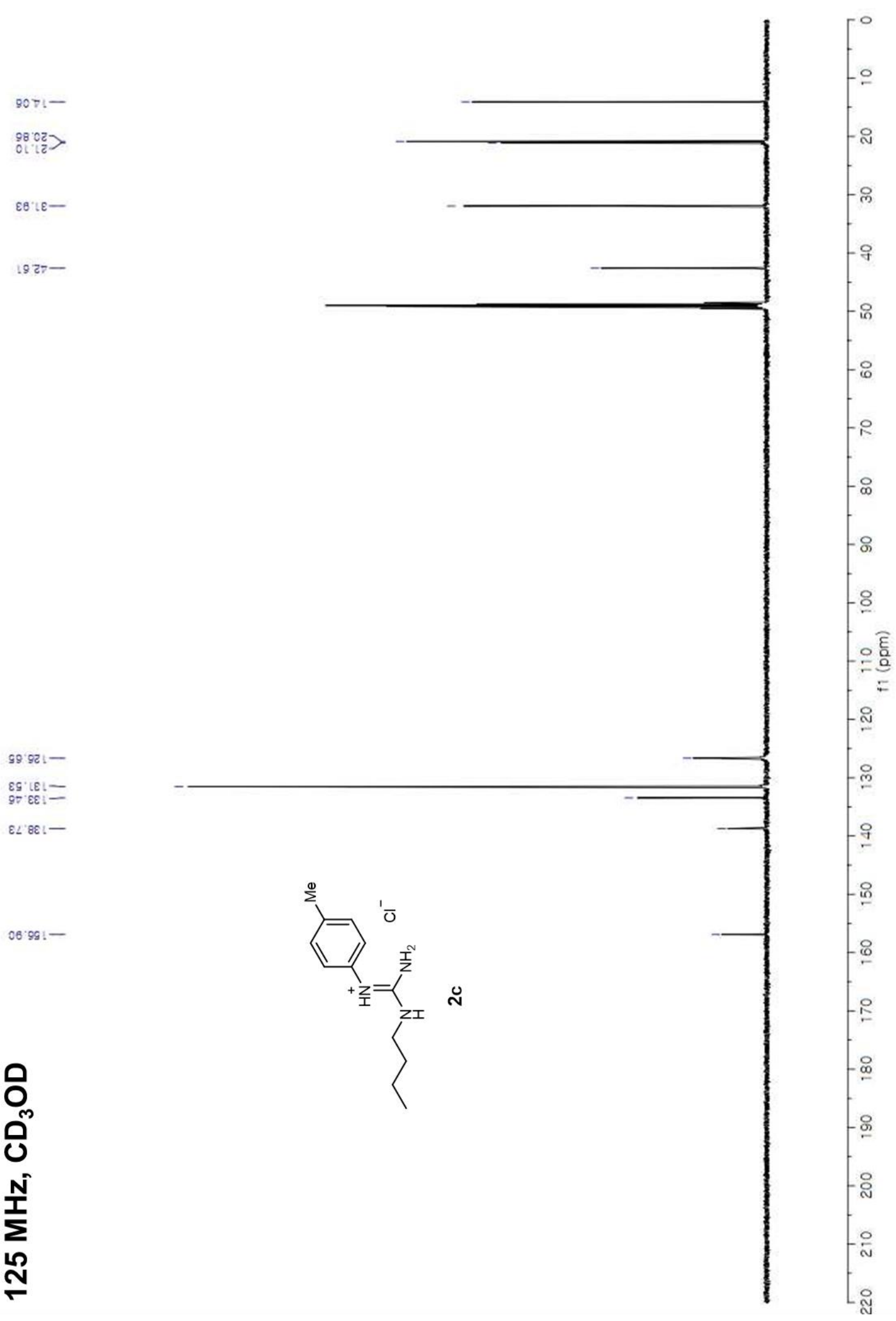

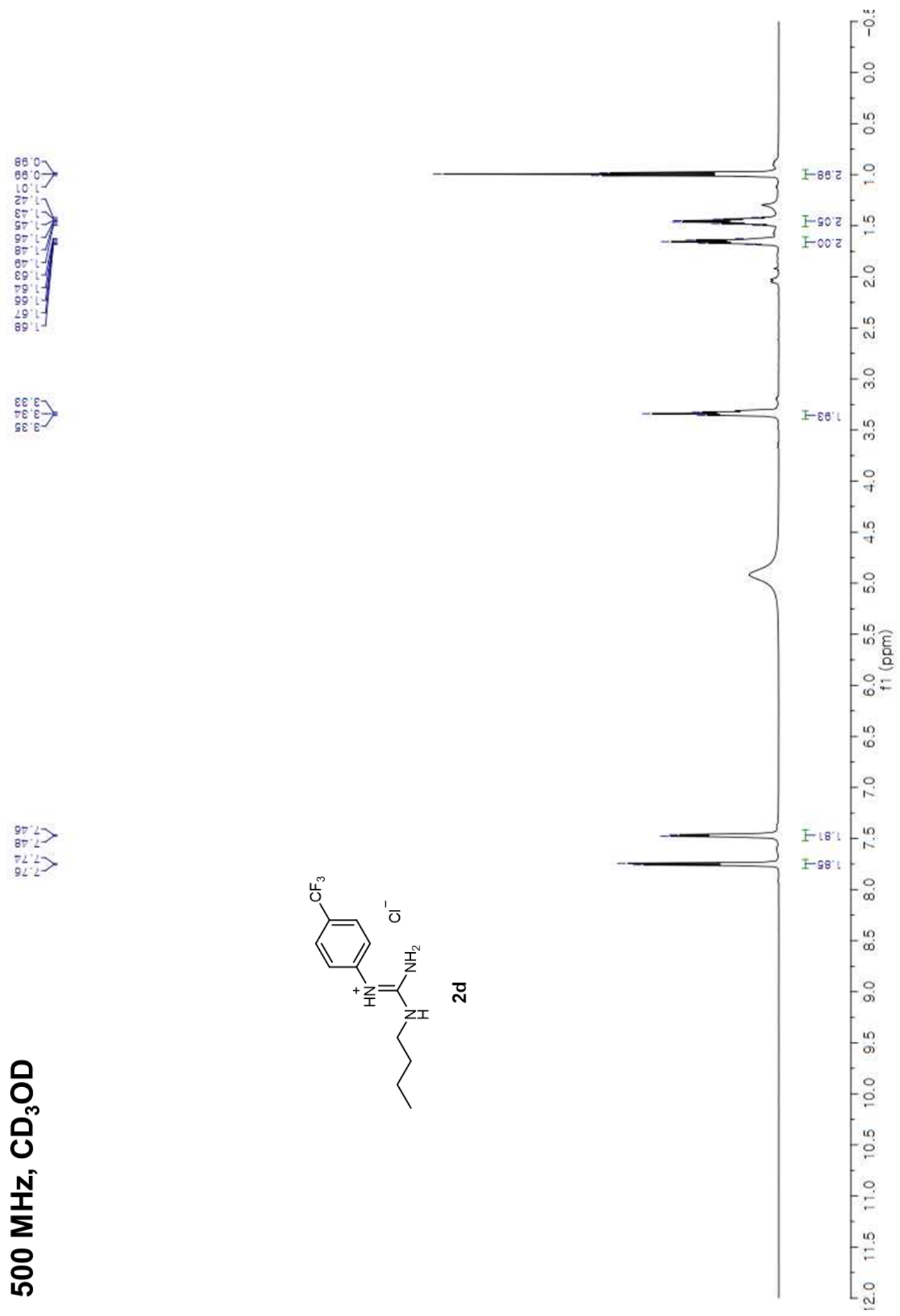


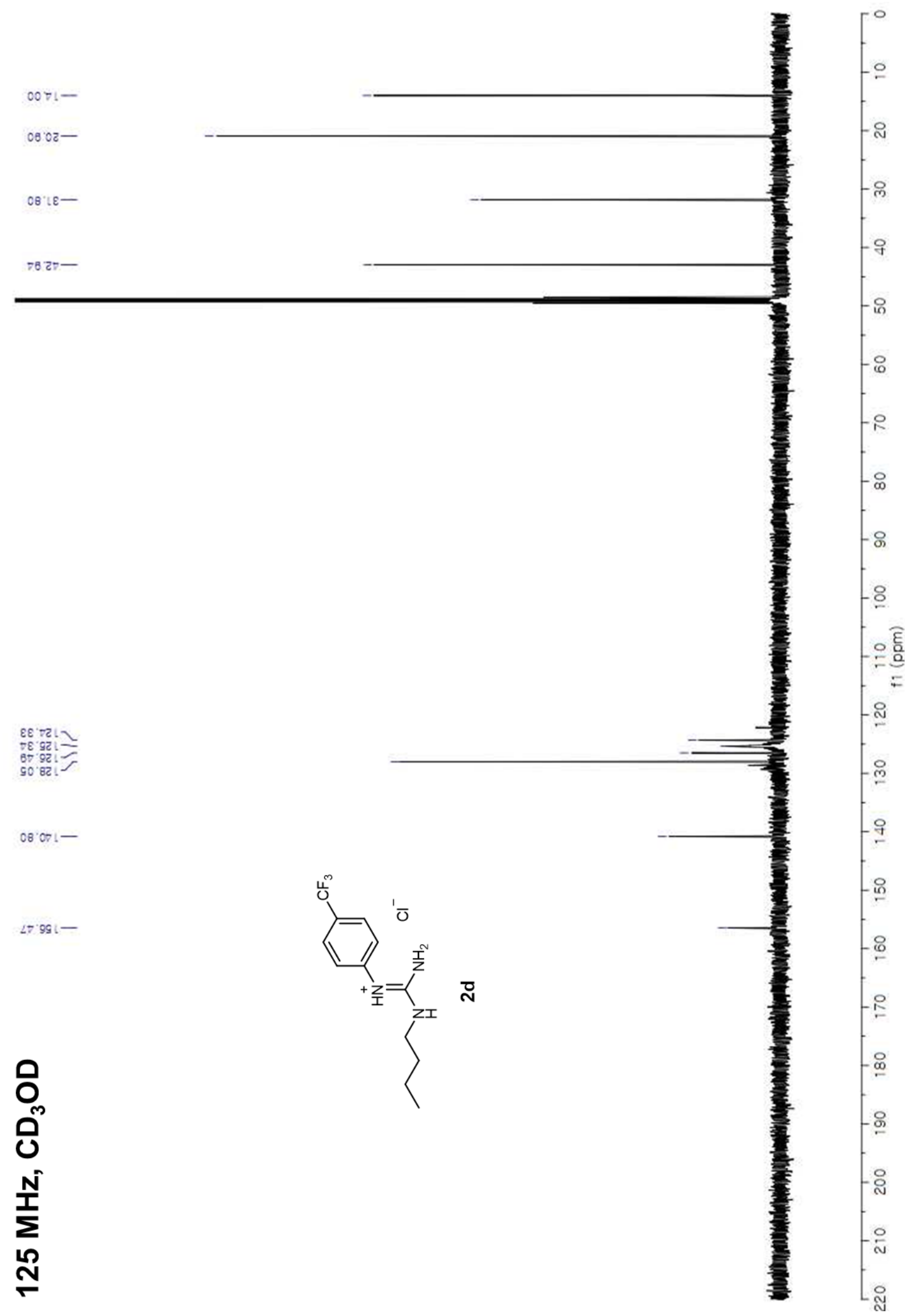



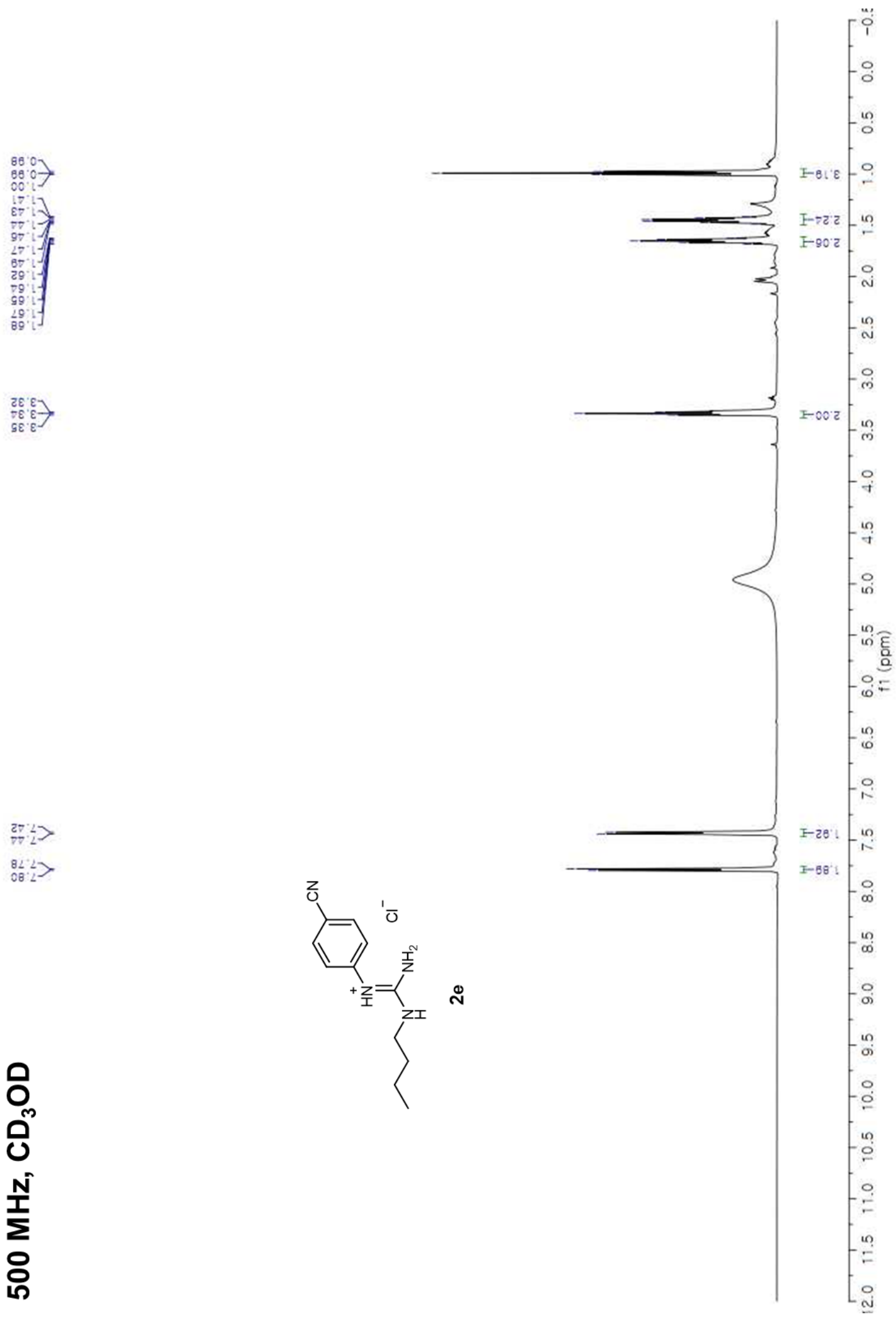

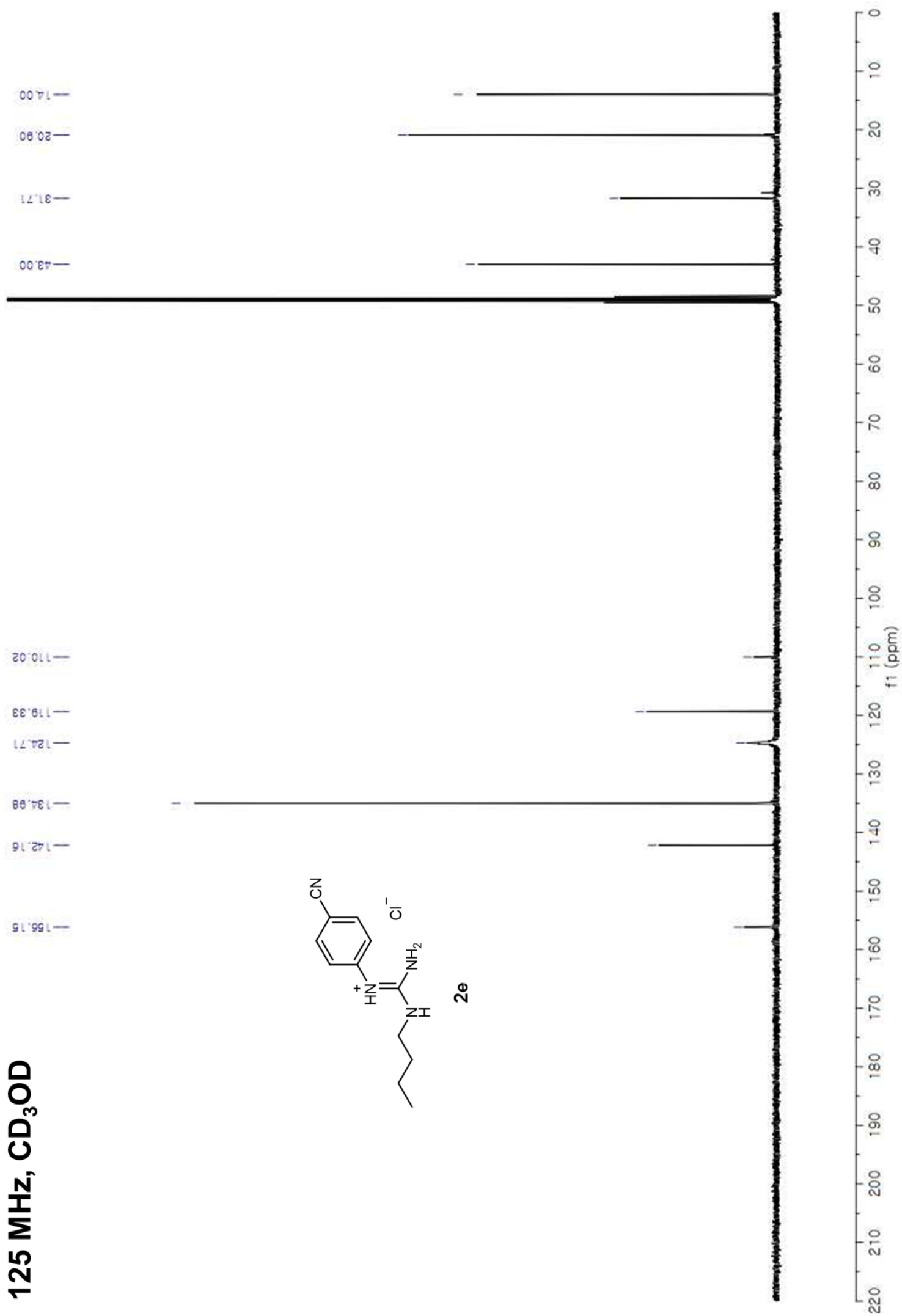

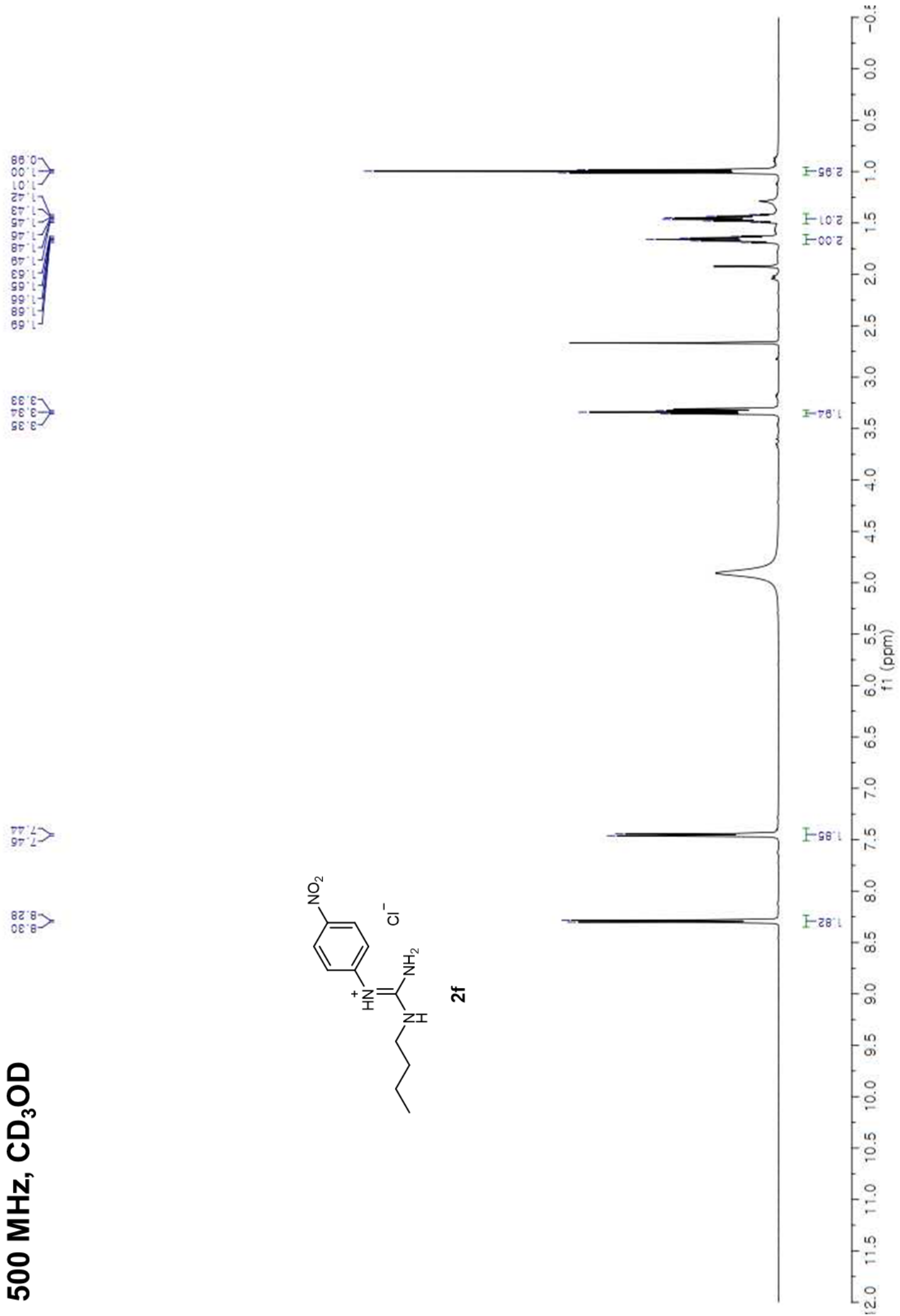


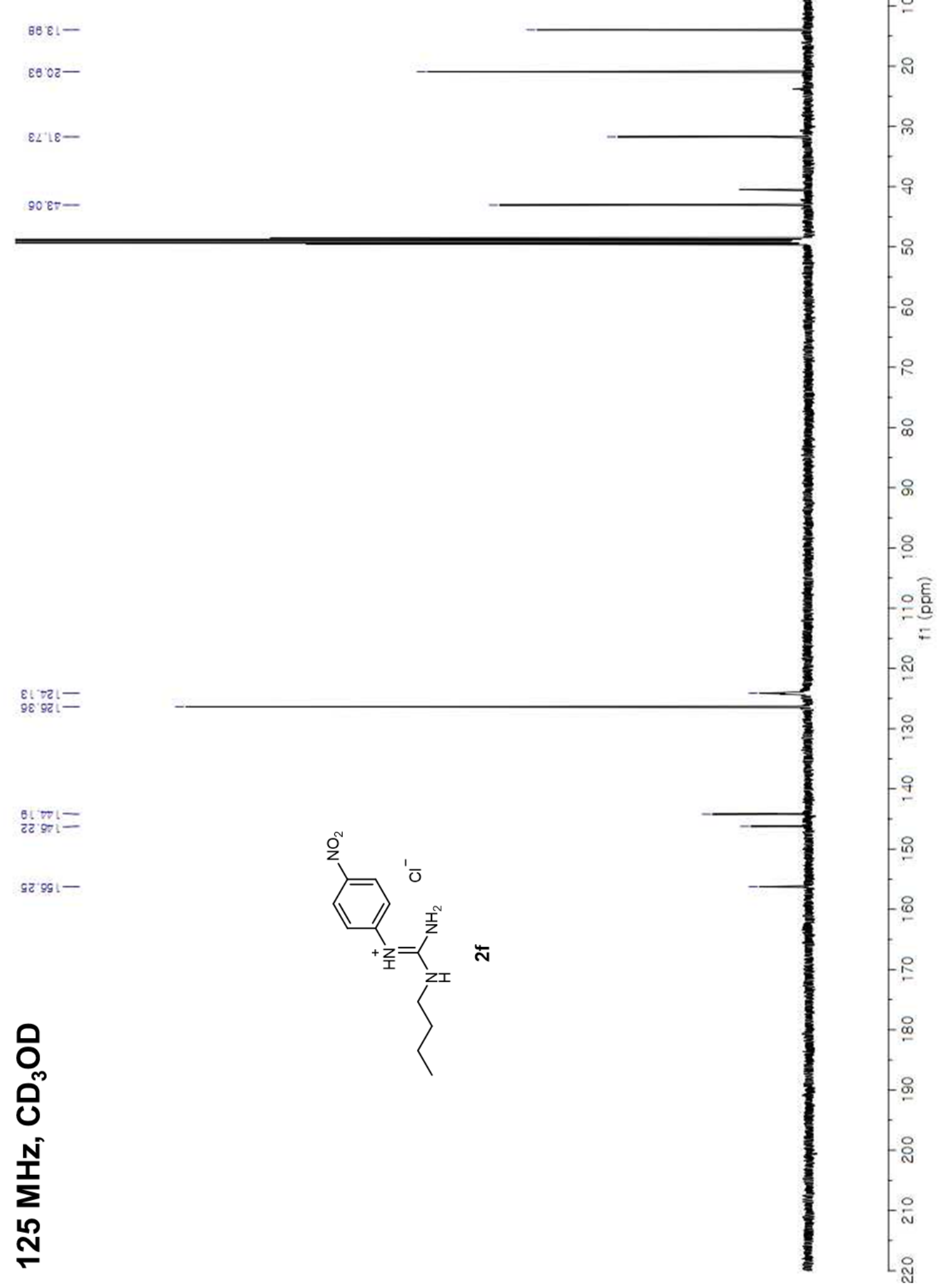




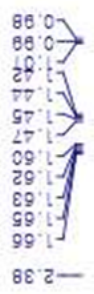

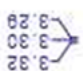

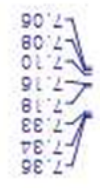

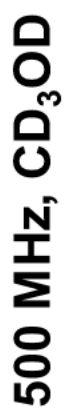

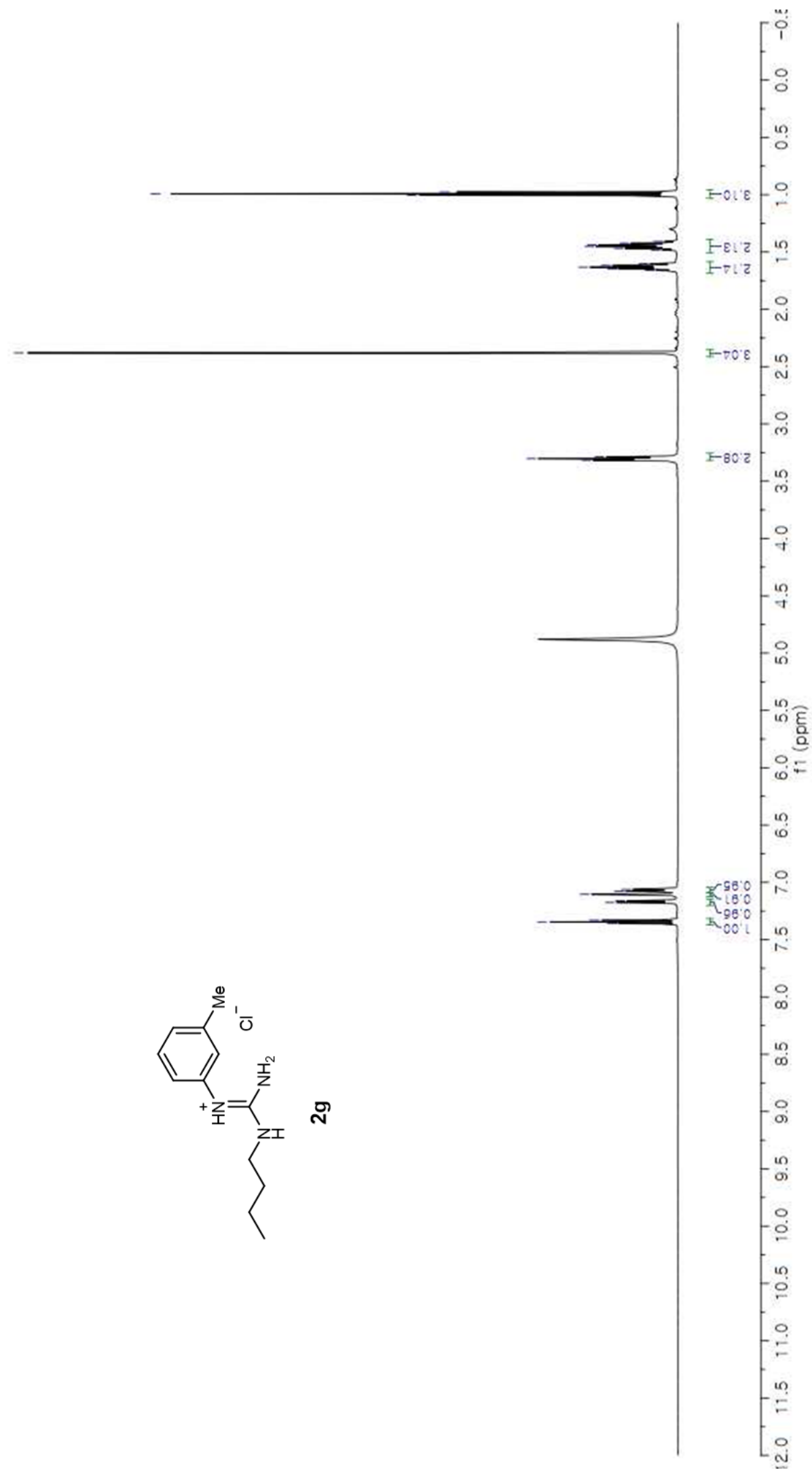




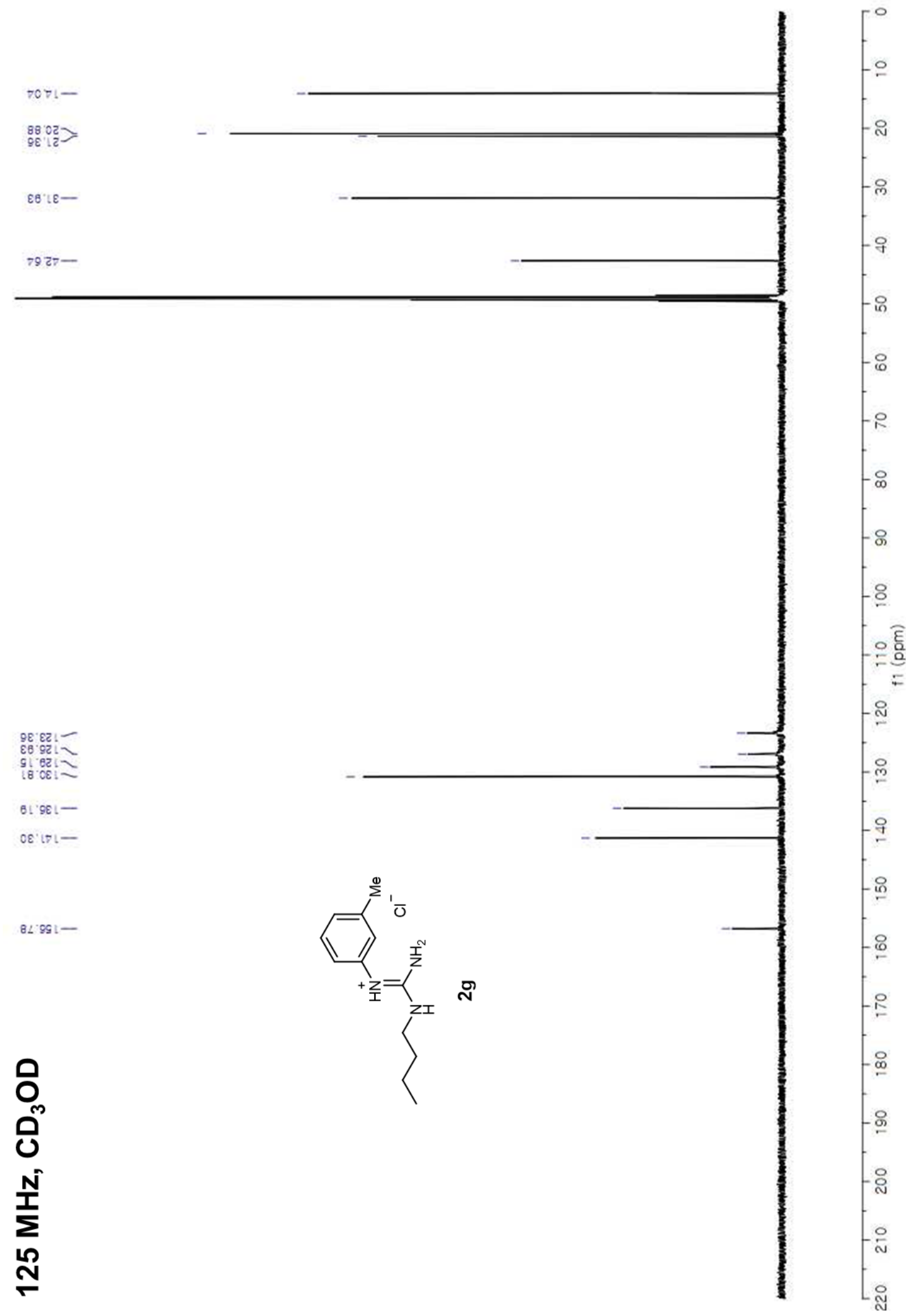




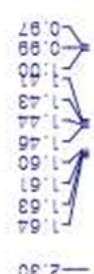

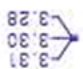

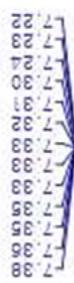

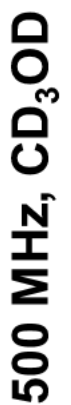

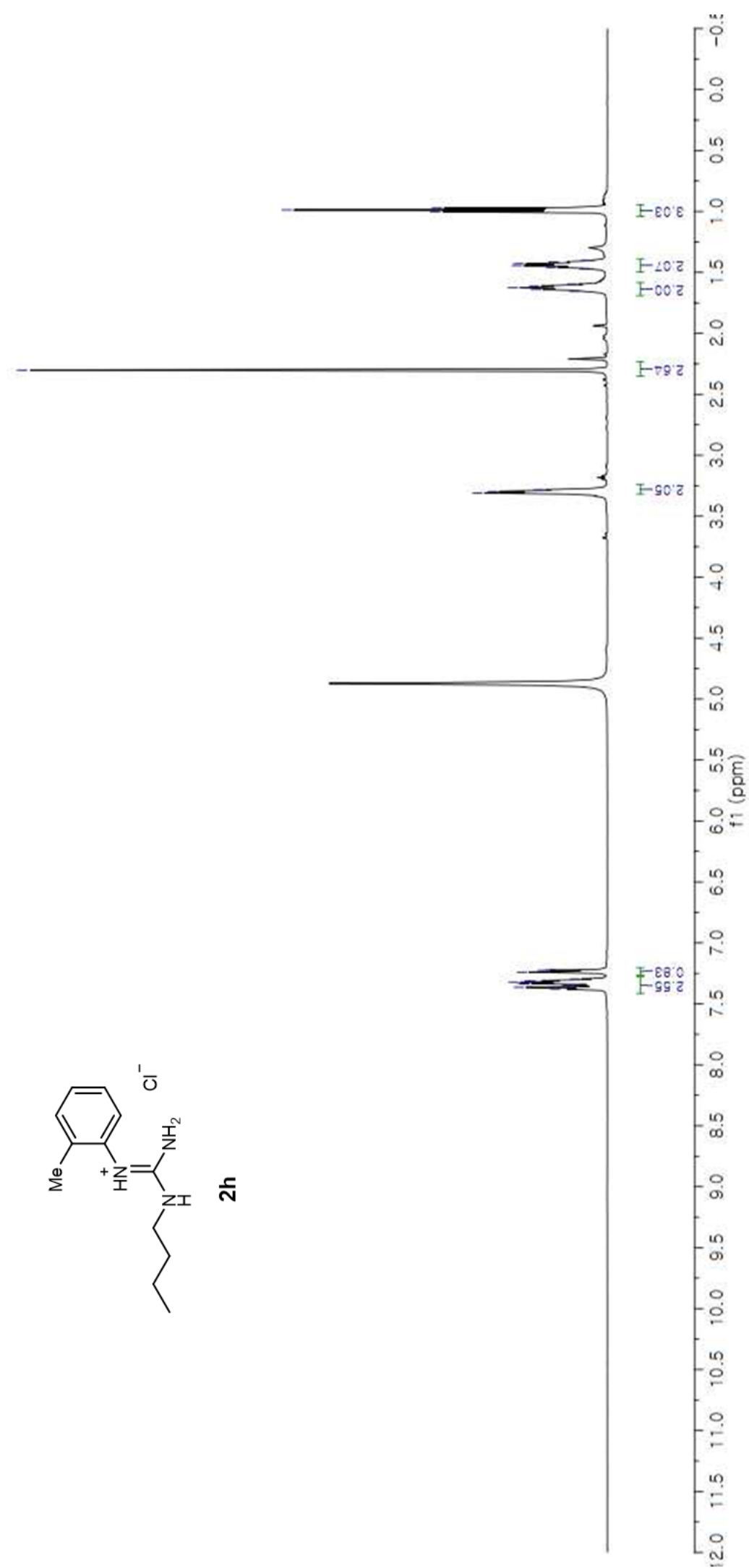




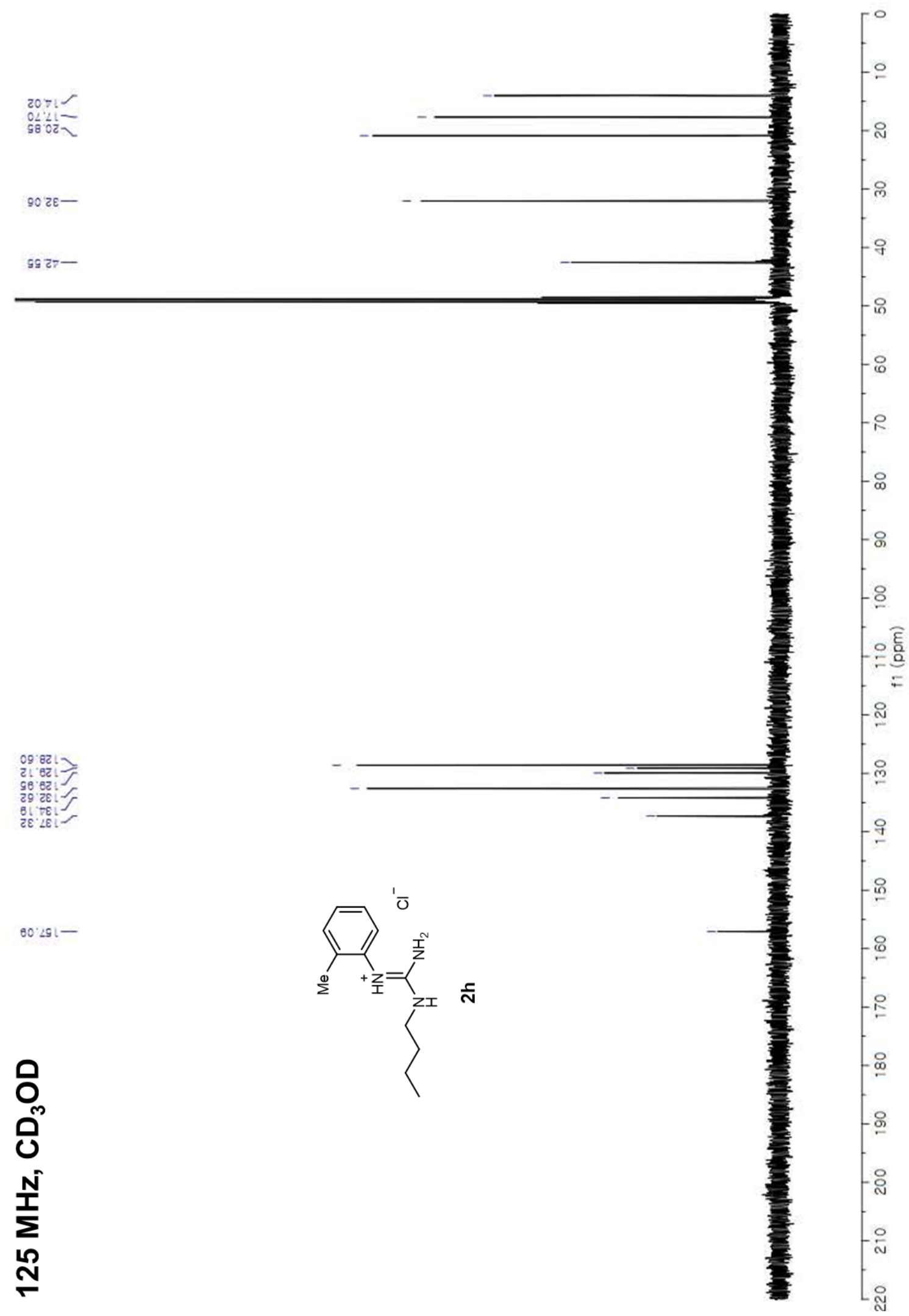




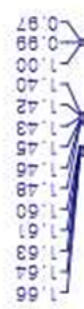

ere

of

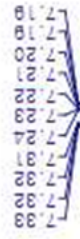

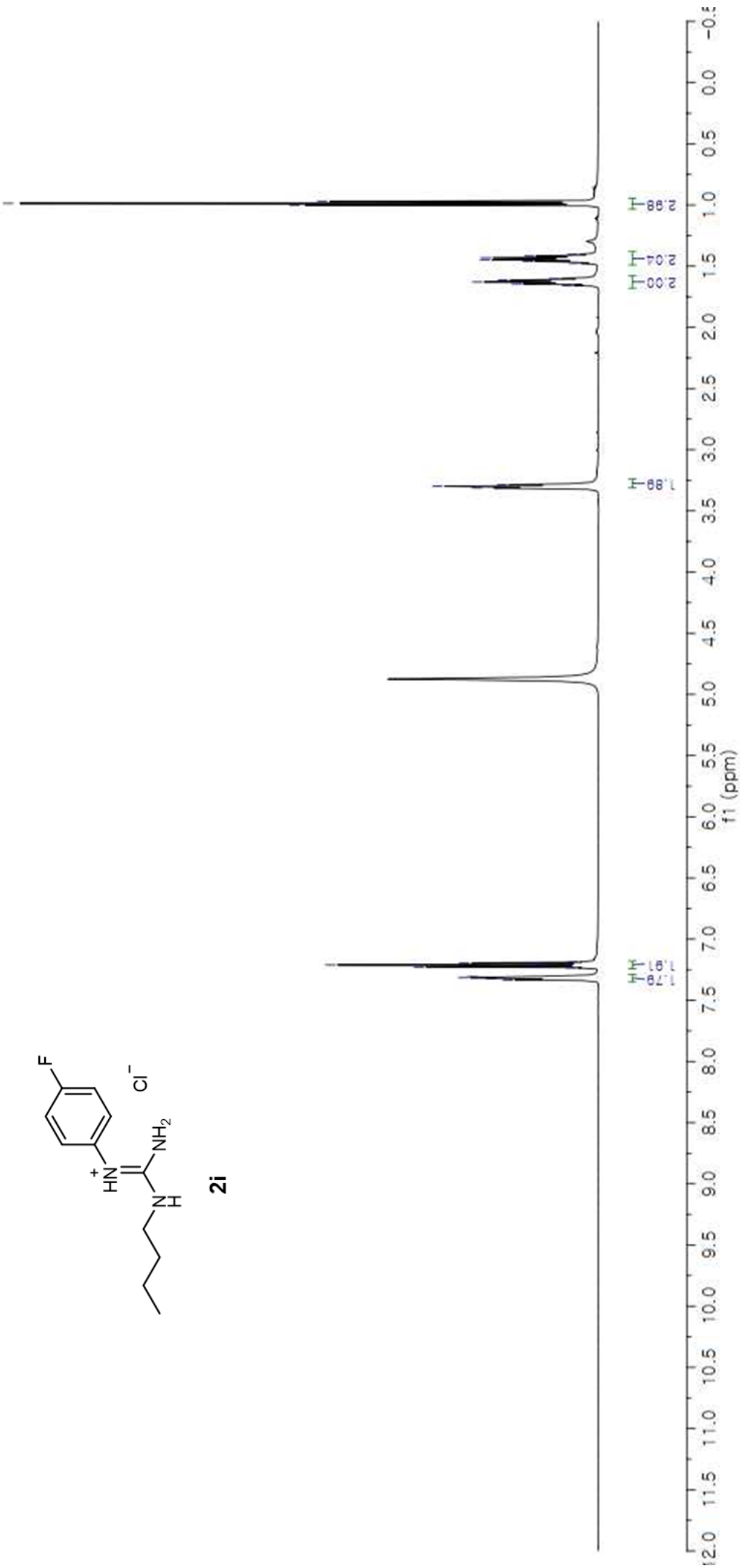




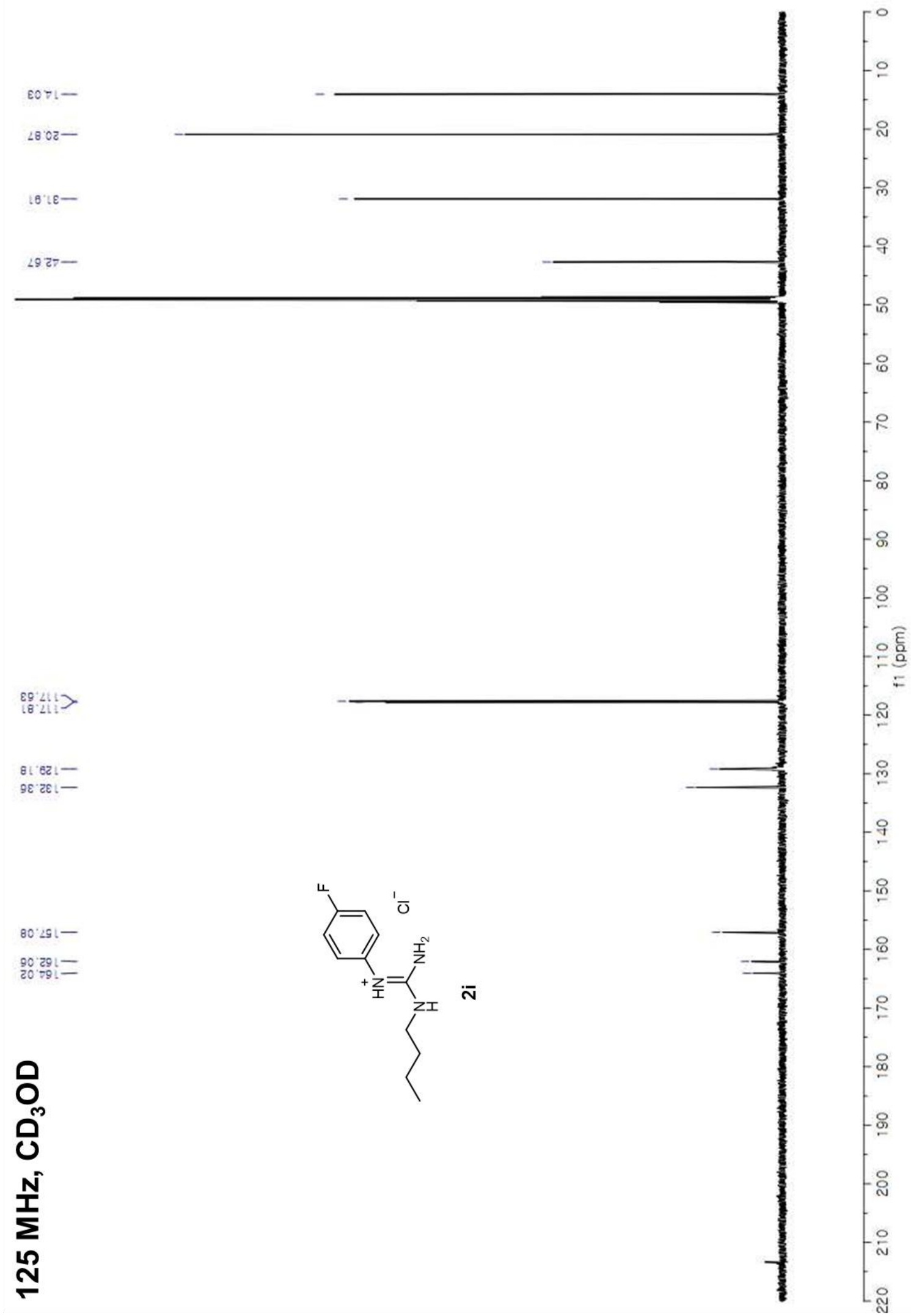




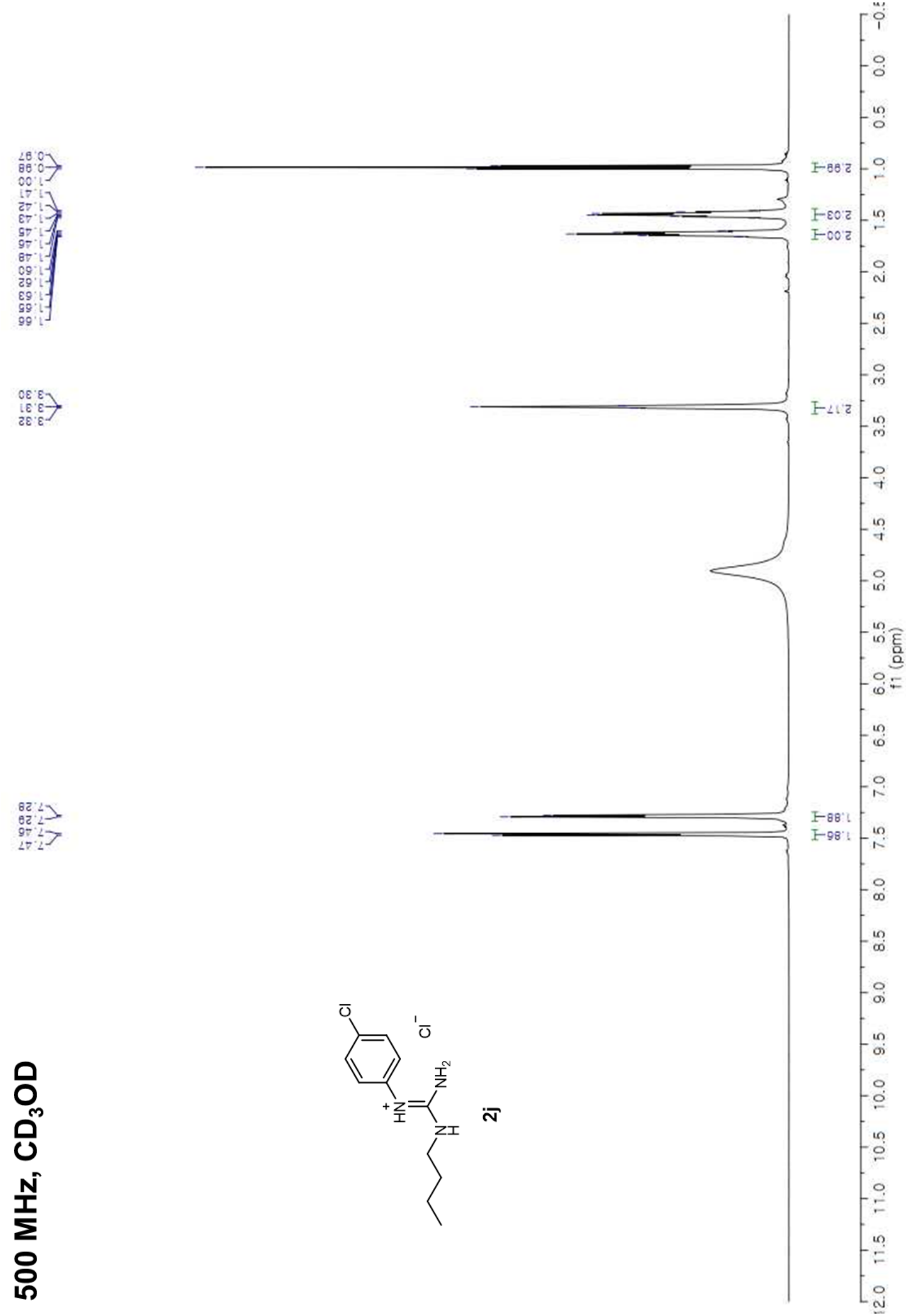




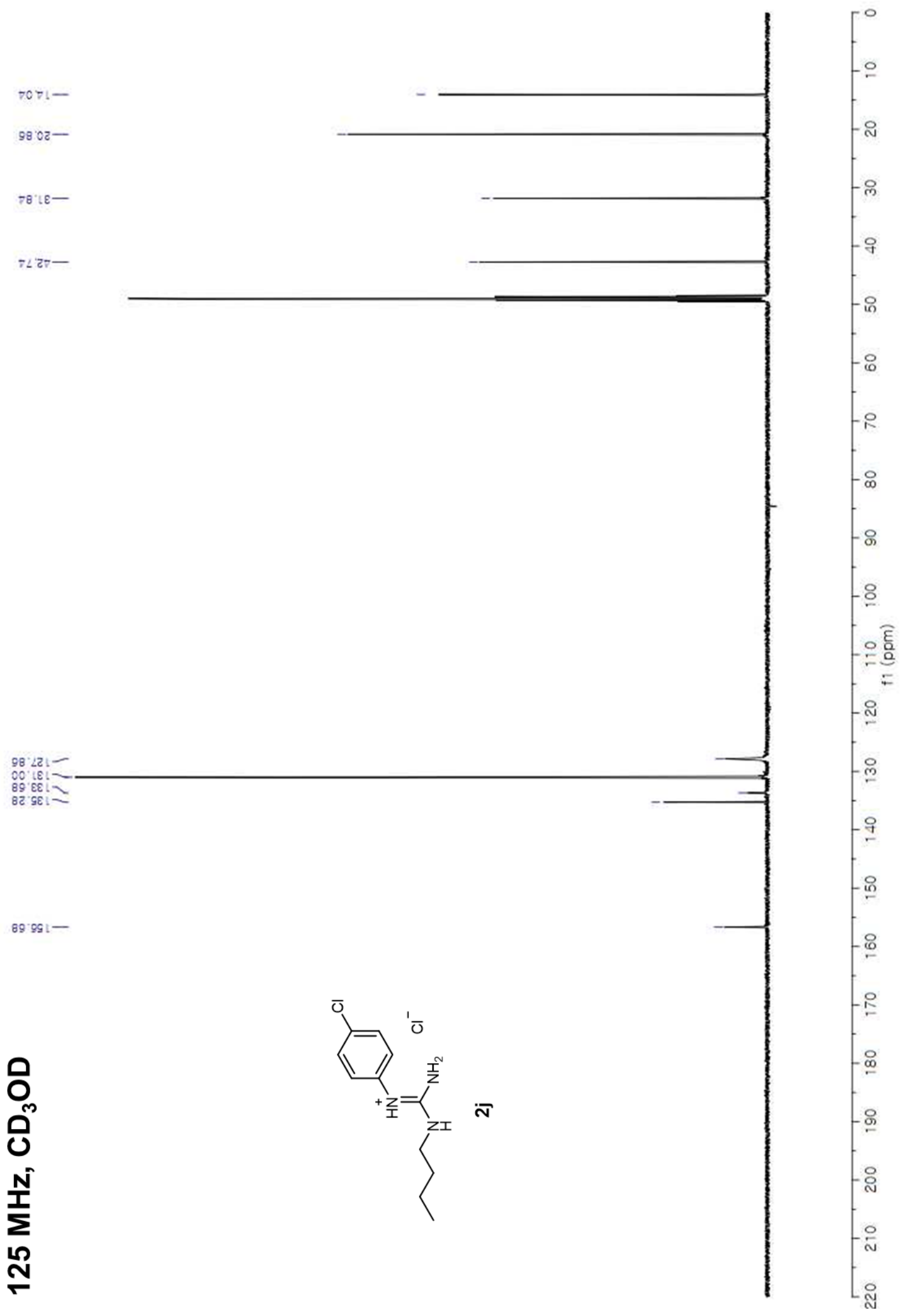




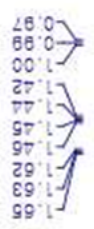

${ }_{0}^{\circ \varepsilon} \varepsilon>>$
${ }_{\varepsilon} \varepsilon$

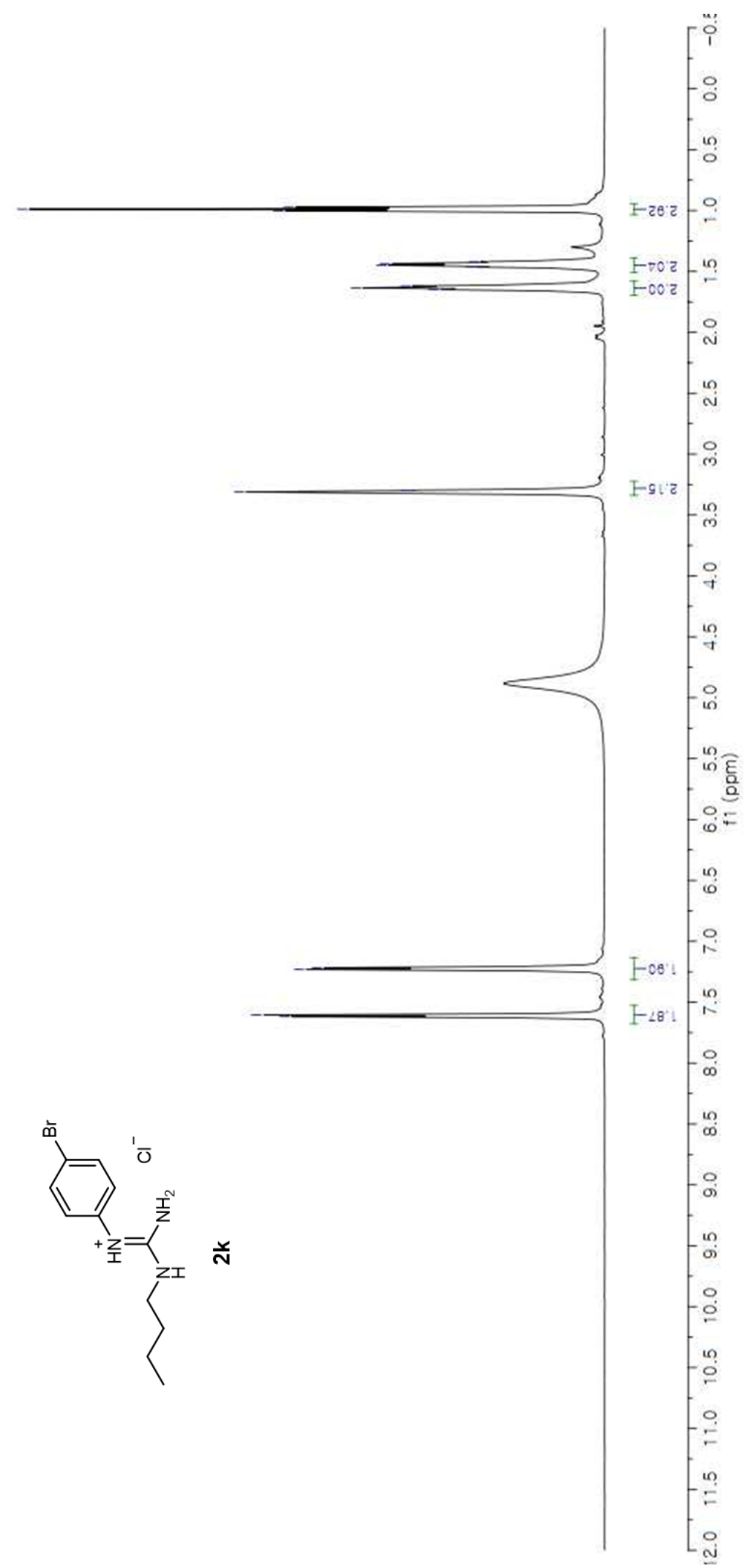

${ }_{22}^{22}: 2>$

${ }_{29} 9.2>$

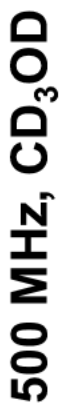




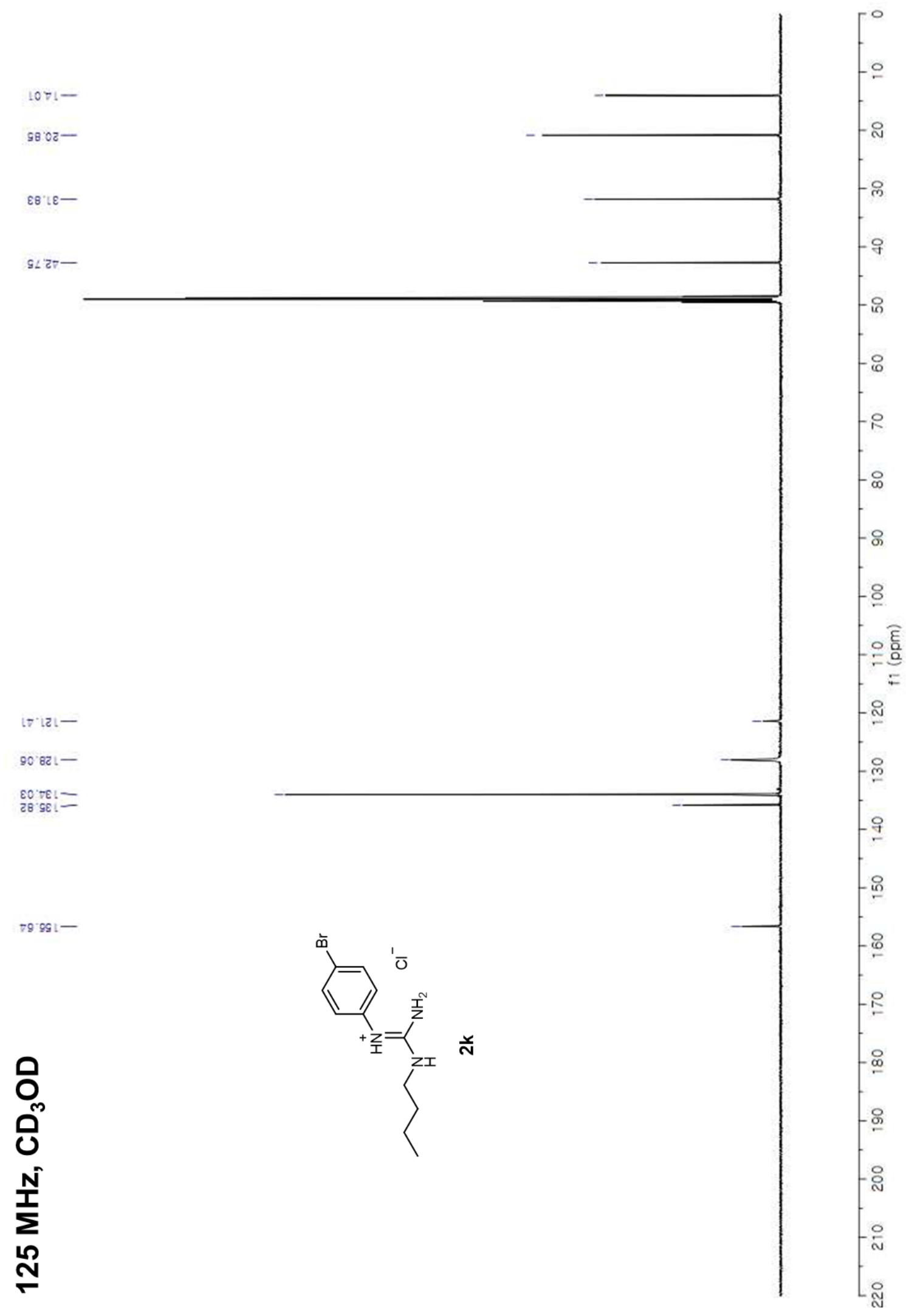




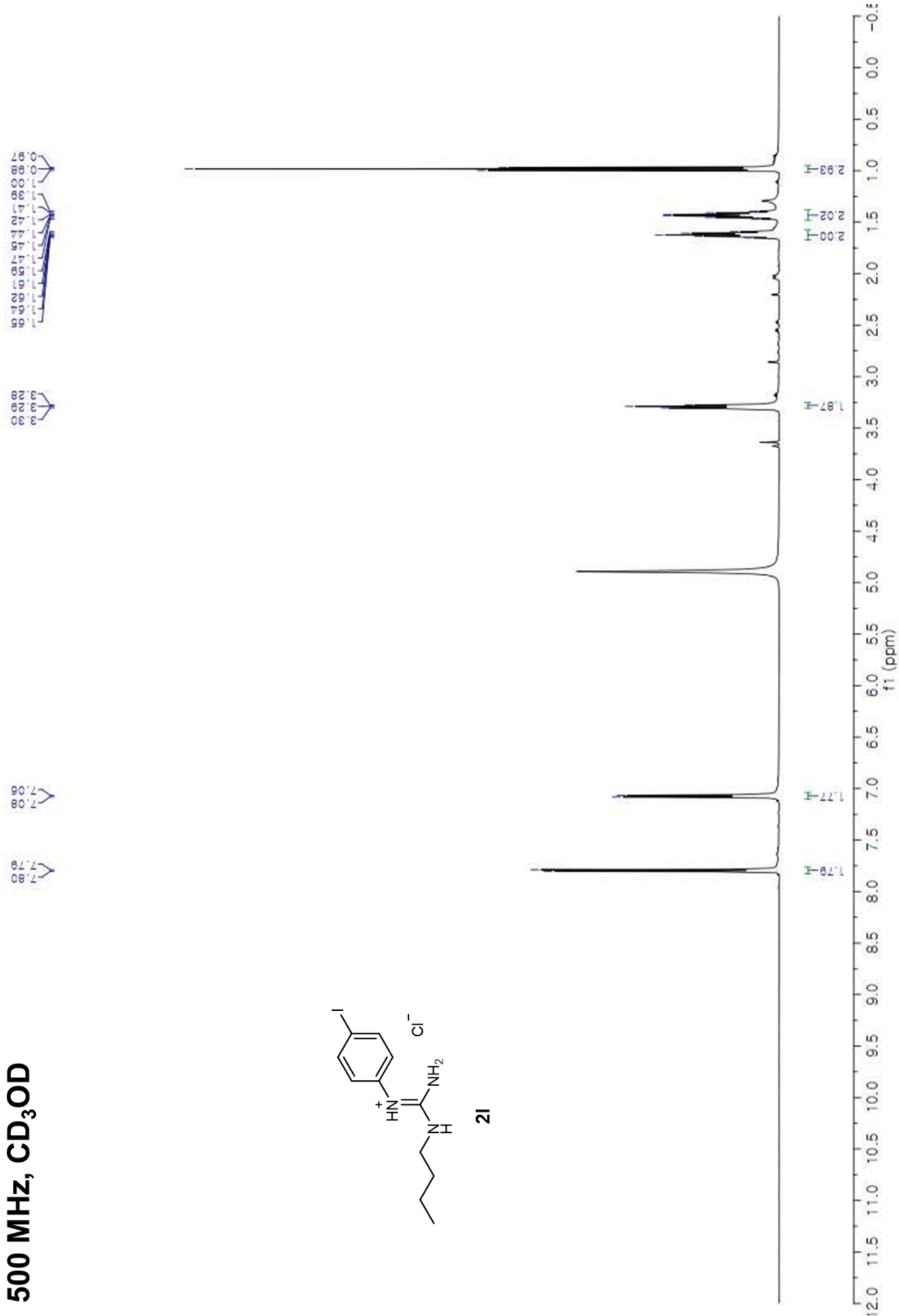




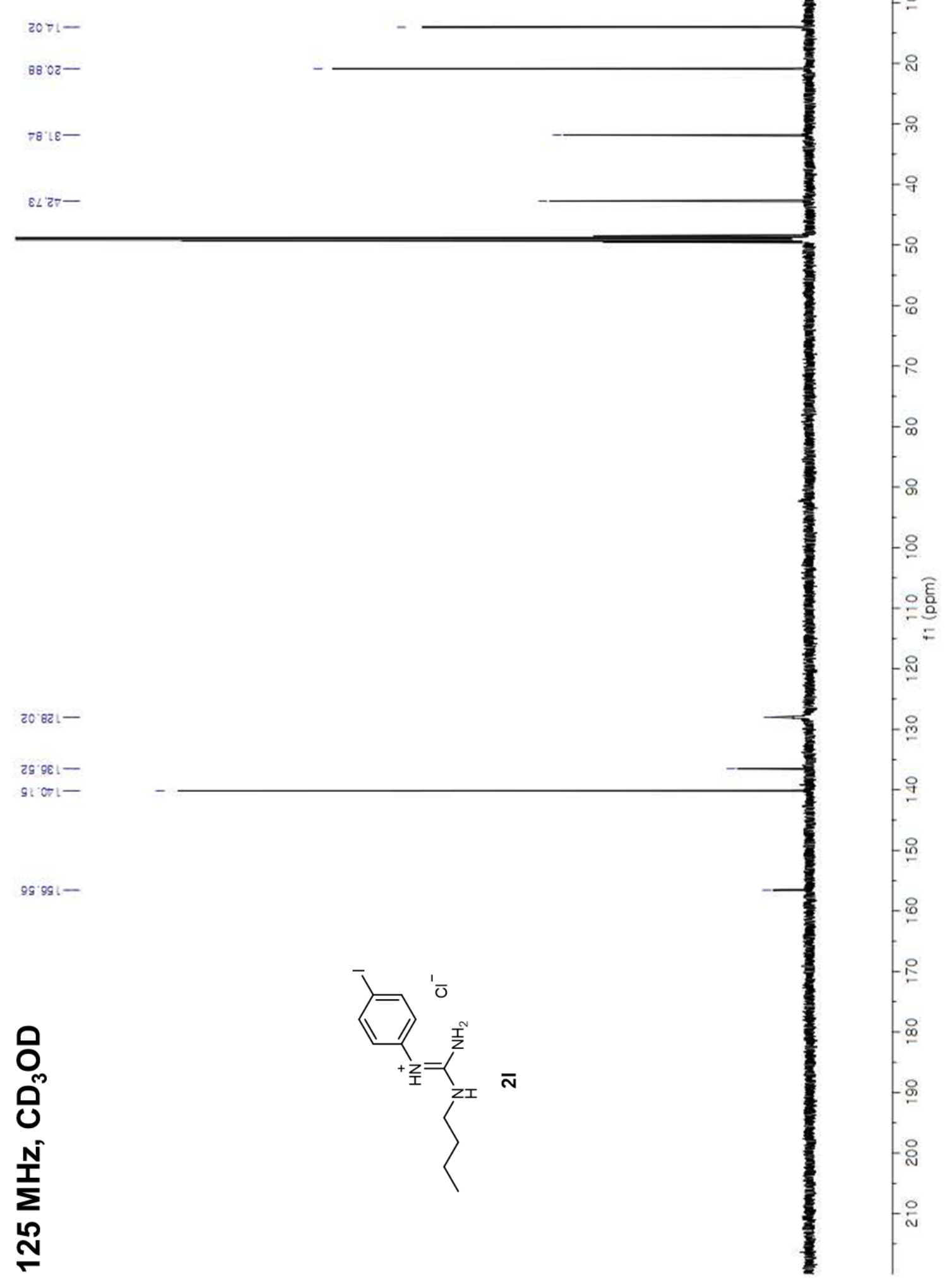




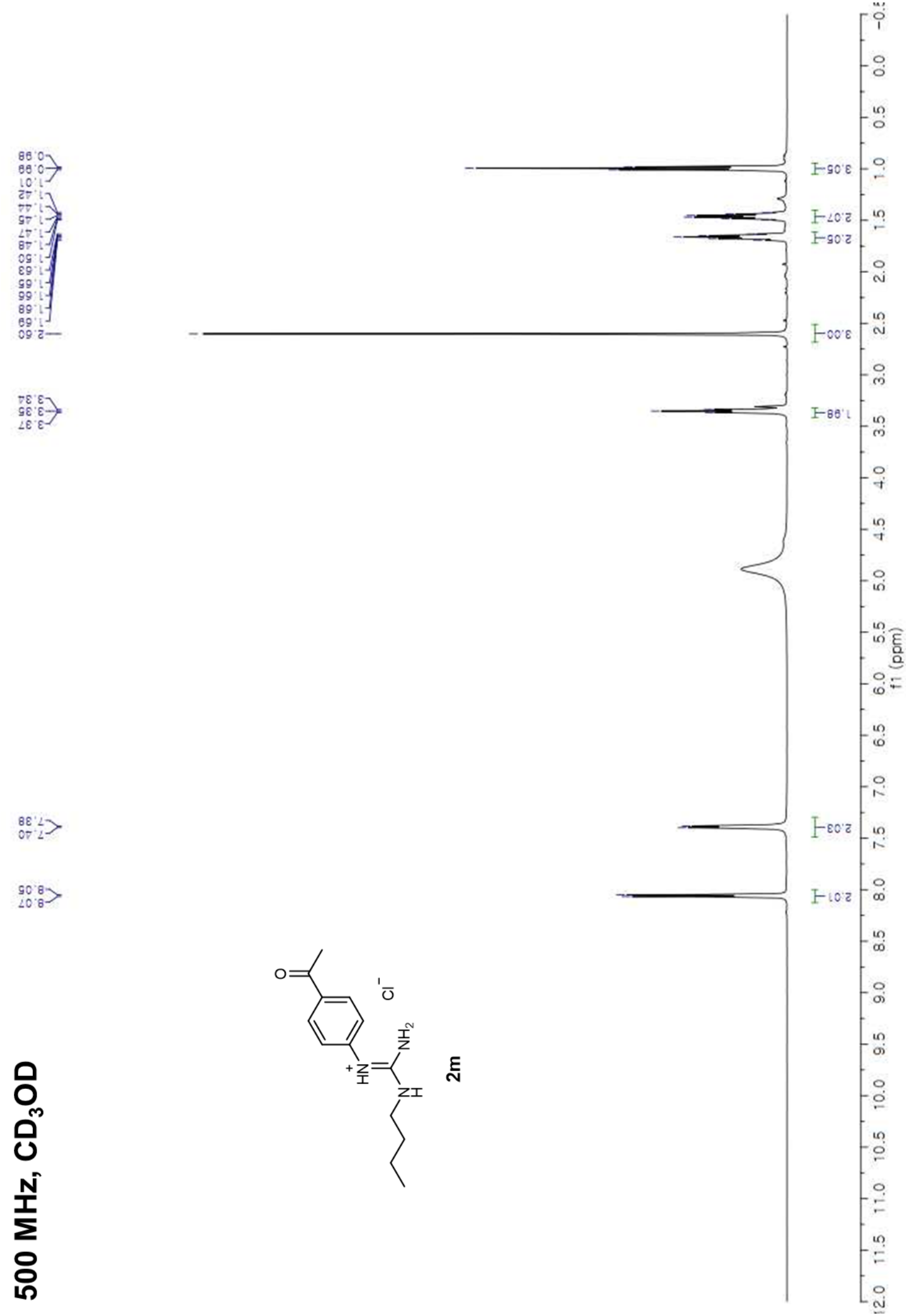




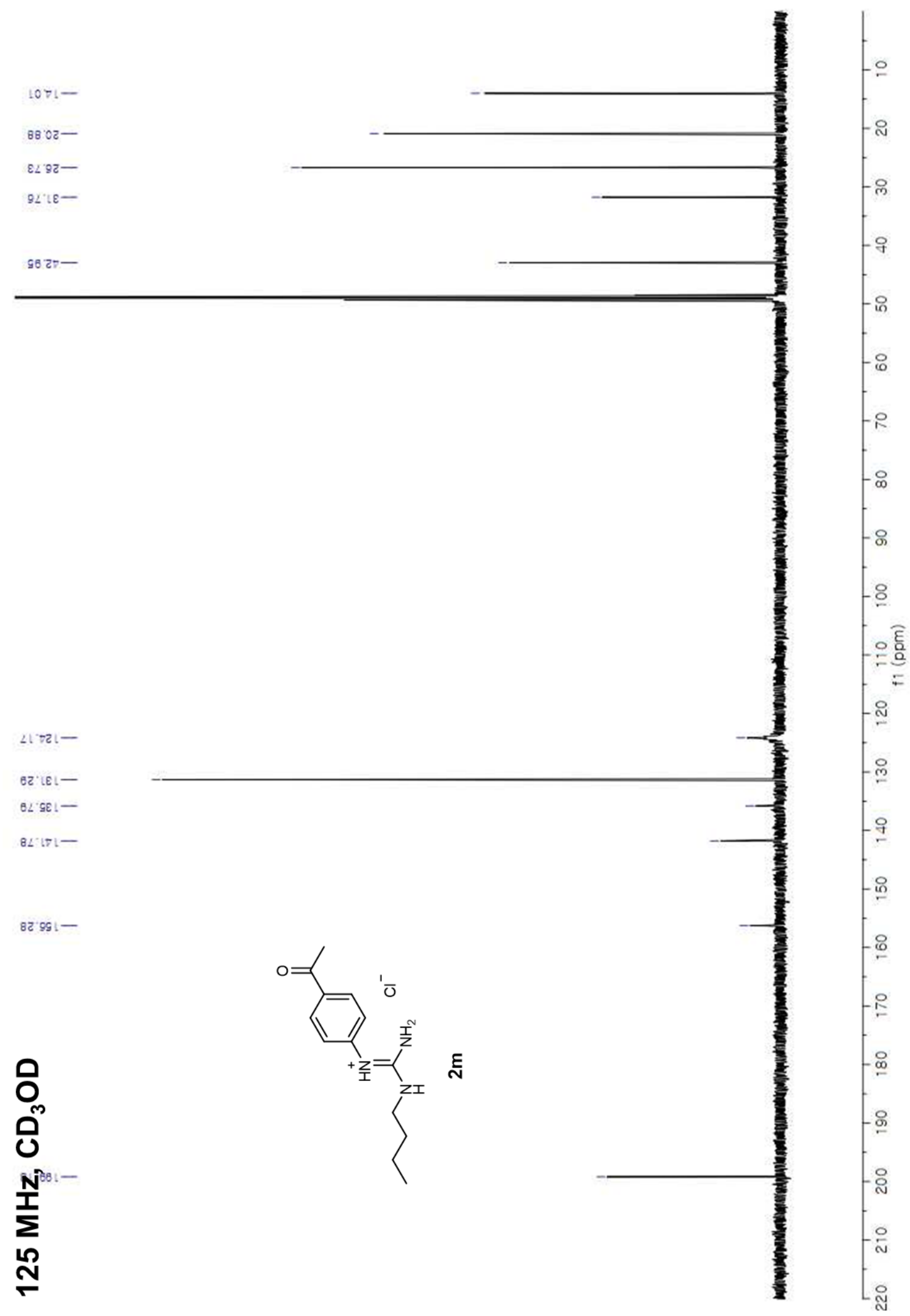



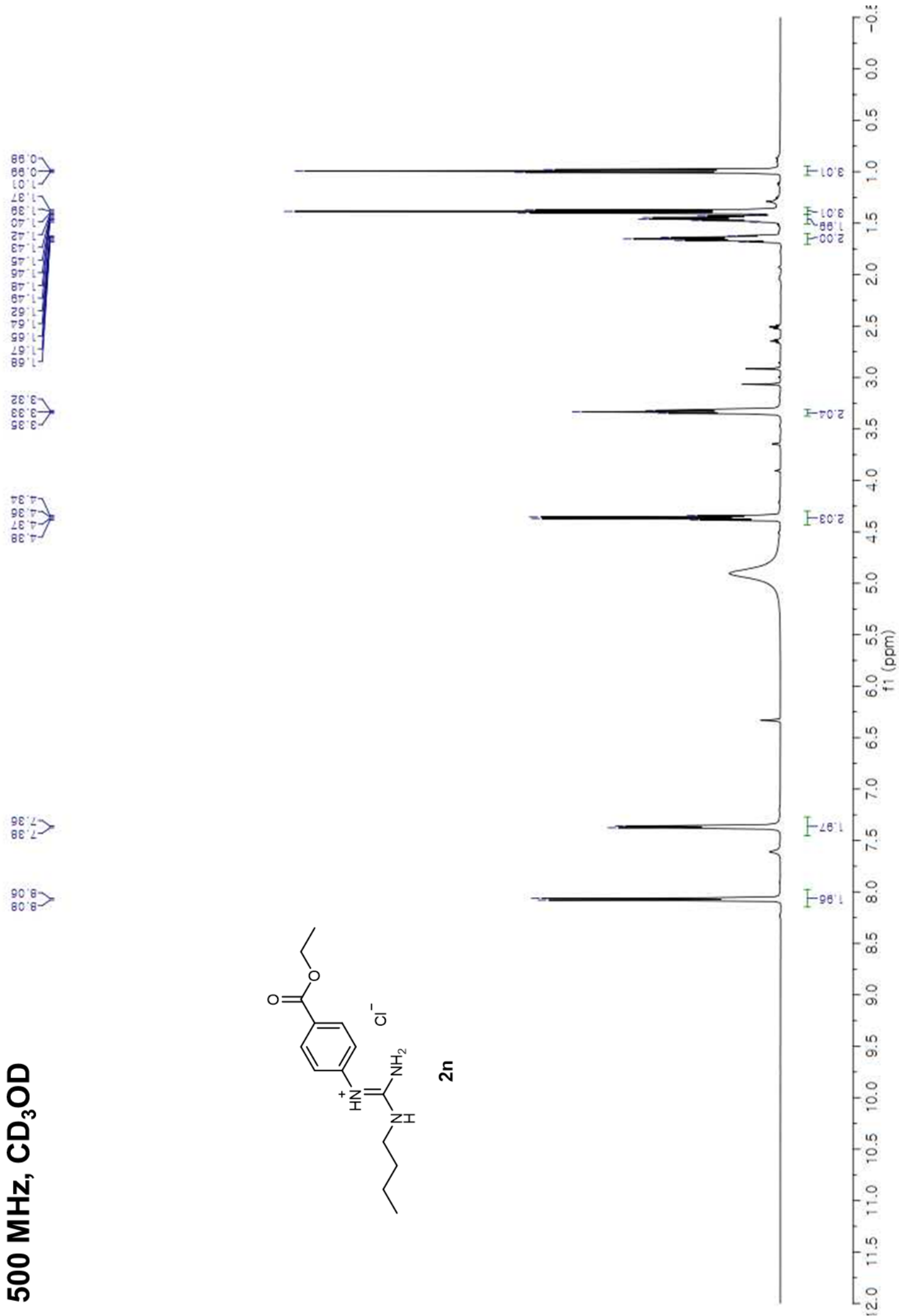


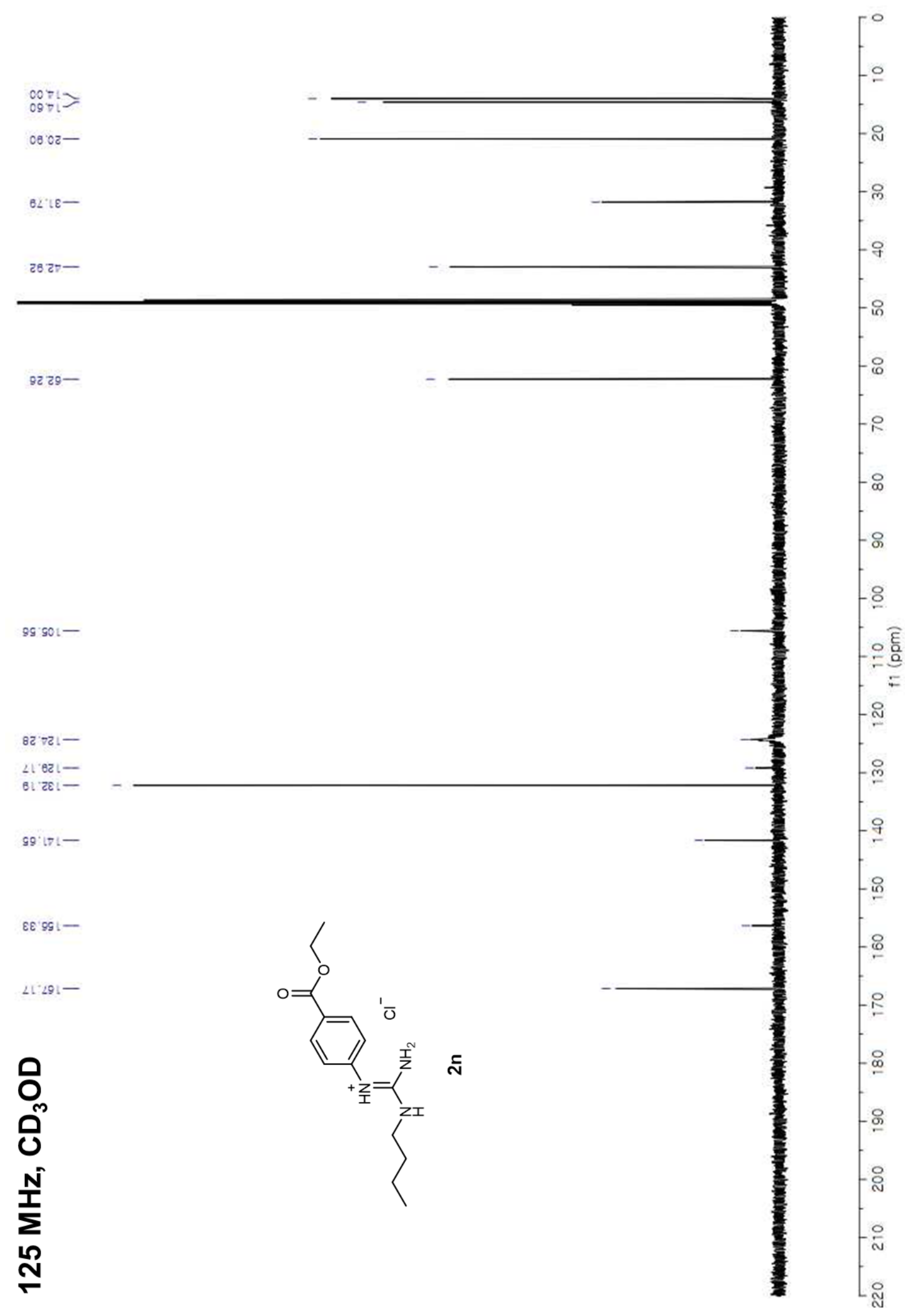



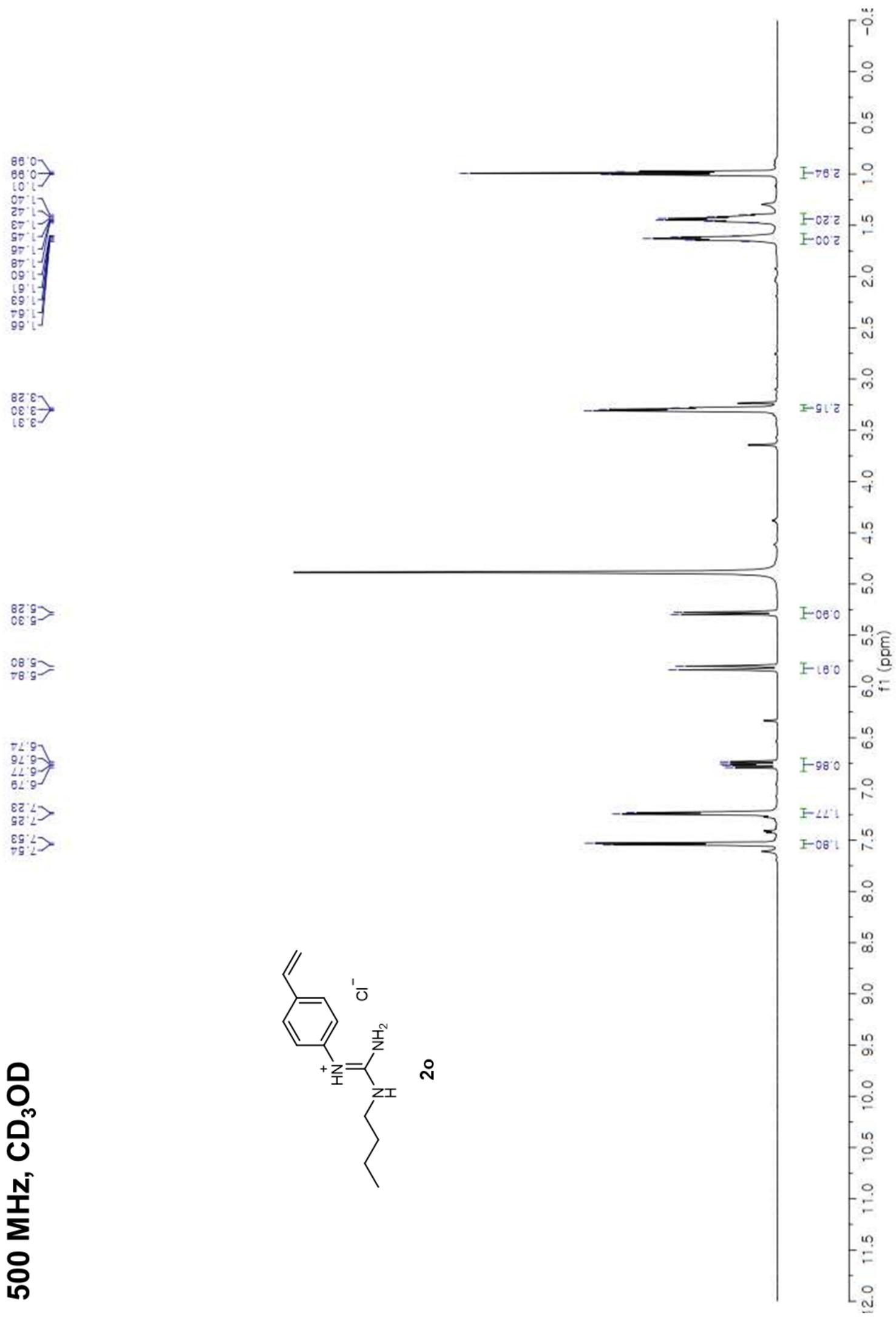


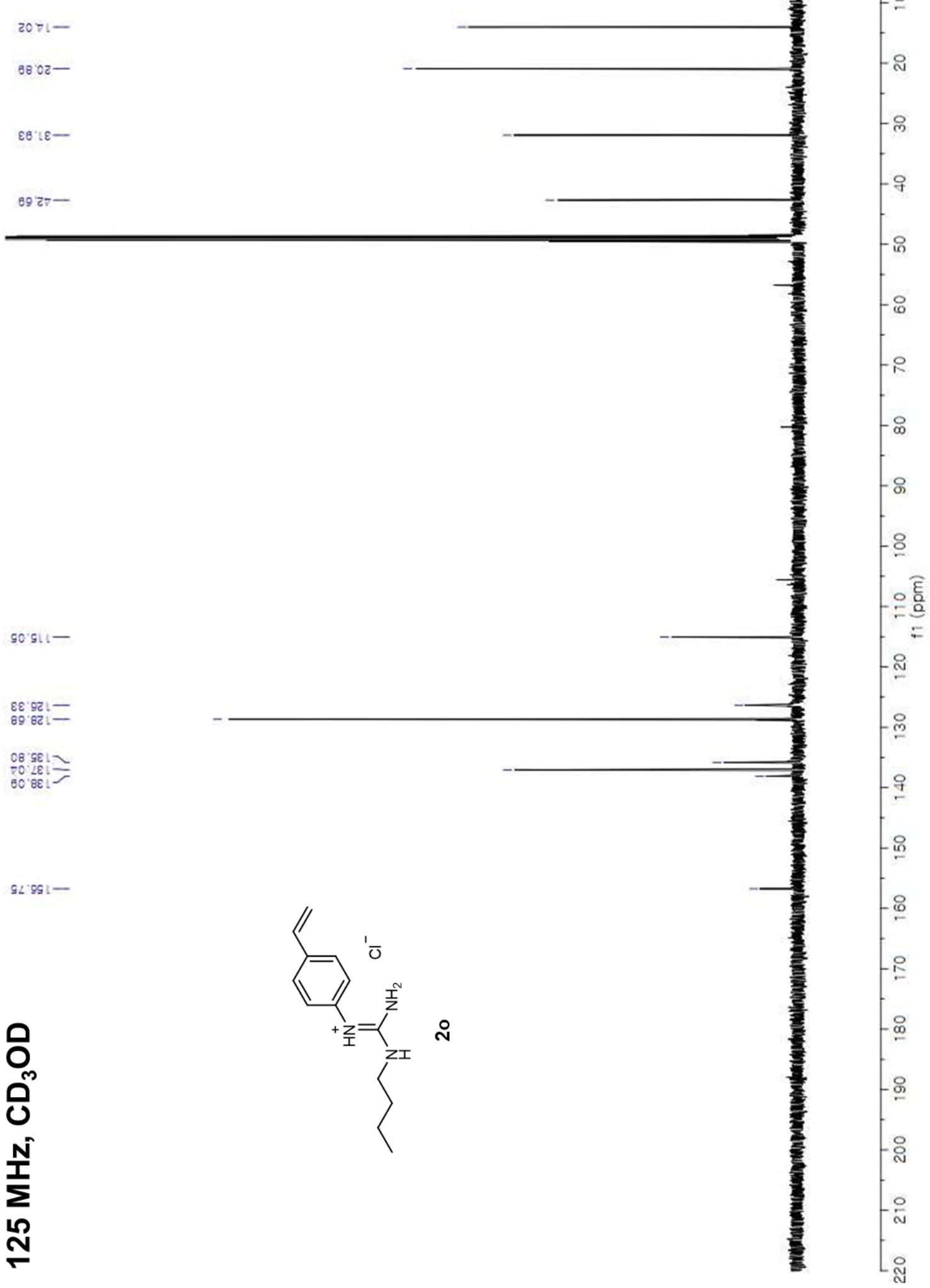



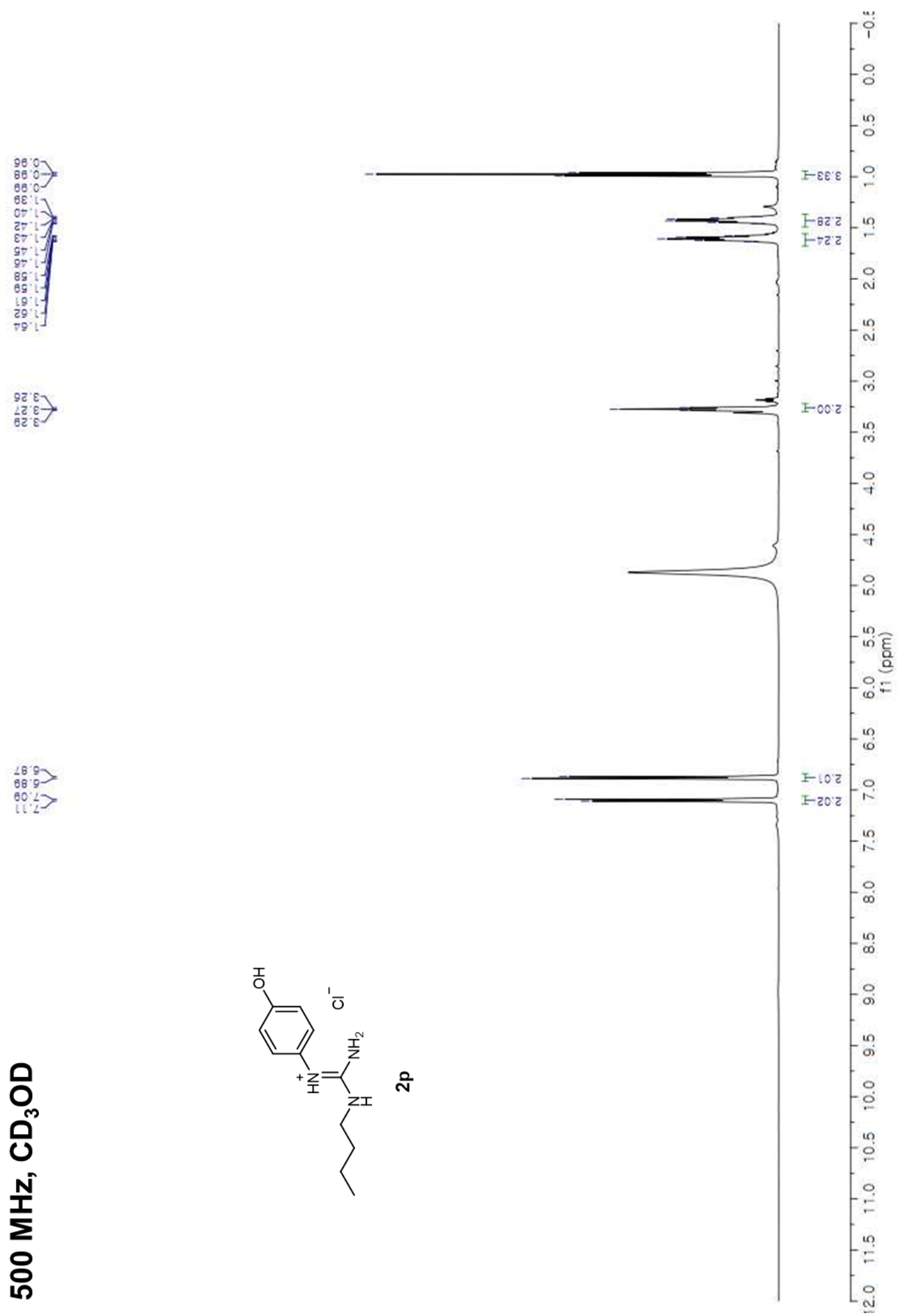


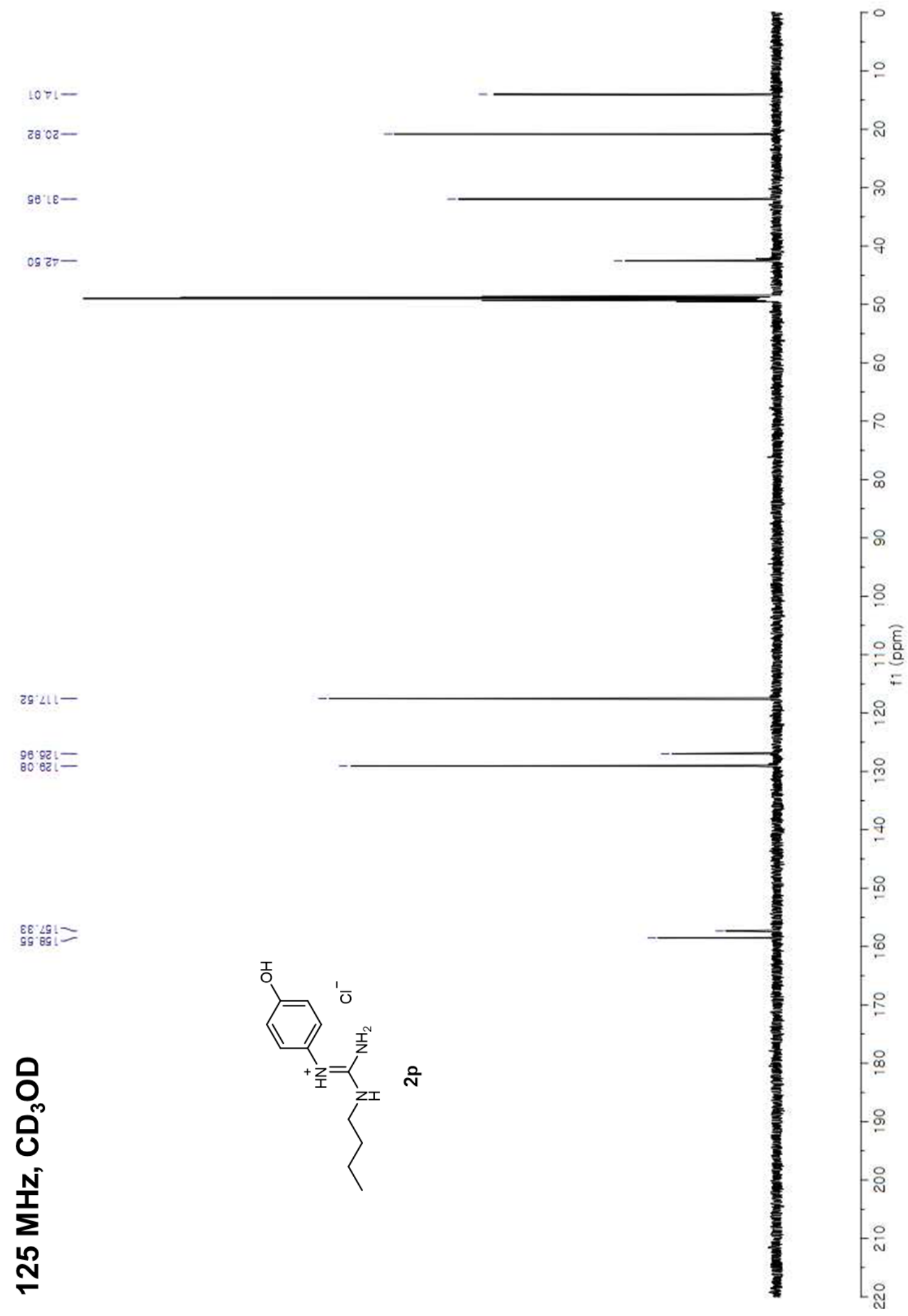




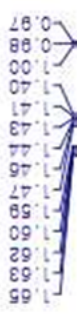

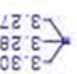

$80 \%$

929
22.97
82.97

$82.9-5$

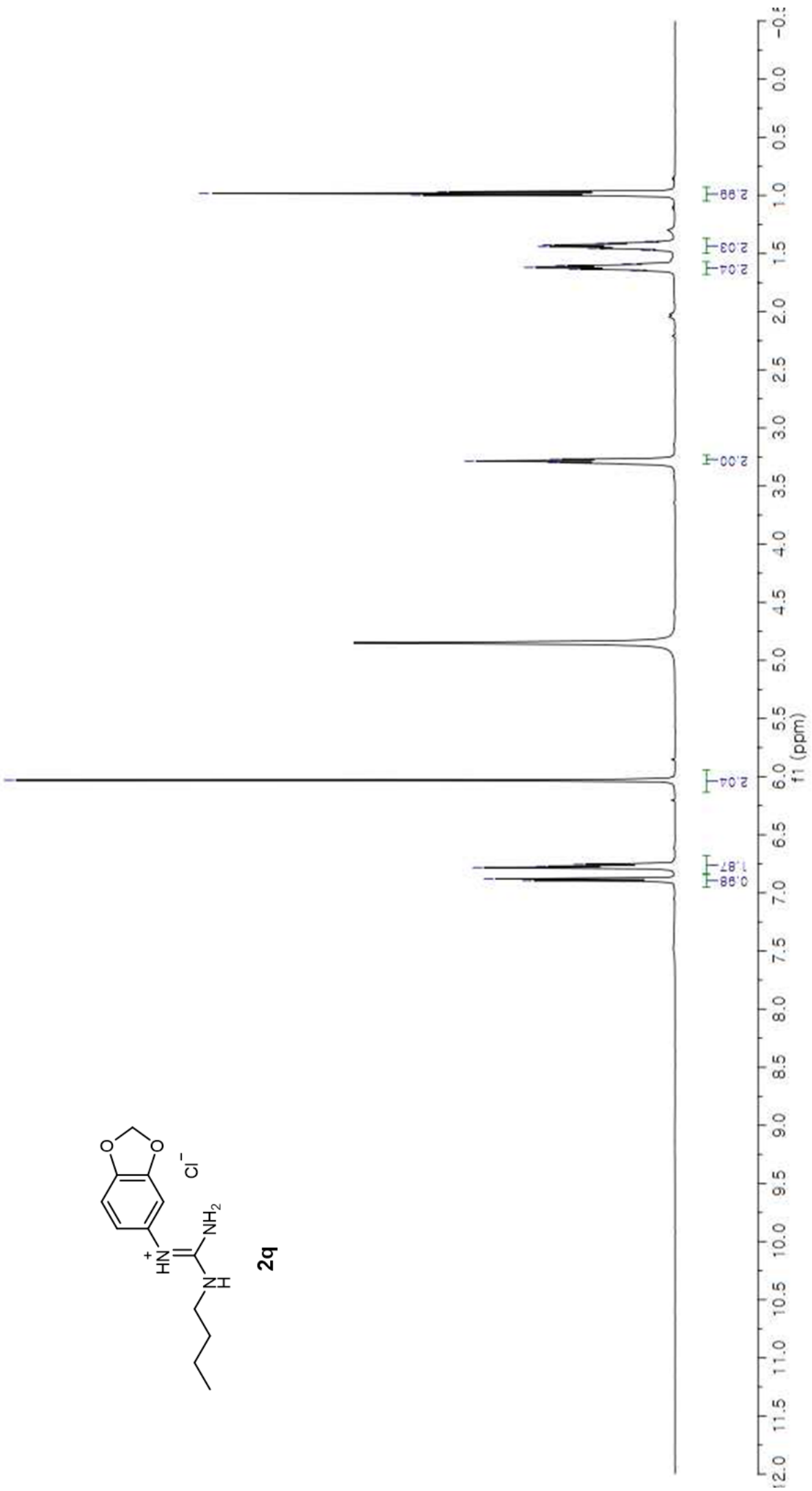




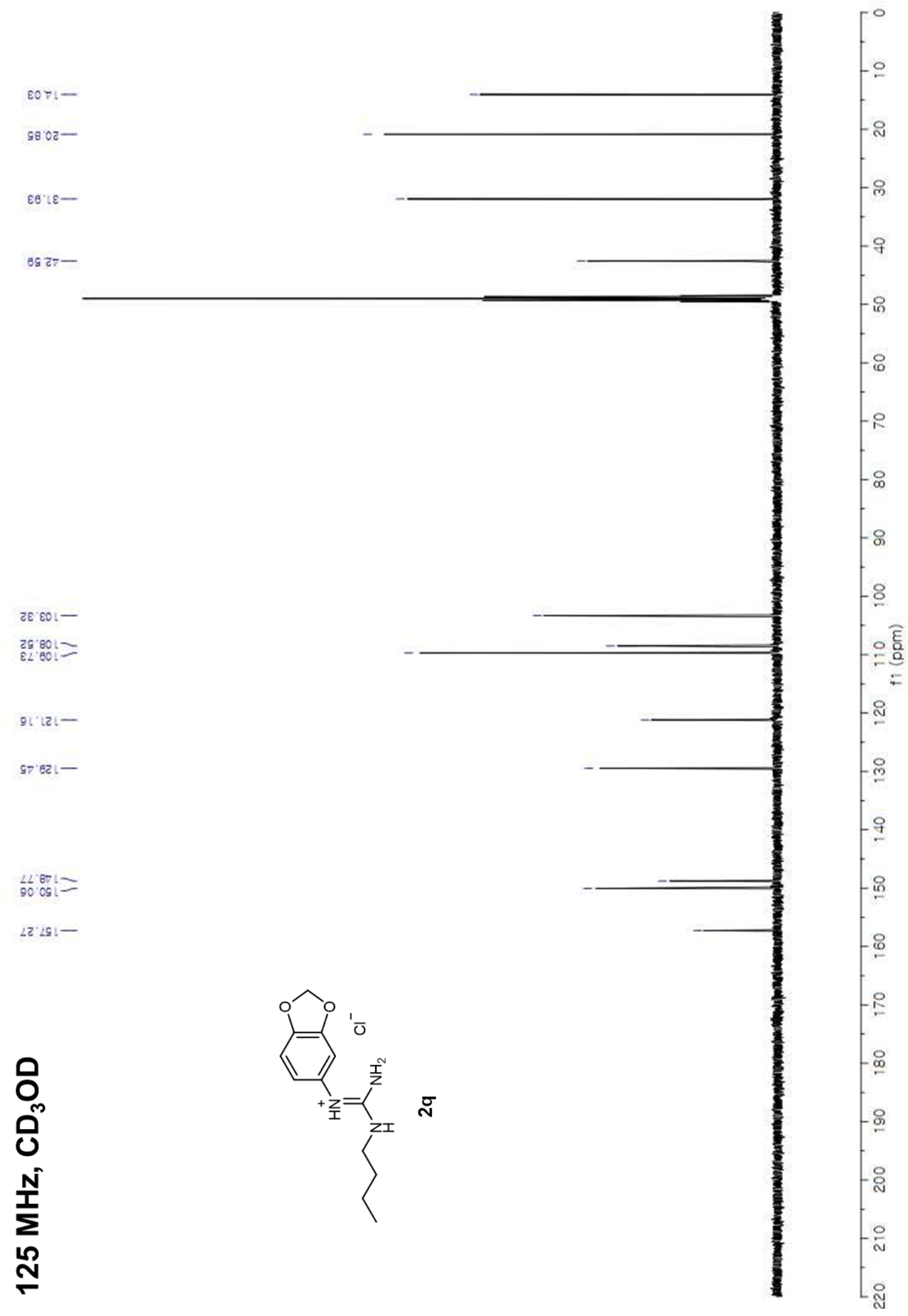



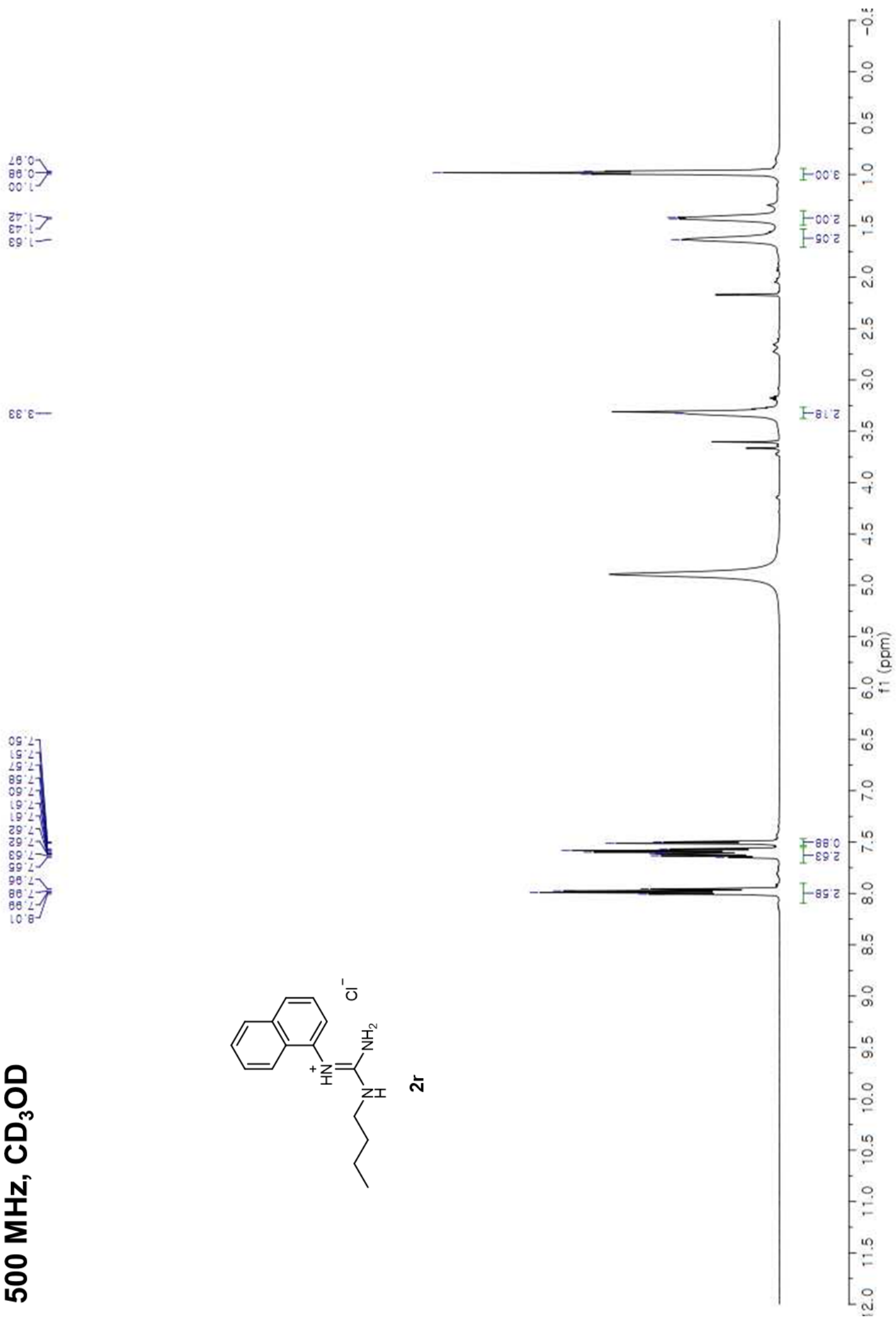

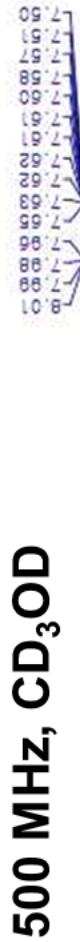




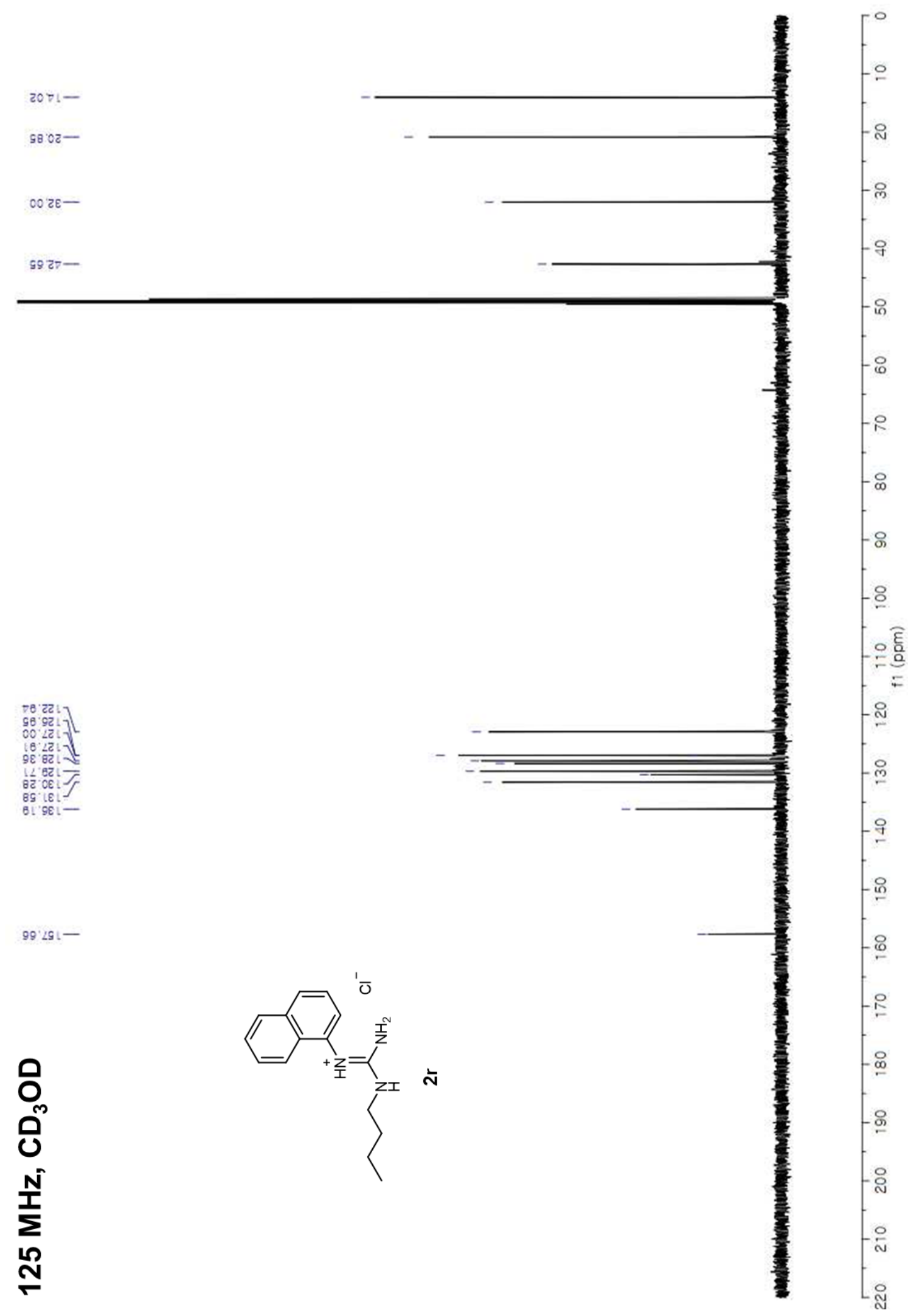




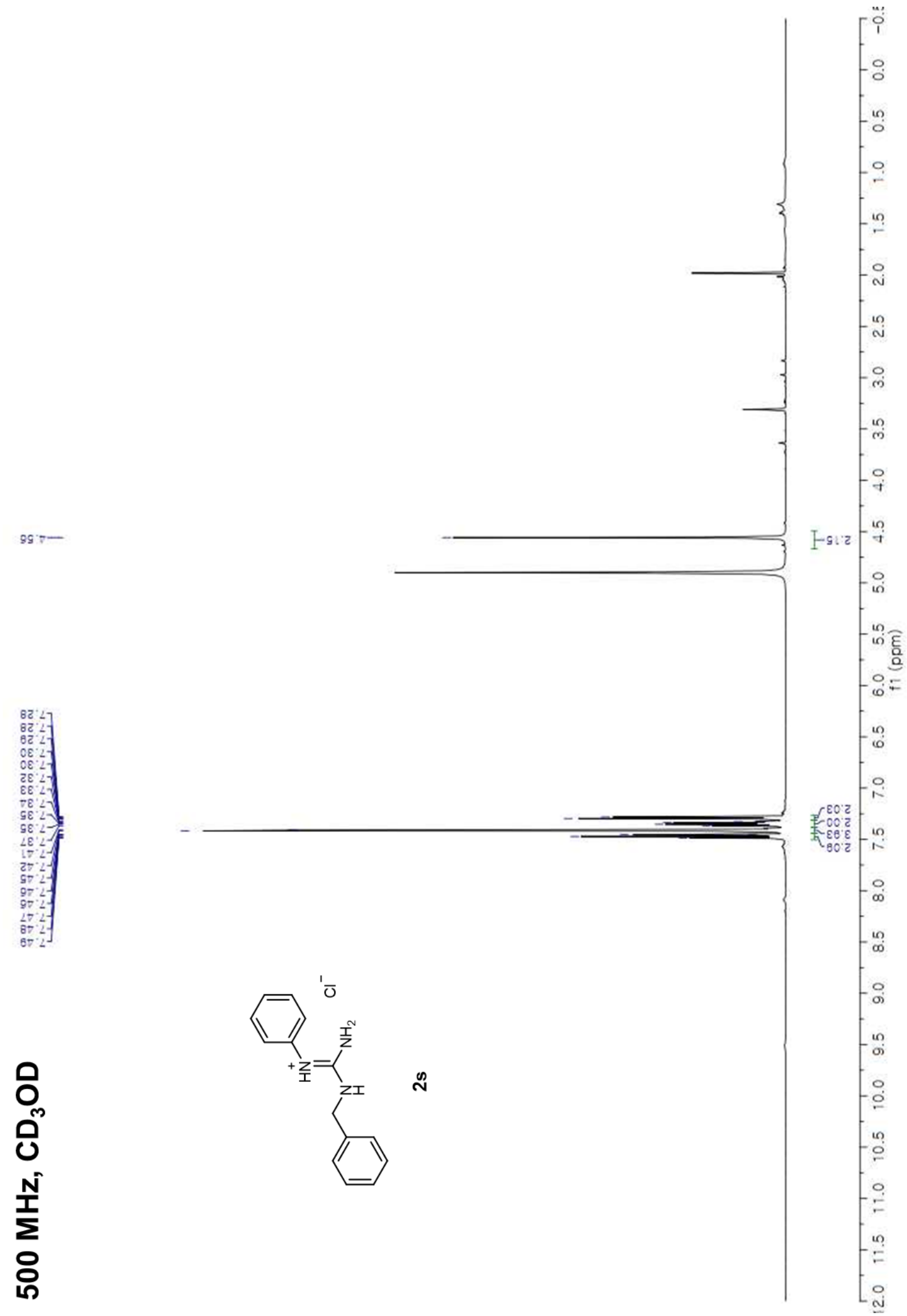




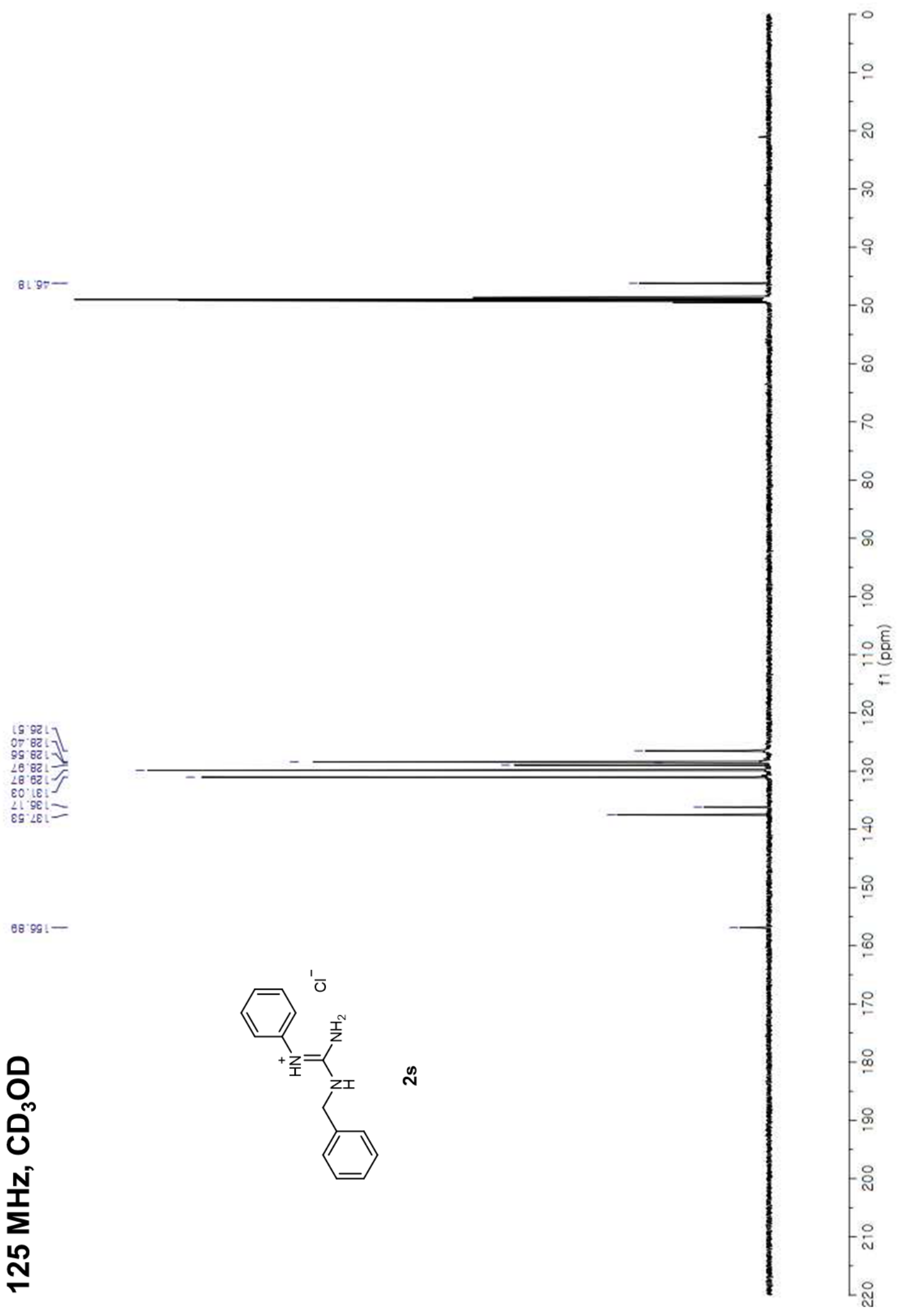




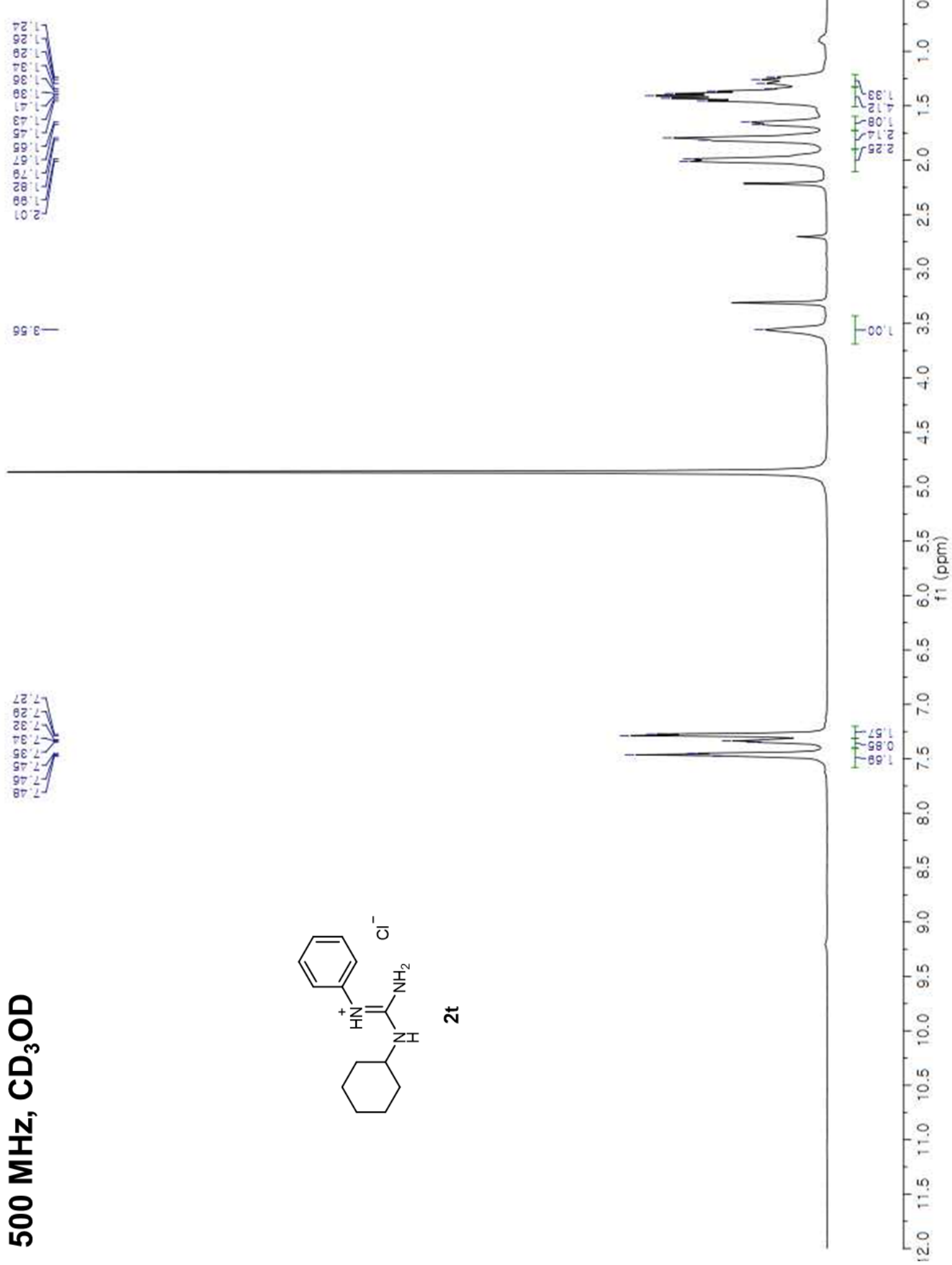




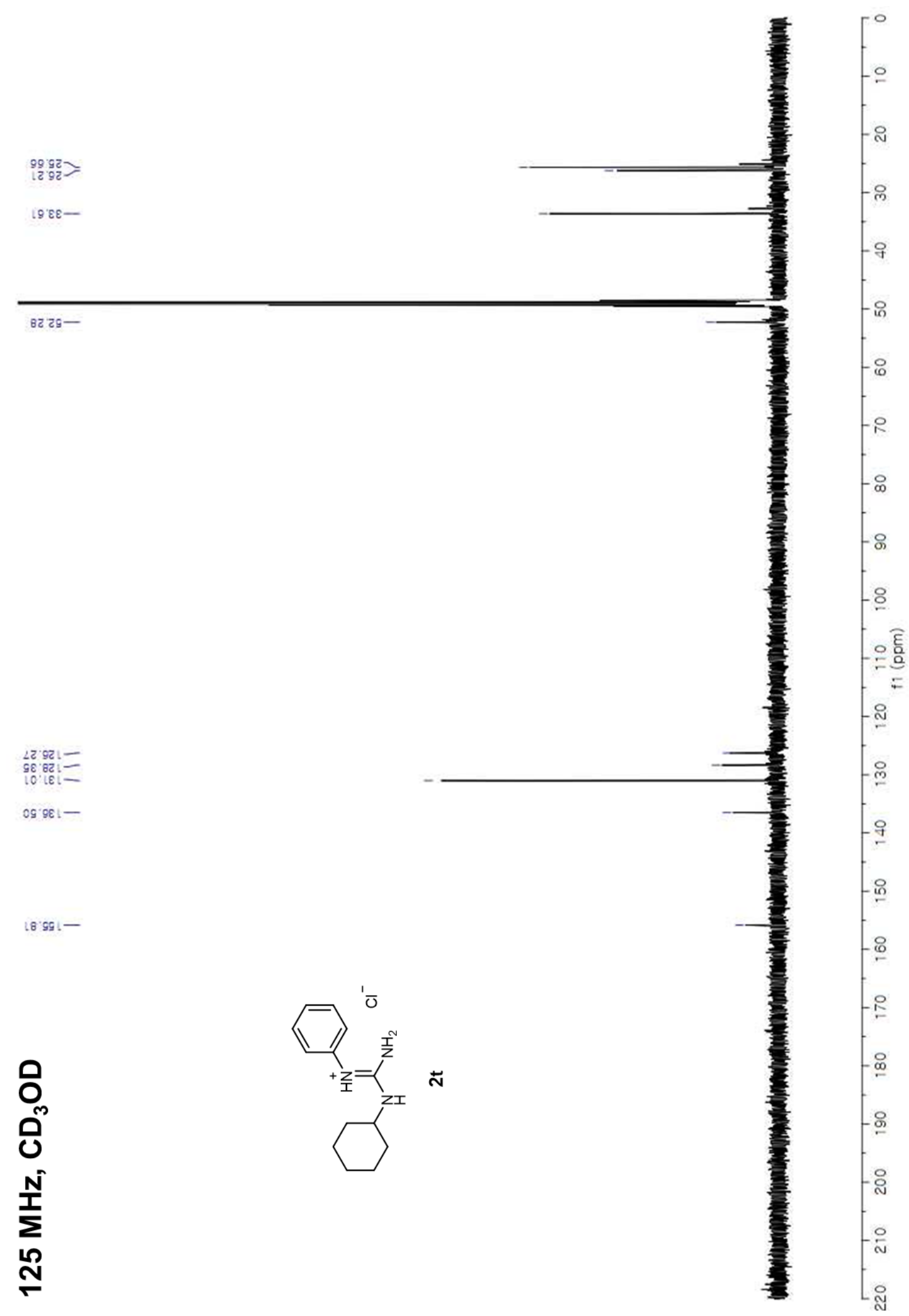




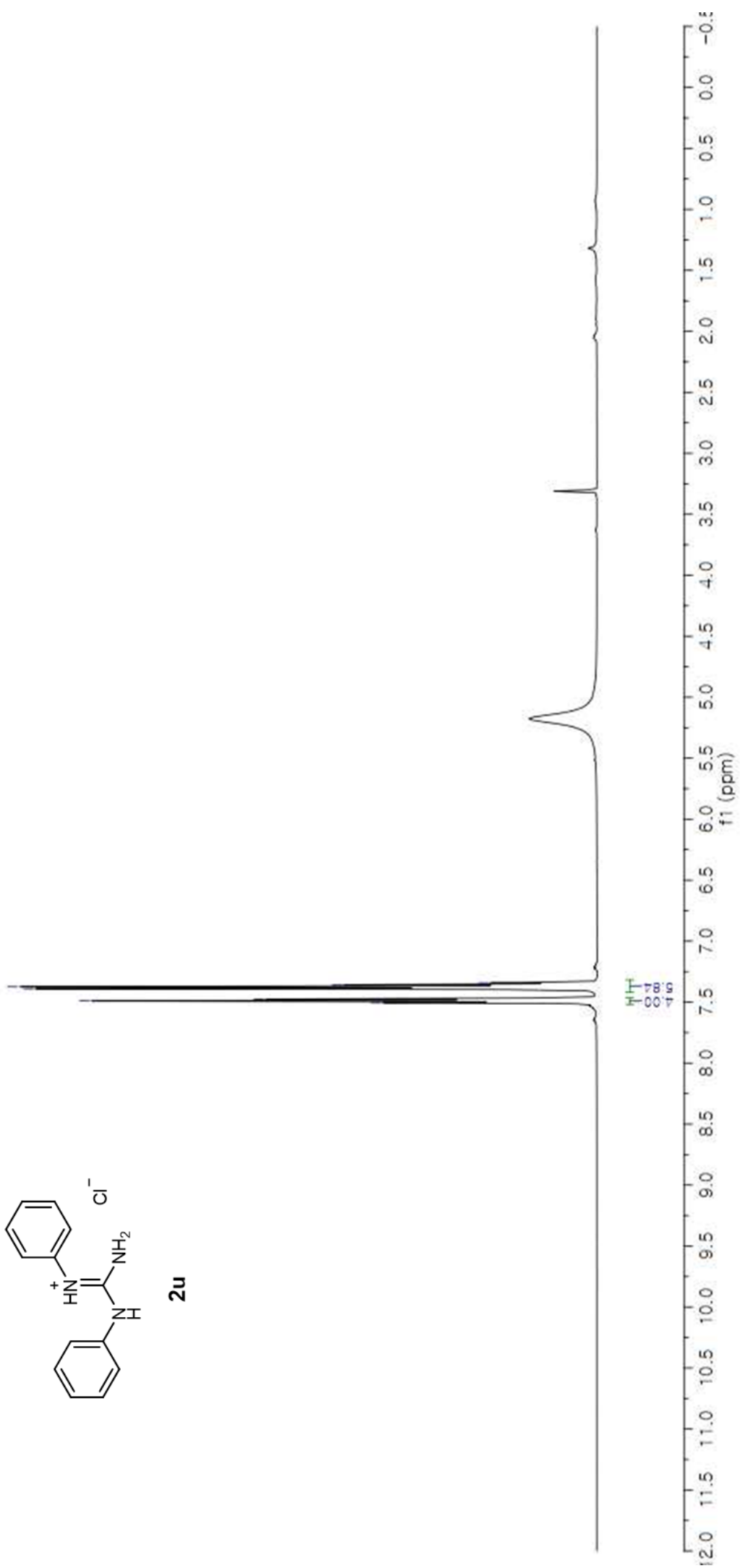




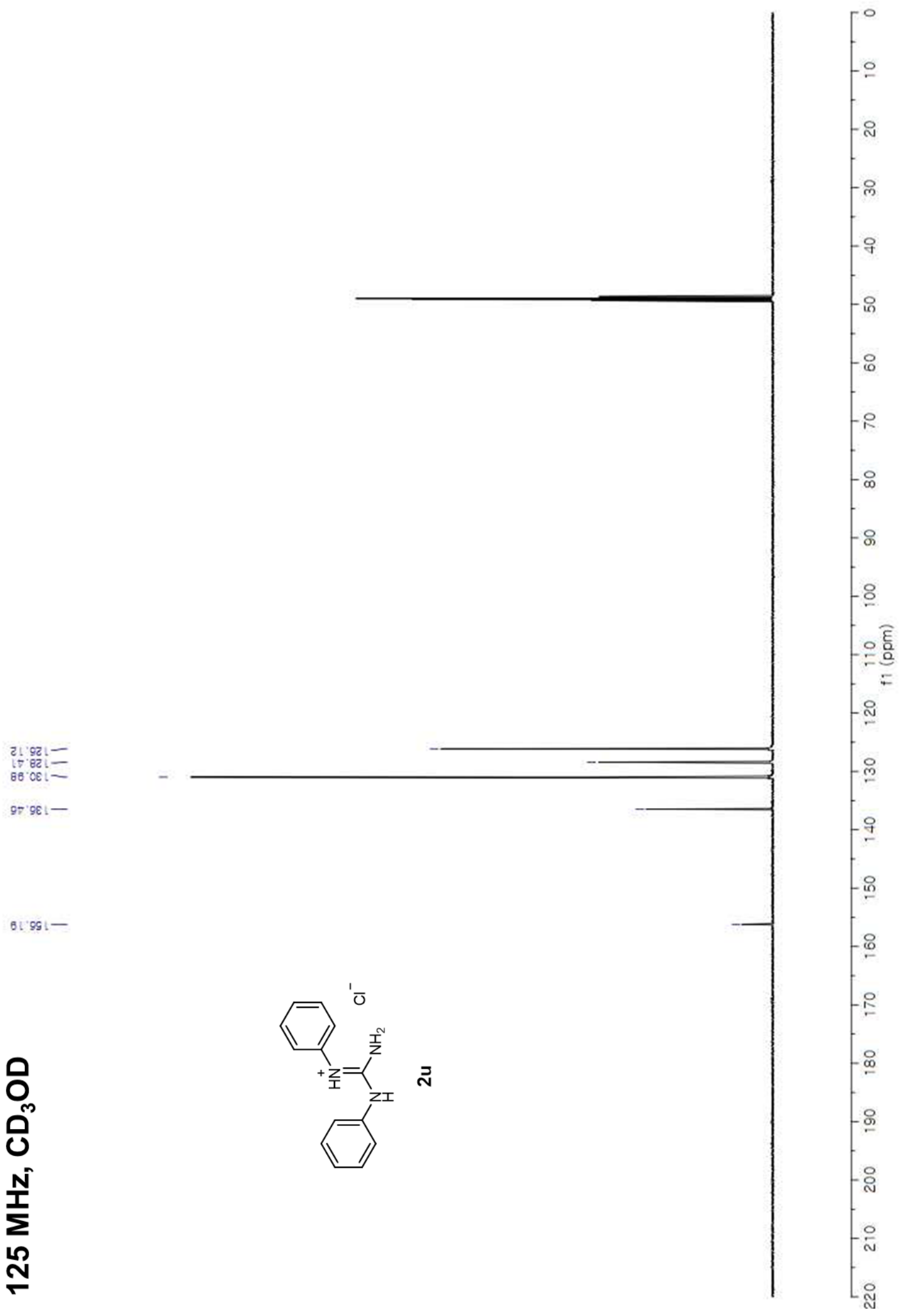




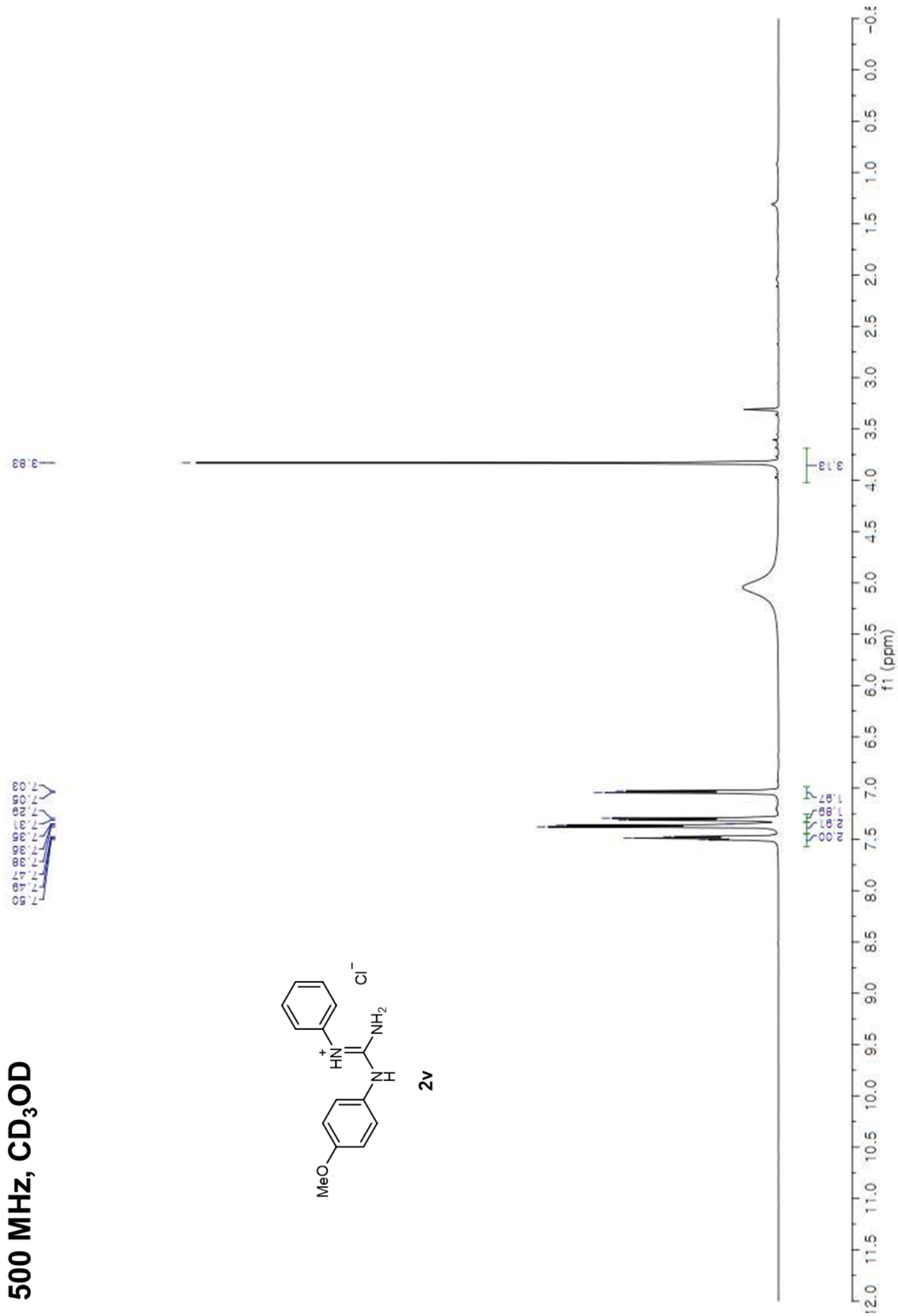




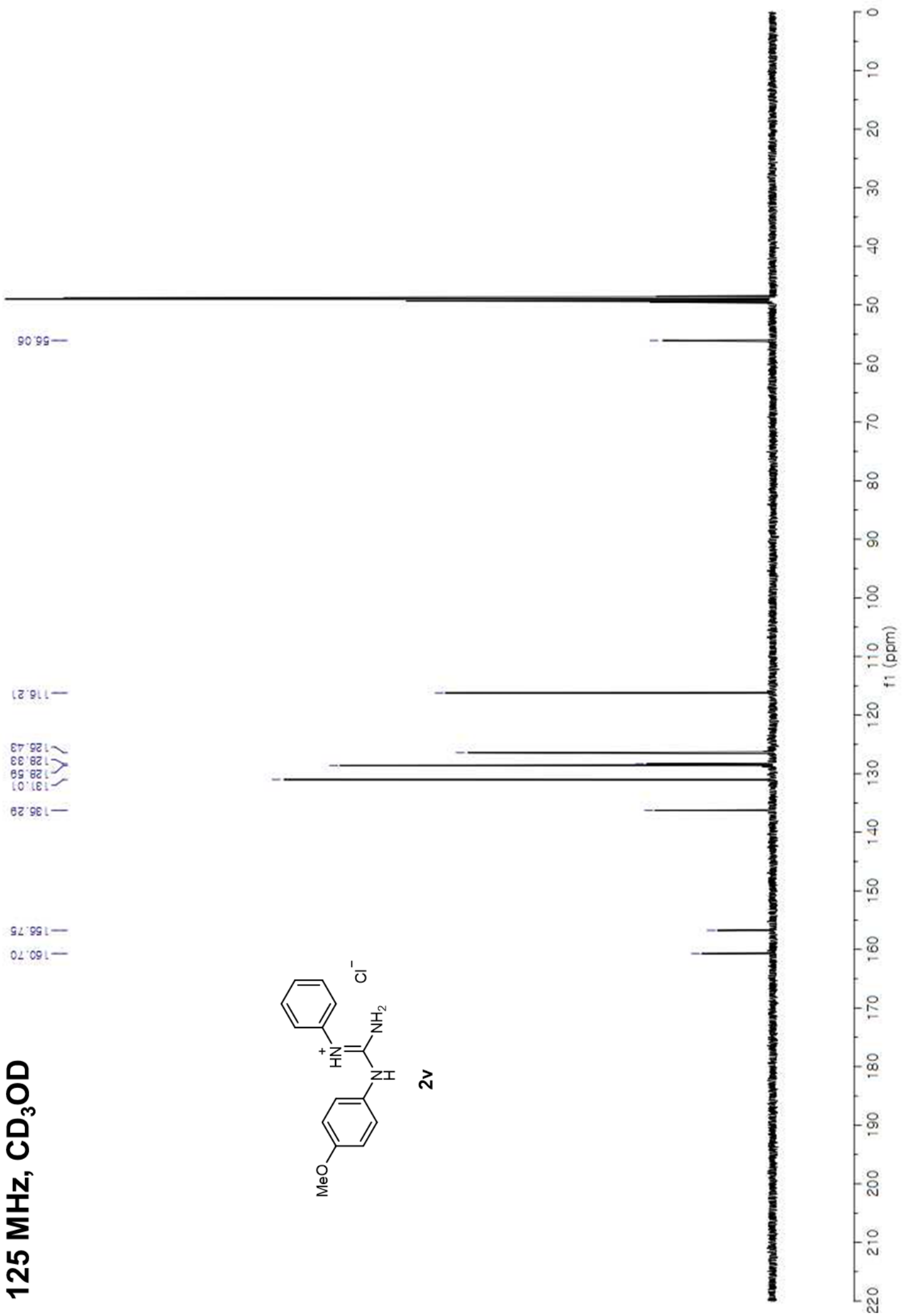




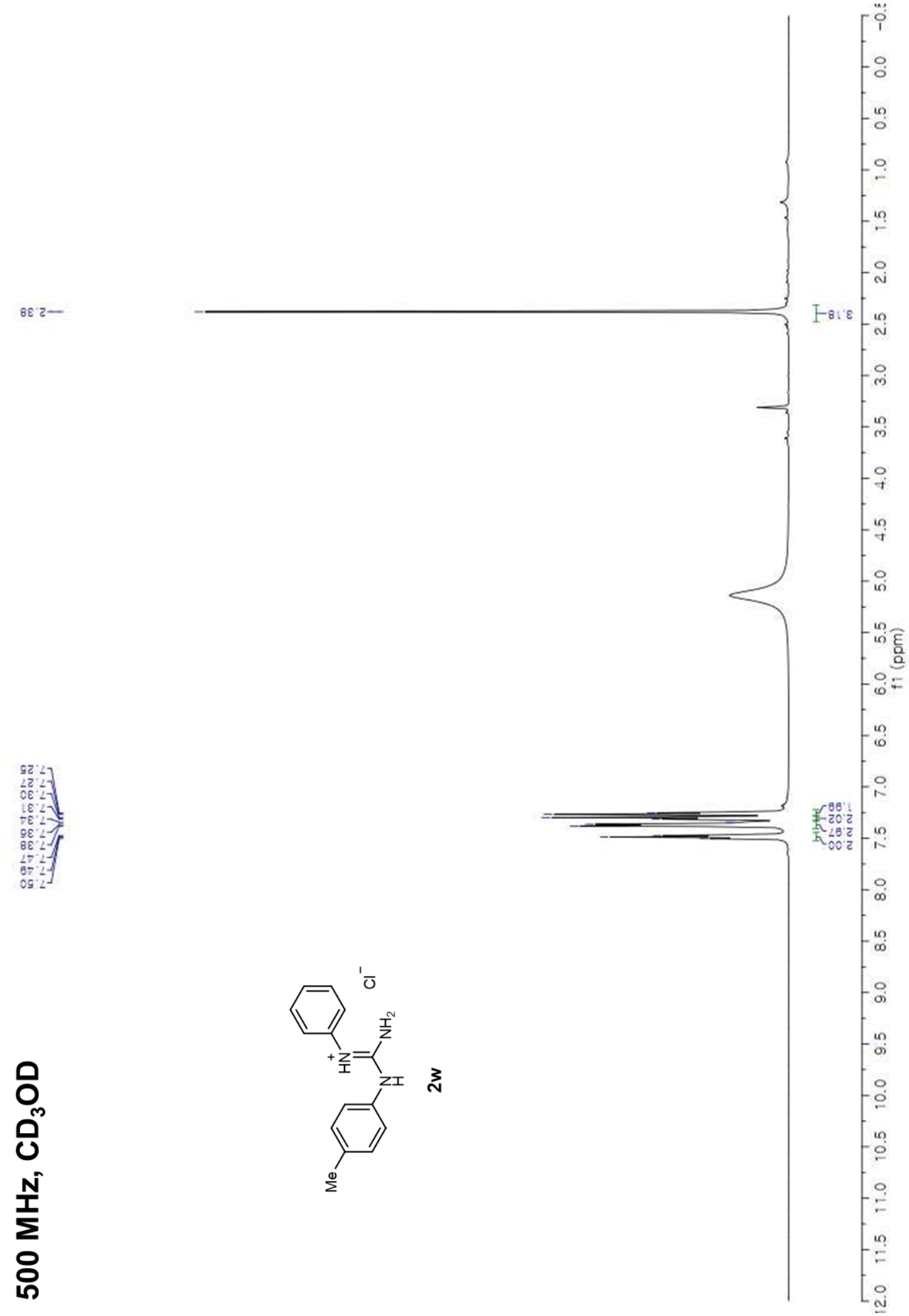




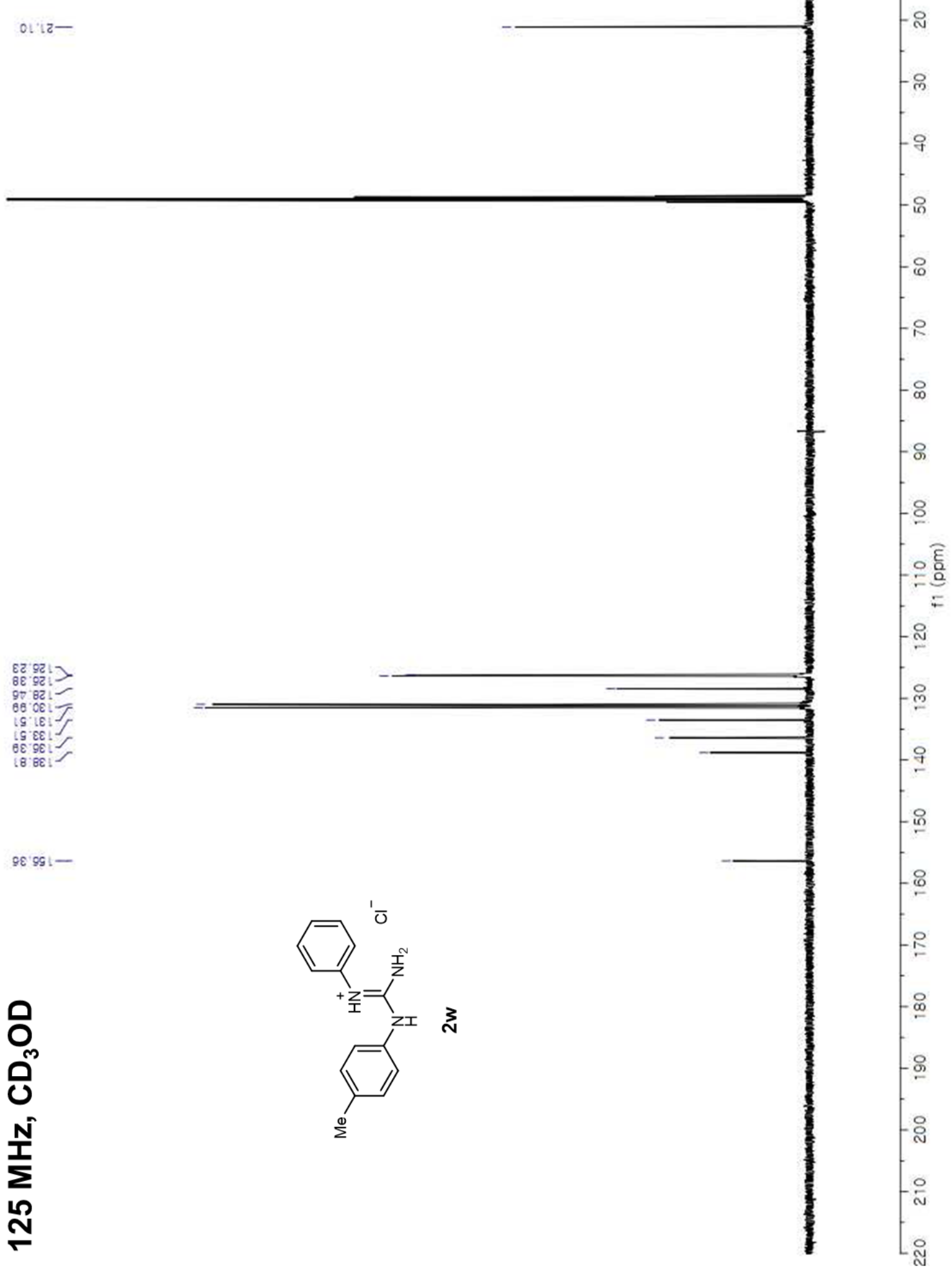




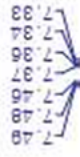

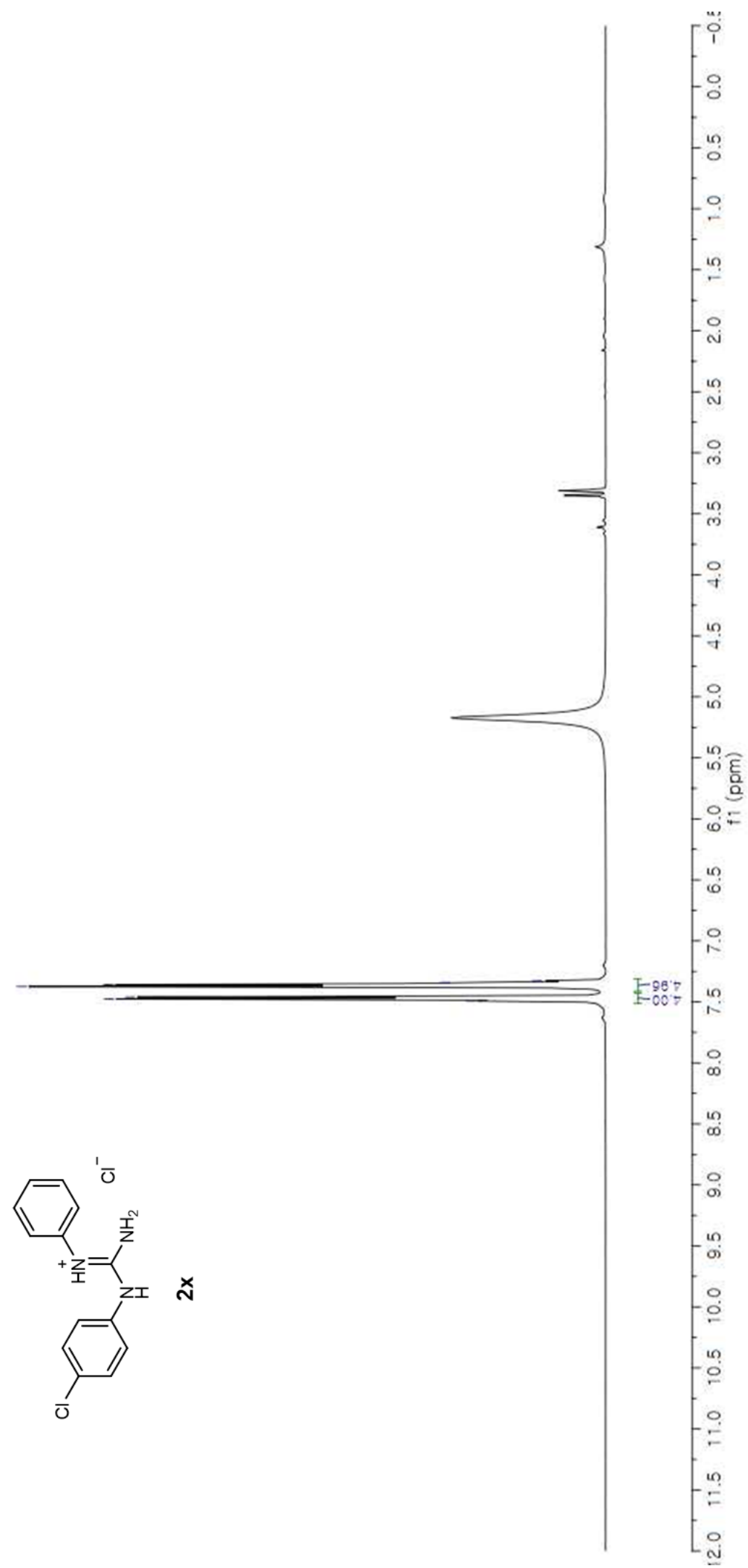




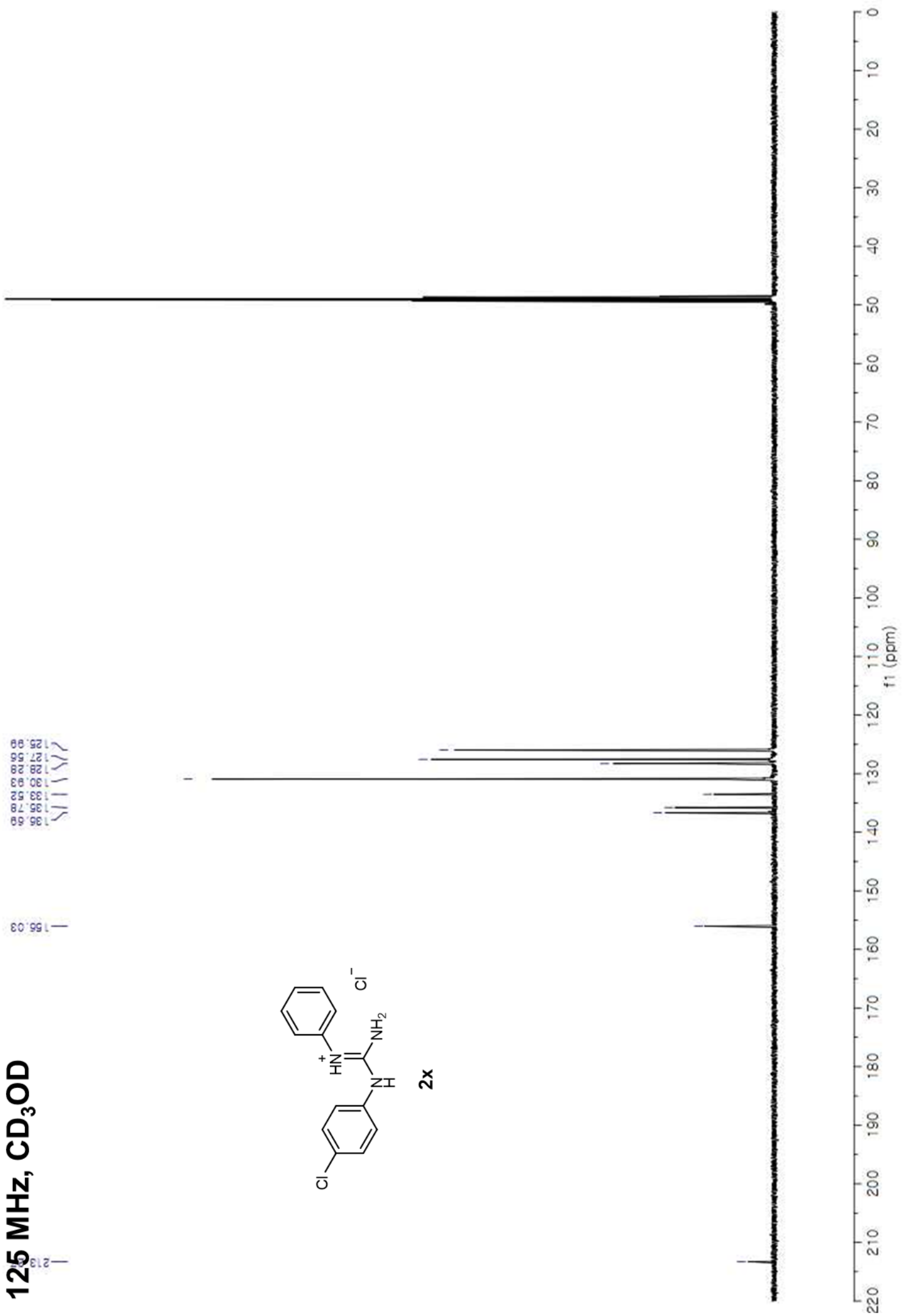




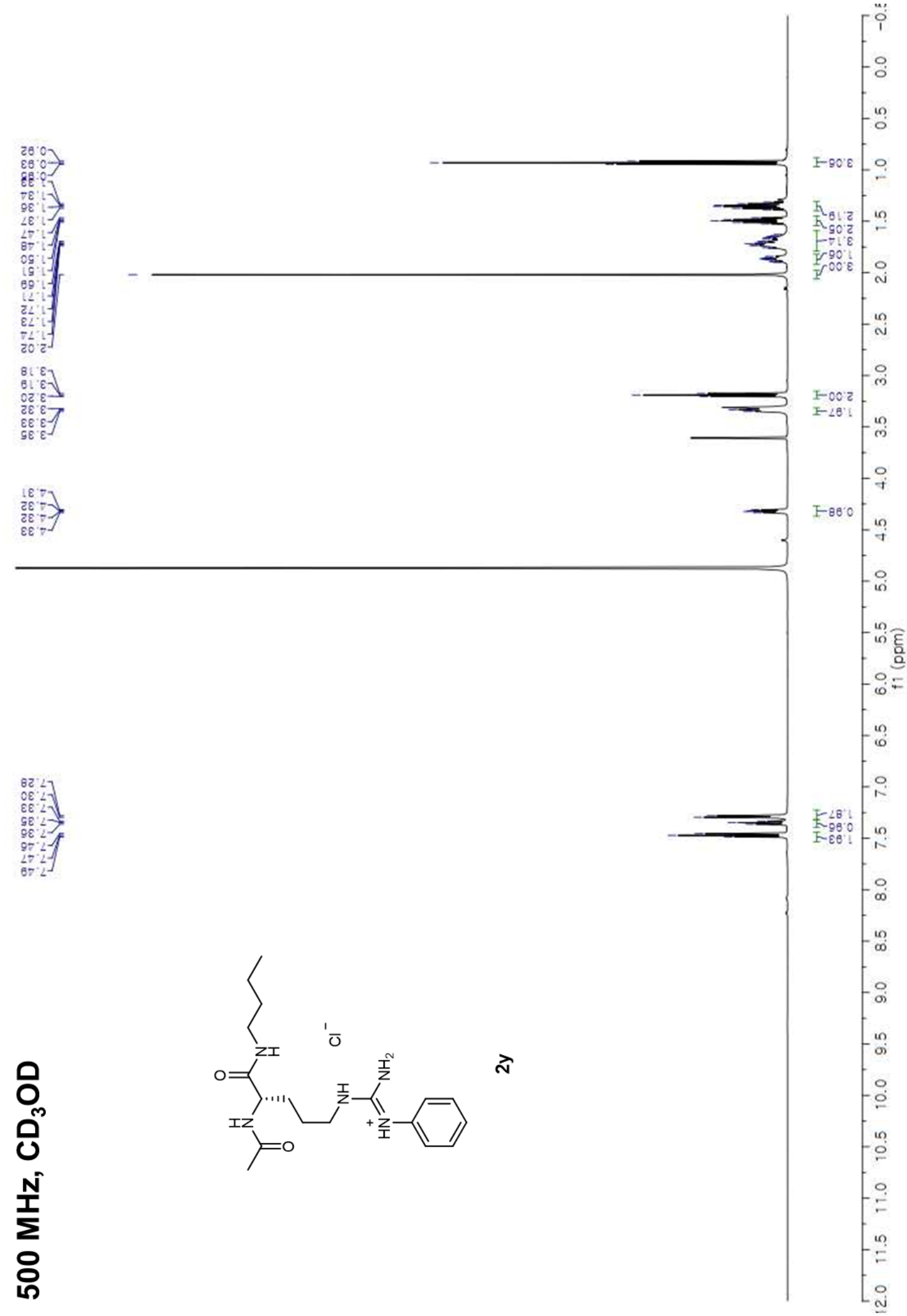




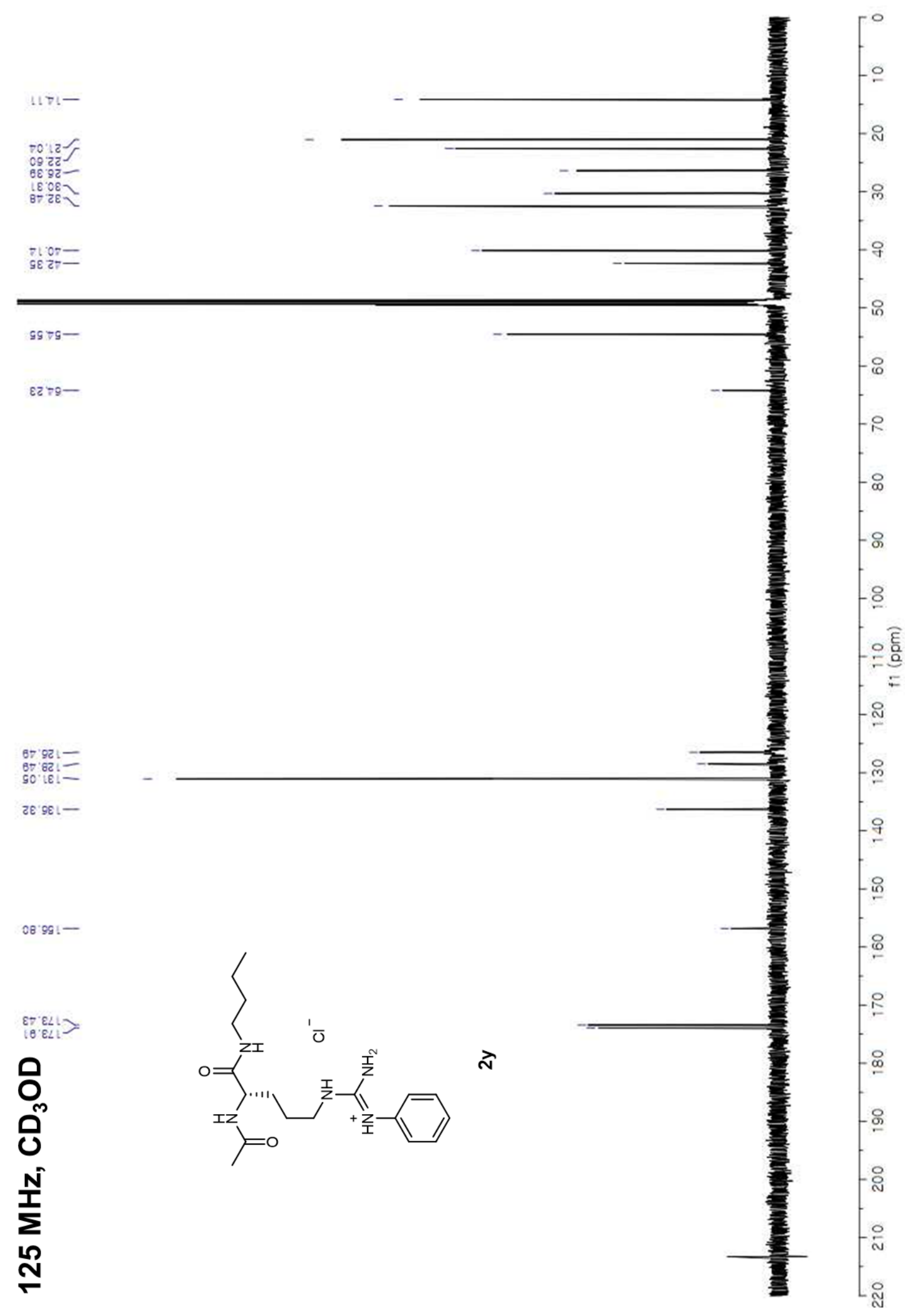




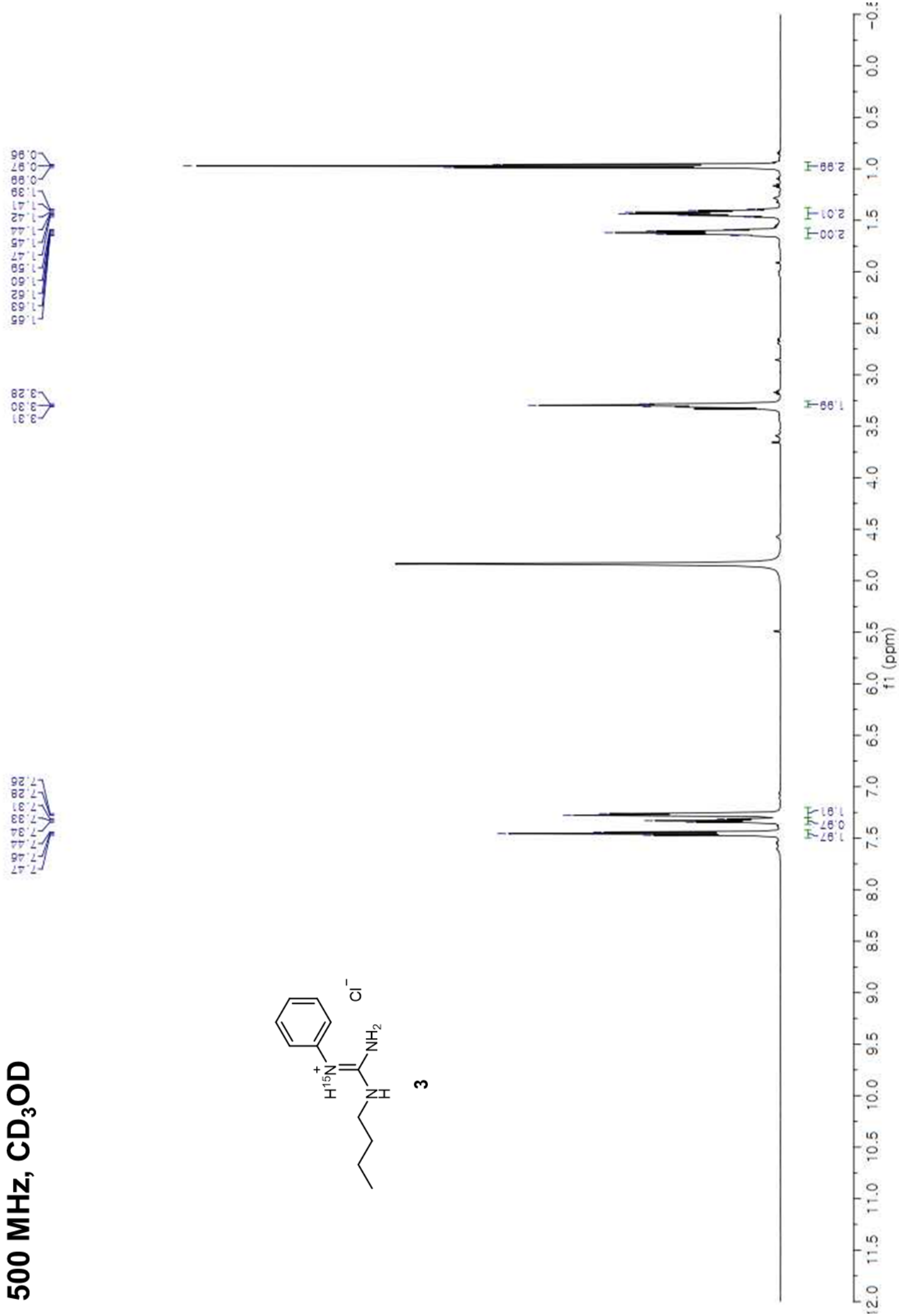




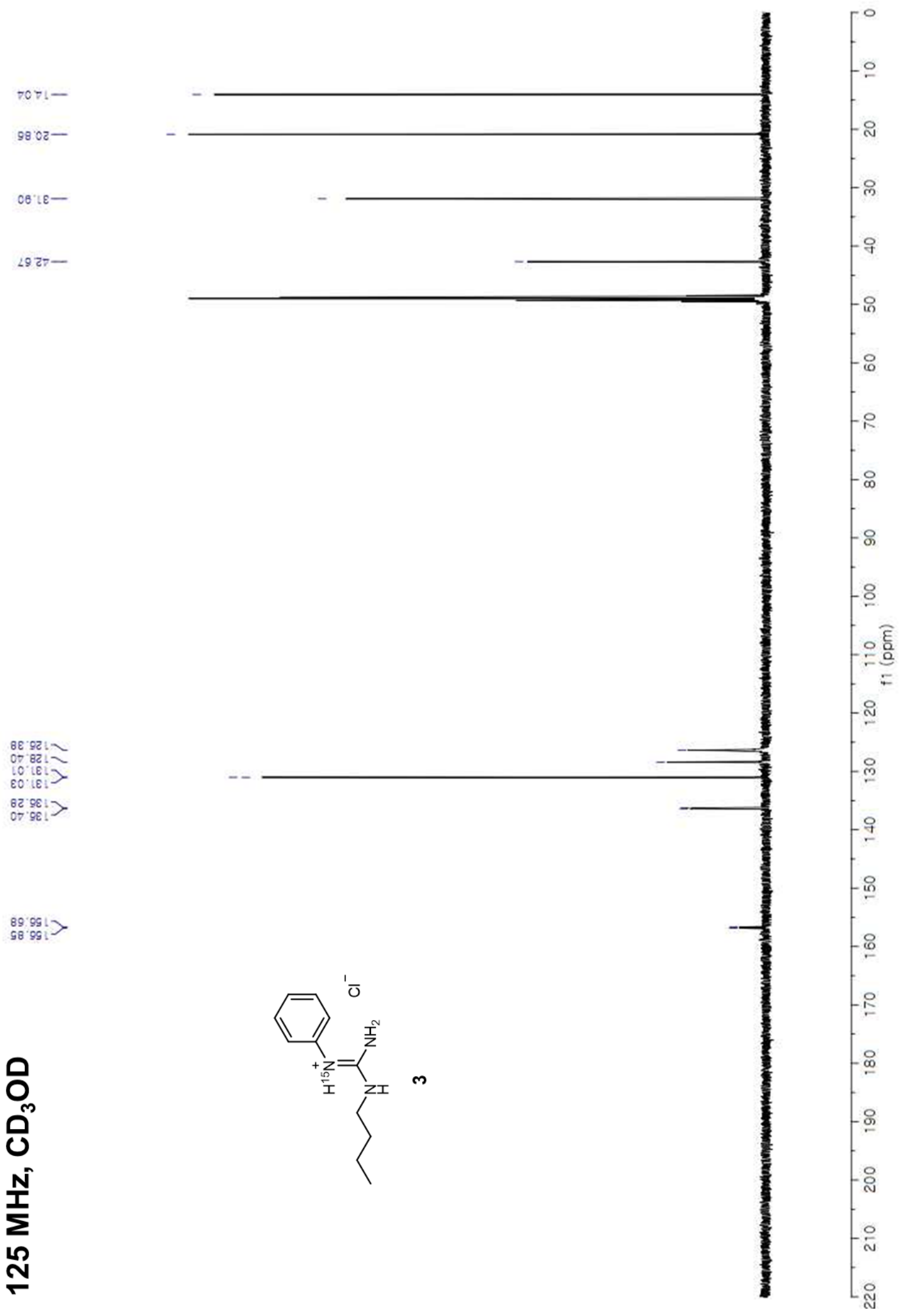



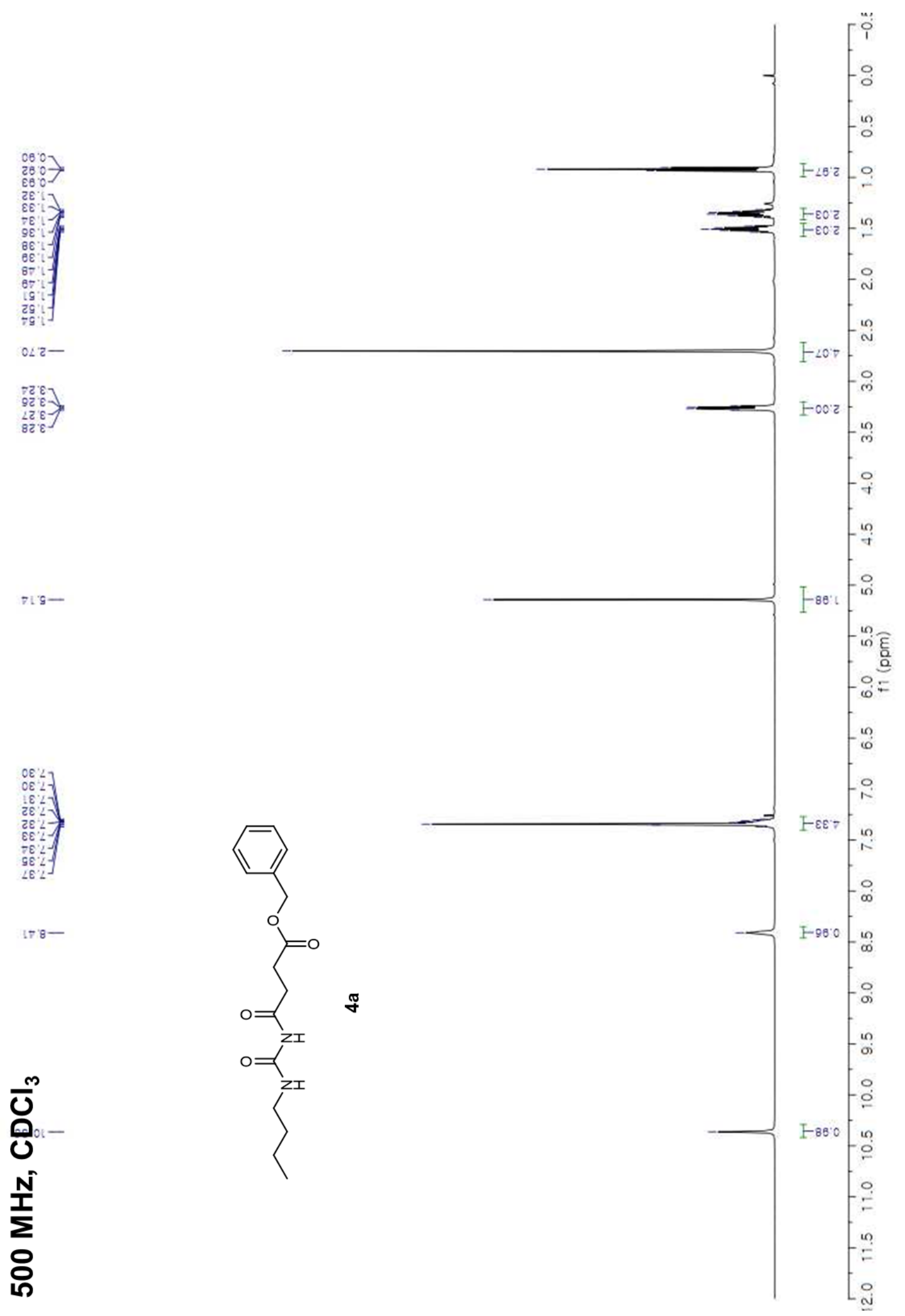


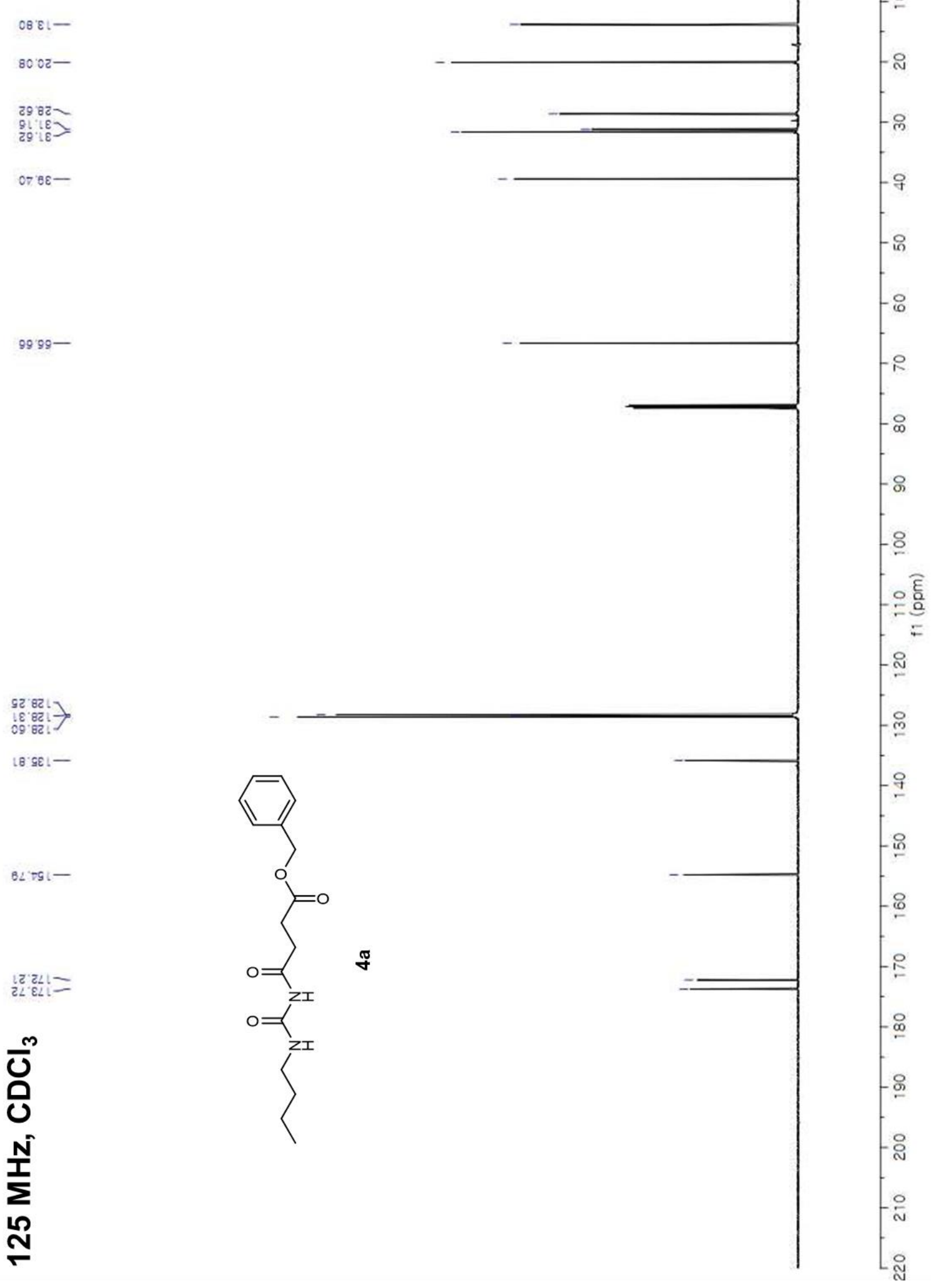




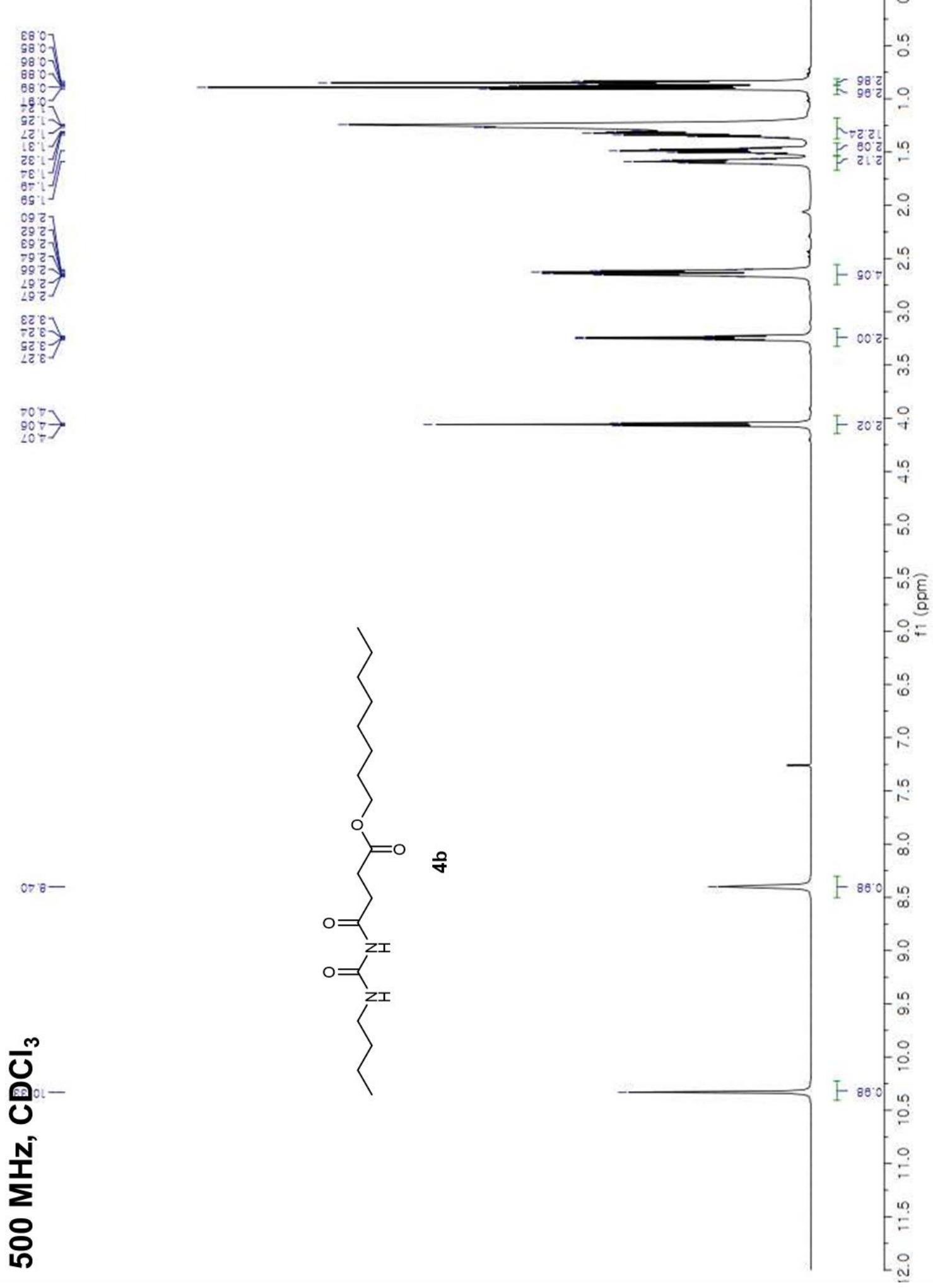



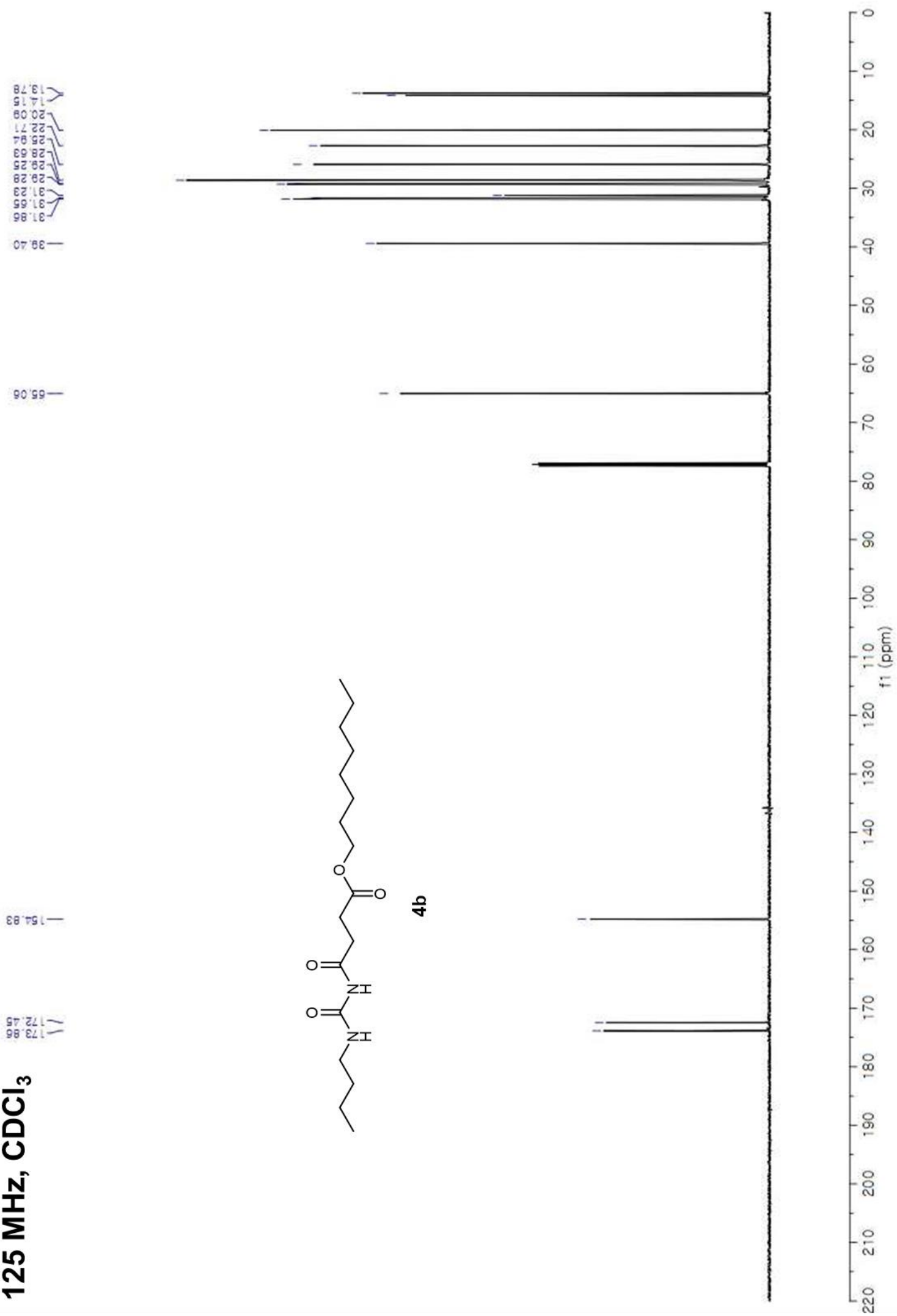

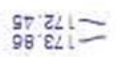

$\overline{0}$
Oे
$\stackrel{N}{1}$
$\stackrel{N}{N}$ 


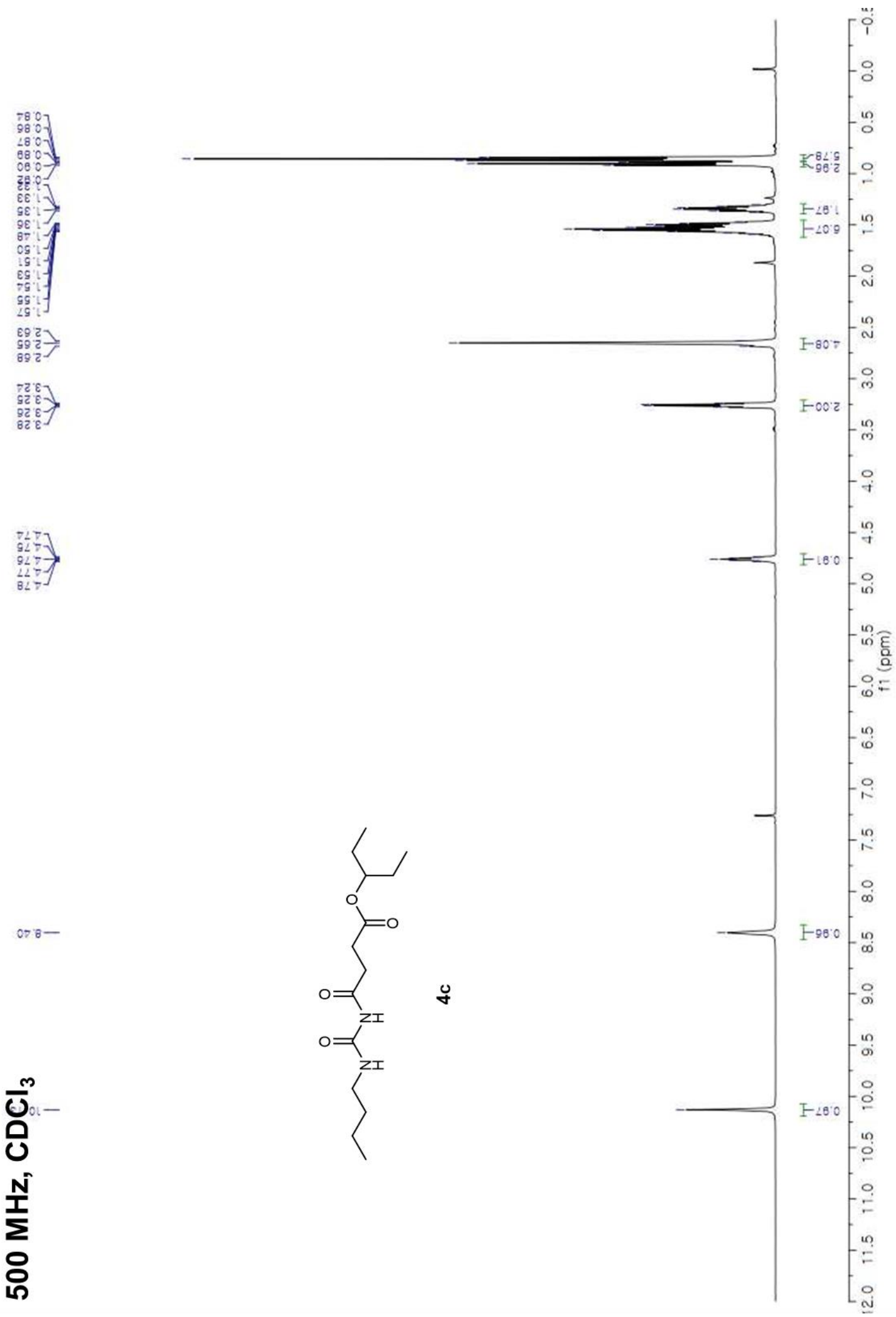



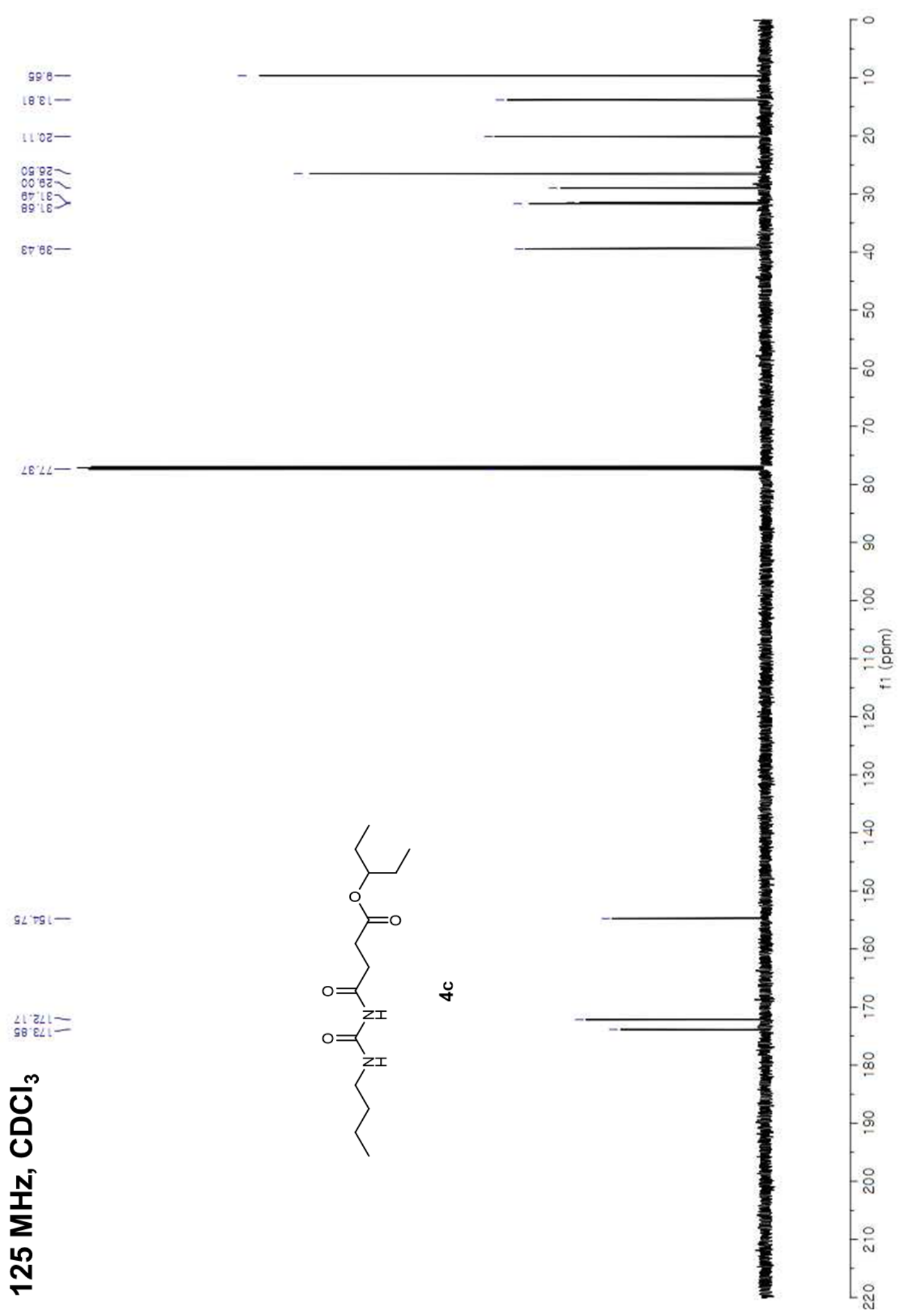


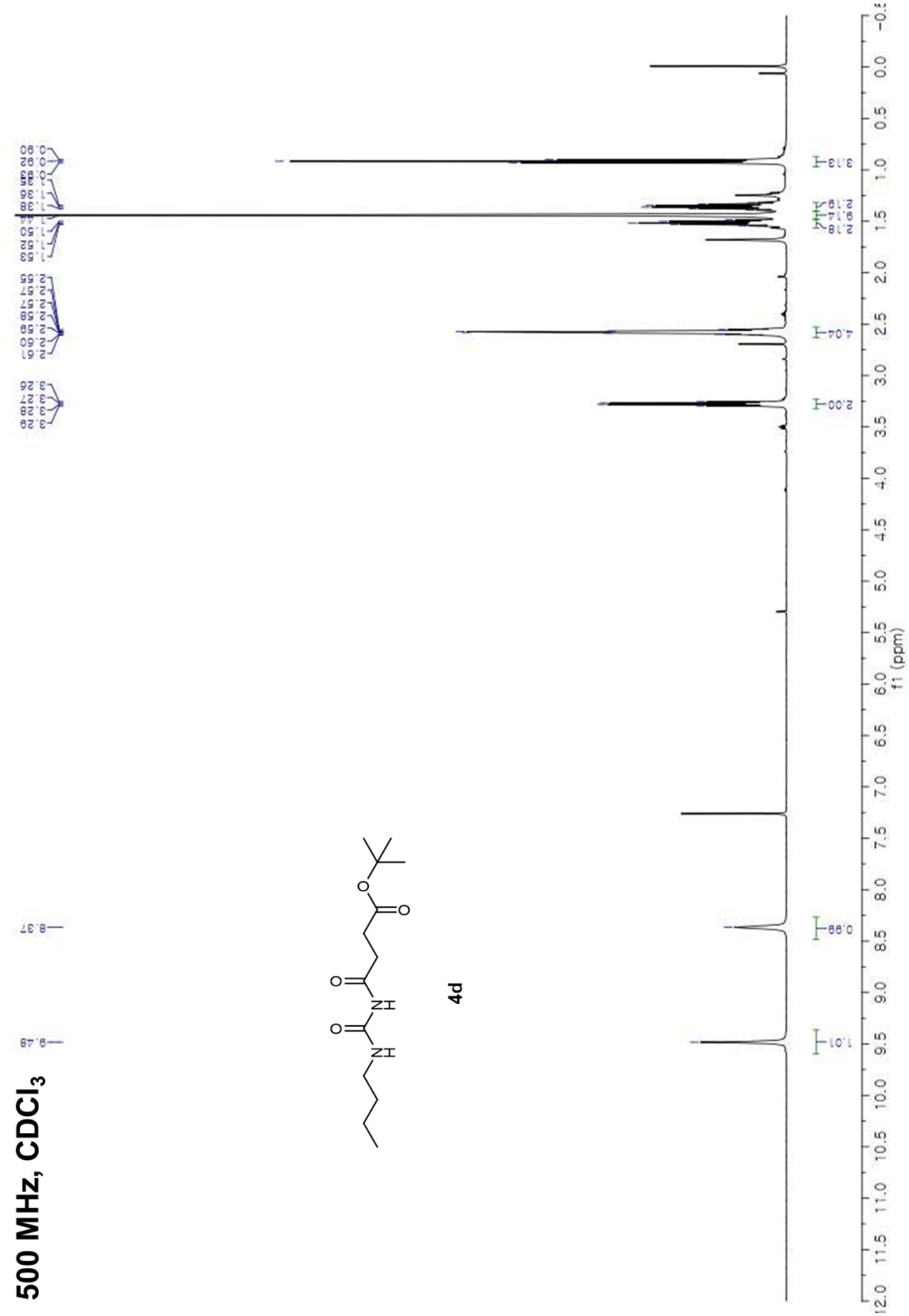




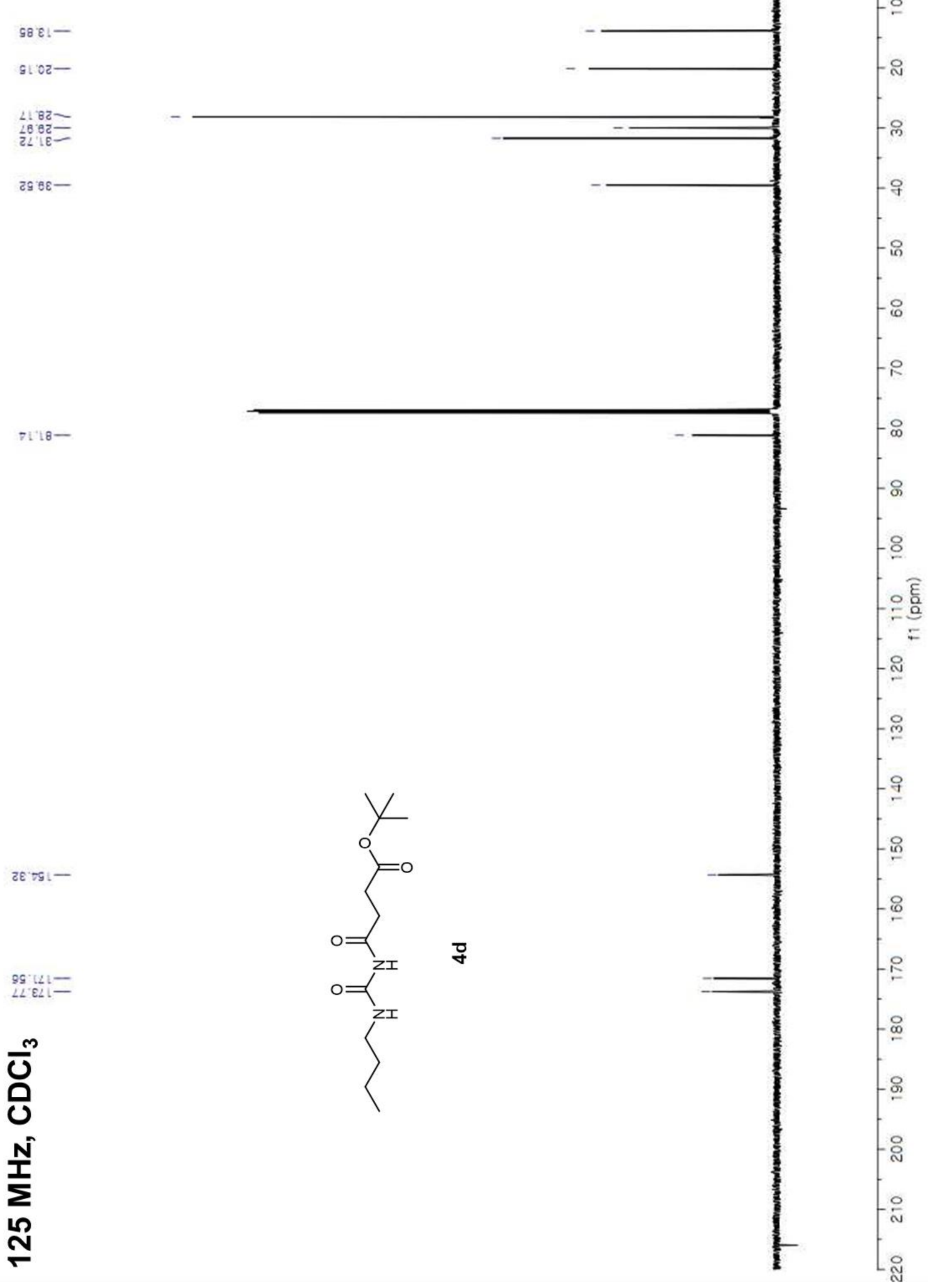




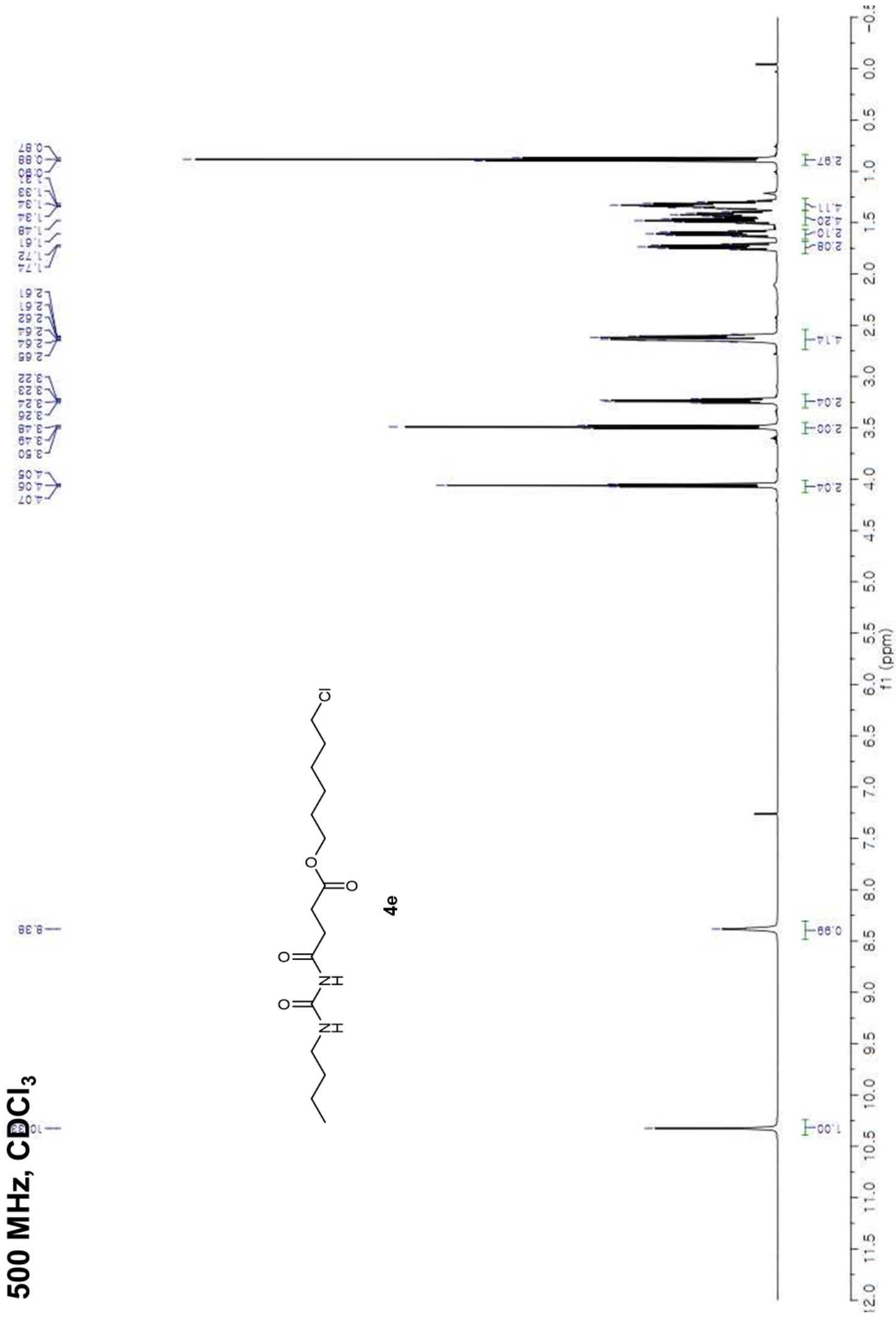




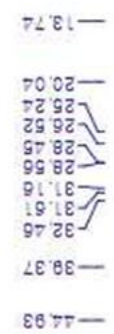

$0279-$

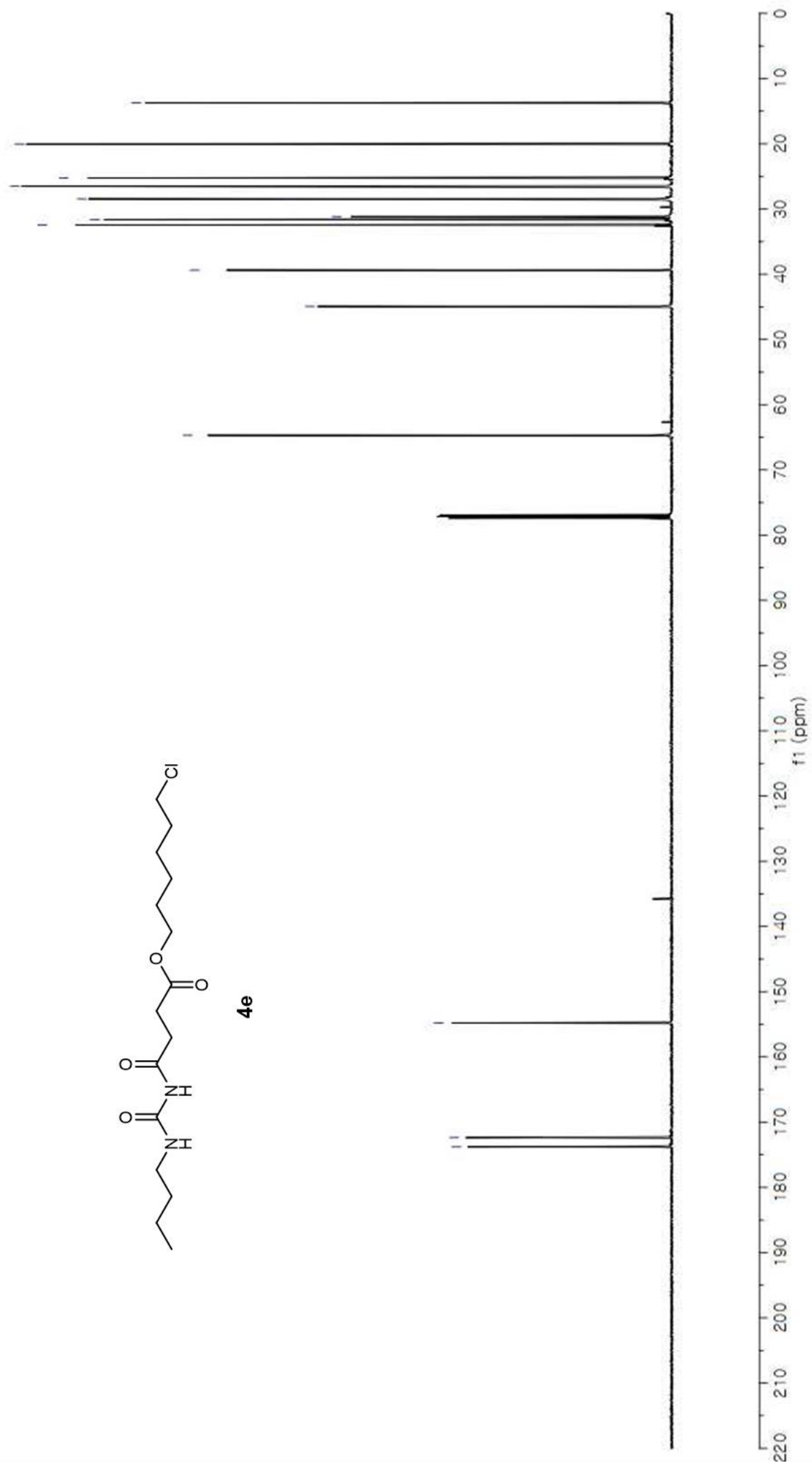

${ }_{08 \varepsilon L}^{8 \varepsilon} z L=$

BL 79 L-

$\frac{m}{0}$
0
N
I
N 


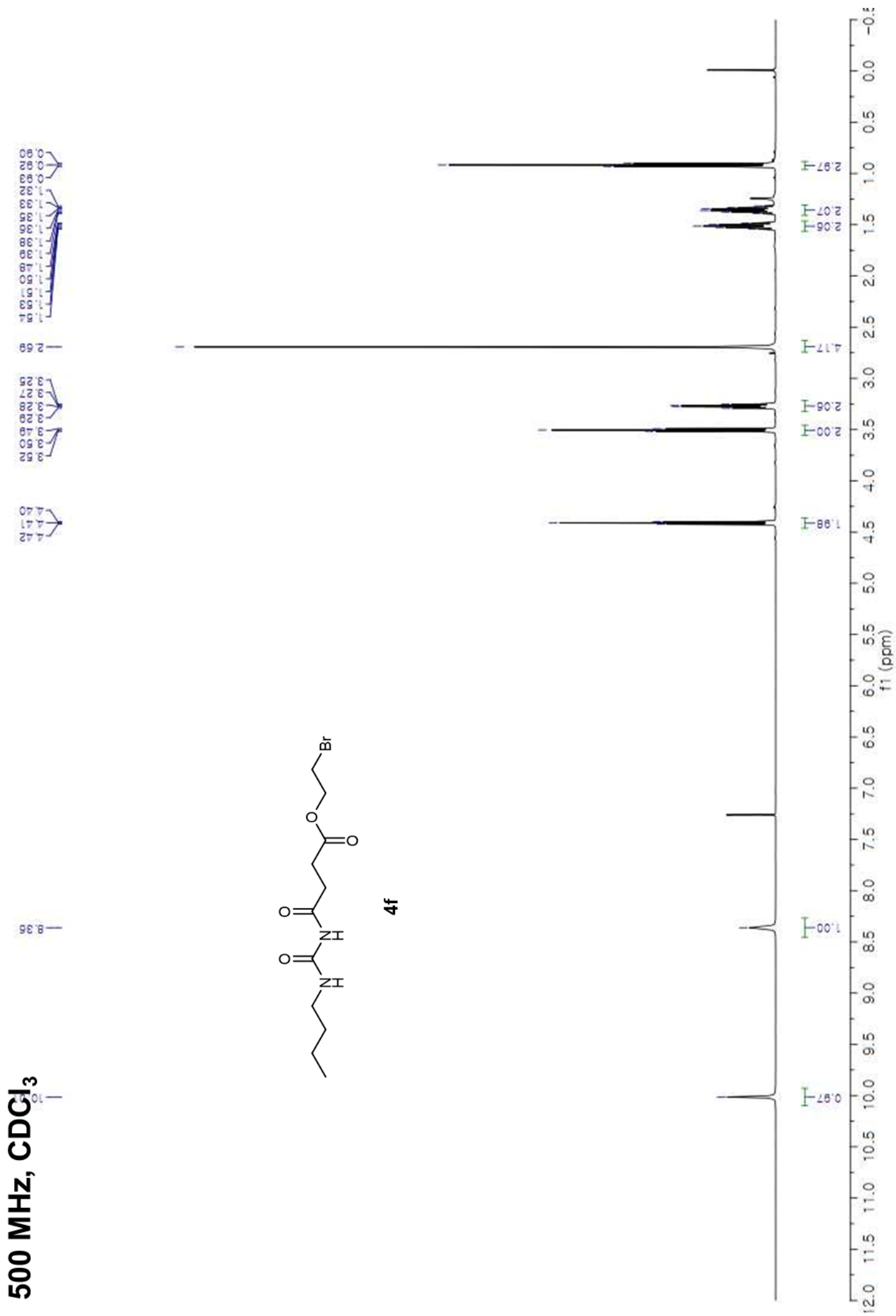




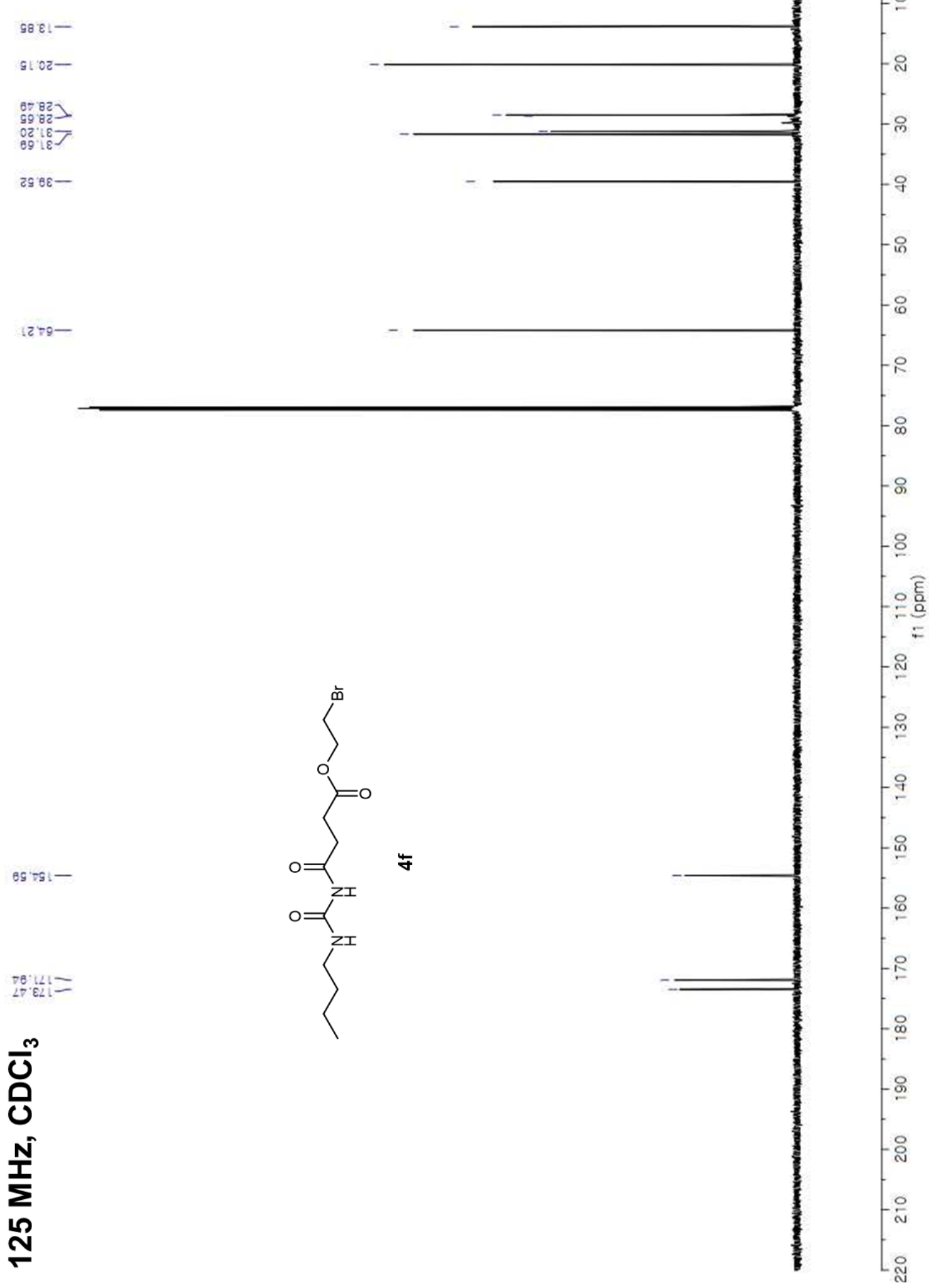



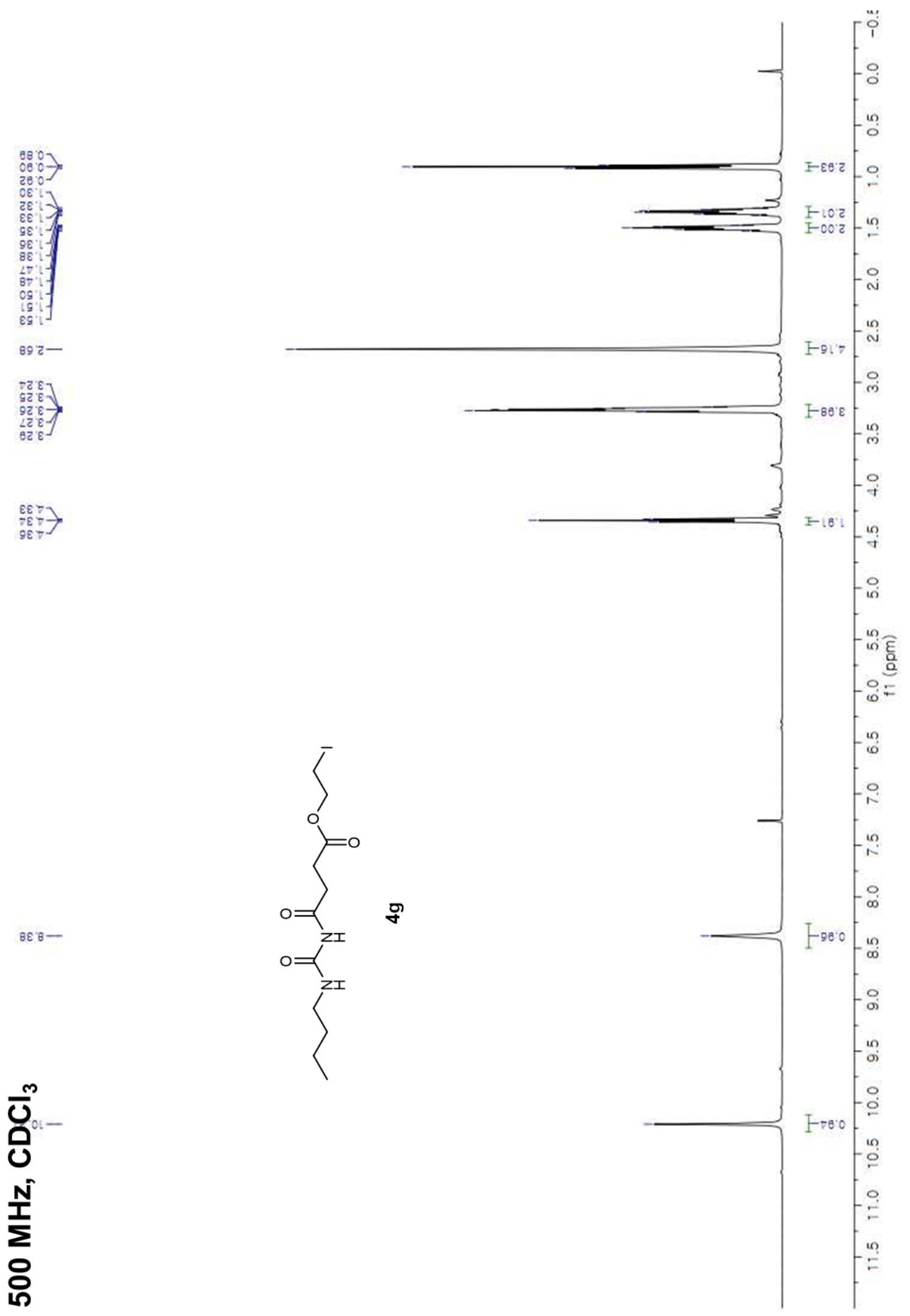


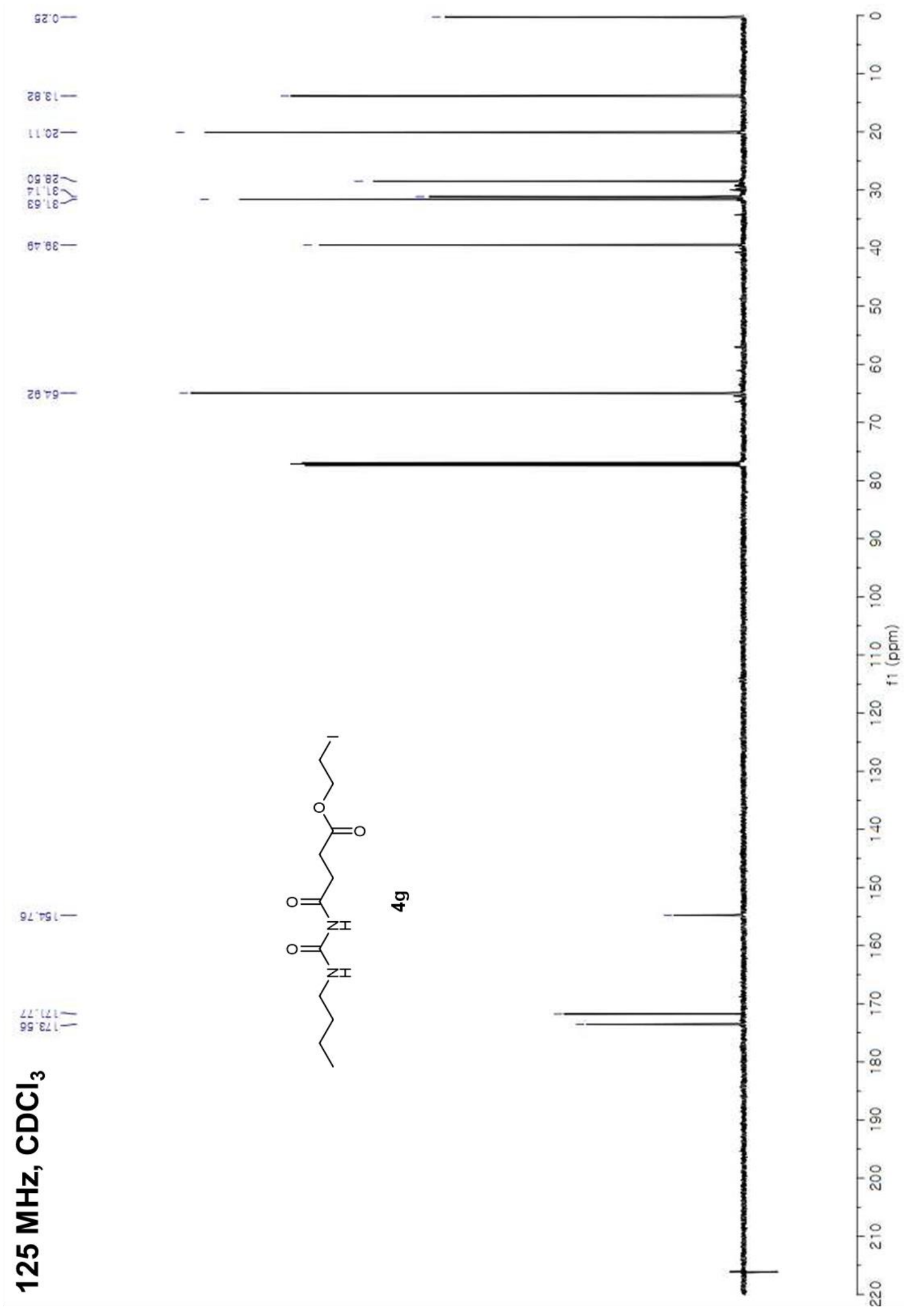




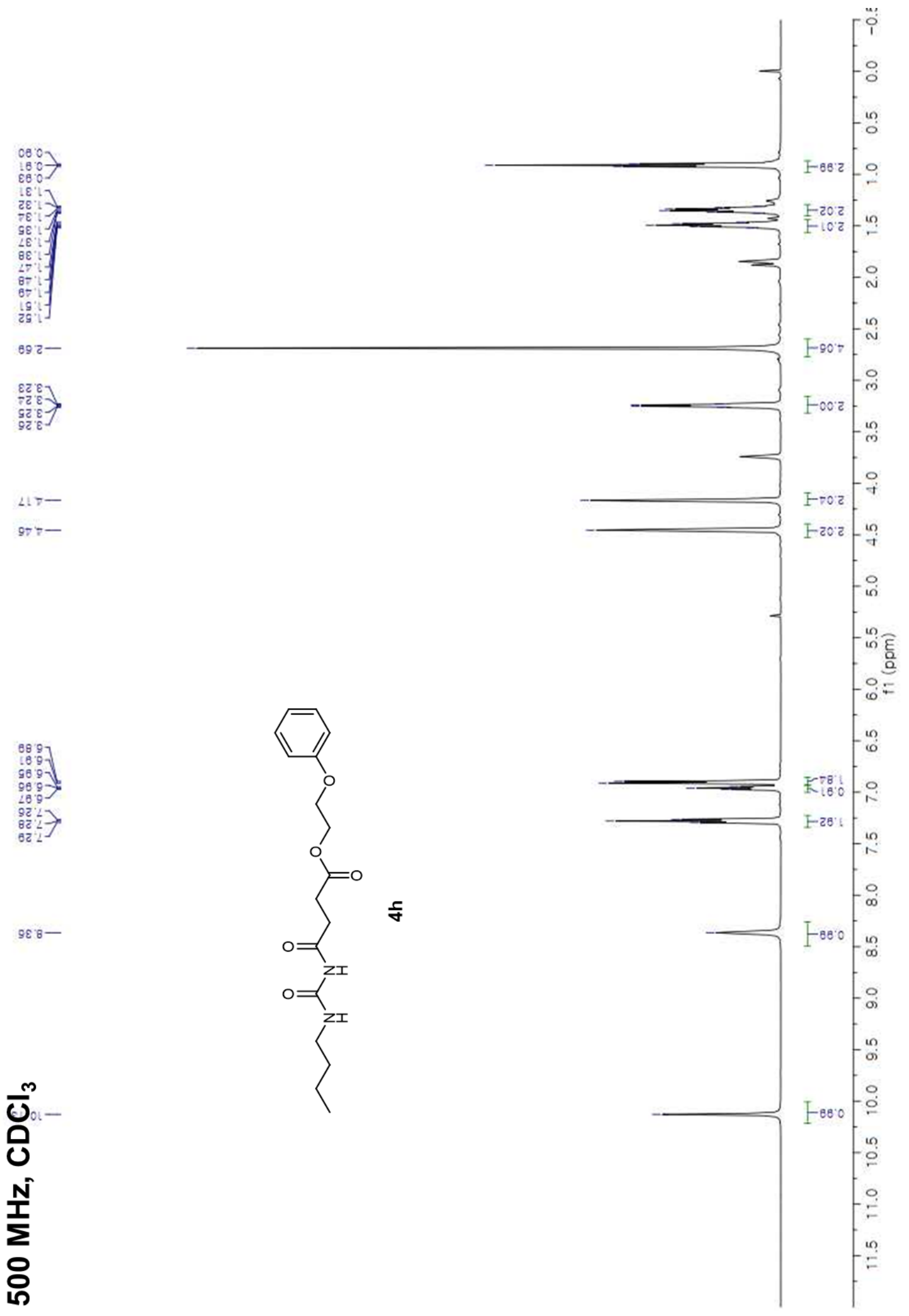



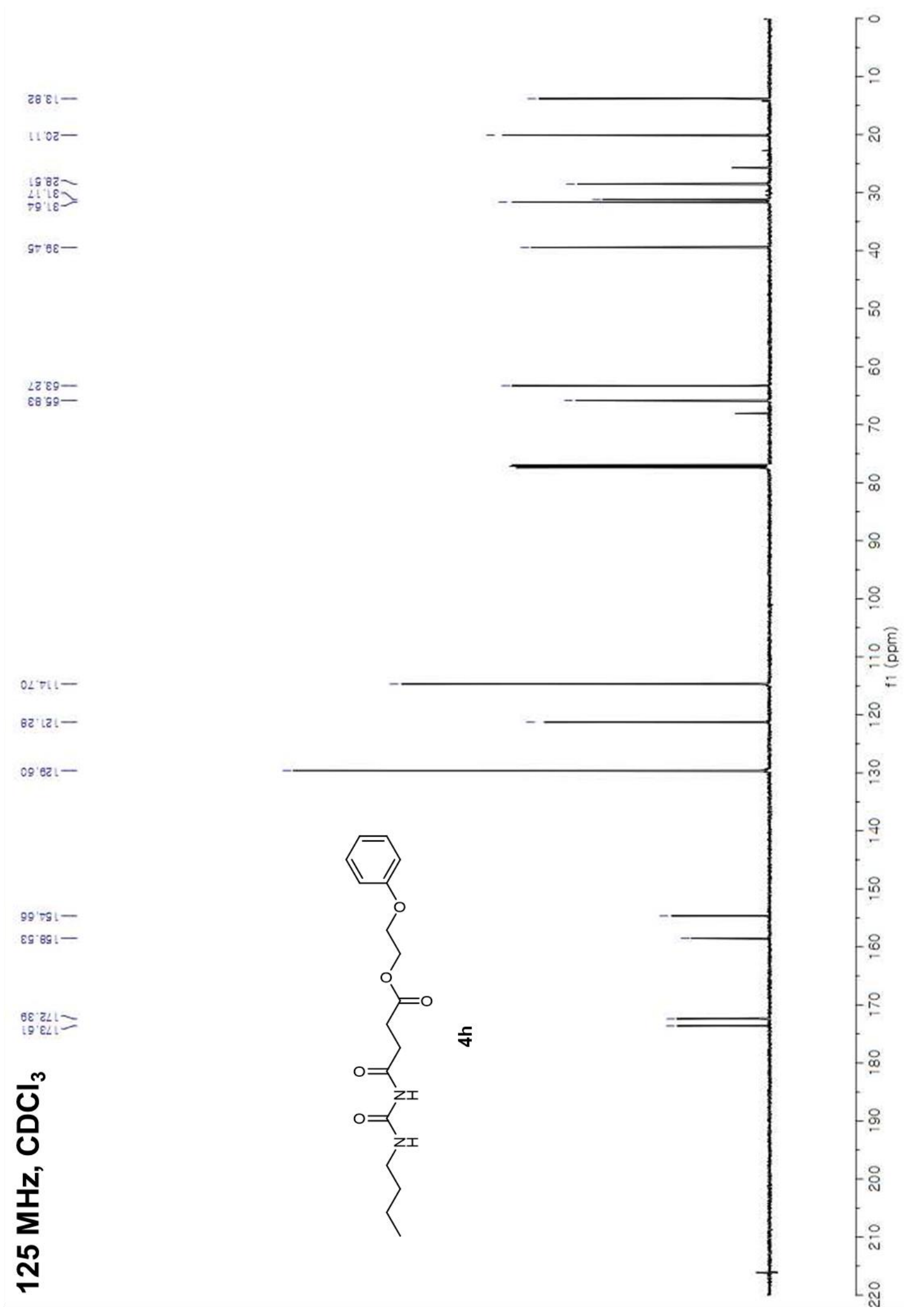


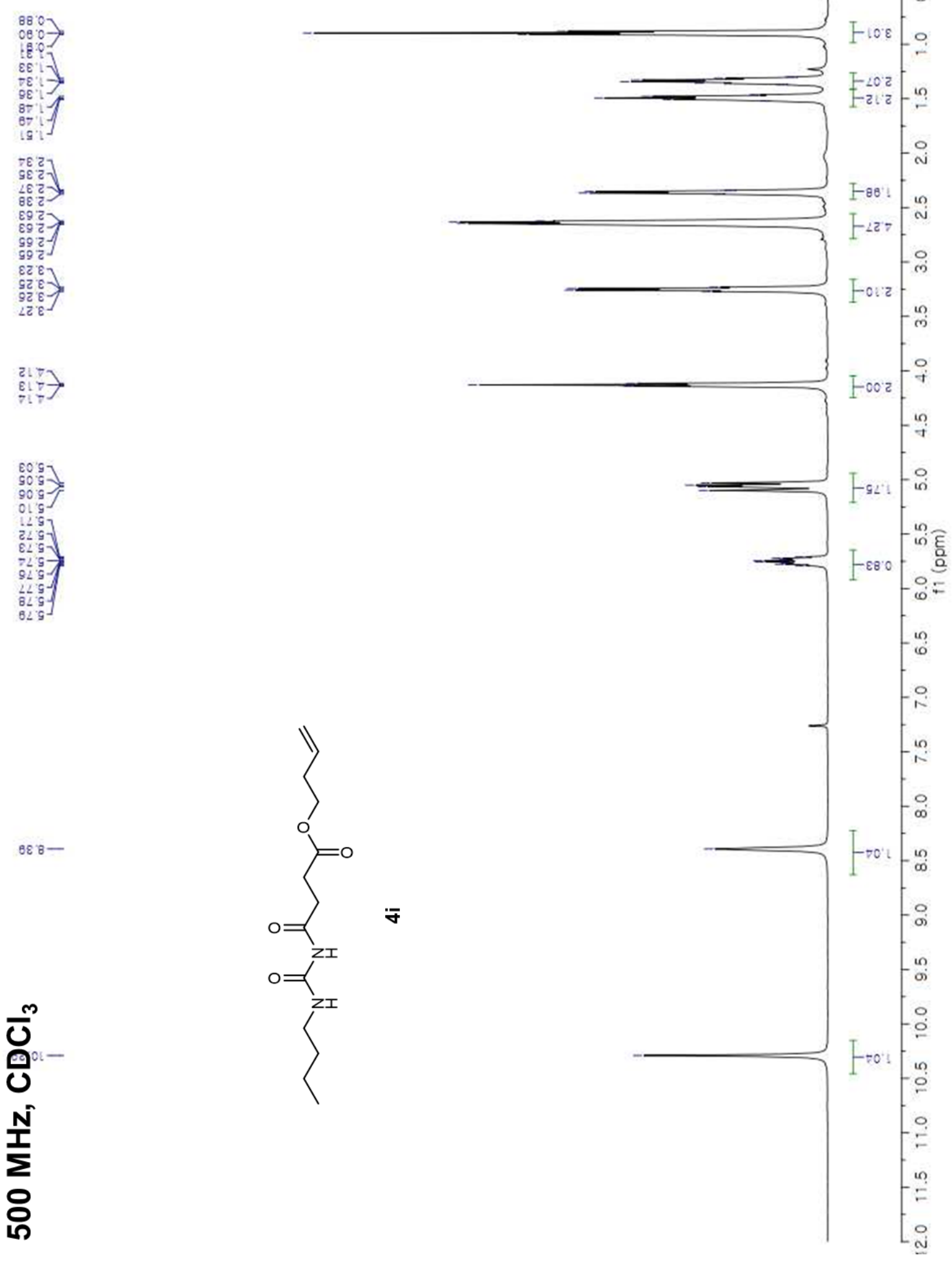




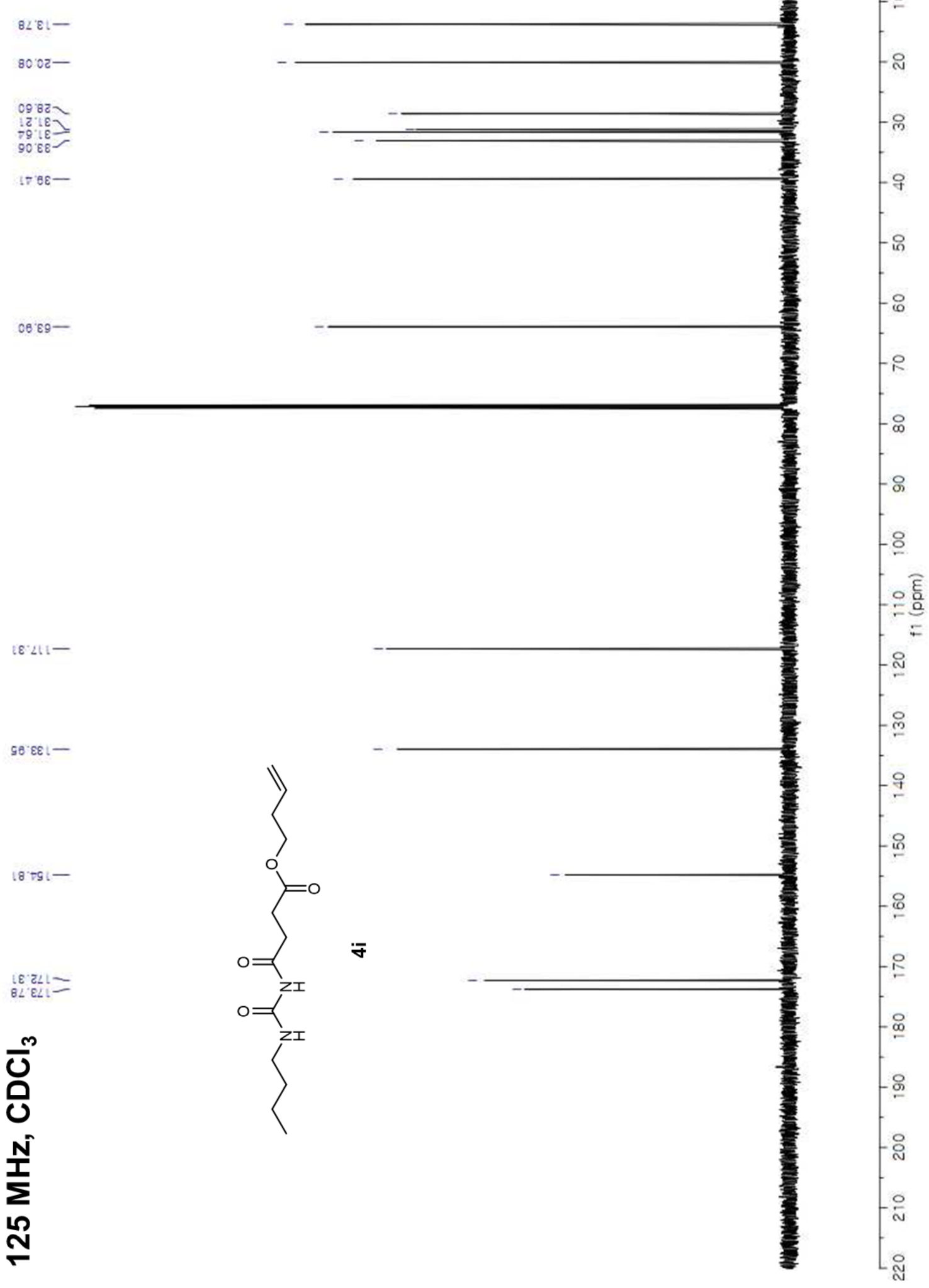



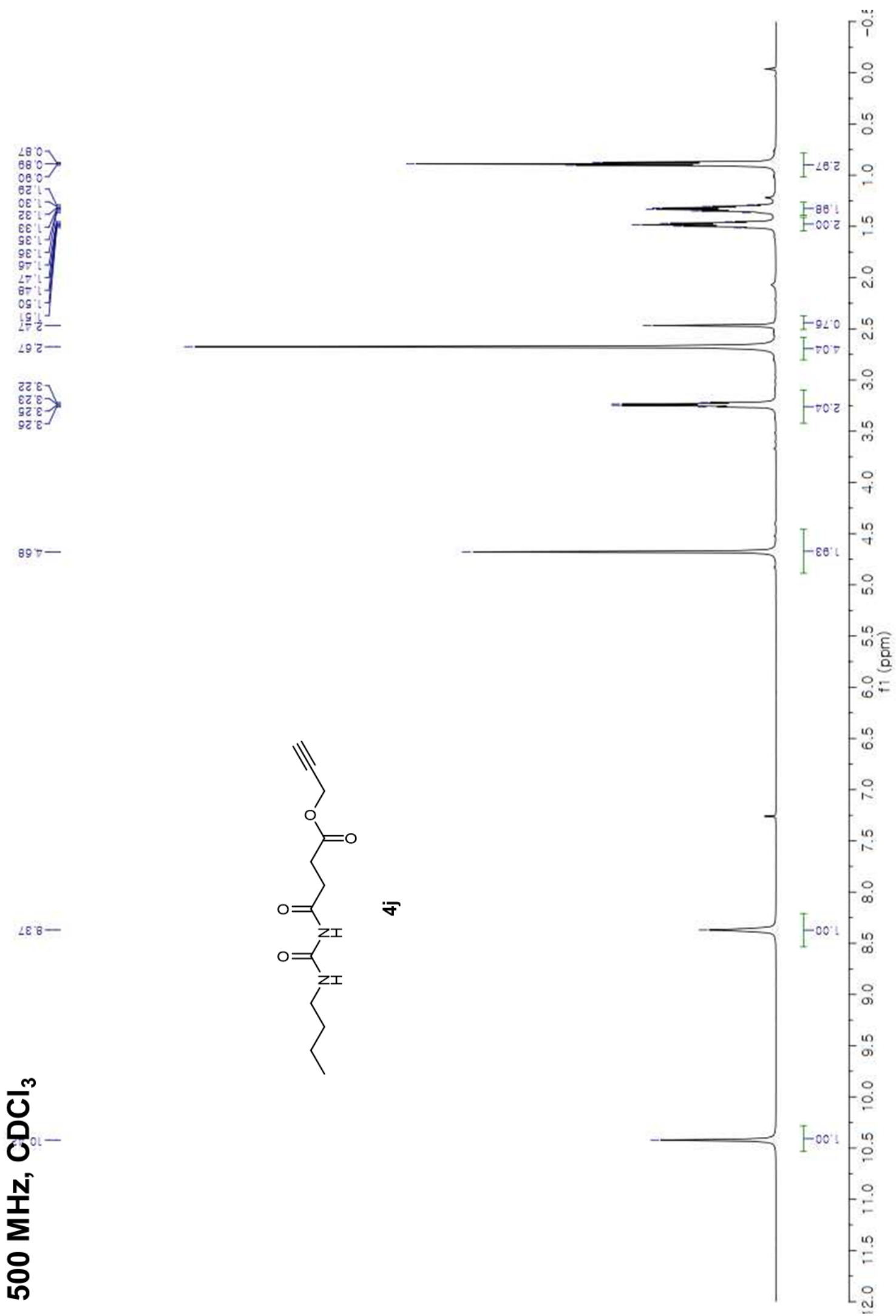


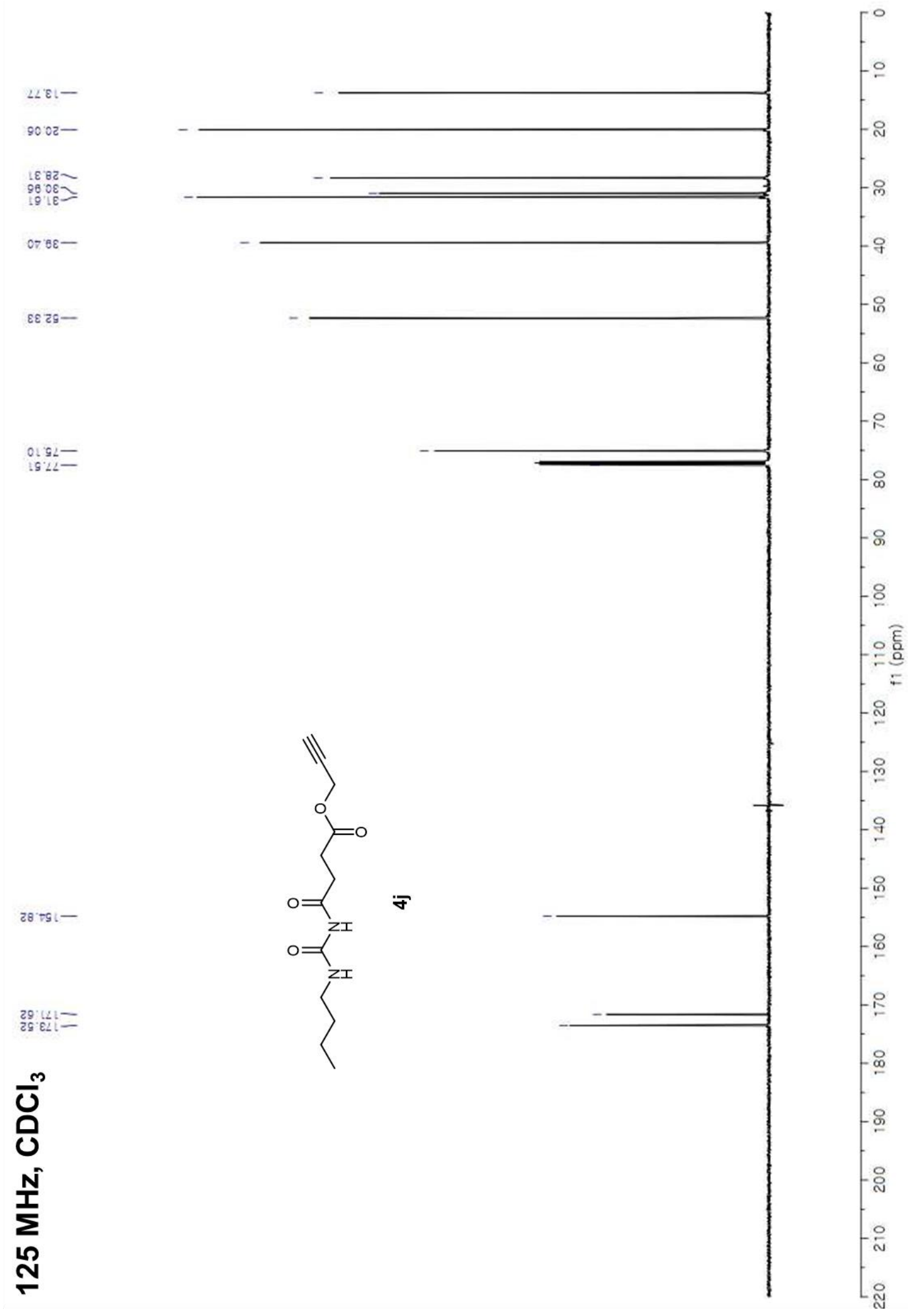



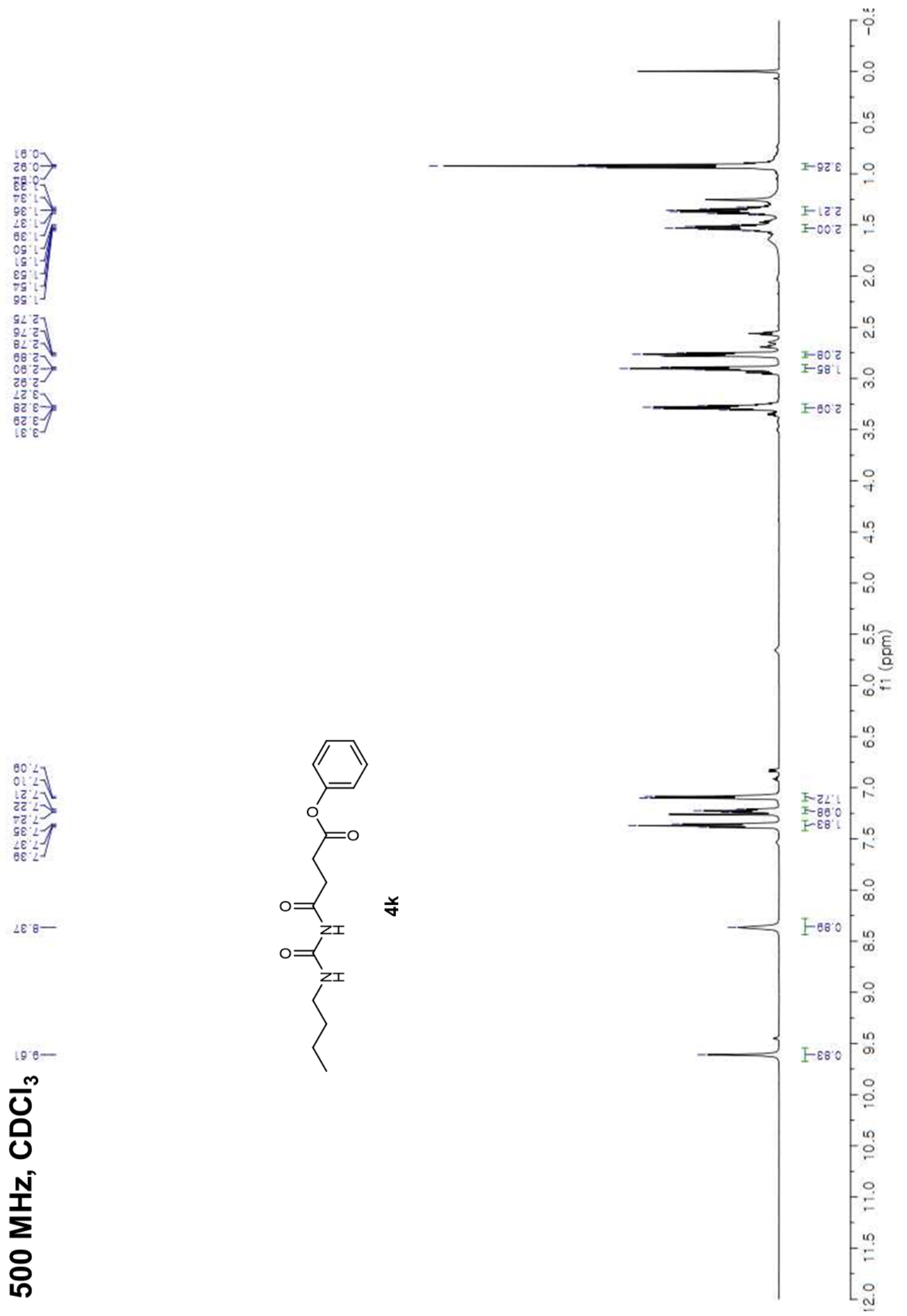


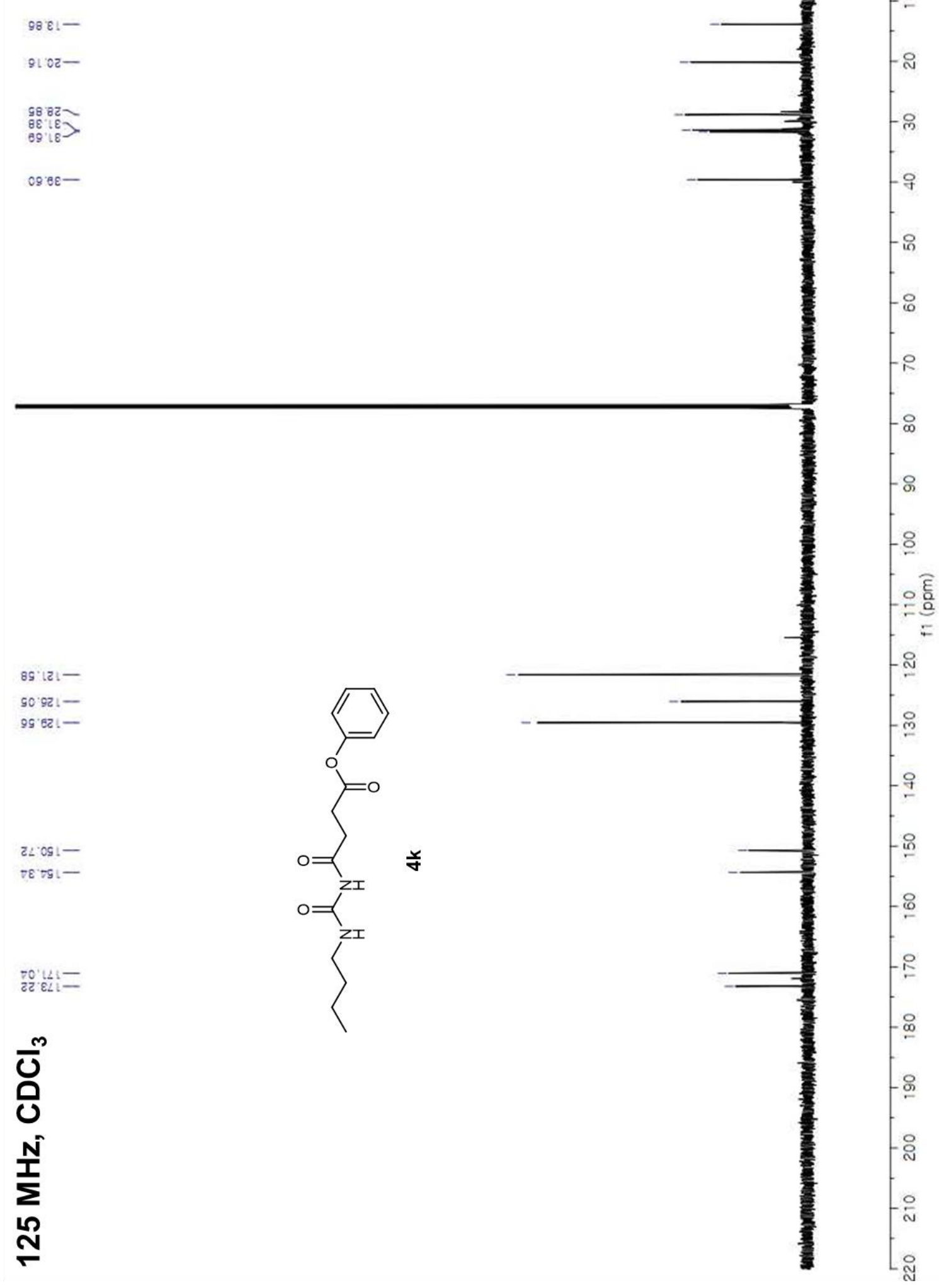




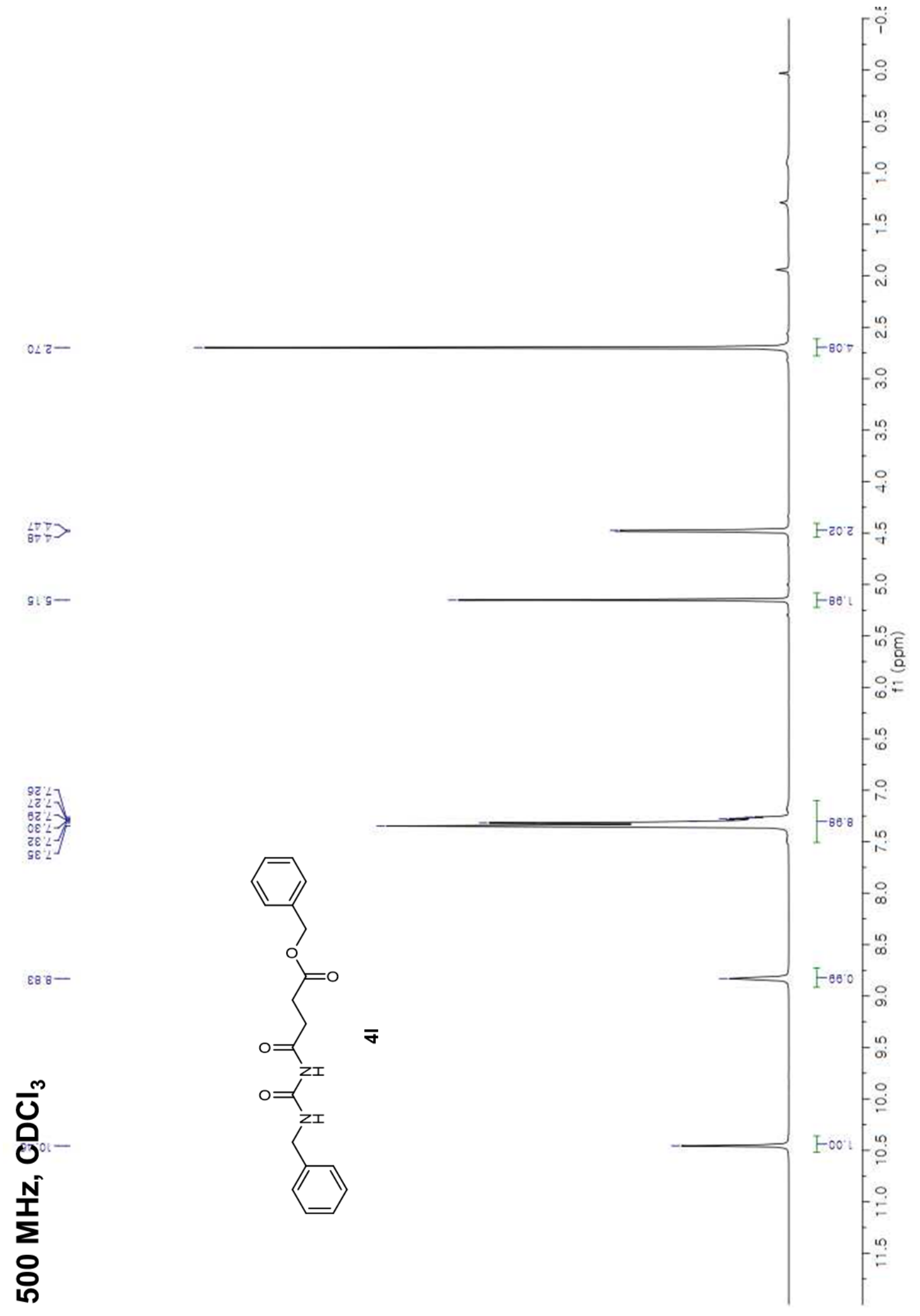




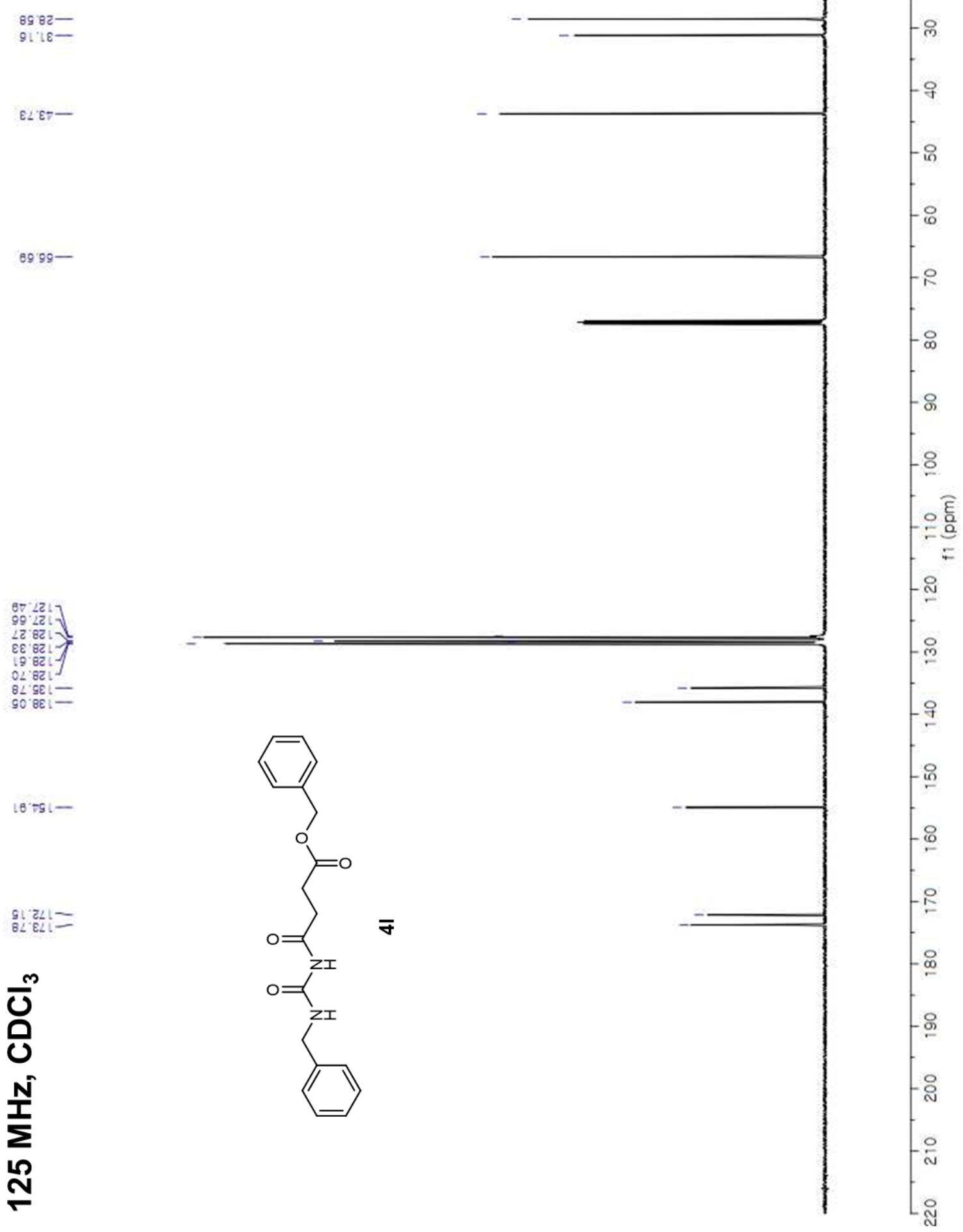




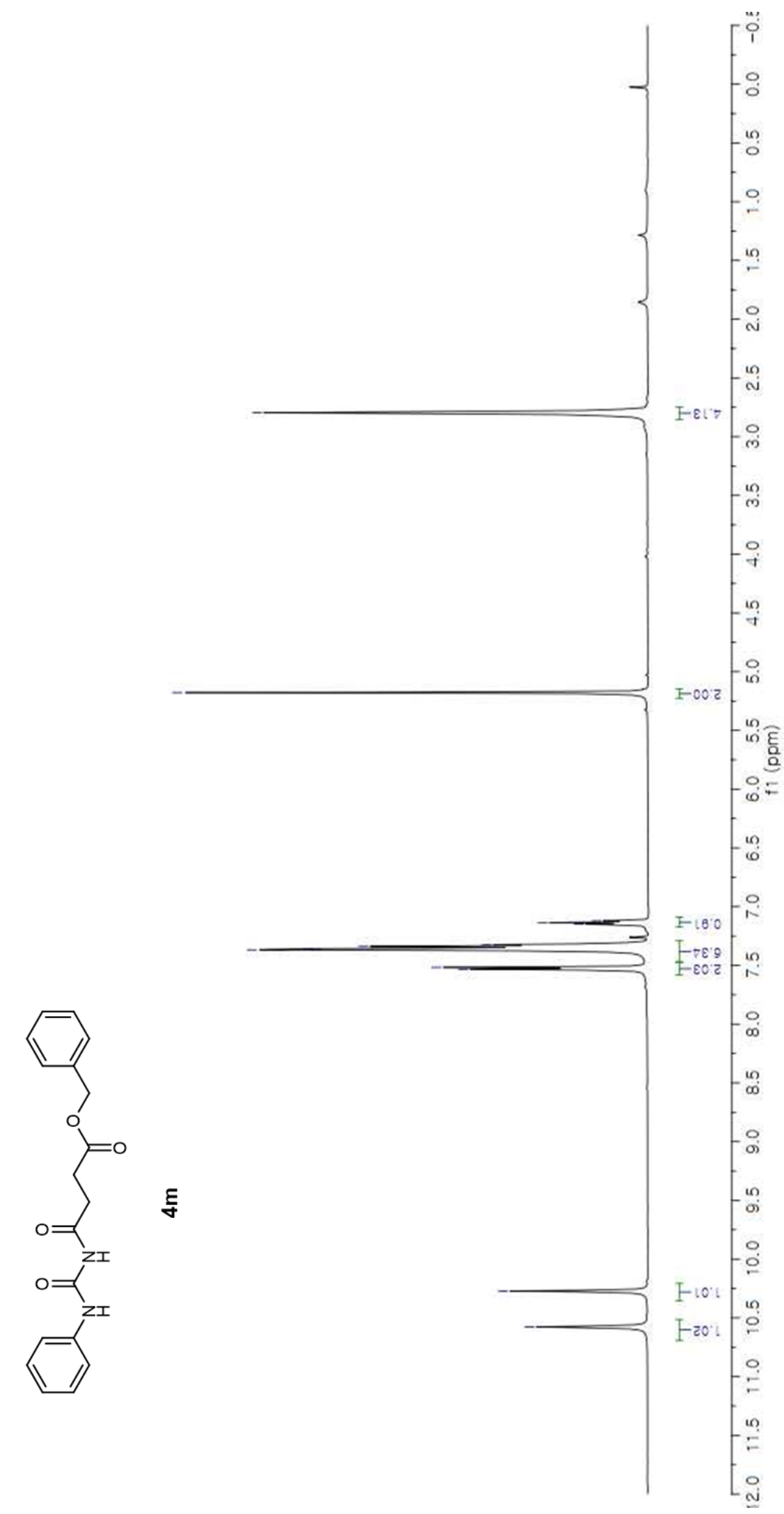

$\frac{m}{0}$
0
ํ.
ํ.
8
0

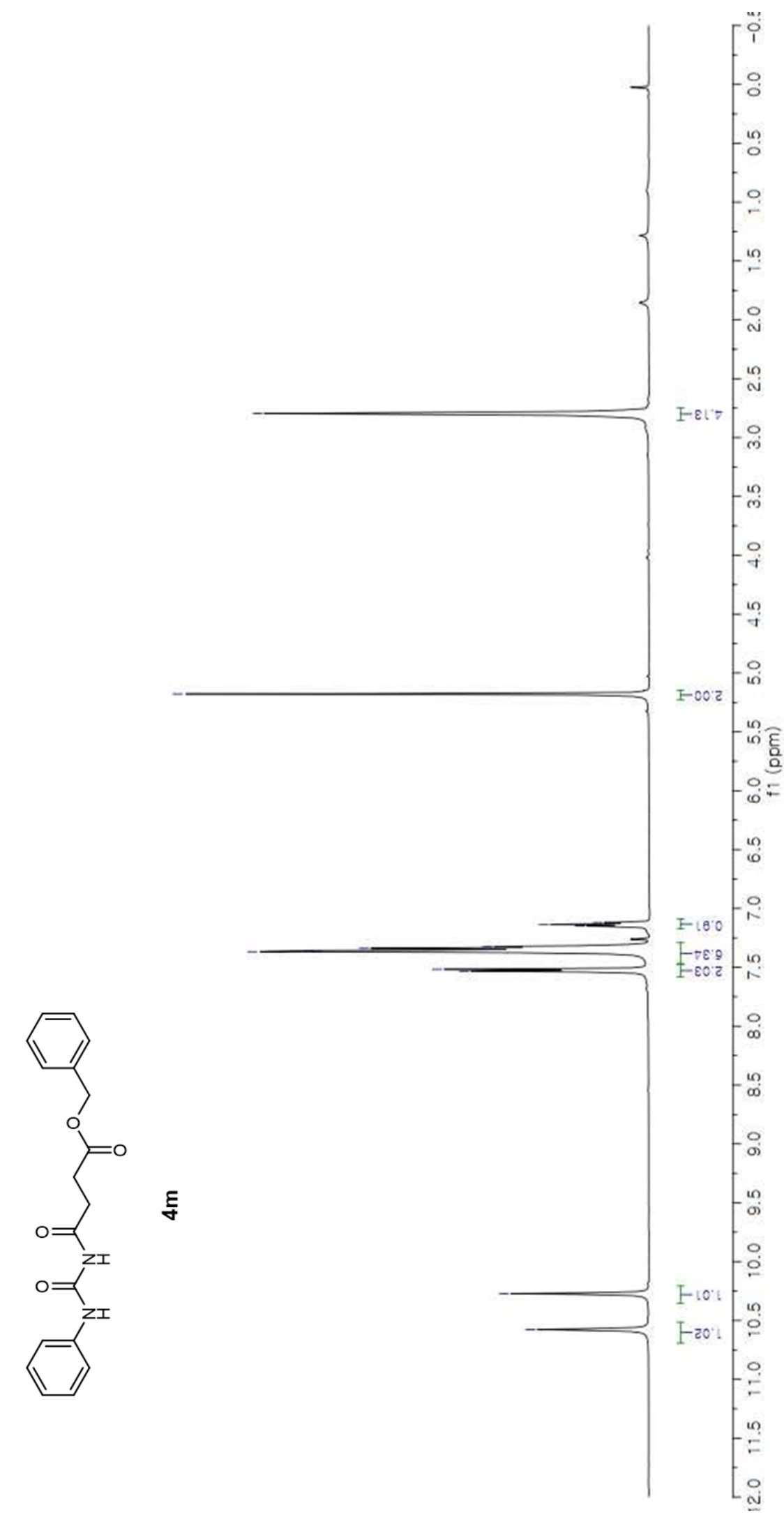

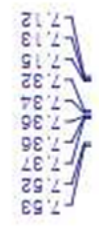


$8982-$

0ั. $18-$

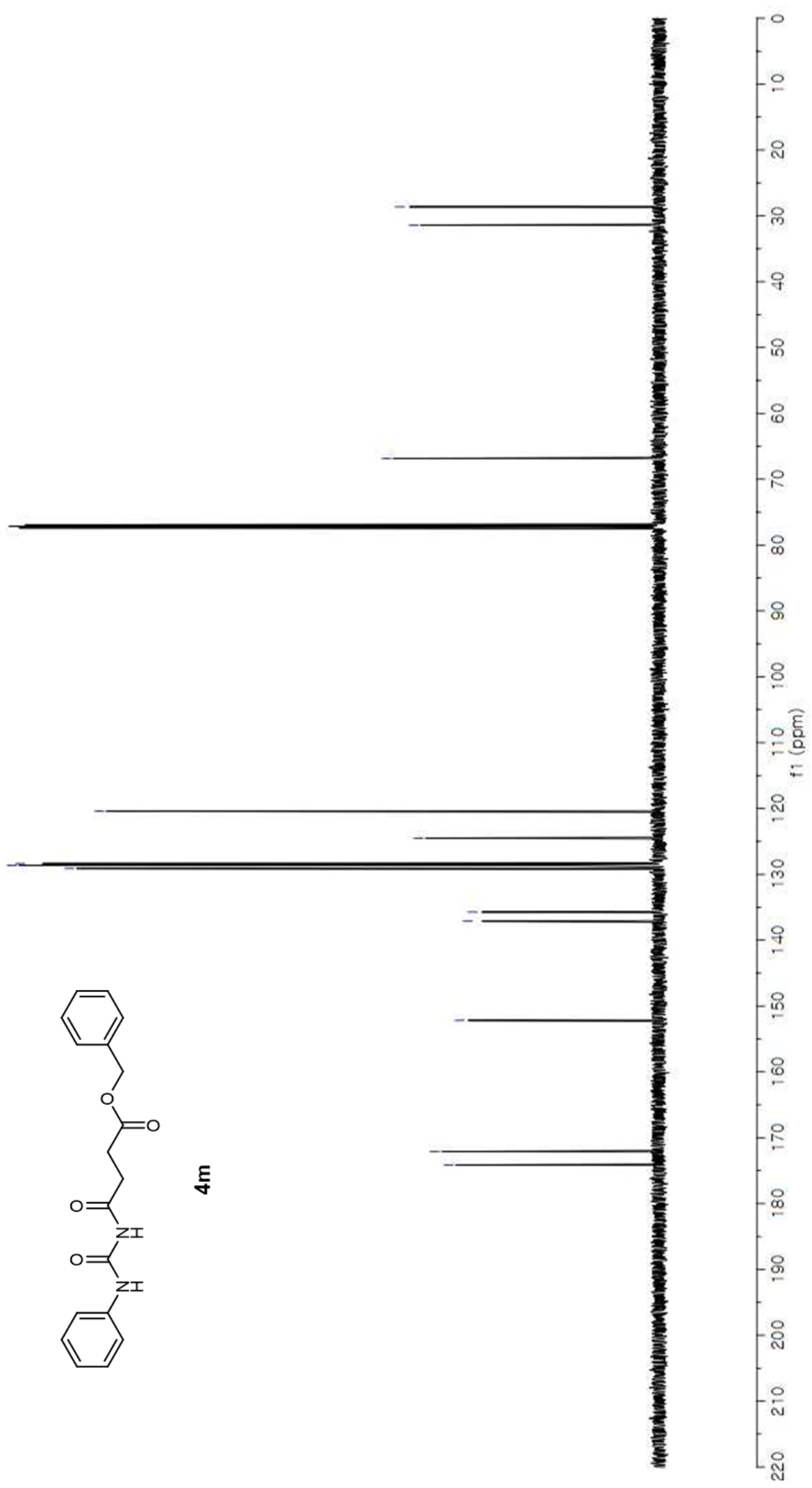

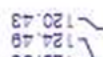

98:82

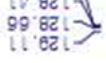

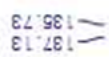

6. $29:-$

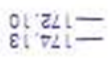

তับ

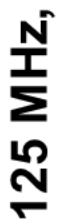




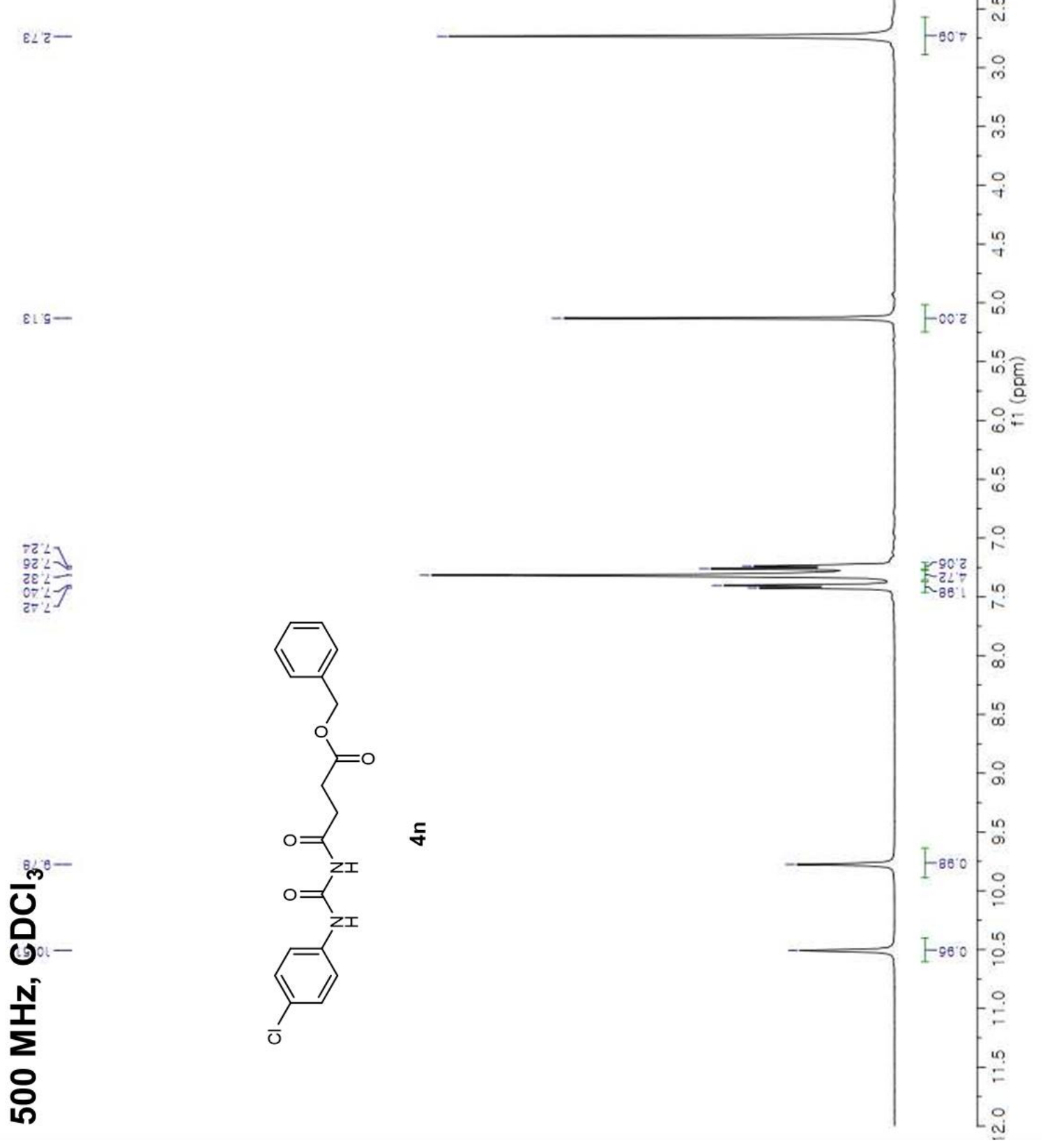




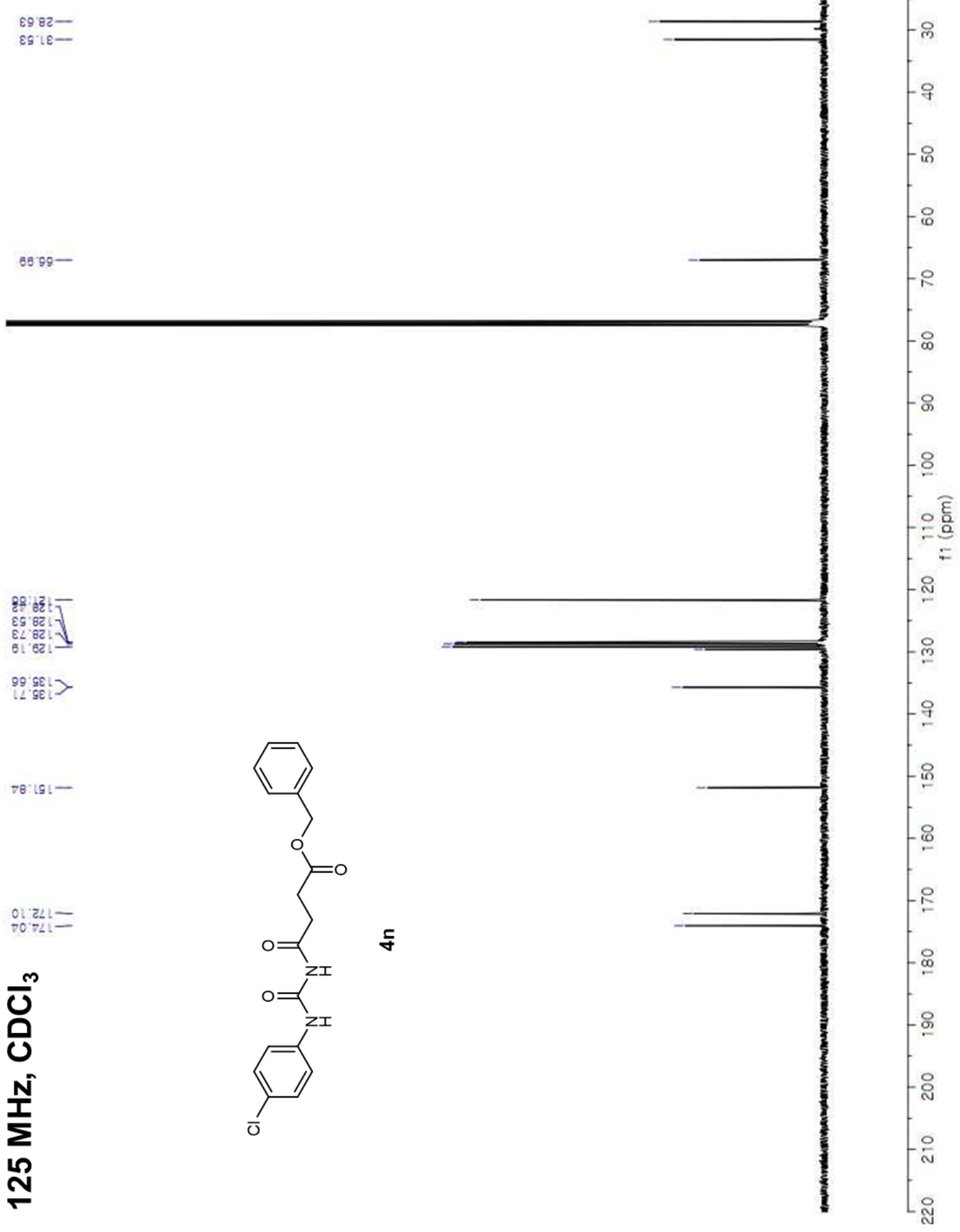




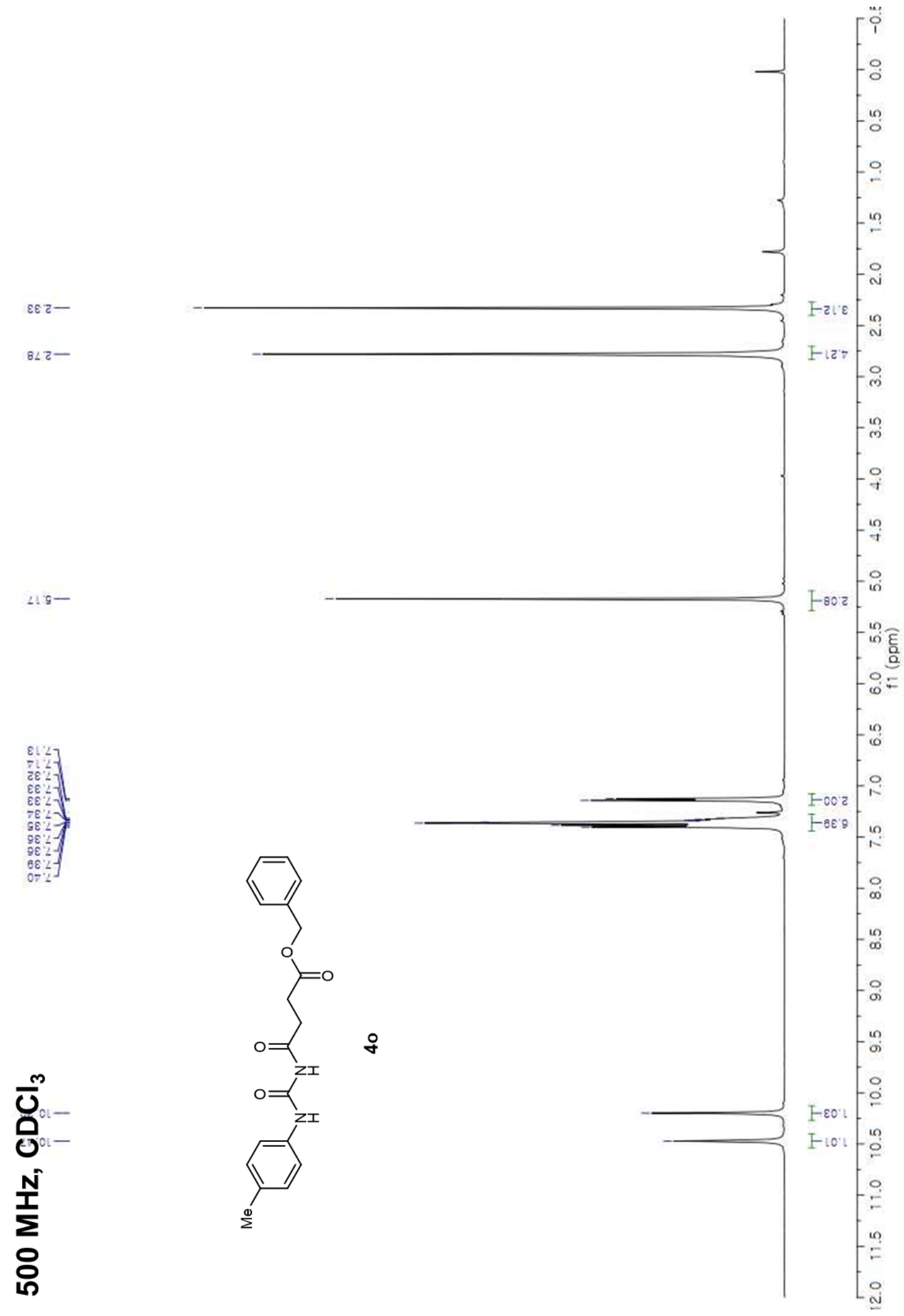




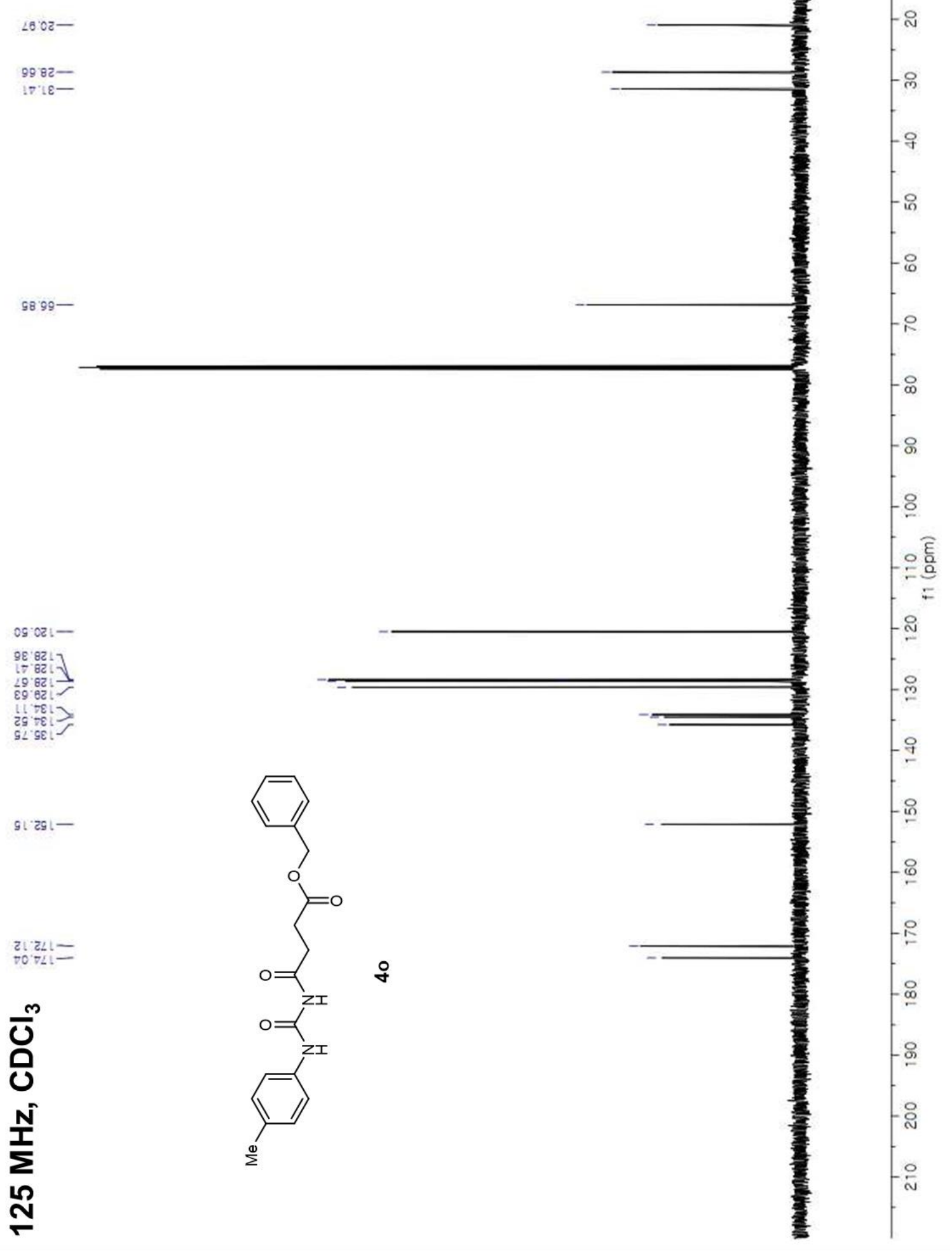




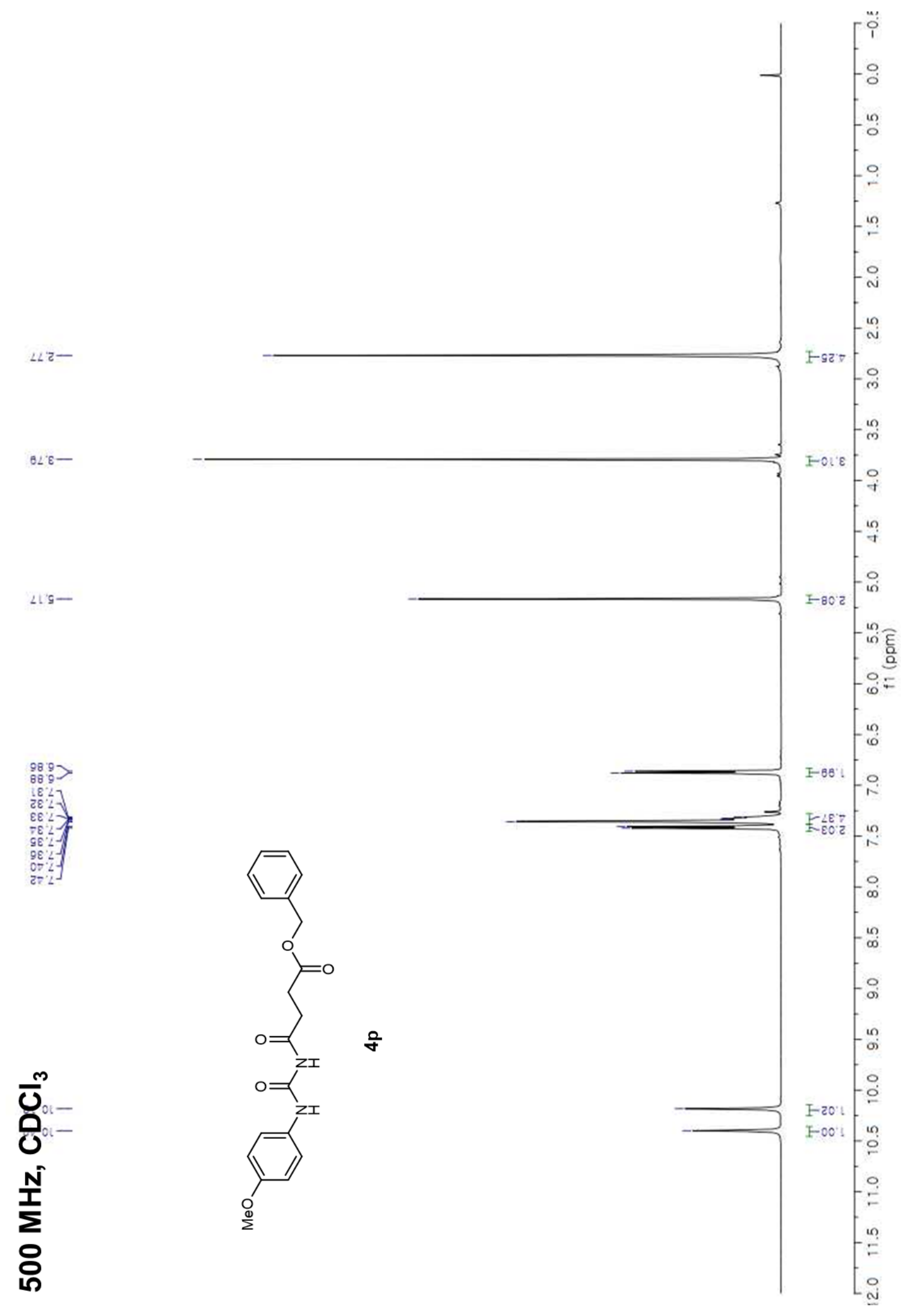




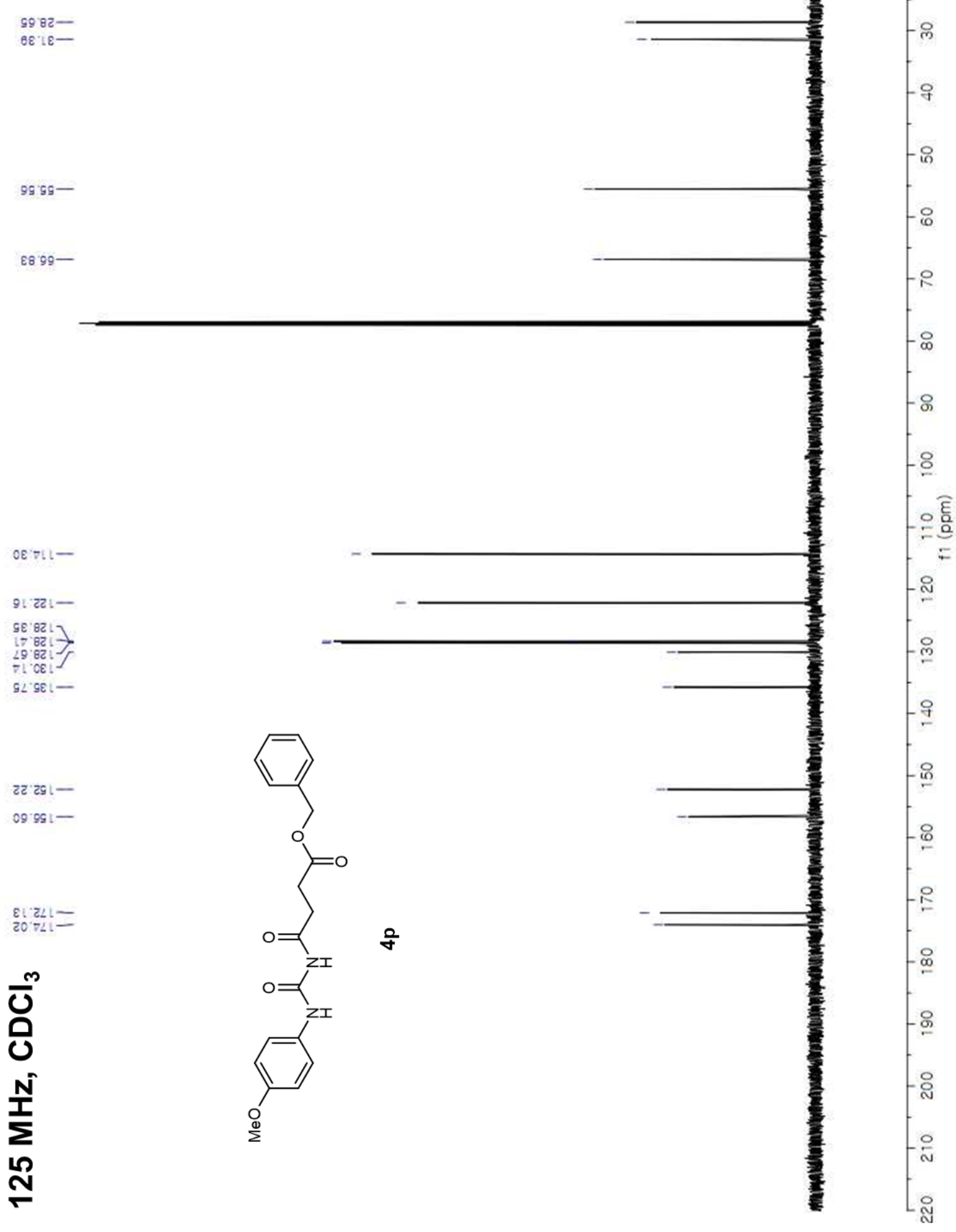



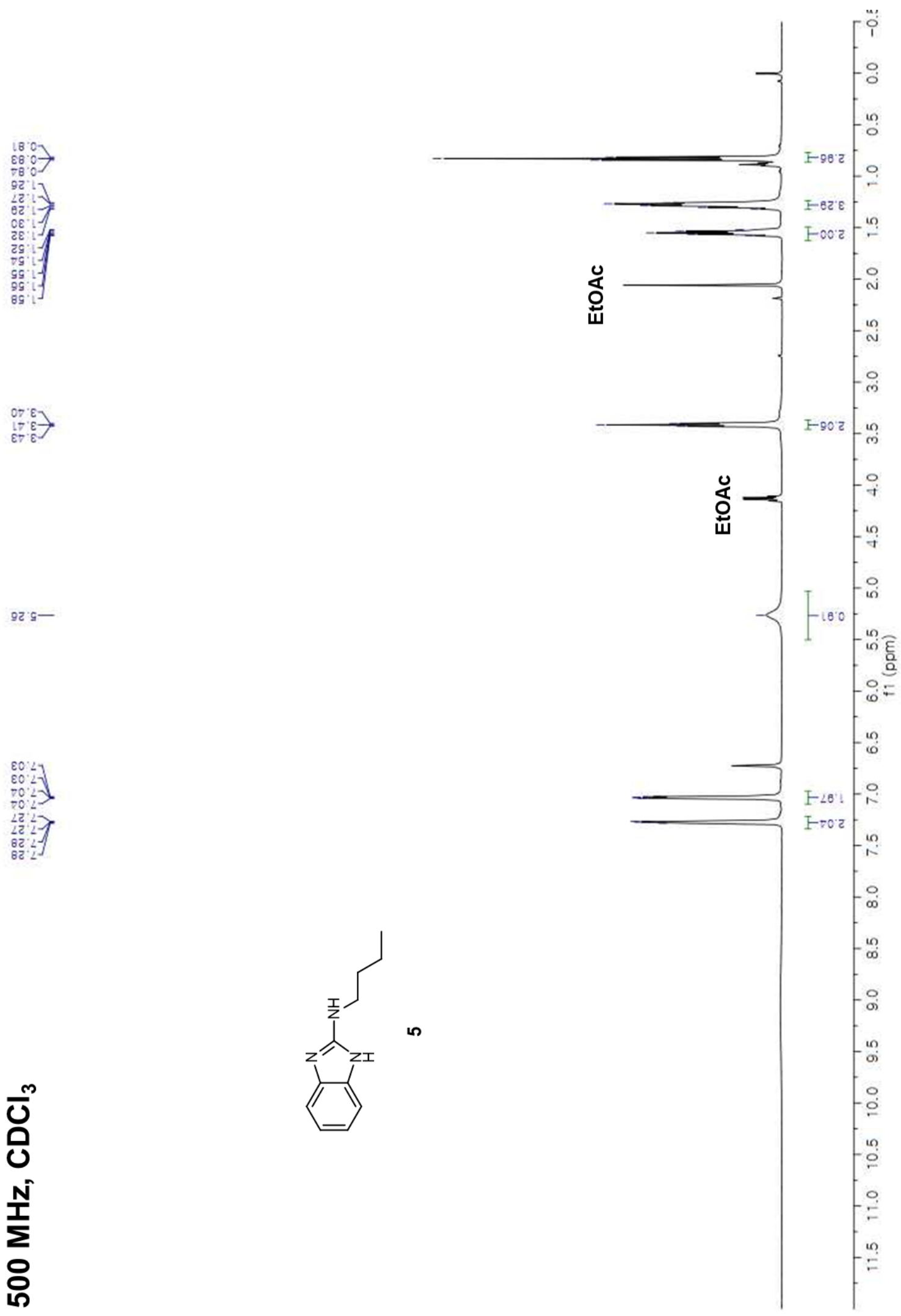

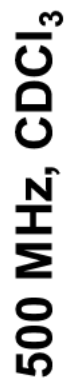



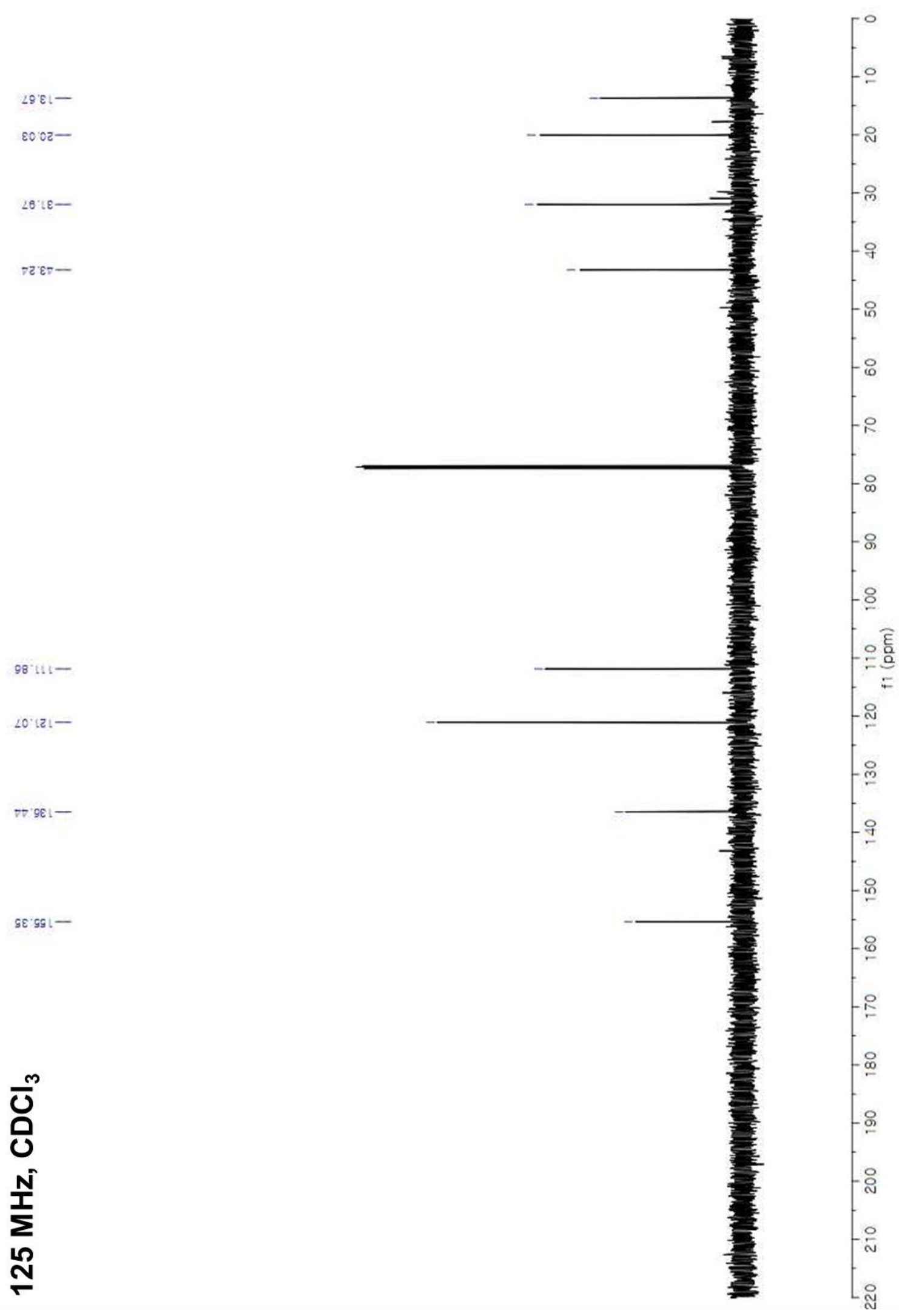


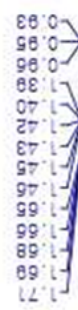

aryeg

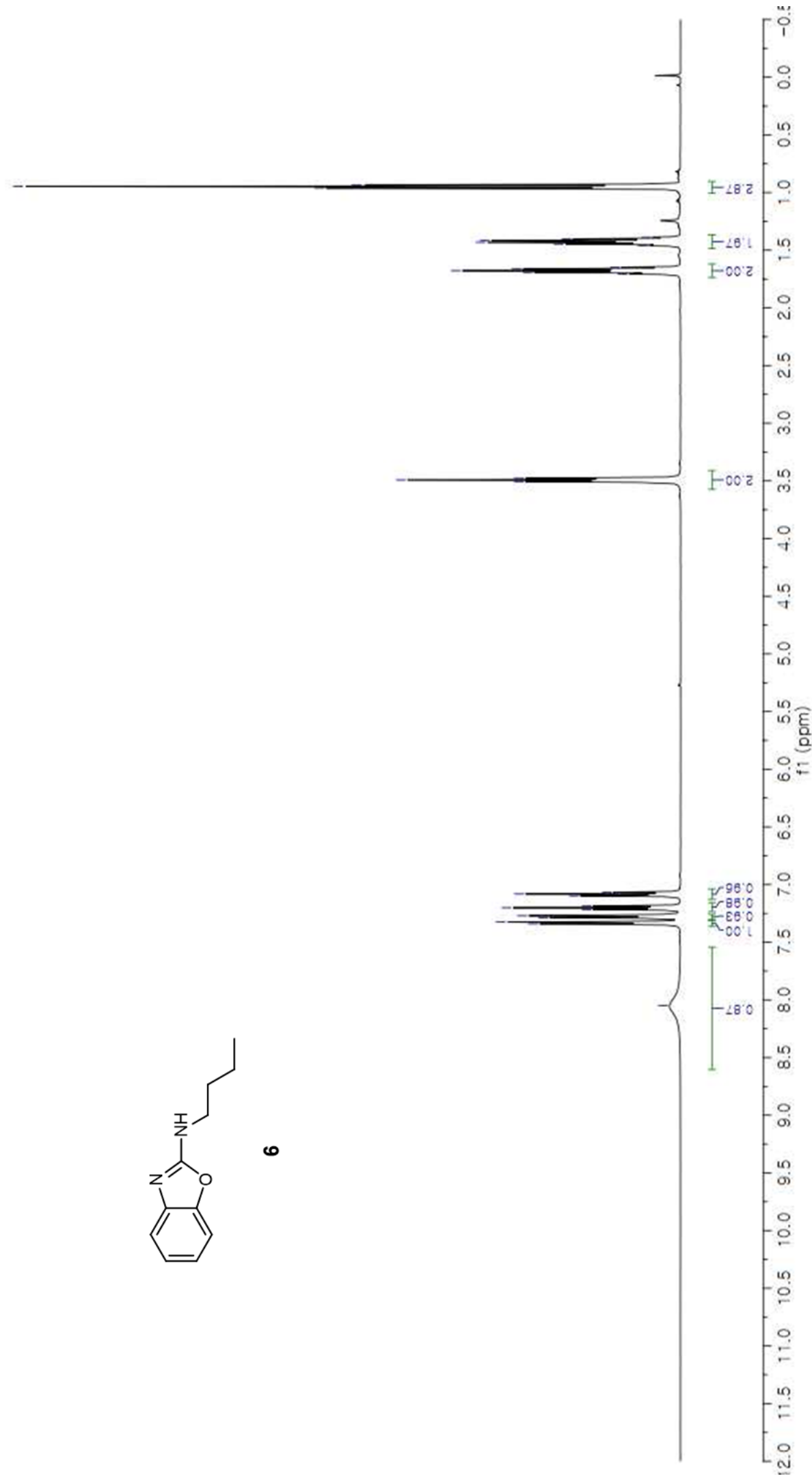

$\frac{m}{0}$
0
N
ํ.
0
0 


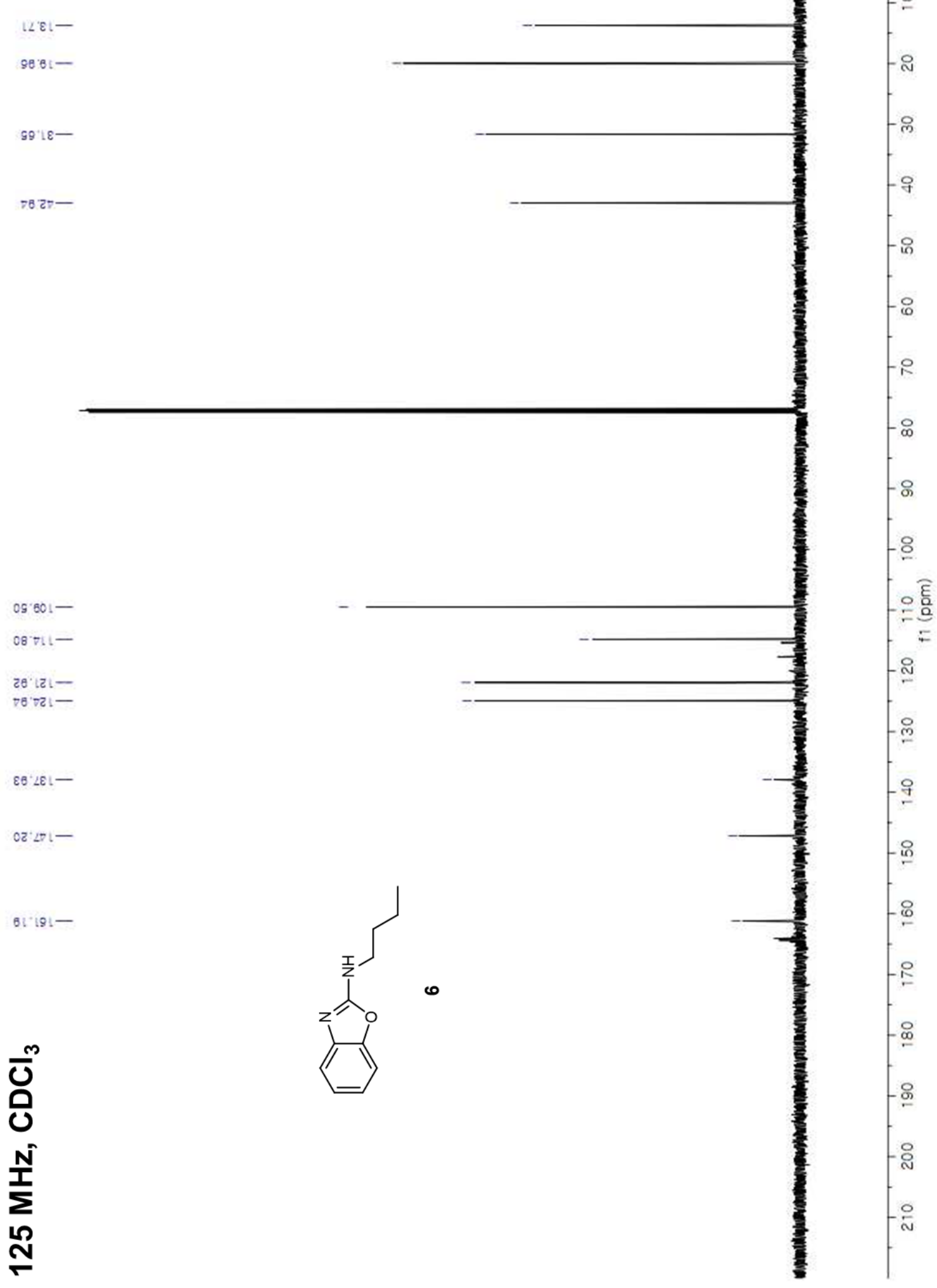




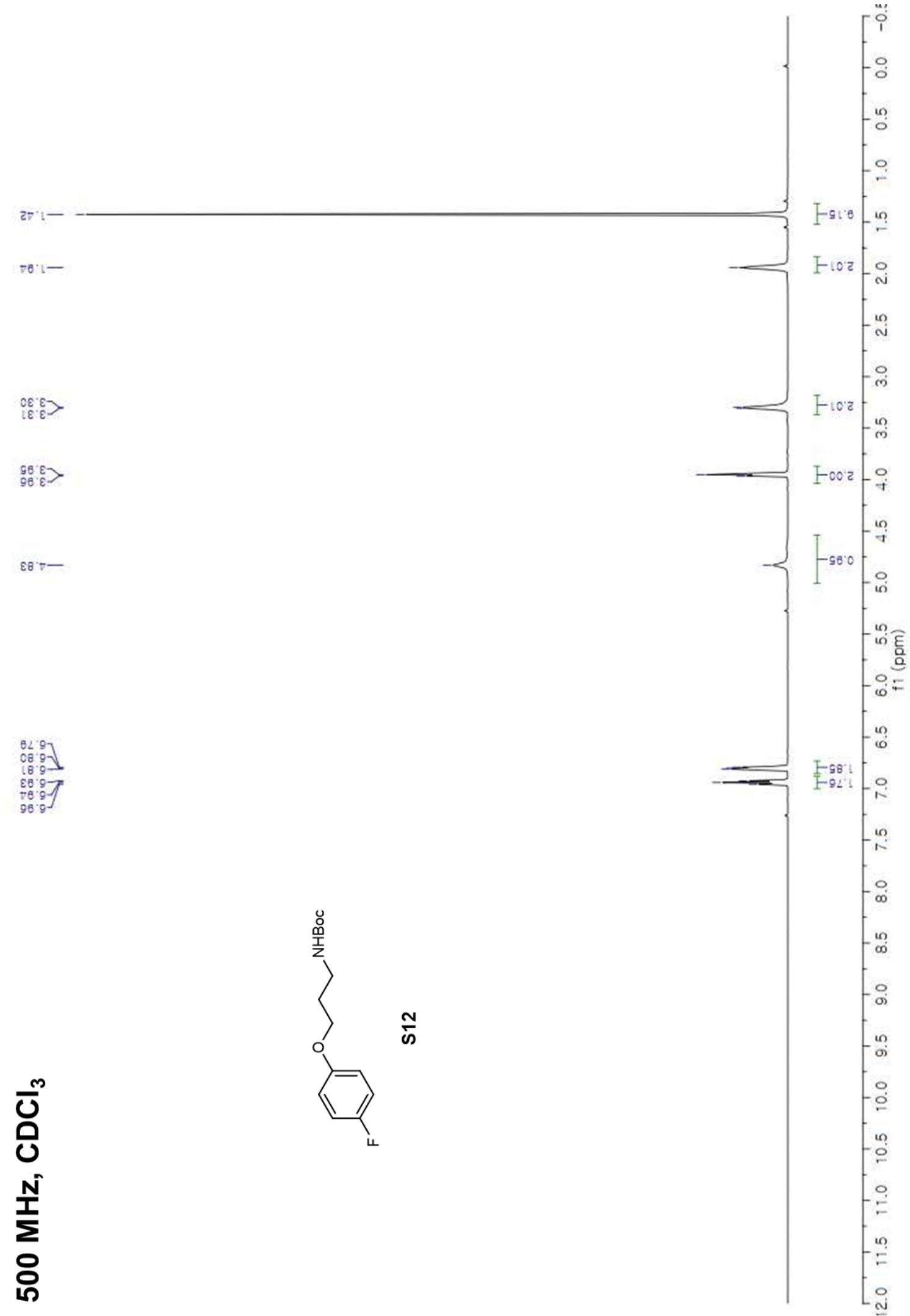




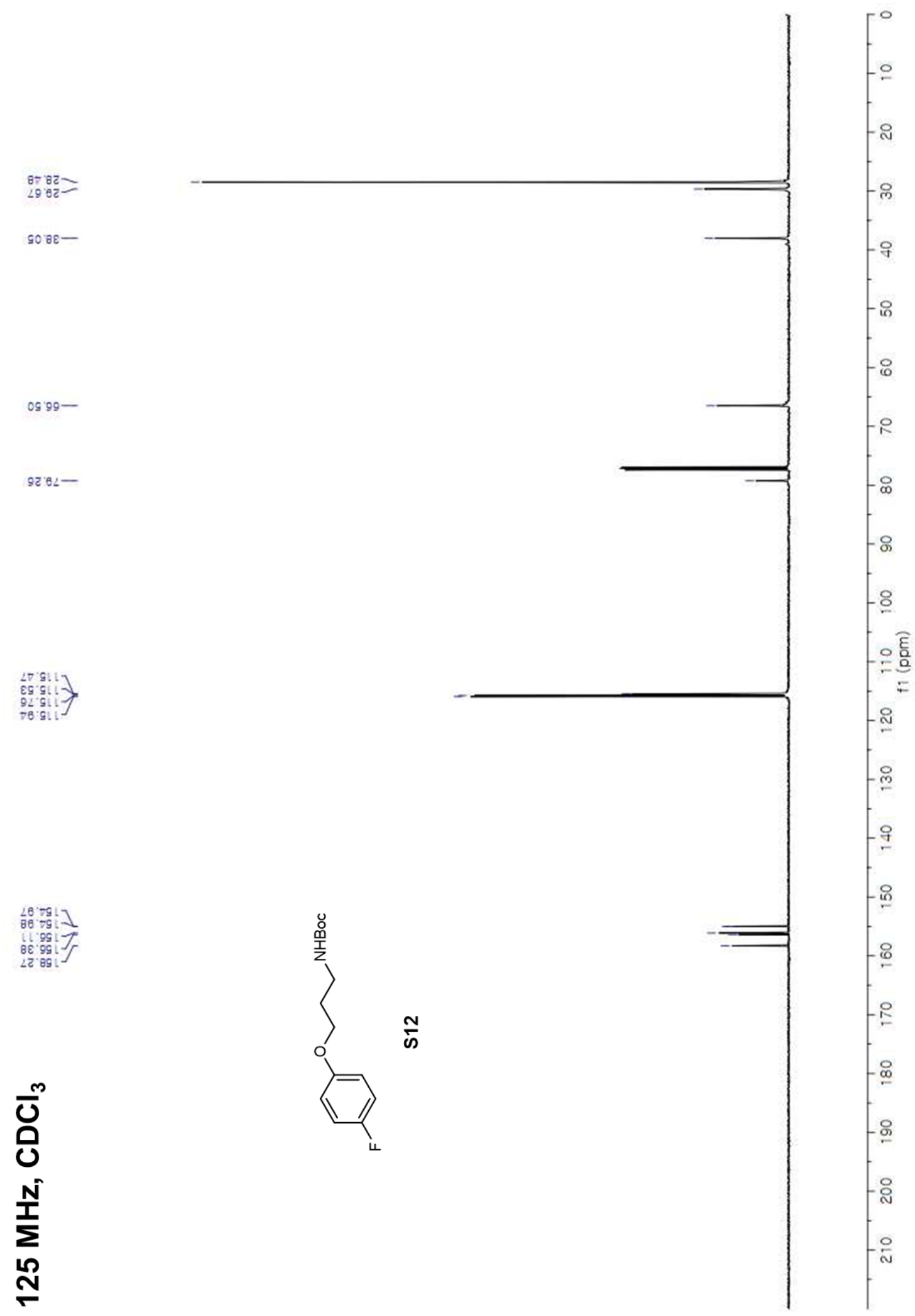




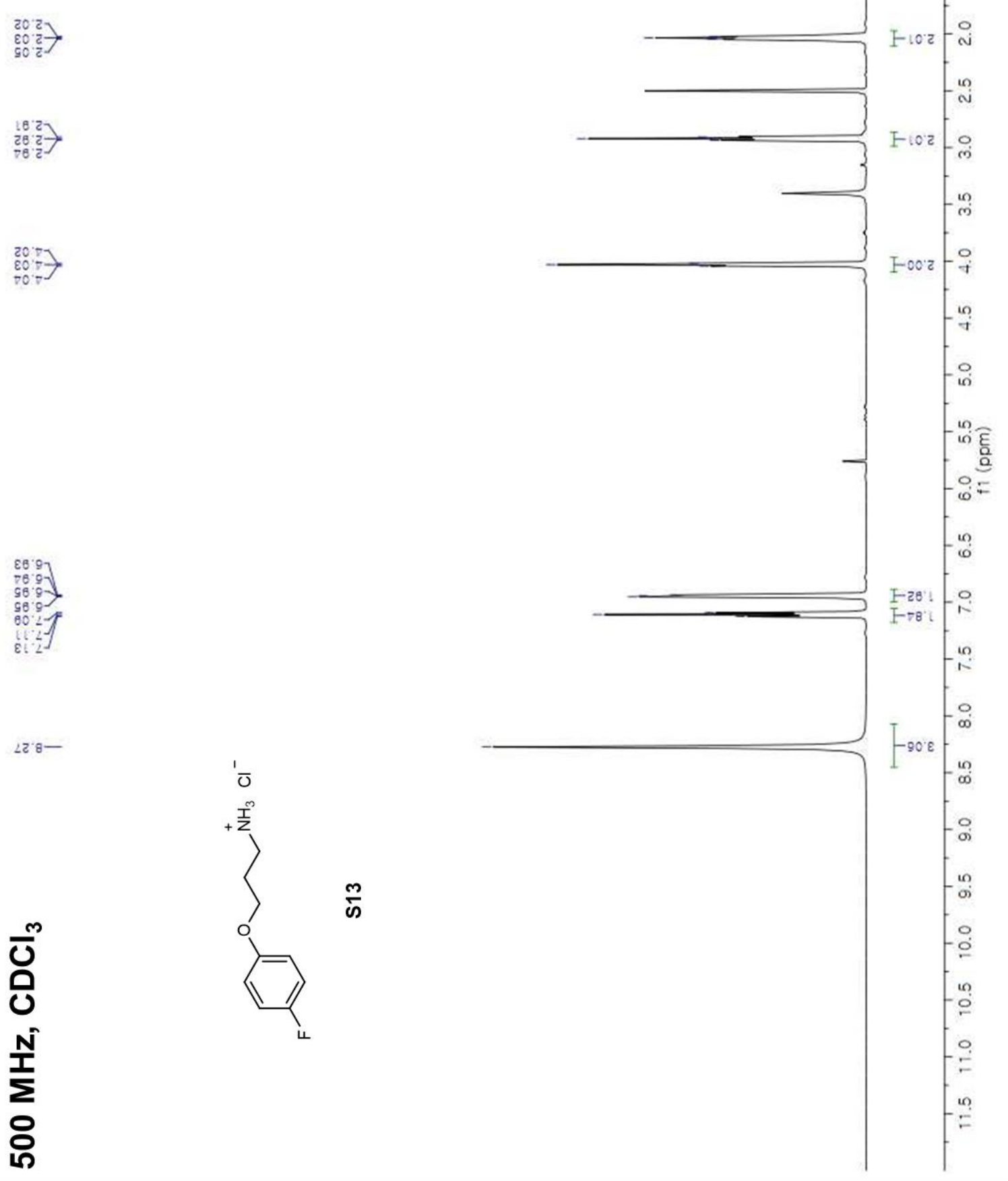




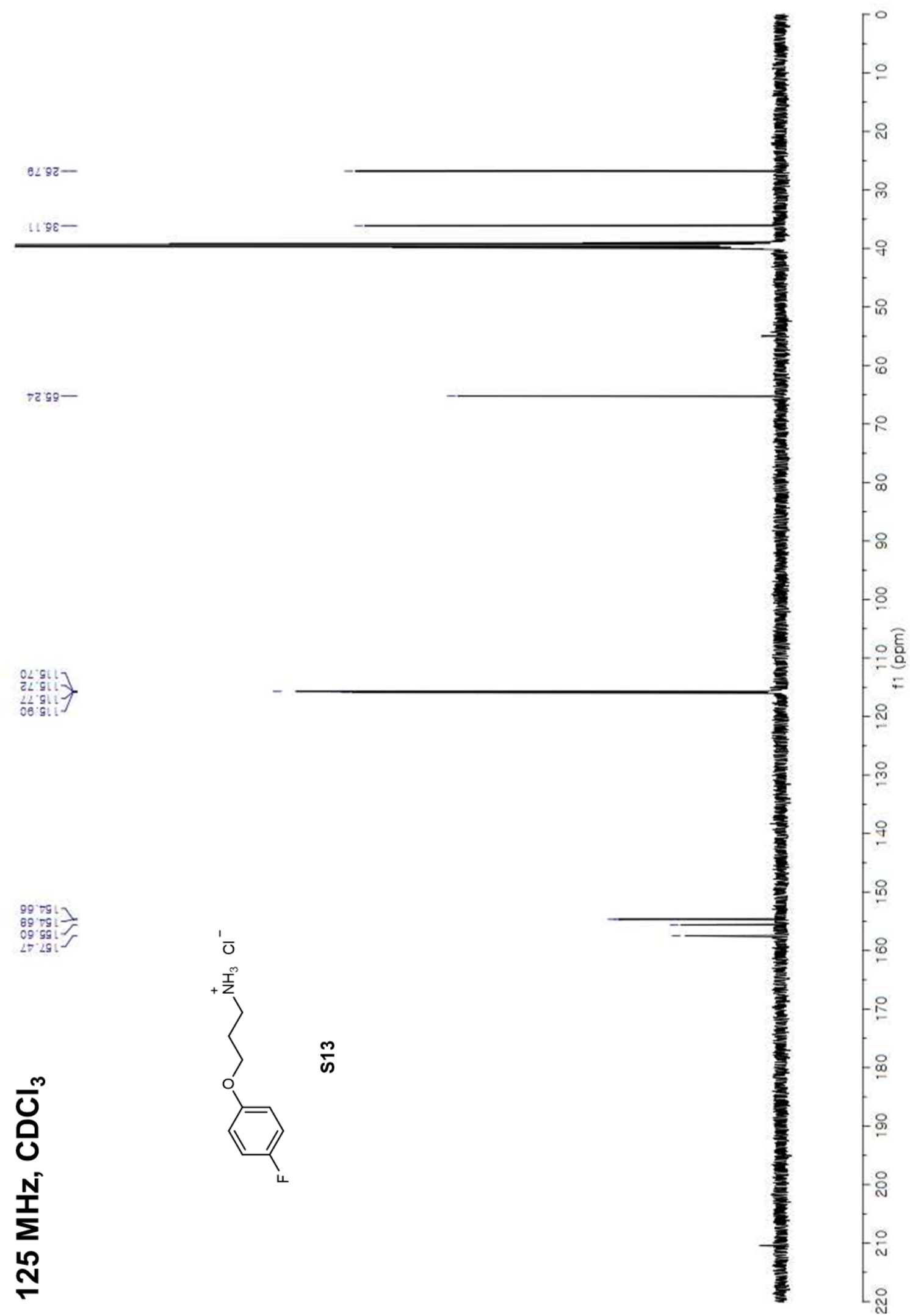




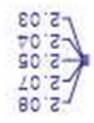

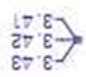

急此

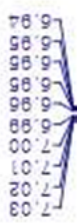

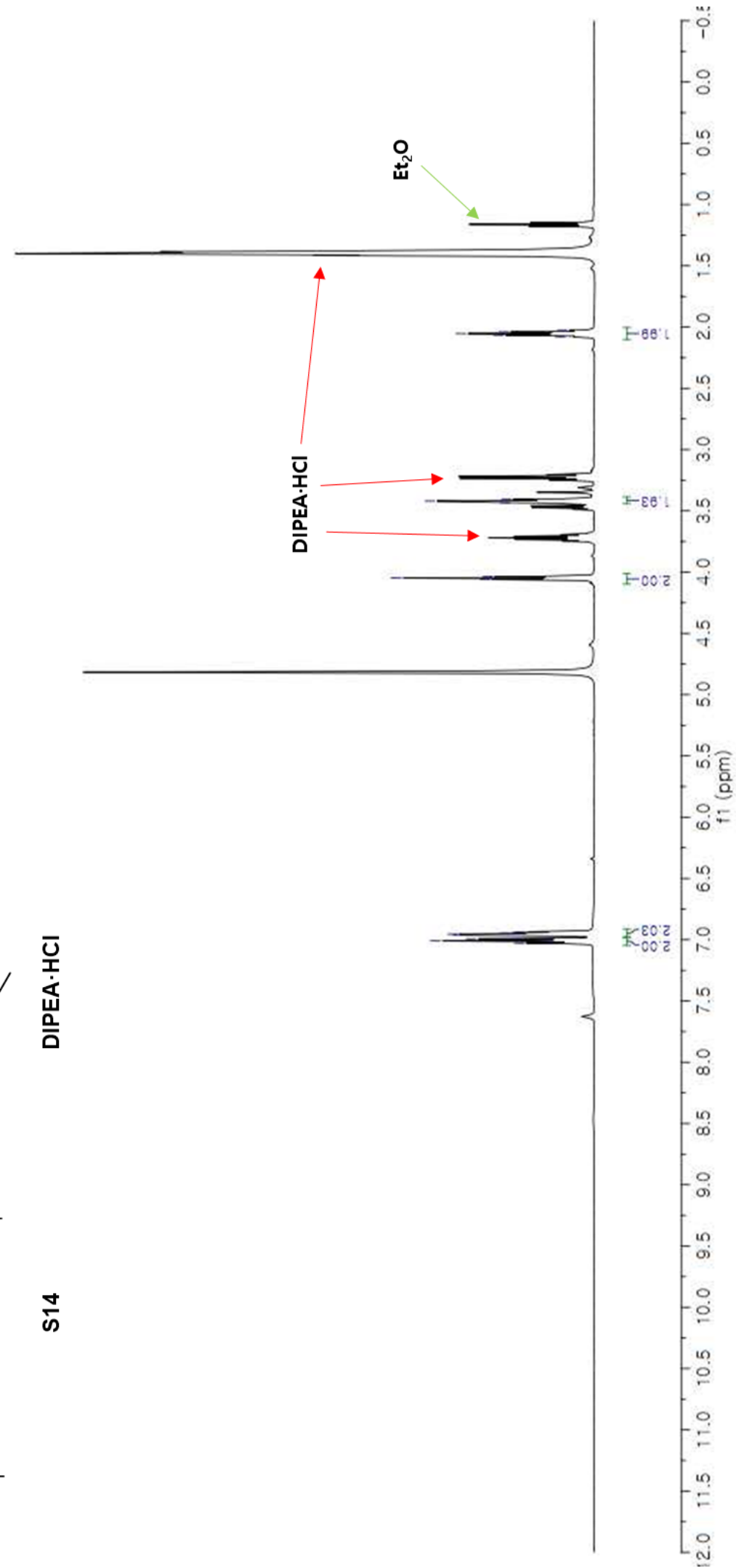




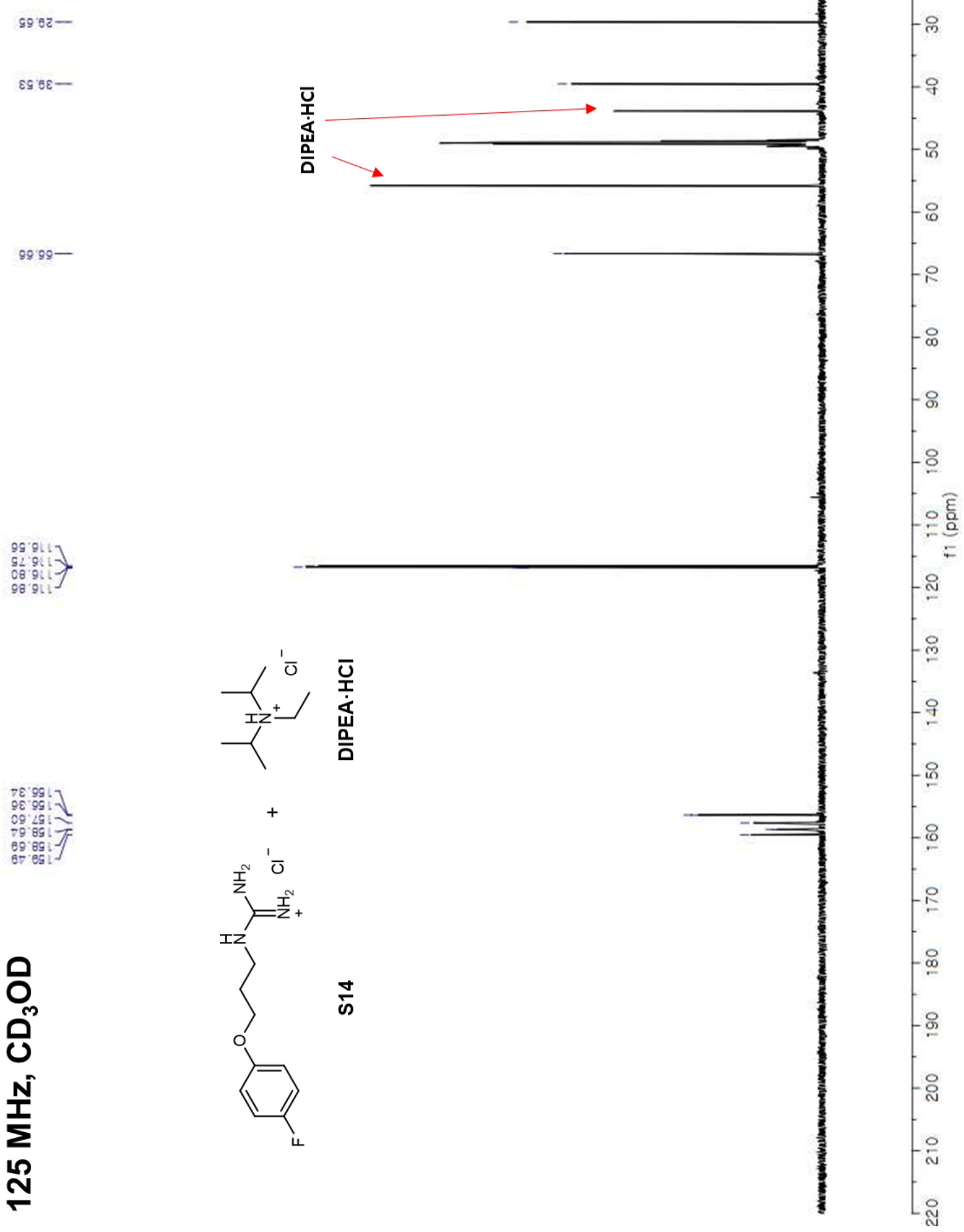




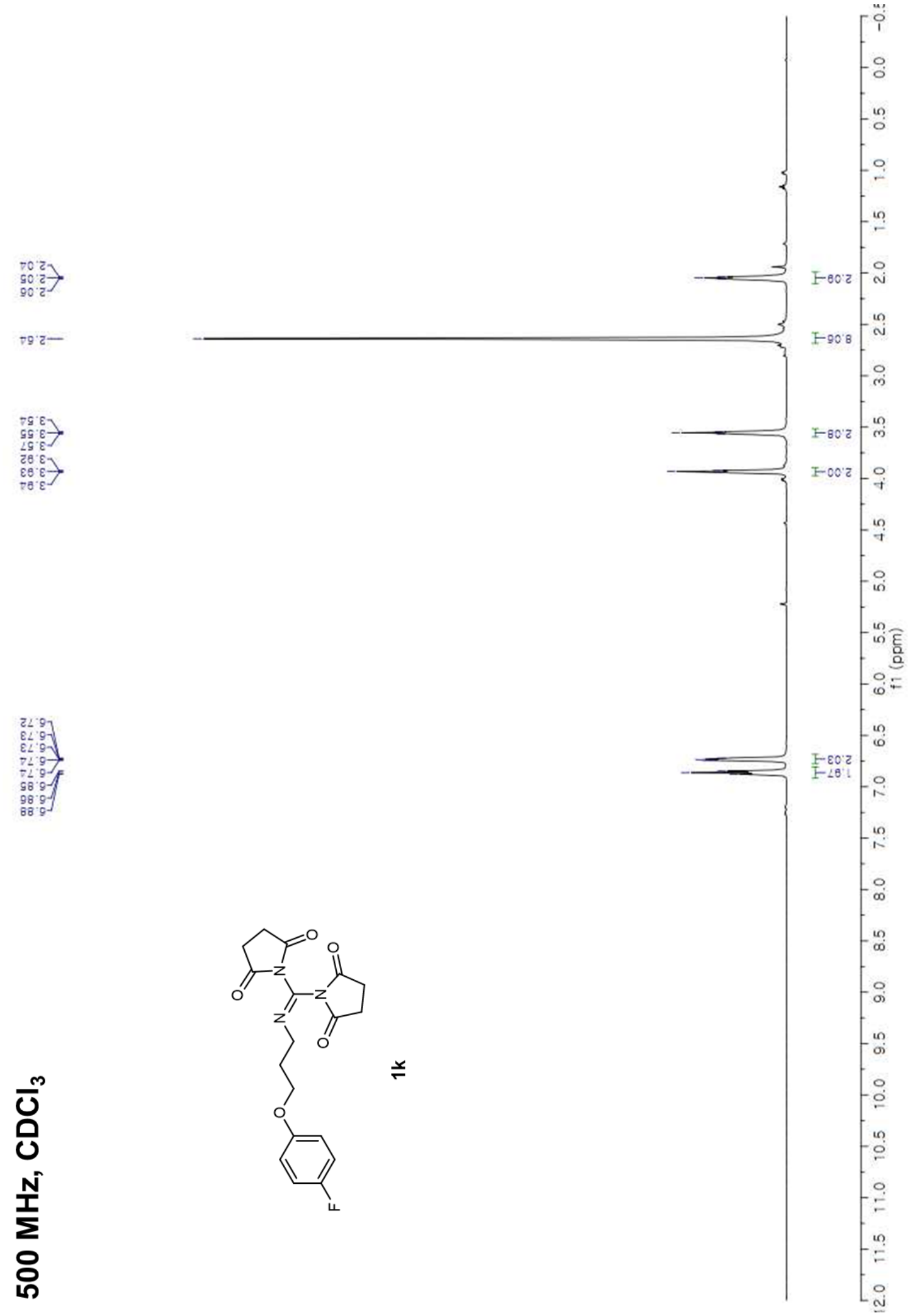




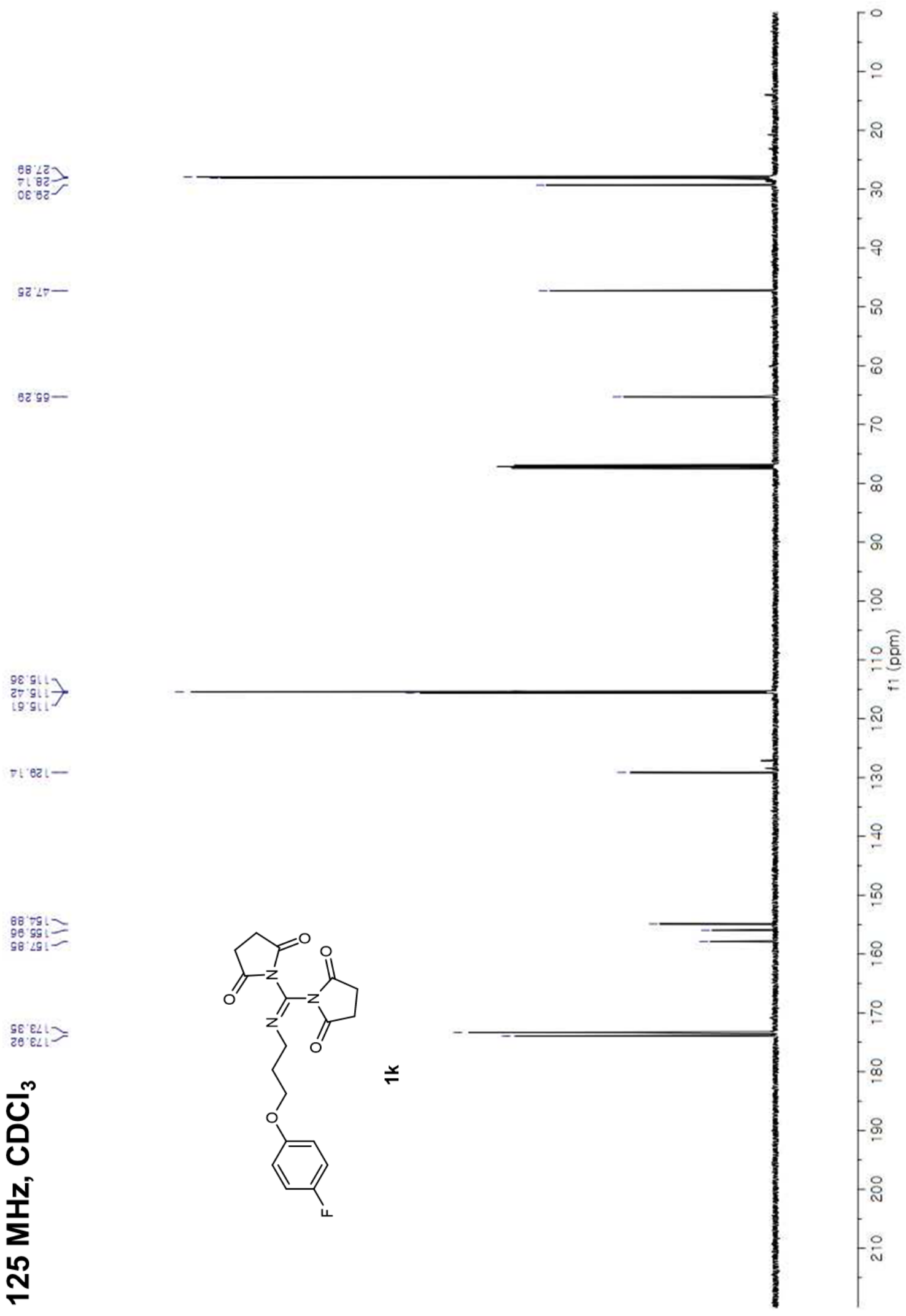



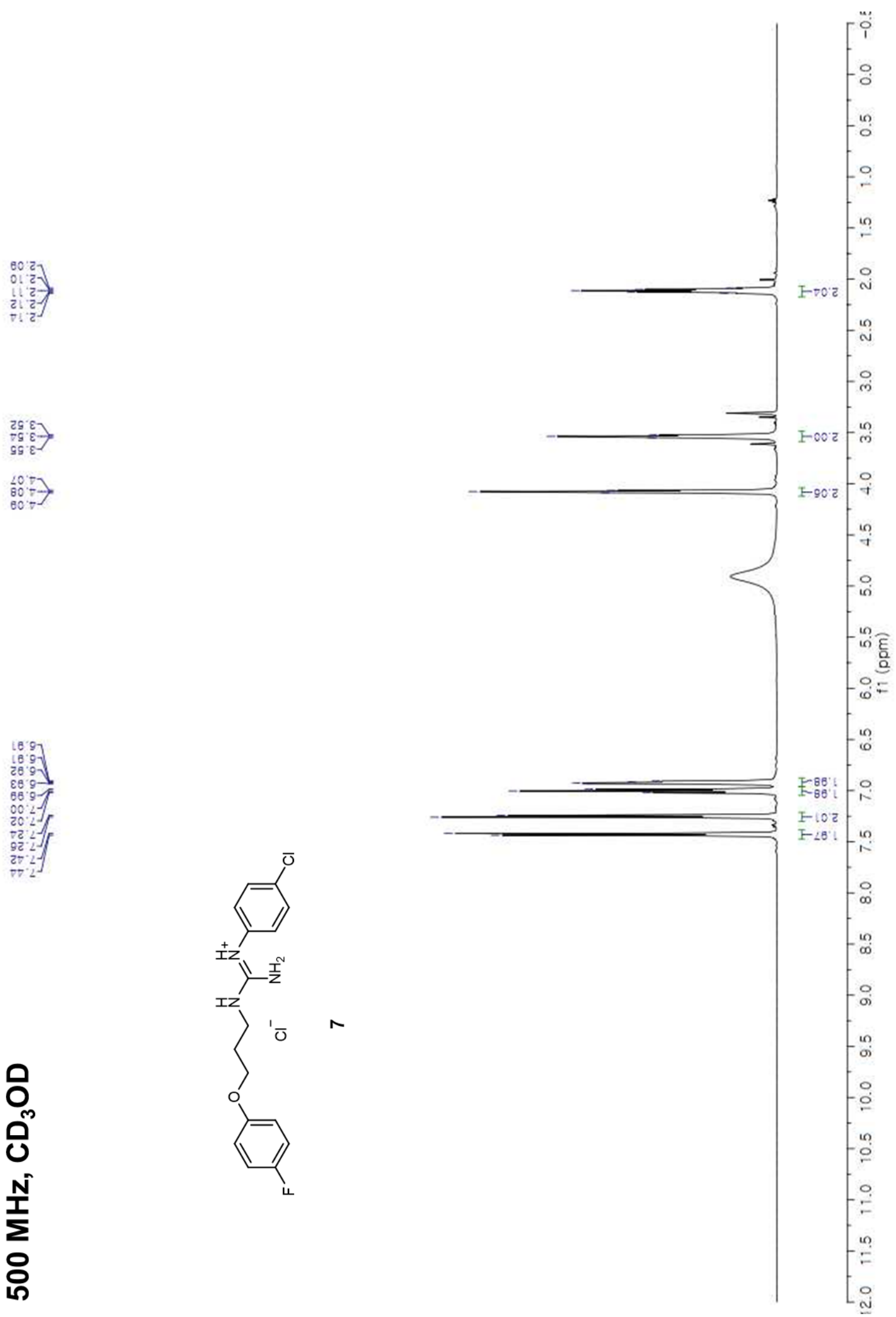


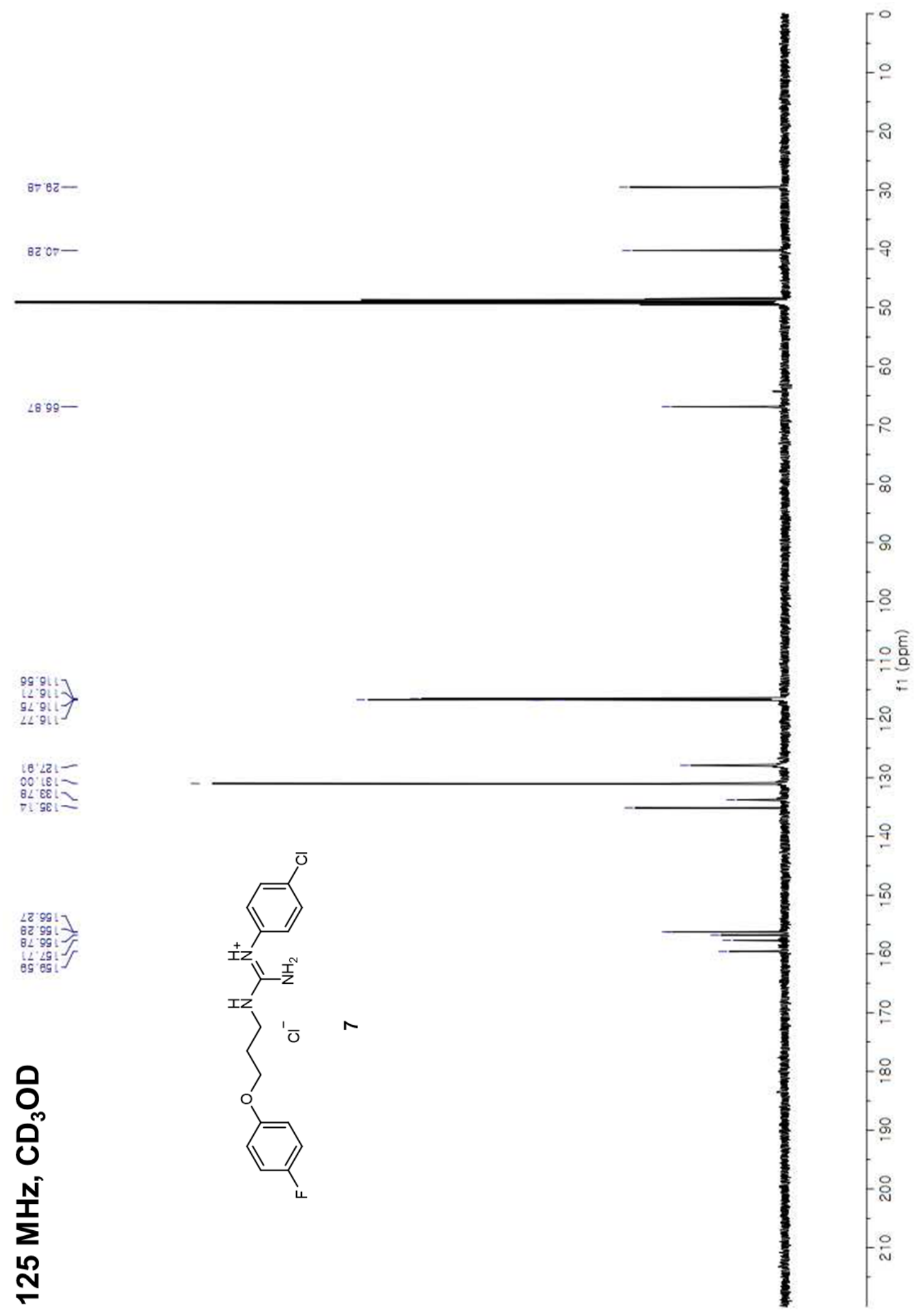




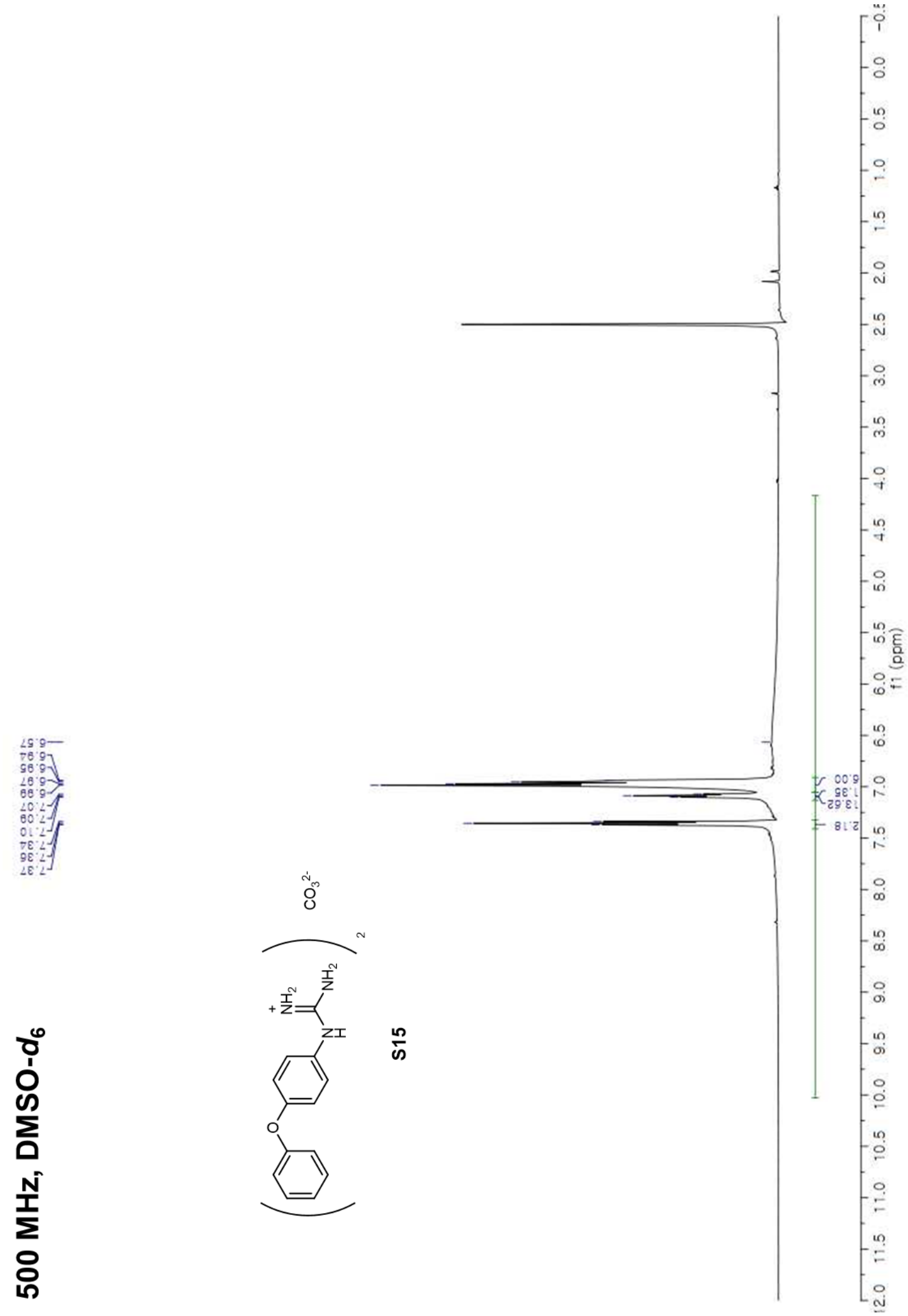




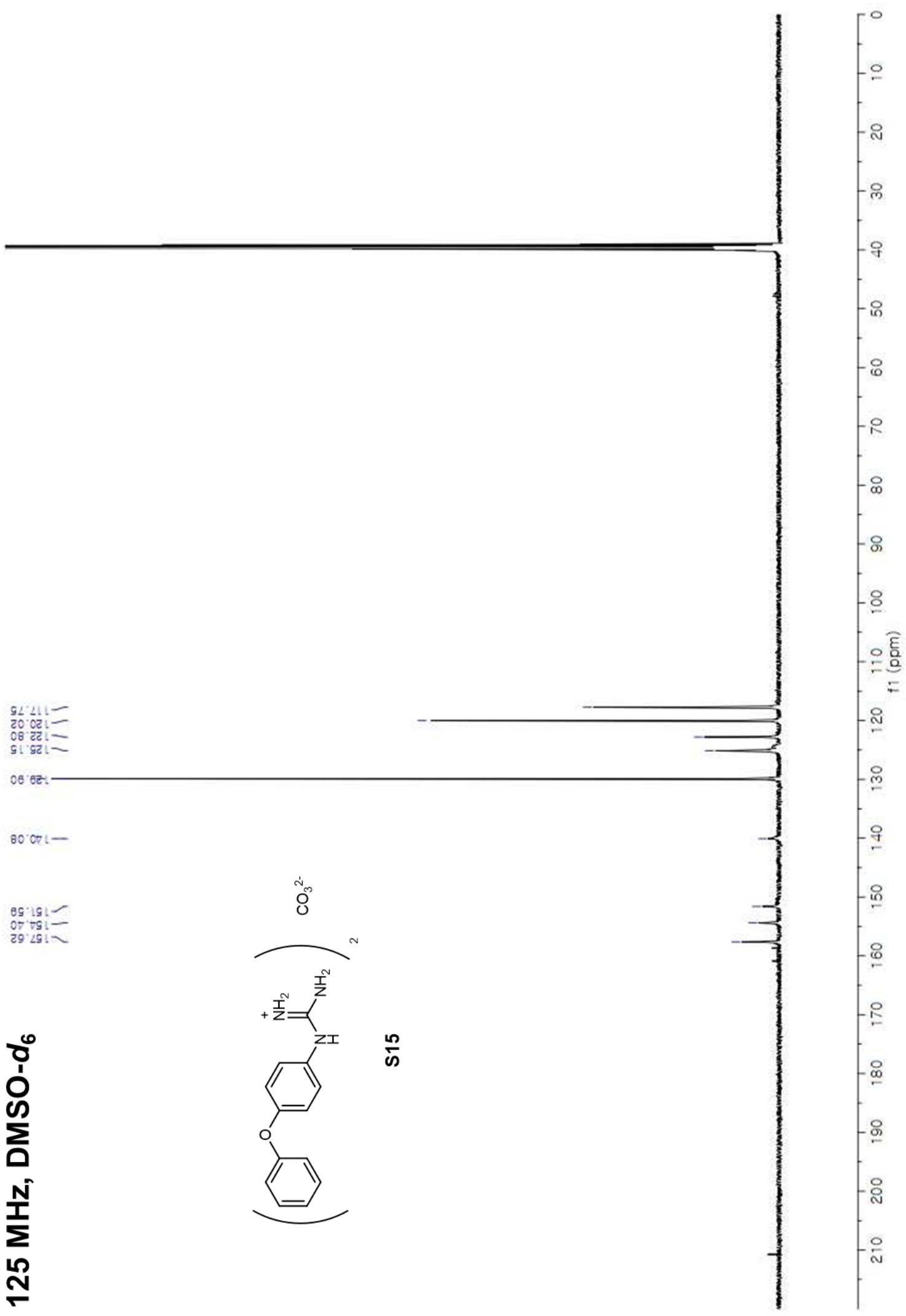




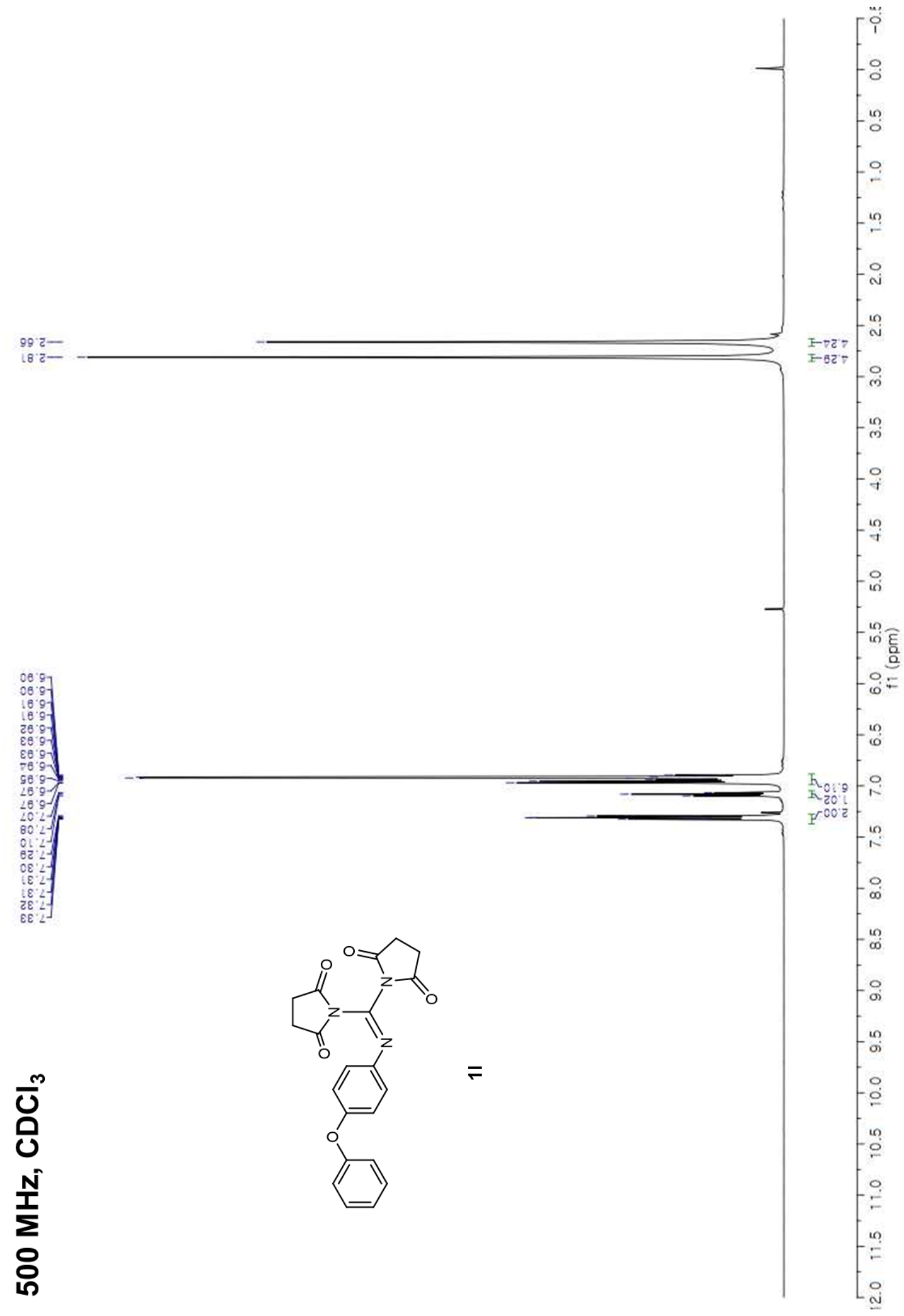


$0 z \varepsilon z$
$\varepsilon \varepsilon \theta \varepsilon$

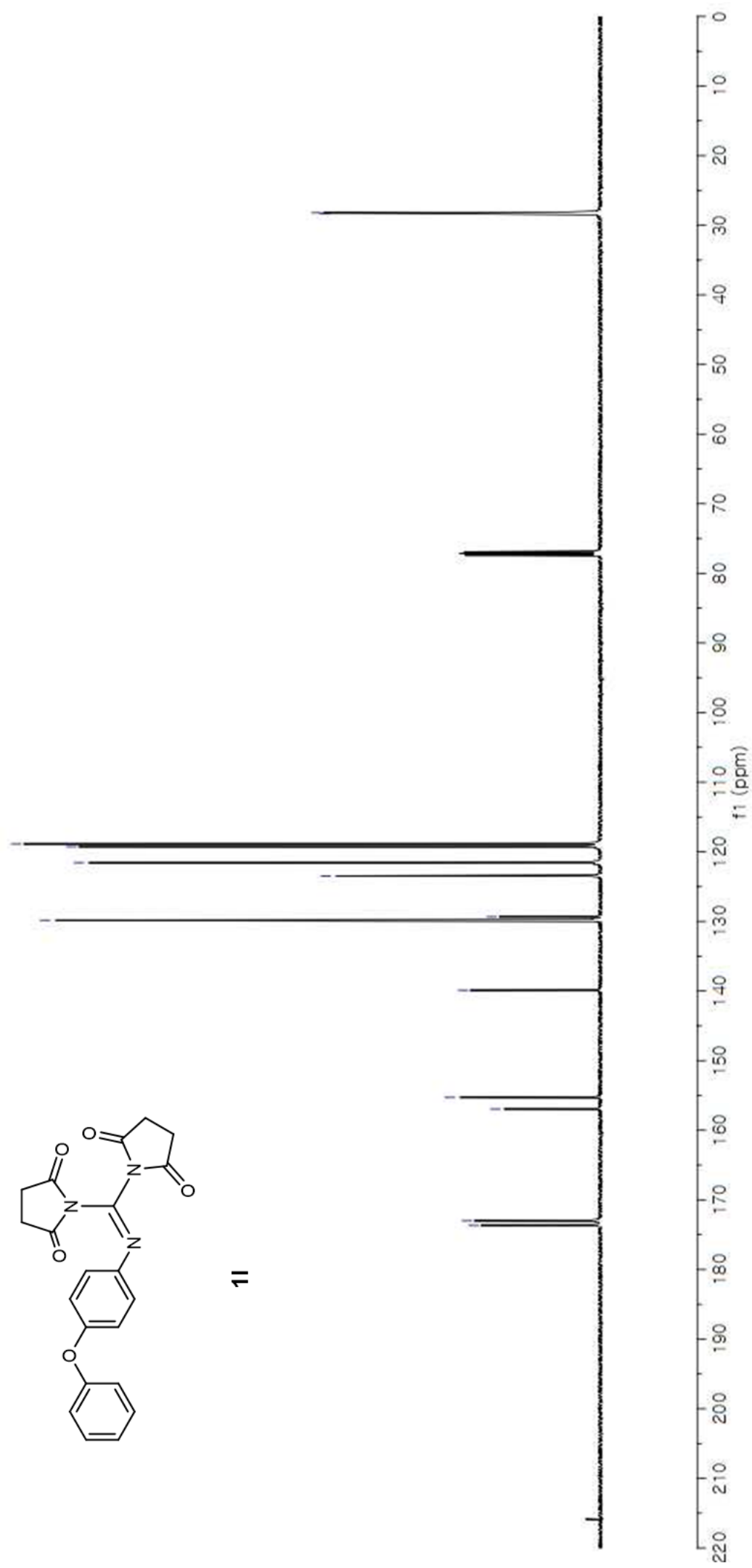



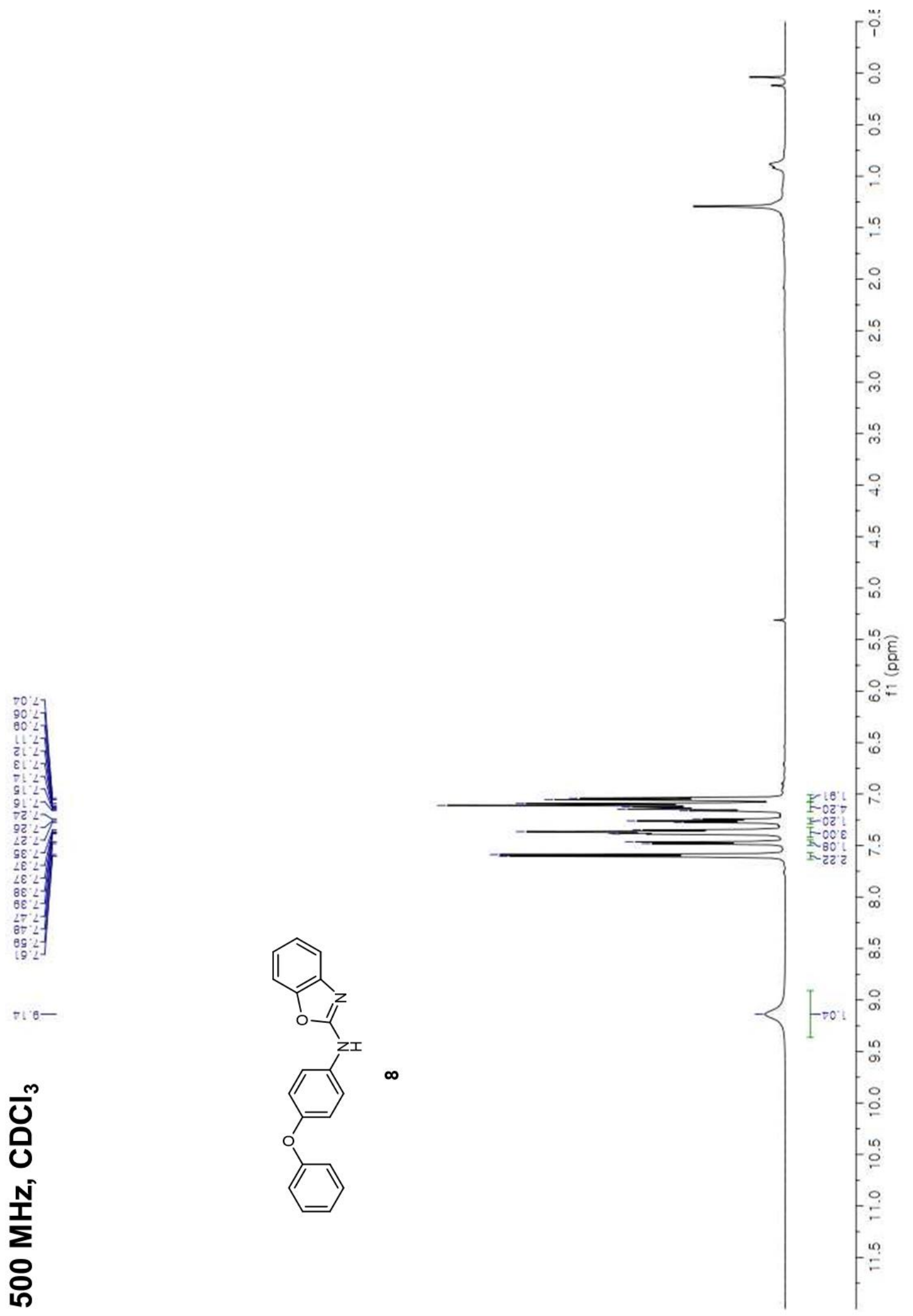

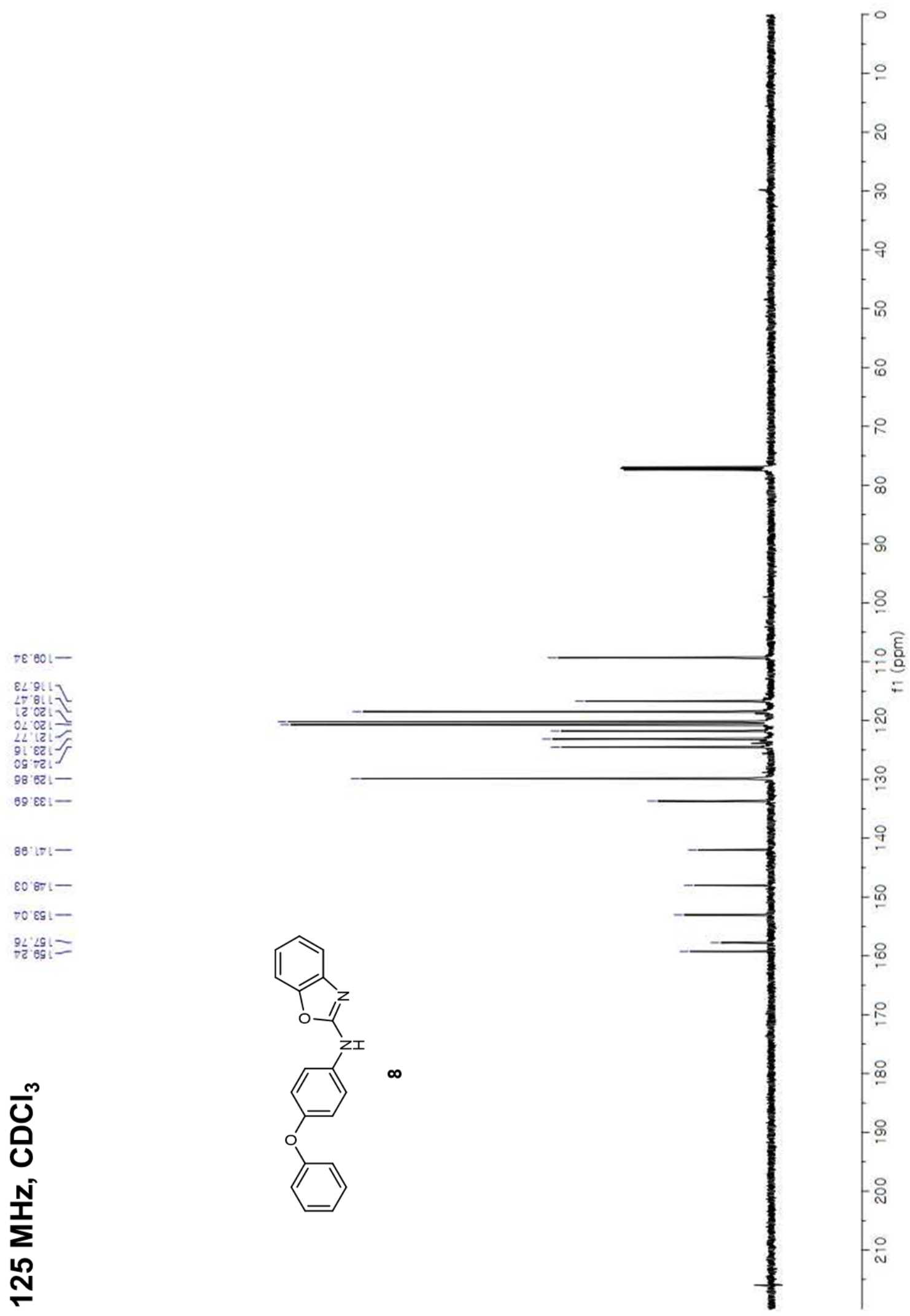


\section{U. References}

1. An, T.; Kang, B.; Kang, S.; Pac, J.; Youk, J.; Lin, D.; Lee, Y. Guanidine cyclic diimides and their polymers. Chem. Commun. 2019, 55, 10222-10225.

2. Zhao, H.; Hu, X.; Cao, K.; Zhang, Y.; Zhao, K.; Tang, C.; Feng, B. Synthesis and SAR of 4,5-dihydro$1 \mathrm{H}$-pyrazolo[4,3-h]quinazoline derivatives as potent and selective CDK4/6 inhibitors. Eur. J. Med. Chem. 2018, 157, 935-945.

3. Cocinero, E. J.; Stanca-Kaposta, E. C.; Gamblin, D. P.; Davis, B. G.; Simons, J. P. Peptide secondary structures in the gas phase: consensus motif of $N$-linked glycoproteins. J. Am. Chem. Soc. 2009, 131, 1282-1287.

4. M. J. Frisch, G. W. Trucks, H. B. Schlegel, G. E. Scuseria, M. A. Robb, J. R. Cheeseman, G. Scalmani, V. Barone, B. Mennucci, G. A. Petersson, H. Nakatsuji, M. Caricato, X. Li, H. P. Hratchian, A. F. Izmaylov, J. Bloino, G. Zheng, J. L. Sonnenberg, M. Hada, M. Ehara, K. Toyota, R. Fukuda, J. Hasegawa, M. Ishida, T. Nakajima, Y. Honda, O. Kitao, H. Nakai, T. Vreven, J. A., Jr. Montgomery, J. E. Peralta, F. Ogliaro, M. J. Bearpark, J. Heyd, E. N. Brothers, K. N. Kudin, V. N. Staroverov, R. Kobayashi, J. Normand, K. Raghavachari, A. P. Rendell, J. C. Burant, S. S. Iyengar, J. Tomasi, M. Cossi, N. Rega, N. J. Millam, M. Klene, J. E. Knox, J. B. Cross, V. Bakken, C. Adamo, J. Jaramillo, R. Gomperts, R. E. Stratmann, O. Yazyev, A. J. Austin, R. Cammi, C. Pomelli, J. W. Ochterski, R. L. Martin, K. Morokuma, V. G. Zakrzewski, G. A. Voth, P. Salvador, J. J. Dannenberg, S. Dapprich, A. D. Daniels, Ö. Farkas, J. B. Foresman, J. V. Ortiz, J. Cioslowski, D. J. Fox, Gaussian 09; Gaussian, Inc.: Wallingford, CT, 2009.

5. Bai, Y.; Shi, L.; Zheng, L.; Ning, S.; Che, X.; Zhang, Z.; Xiang, J. Electroselective and controlled reduction of cyclic imides to hydroxylactams and lactams. Org. Lett. 2021, 23, 2298-2302.

6. Wang, Y.; Cao, X.; Zhao, L.; Pi, C.; Ji, J.; Cui, X.; Wu, Y. Generalized chemoselective transfer hydrogenation/hydrodeuteration. Adv. Synth. Catal. 2020, 362, 4119-4129.

7. APEX3 and SADABS; Bruker AXS, Inc.: Madison, WI, 2016.

8. SHELXTL v 6.14; Bruker AXS, Inc.: Madison, WI, 2001.

9. Li, F.; Kang, Q.; Shan, H.; Chen, L.; Xie, J. Regioselective $\mathrm{N}$-alkylation of 2-aminoimidazoles with alcohols to 2-(N-alkylamino)imidazoles catalyzed by the $\left[\mathrm{Cp}^{*} \mid \mathrm{ICl}_{2}\right]_{2} / \mathrm{K}_{2} \mathrm{CO}_{3}$ system. Eur. J. Org. Chem. 2012, 5085-5092.

10. Tankam, T.; Srisa, J.; Sukwattanasinitt, M.; Wacharasindhu, S. Microwave-enhanced on-water amination of 2-mercaptobenzoxazoles to prepare 2-aminobenzoxazoles. J. Org. Chem. 2018, 83, 11936-11943.

11. Lee, Y.-W.; Luther, D. C.; Goswani, R.; Jeon, T.; Clark, V.; Elia, J.; Gopalakrishnan, S.; Rotello, V. M. Direct cytosolic delivery of proteins through coengineering of proteins and polymeric delivery vehicles. J. Am. Chem. Soc. 2020, 142, 4349-4355.

12. Wang, G.; Peng, Z.; Wang, J.; Li, J.; Li, X. Synthesis, biological evaluation and molecular docking study of $\mathrm{N}$-arylbenzo[d]oxazol-2-amines as potential $\alpha$-glucosidase inhibitors. Bioorg. Med. Chem. 2016, $24,5374-5379$. 